\title{
Navigation Using High-Frequency Ground Beacons and Ionosphere Model Corrections
}

\author{
A Dissertation \\ Presented to the Faculty of the Graduate School \\ of Cornell University \\ in Partial Fulfillment of the Requirements for the Degree of \\ Doctor of Philosophy
}

by

Yoav Baumgarten

December 2018 
(C) 2018 Yoav Baumgarten

ALL RIGHTS RESERVED 


\title{
NAVIGATION USING HIGH-FREQUENCY GROUND BEACONS AND IONOSPHERE MODEL CORRECTIONS
}

\author{
Yoav Baumgarten, Ph.D. \\ Cornell University 2018
}

This thesis defines and analyzes a navigation concept that relies on passive oneway ranging using pseudorange and beat carrier phase measurements of HighFrequency (HF) beacon signals that travel along non-line-of-sight paths via ionosphere refraction. The concept is being considered as a possible alternative to GNSS positioning and timing services.

The proposed system uses an array of ground stations that are placed at known, predetermined locations. HF signals are simultaneously transmitted from these ground-based beacons, and received at an unknown single receiver location. If the set of signals that reaches the user equipment receiver has sufficient geometric diversity, then the position and the clock offset of the receiver can be determined uniquely.

A significant challenge arises from ionospheric modeling uncertainties that cause errors in the determination of signal ray paths. Erroneous signal paths result in errors in the estimated user equipment position and clock offset. This challenge is addressed by estimating corrections to a parametric model of the ionosphere as part of the navigation solution. The coupled estimation problem is solved with a batch filter that 
simultaneously estimates the user equipment position, the clock offset, and corrections to an a priori ionosphere model.

The first part of this dissertation includes a theoretical background review, derivation of mathematical models, and descriptions of the structures of the developed batch filters. It considers two filter versions of this study that rely on two different physical models for the propagating HF signals.

The second part of the dissertation is dedicated to a system performance analysis and an assessment of algorithm functionality. This analysis is based on using data from a truth-model simulation. This is followed by a discussion that assesses performance sensitivities to setup characteristics.

A follow-on effort to this study is proposed, one in which algorithm functionality and performance would be examined with actual recorded data for input and signal processing. This proposed work is beyond the scope of this dissertation. 


\section{BIOGRAPHICAL SKETCH}

Yoav Baumgarten earned a Bachelor of Architecture (2002) and a Bachelor of Science in Aerospace Engineering (2008), both from the Technion - Israel Institute of Technology. He completed his Masters of Engineering degree in Aerospace Engineering from Cornell University in 2016 under Prof. Mark Psiaki. Yoav worked as a navigation engineer between 2006 and 2013 for Rafael Advanced Defense Systems, LTD. 


\section{ACKNOWLEDGEMENTS}

I would like to express my sincere gratitude to my advisor, Prof. Mark Psiaki, for his insightful, enriching and inspiring guidance. This has been a long, yet exceptionally rewarding journey.

I thank Prof. David Hysell for his significant contribution to this study.

Thank you Sharon for being by my side. 


\section{TABLE OF CONTENTS}

Biographical Sketch.....................................................................................ii

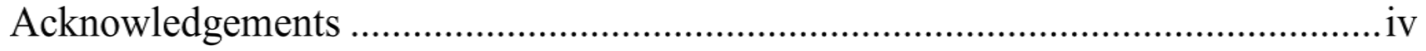

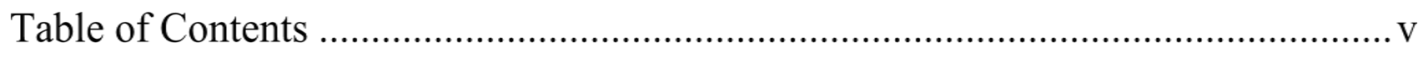

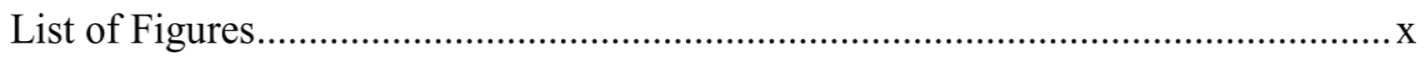

List of Tables .....................................................................................................

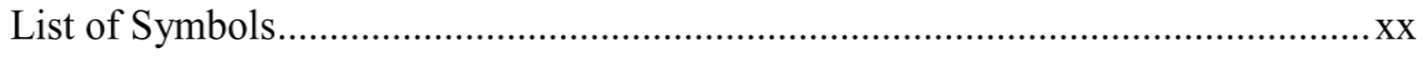

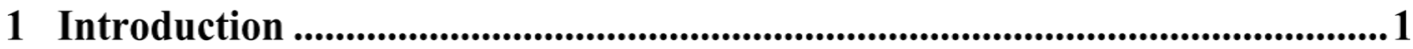

2 Physical Models ................................................................................................... 7

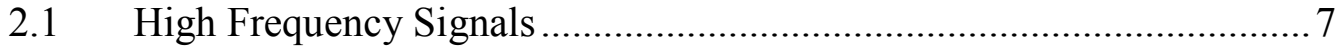

2.1.1 Signal structure and ranging accuracy .................................... 7

2.1.2 Physical characteristics of wave propagation in the ionosphere....... 8

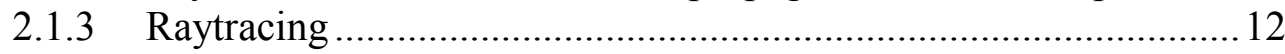

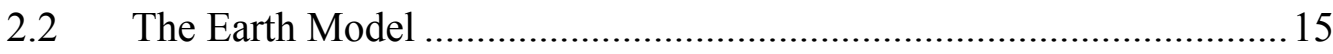

2.2.1 Earth geometry model ......................................................... 16

2.2.2 Earth magnetic field model .................................................... 17

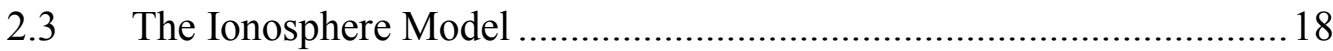

2.3.1 Ionosphere parameter errors and the $M$ covariance matrix............21

2.3.2 The Ionosphere Errors Index.................................................... 23

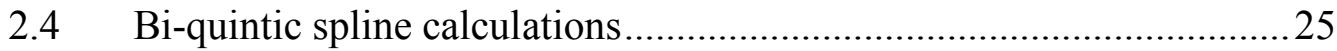

3 Bounce Points, Ray-Hops, and Ray-Paths .....................................................28

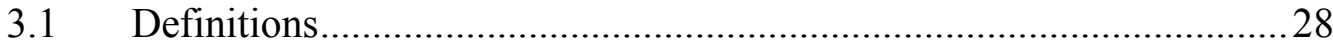

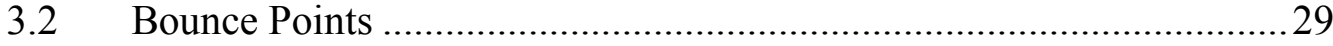

3.2.1 Bounce point properties and representation...............................29

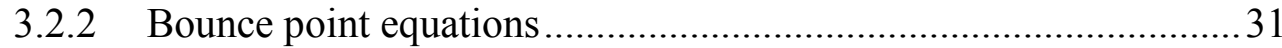

Single-Hop Calculations .......................................................... 33

3.3.1 The fundamental nonlinear two point boundary value problem .....33 


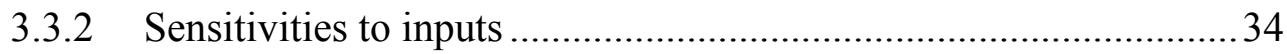

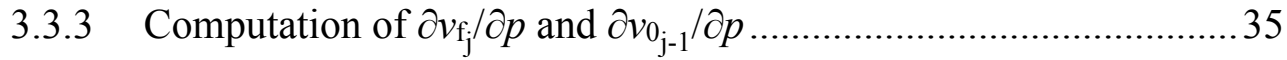

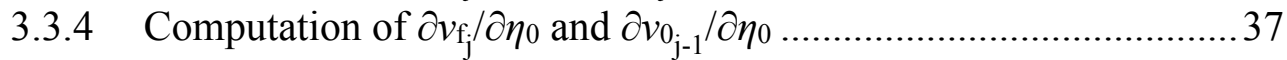

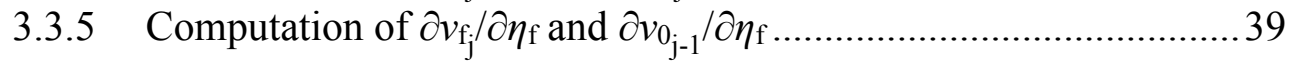

3.4 Multiple-Hop Calculations: The Ray-Path Solver ..................................41

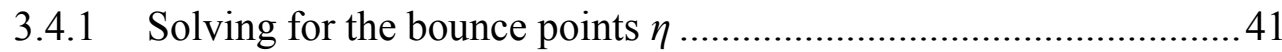

3.4.2 Ray-path solver robustness ........................................................... 44

3.4.3 Ray-paths' setup feasibility, solution existence and uniqueness ....45

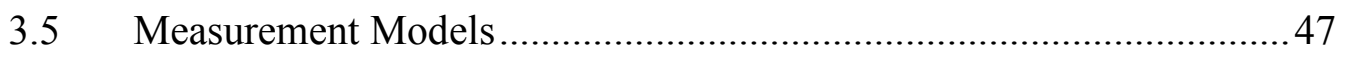

3.5.1 The group delay measurement model ……….............................. 47

3.5.2 The range-equivalent beat carrier phase model ...............................49

3.5.3 Measurement model sensitivity matrices ..........................................53

\section{Batch Estimation of Receiver Position, Receiver Clock Offset, and} Ionosphere Parameters......................................................................56

4.1 Batch Filter Problem and Solution...................................................56

4.1.1 The nominal case .....................................................................56

4.1.2 The case of a known receiver position and clock error.....................57

4.2 A Modified Gauss-Newton Method.......................................................58

4.2.1 The top-level procedure ………………………...........................5

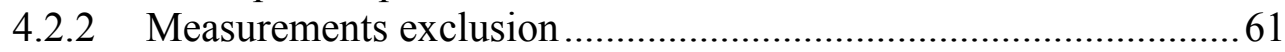

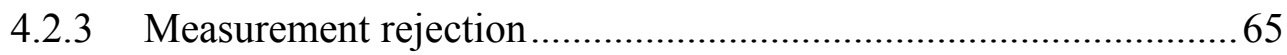

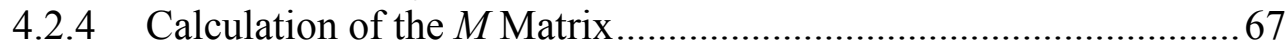

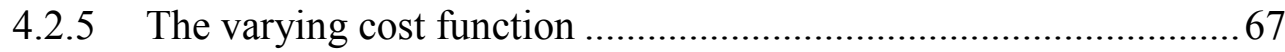

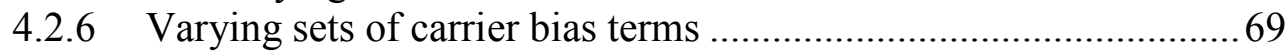

4.2.7 Iterating when position solution is far from convergence.................69

4.3 Predicted Estimation Errors ................................................................

4.4 The Mean Square Error Matrix and the A Posteriori Covariance .........73

4.4.1 The case of random ionosphere parameters errors..........................73

4.4.2 The case of constant a priori ionosphere parameters errors ............74

4.4.3 Ionosphere model covariance mismatch ..........................................74

4.4.4 Measurement noise covariance mismatch......................................75

4.4.5 The horizontal and vertical position error ellipses...........................76

5 A Simplified Model-Based Estimator ................................................................78

5.1 Physical and Mathematical Models ......................................................79

5.1.1 Earth and the ionosphere...........................................................79

5.1.2 Bounce points, ray-paths, and the measurement model.................... 80

5.1.3 Bounce point equations ................................................................... 81

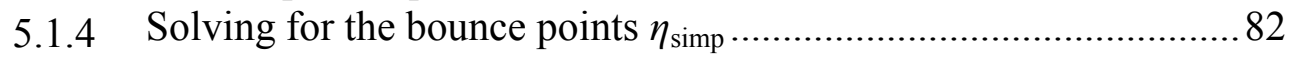


5.2 A Batch Filter for the Simplified Model ............................................... 84

5.2.1 The Modified Gauss-Newton Method ............................................. 84

5.2.2 Measurement Model Sensitivity Matrices ......................................... 85

5.2.3 Calculation of Jacobian matrices .................................................... 86

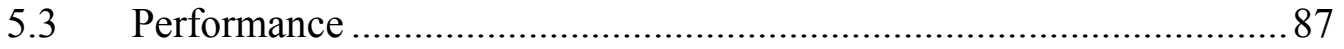

6 Algorithm Operation and Validation ....................................................................88

6.1 Truth-Model Simulation and Batch Filter Monitoring .......................... 88

6.1.1 Execution modes ....................................................................92

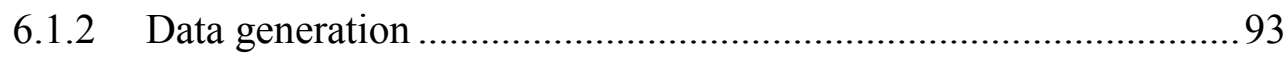

6.2 Normal Batch Filtering Algorithm Operation and Functionality..........95

6.2.1 Ray-path solving attempts........................................................95

6.2.2 Cost function evolution................................................................ 100

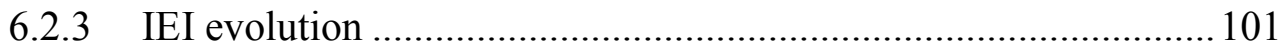

6.3 Process failure patterns and solution divergence ................................. 102

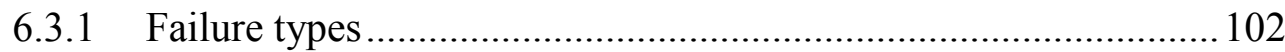

6.3.2 Causes of algorithm failure to converge and known algorithm

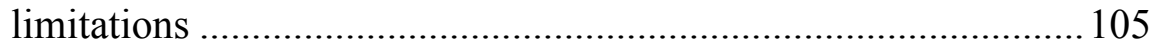

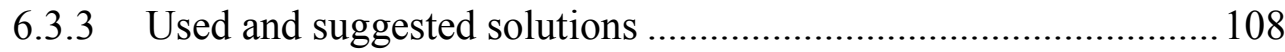

6.4 Solution Convergence to the Problem's Optimal Solution.................... 108

6.4.1 Cost function characterization through 3D mapping .....................109

6.4.2 Convergence validation ..........................................................111

6.4.3 Rate of convergence ..................................................................112

6.5 Covariance Analysis Validation .......................................................... 113

6.5.1 The case of random ionosphere parameters errors.........................113

6.5.2 The case of constant a priori ionosphere parameter errors............116

7 Results For Batch-Filtering Test Cases .................................................................120

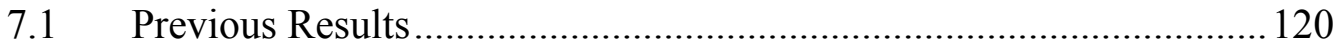

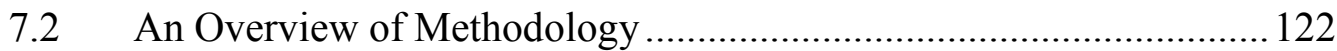

7.2.1 Simulation-based analysis............................................................122

7.2.2 The statistical analysis for a random-ionosphere model................123

7.2.3 Test case groups and test case classes............................................126

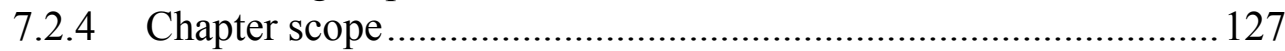

7.3 Test Cases Using Group Delay Only, Fixed Ionosphere Model

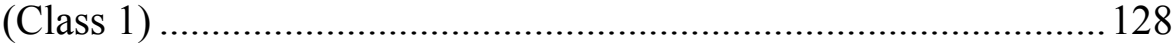

7.3.1 Test cases with a medium number of available ray-paths (Group B) ........................................................................... 130

7.3.2 Test cases with a low number of available ray-paths (Group A)..150 
7.3.3 Test cases with a high number of available ray-paths (Group C). 151

7.3.4 Test cases using only group delay, summary..................................155

7.4 Test Cases Using Combined Code and Carrier Phase Ranging,

Fixed Ionosphere Model (Class 2)

7.4.1 Test cases with a high number of available ray-paths (Group E) .159

7.4.2 Test cases with a medium number of available ray-paths

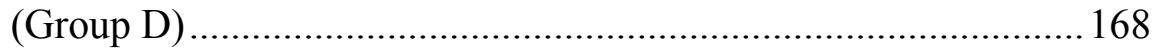

7.4.3 Summary of test cases using combined code and carrier ranging

7.5 Test Cases Using Group Delay Only, Random Ionosphere Model (Class 3)

7.5.1 Test cases with a high number of available ray-paths (Group F) 174

7.6 Test Cases Using Combined Code and Carrier Phase Ranging, Random Ionosphere Model (Class 4)

7.6.1 Test cases with a high number of available ray-paths (Group H). 181

7.6.2 Test cases with a medium number of available ray-paths (Group G)

8 System Performance Analysis, Discussion, and Future Directions 200

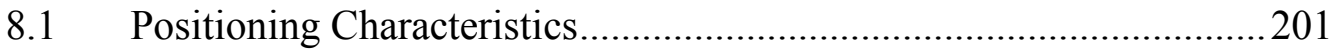

8.1.1 Sensitivity to the available number of measurements....................203

8.1.2 Sensitivity to signals' vertical direction of arrival .........................204

8.1.3 Sensitivity to a priori ionosphere model error...............................205

8.1.4 Sensitivity to ground stations placement .......................................207

8.1.5 Sensitivity to measurement noise covariance matrix and actual

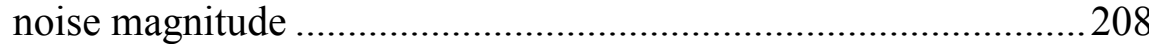

8.1.6 Sensitivity to additional parameters ..............................................210

8.2 A Posteriori Ionosphere Model Accuracy ..........................................211

8.2.1 Scenarios with a High Number of Available Signals ....................211

8.2.2 Scenarios with Medium and Low Numbers of Available

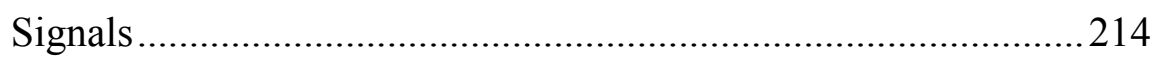

8.3 Batch-Filtering Algorithm Functionality ..........................................216

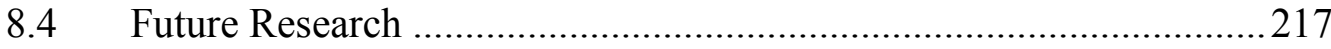

9 Summary and Conclusion.................................................................................220

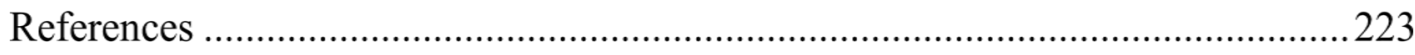

APPENDIX A Auxiliary Calculations For Physical and Mathemathical

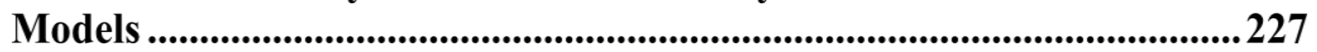

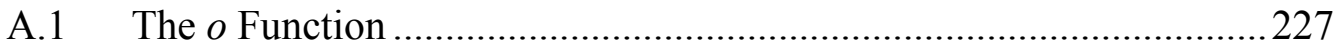


APPENDIX B Technical Notes for the Simplified Ray-Path Model Filter...229

B.1 Computing Sensitivity of Bounce-Points Equations to Bounce-

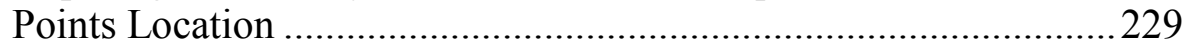

B.2 Setting up the $\partial g / \partial \eta$ Sensitivity Matrix .............................................231

APPENDIX C Implementation and Practicalities..................................................234

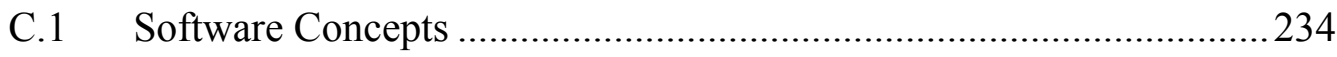

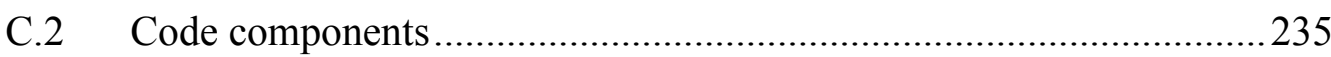

C.3 Hardware and Process Runtime ...........................................................2236 


\section{LIST OF FIGURES}

Figure 1: The 424 grid nodes placement map for an example latitude/longitude bi-quintic spline.

Figure 2: IEI values vs. time difference (top); IEI histogram (bottom). 24

Figure 3: The bi-quintic spline calculation latitude/longitude grid example. 25

Figure 4: The ray-path definitions and notation. 29

Figure 5: An example of the time-dependent signal frequency and the corresponding phase. The nominal transmitted frequency is shown in blue in the left-hand panel. The received frequency is shown as the dashed green curve in the same plot. The corresponding phases are shown in the right-hand panel.

Figure 6: Illustration of: the NE or EU reference frame, the $f$ reference frame, the $90 \%$ error ellipse and its two axes.

Figure 7: A typical test case setup used with the truth-model simulation.

Figure 8: An example process monitoring screen for test case E0 in Nominal Scenario mode.

Figure 9: Proper algorithm functionality is evident in successfully computed ray-paths (not all presented) that have the same characteristics as their true ray-path equivalents. This figure plots true (thick lines) versus estimated (thin lines) ray-paths for an early iteration of the Gauss-Newton process for Test Case 3411.

Figure 10: Process monitoring screen for Test Case 3811 in Nominal Scenario mode, demonstrating occasional failures in attempts to solve for rayhops and ray-paths during execution of the batch filter.

Figure 11: Process monitoring screen for Test Case 3531 in Nominal Scenario mode, demonstrating massive ray-paths solver failures at the early stages of the iterative batch filtering process due to a very poor $a$ priori ionosphere model. Both final position error and final IEI $\xi$ are small. 
Figure 12: Process monitoring screen (partial view) for Test Case 3431 in Nominal Scenario mode. Note the behavior of the IEI parameter $\xi$.

Figure 13: Process monitoring screen for run 0002 of a Monte Carlo Analysis conducted for Test Case 3411A. With more than 100 process steps, the algorithm failed to drive the unknown position solution closer to its optimal value, yet $\xi$ values remain fairly steady.

Figure 14: A partial view of the process monitoring screen for a Nominal Scenario simulation of Test Case 3522. Magnitude of errors for all unknowns indicates process divergence.

Figure 15: Process monitoring screen for run 0087 of a Monte Carlo Analysis conducted for Test Case 3911. Estimates remain close to their optimal values, yet the algorithm fails to bring them to convergence.... 105

Figure 16: True (blue) and computed (green) trajectories for ray-path 33 of run 0087 of a Monte Carlo analysis for Test Case 3911. Top: the full ray-paths; Bottom: a closer look near the true (blue) and estimated (green) trajectory end points

Figure 17: The true trajectory for ray-path 9 (blue) and the estimated trajectory (green) for that ray path, calculated for Monte Carlo run 0002 of Test Case 3411A. Inconsistency in signal direction of arrival yields significant, unaccounted for measurement errors.

Figure 18: A spatial cost function map for a 12-ray-paths test case. Cost function values, shown by color coding, have been computed in the vicinity of the optimal solution for the receiver location.

Figure 19: Monte Carlo and theoretical position error distributions for Test Case 5511.17 out of 200 points fell outside the horizontal $90 \%$ error ellipse and 22 points fall outside the vertical $90 \%$ error ellipse.

Figure 20: Monte Carlo and theoretical position error distributions for Test Case 5611. Twenty two dots out of 200 fall outside the horizontal $90 \%$ error ellipse, and 21, dots fall outside the vertical Up-East $90 \%$ error ellipse.

Figure 21: Empirical and theoretical position error distributions for Test Case 3511. This plot demonstrates mean error and error covariance consistency for the important case of constant a priori errors in the parametric ionosphere model. 
Figure 22: Empirical and theoretical position error distributions for Test Case 3531. Agreement between the two analyses is demonstrated for this setup that is characterized with a very significant error in the a priori parameterized ionosphere model. In this plot, the $90 \%$ error ellipses are centered about the theoretical mean.

Figure 23: Empirical and theoretical position error distributions for Test Case 3611B for which much larger measurement errors were assumed. With 18 of 200 points falling outside the horizontal error ellipse and 16 out of 200 points falling outside the vertical error ellipse, consistency is demonstrated for this test case.

Figure 24: Setup for test cases of Group B, ground stations (blue circles), true ray-path trajectories (green curves), and position solution convergence trajectory (red curve) for Test Case B0.

Figure 25: A priori (top) and a posteriori (bottom) errors for the ionospheric peak electron density height parameter $h_{\max }$ for a Nominal Scenario Mode run of Test Case B0.

Figure 26: A priori (top) and a posteriori (bottom) errors for the ionospheric scale height parameter $h_{\mathrm{sf}}$ for a Nominal Scenario Mode run of Test Case B0.

Figure 27: A priori (top) and a posteriori (bottom) errors for the ionospheric vertical total electron content parameter VTEC for a Nominal Scenario Mode run of Test Case B0.

Figure 28: Position error pattern for Test Case B0; Monte Carlo analysis scatter plot (blue); Horizontal 90\% errors ellipse (top plot, magenta); Vertical 90\% errors ellipse (bottom plot, green).

Figure 29: The statistical characteristics of the a posteriori ionosphere model errors for Test Case B0. The top three panels show histograms of the $80^{\text {th }}$ and $95^{\text {th }}$ CONUS area percentile error limits for the three Chapman parameters. The bottom panel is a histogram of $\xi$ values. Dashed lines mark the $80^{\text {th }}$ and $95^{\text {th }}$ percentile values of these quantities for the a priori ionosphere parameter error vector, except in the bottom panel the dashed line marks the a priori error's mean..... 137

Figure 30: Position error pattern for Test Case B1; Monte Carlo analysis scatter plot (blue); Horizontal 90\% errors ellipse (top plot, magenta); Vertical 90\% errors ellipse (bottom plot, green) 
Figure 31: The statistical characteristics of the a posteriori ionosphere model errors for Test Case B1. The top three panels contain histograms of the $80^{\text {th }}$ and $95^{\text {th }}$ percentile error values for the three Chapman parameters. The bottom panel is a histogram of $\xi$ values. Dashed lines mark the $80^{\text {th }}$ and $95^{\text {th }}$ percentile values of these quantities for the a priori ionosphere parameter error vector, except in the bottom panel the dashed line marks the a priori error's mean.

Figure 32: A priori (top) and a posteriori (bottom) errors for the ionospheric peak electron density height parameter $h_{\max }$ for Test Case B2.

Figure 33: A priori (top) and a posteriori (bottom) errors for the ionospheric scale height parameter $h_{\text {sf }}$ for Test Case B2

Figure 34: Position error pattern for Test Case B2; Monte Carlo analysis scatter plot (blue); Horizontal 90\% errors ellipse (top plot, magenta); Vertical $90 \%$ errors ellipse (bottom plot, green); The dashed gray ellipses plot the $90 \%$ errors ellipses for base Test Case B0 and are shown here for comparison purposes.

Figure 35: The statistical characteristics of the a posteriori ionosphere model errors for Test Case B2. The top three panels contain histograms of the $80^{\text {th }}$ and $95^{\text {th }}$ percentile error values for the three Chapman parameters. The bottom panel is a histogram of $\xi$ values. Dashed lines mark the $80^{\text {th }}$ and $95^{\text {th }}$ percentile values of these quantities for the a priori ionosphere parameter error vector, except in the bottom panel the dashed line marks the a priori error's mean.

Figure 36: Setup for Test Case B3 with ground stations (blue circles) placed along the coastal and land borders of the US, true ray-paths trajectories (green), and convergence of position solution (red).

Figure 37: A priori (top) and a posteriori (bottom) errors for the ionospheric peak electron density height parameter $h_{\max }$ for a Nominal Scenario Mode run of Test Case B3.

Figure 38: A priori (top) and a posteriori (bottom) errors for the ionospheric vertical total electron content parameter VTEC for a Nominal Scenario Mode run of Test Case B3.

Figure 39: Position error pattern for Test Case B3; Monte Carlo analysis scatter plot (blue); Horizontal 90\% errors ellipse (top plot, magenta); Vertical 90\% errors ellipse (bottom plot, green); The dashed gray ellipses plot the $90 \%$ error ellipses for base Test Case B0 and are shown here for comparison purposes. 
Figure 40: Position error pattern for Test Case B4; Monte Carlo analysis scatter plot (blue); Horizontal 90\% errors ellipse (top plot, magenta); Vertical 90\% errors ellipse (bottom plot, green).

Figure 41: Position error pattern for Test Case A0; Monte Carlo analysis scatter plot (blue); Horizontal 90\% error ellipse (top plot, magenta); Vertical 90\% error ellipse (bottom plot, green). The dashed gray ellipses plot the $90 \%$ error ellipses for base Test Case B0 and are shown here for comparison purposes.

Figure 42: Setup for test cases of Groups C and E, transmitters (blue circles), true ray-path trajectories (green curves), and convergence of position solution for Test Case C0 (red curve).

Figure 43: Position error pattern for Test Case C0; Monte Carlo analysis scatter plot (blue); Horizontal 90\% errors ellipse (top plot, magenta); Vertical $90 \%$ errors ellipse (bottom plot, green); The dashed gray ellipses are the $90 \%$ error ellipses for Test Case B0, which are shown here for comparison purposes.

Figure 44: Position error pattern for Test Case C4; Monte Carlo analysis scatter plot (blue); Horizontal 90\% errors ellipse (top plot, magenta); Vertical 90\% errors ellipse (bottom plot, green).

Figure 45: Position error pattern for Test Case E0; Monte Carlo analysis scatter plot (blue); Horizontal 90\% errors ellipse (top plot, magenta); Vertical 90\% errors ellipse (bottom plot, green).

Figure 46: The statistical characteristics of the a posteriori ionosphere model errors for Test Case E0. The top three panels contain histograms of the $80^{\text {th }}$ and $95^{\text {th }}$ CONUS area percentile error values for the three Chapman parameters. The bottom panel is a histogram of $\xi$ values. Dashed lines mark the $80^{\text {th }}$ and $95^{\text {th }}$ percentile values of these quantities for the a priori ionosphere parameter error vector, except in the bottom panel the dashed line marks the a priori error's mean...... 161

Figure 47: A priori (top) and a posteriori (bottom) errors for the ionospheric peak electron density height $h_{\mathrm{sf}}$ parameter for Test Case E0.

Figure 48: Position error pattern for Test Case E1; Monte Carlo analysis scatter plot (blue); Horizontal 90\% errors ellipse (top plot, magenta); Vertical $90 \%$ errors ellipse (bottom plot, green); The dashed gray ellipses are the $90 \%$ errors ellipses for base Test Case E0, shown here for purposes of comparison. 
Figure 49: A priori (top) and a posteriori (bottom) errors for the ionospheric vertical total electron content parameter VTEC for a Nominal Scenario Mode run of Test Case E2......

Figure 50: Position error pattern for Test Case E2; Monte Carlo analysis scatter plot (blue); Horizontal 90\% errors ellipse (top plot, magenta); Vertical 90\% errors ellipse (bottom plot, green); The dashed gray ellipses are the $90 \%$ error ellipses for base Test Case E0 that are shown here for comparison purposes.

Figure 51: Position error pattern for Test Case E3; Monte Carlo analysis scatter plot (blue); Horizontal 90\% errors ellipse (top plot, magenta); Vertical 90\% errors ellipse (bottom plot, green); The dashed gray ellipses are the $90 \%$ error ellipses for base Test Case E0, shown here for comparison purposes.

Figure 52: A priori (top) and a posteriori (bottom) errors for the ionospheric scale height $h_{\text {sf }}$ parameter for a Nominal Scenario Mode run of Test Case E3.

Figure 53: Position error pattern for Test Case E4; Monte Carlo analysis scatter plot (blue); Horizontal 90\% errors ellipse (top plot, magenta); Vertical 90\% errors ellipse (bottom plot, green); The dashed gray ellipses are the $90 \%$ error ellipses for base Test Case E0, shown here for comparison purposes.

Figure 54: Position error pattern for Test Case D0; Monte Carlo analysis scatter plot (blue); Horizontal 90\% errors ellipse (top plot, magenta); Vertical 90\% errors ellipse (bottom plot, green); The dashed gray ellipses are the $90 \%$ error ellipse for the base test case of Group E (Test Case E0), shown here for comparison purposes.

Figure 55: The statistical characteristics of the a posteriori ionosphere model errors for Test Case D0, including histograms of the $80^{\text {th }}$ and $95^{\text {th }}$ CONUS area percentile error values for the three Chapman parameters. The bottom panel is a histogram of $\xi$ values. Dashed lines mark the $80^{\text {th }}$ and $95^{\text {th }}$ percentile values of these quantities for the a priori ionosphere parameter error vector, except in the bottom panel the dashed line marks the $a$ priori error's mean.

Figure 56: Position error pattern for Test Case D2; Monte Carlo analysis scatter plot (blue); Horizontal 90\% errors ellipse (top plot, magenta); Vertical 90\% errors ellipse (bottom plot, green); The dashed gray ellipses are the $90 \%$ error ellipse for base Test Case D0 and are included here for comparison purposes. 
Figure 57: Horizontal and vertical 90\% error ellipses for Test Case F0 with different values of $\gamma$.

Figure 58: Eightieth percentile value maps for the $a$ priori (left column) and $a$ posteriori (right column) estimates' errors for the ionospheric peak electron density height parameter $h_{\max }$ for Test Case F0. Each row corresponds to a different value of $\gamma$.

Figure 59: Eightieth percentile value maps for the a priori (left column) and $a$ posteriori (right column) estimates' errors for the ionospheric scale height parameter $h_{\mathrm{sf}}$ for Test Case F0. Each row corresponds to a different value of $\gamma$.

Figure 60: Eightieth percentile value maps for the a priori (left column) and $a$ posteriori (right column) estimates' errors for the ionospheric vertical total electron content parameter $V T E C$ for Test Case F0. Each row corresponds to a different value of $\gamma$

Figure 61: Horizontal and vertical 90\% error ellipses for Test Case H0 with different values of $\gamma$

Figure 62: Eightieth percentile value maps for the a priori (left column) and $a$ posteriori (right column) estimates' errors for the ionospheric peak electron density height parameter $h_{\max }$ for Test Case H0. Each row corresponds to a different value of $\gamma$.

Figure 63: Eightieth percentile value maps for the a priori (left column) and $a$ posteriori (right column) estimates' errors for the ionospheric scale height parameter $h_{\mathrm{sf}}$ for Test Case H0. Each row corresponds to a different value of $\gamma$.

Figure 64: Eightieth percentile value maps for the a priori (left column) and $a$ posteriori (right column) estimates' errors for the ionospheric vertical total electron content parameter VTEC for Test Case H0. Each row corresponds to a different value of $\gamma$

Figure 65: Horizontal and vertical 90\% error ellipses for Test Case H1 with different values of $\gamma$. The dashed ellipses in gray are the $90 \%$ error ellipses for Test Case $\mathrm{H} 0$ with $\gamma=1$.

Figure 66: Eightieth percentile value maps for the a priori (left column) and $a$ posteriori (right column) estimates' errors for the ionospheric peak electron density height parameter $h_{\max }$ for Test Case H1. Each row corresponds to a different value of $\gamma$. 
Figure 67: Eightieth percentile value maps for the a priori (left column) and $a$ posteriori (right column) estimates' errors for the ionospheric scale height parameter $h_{\mathrm{sf}}$ for Test Case H1. Each row corresponds to a different value of $\gamma$.

Figure 68: Eightieth percentile value maps for the a priori (left column) and $a$ posteriori (right column) estimates' errors for the ionospheric vertical total electron content parameter VTEC for Test Case H1. Each row corresponds to a different value of $\gamma$.

Figure 69: Horizontal and vertical 90\% error ellipses for Test Case $\mathrm{H} 3$ with different values of $\gamma$. The dashed ellipses in gray are the $90 \%$ error ellipses for Test Case $\mathrm{H} 0$ with $\gamma=1$.

Figure 70: Eightieth percentile value maps for the a priori (left column) and $a$ posteriori (right column) estimates' errors for the ionospheric peak electron density height parameter $h_{\max }$ for Test Case H3. Each row corresponds to a different value of $\gamma$.

Figure 71: Eightieth percentile value maps for the a priori (left column) and $a$ posteriori (right column) estimates' errors for the ionospheric scale height parameter $h_{\text {sf }}$ for Test Case H3. Each row corresponds to a different value of $\gamma$.

Figure 72: Eightieth percentile value maps for the a priori (left column) and $a$ posteriori (right column) estimates' errors for the ionospheric vertical total electron content parameter VTEC for Test Case H3. Each row corresponds to a different value of $\gamma$.

Figure 73: Horizontal and vertical 90\% error ellipses for Test Case $\mathrm{H} 4$ with different values of $\gamma$. The dashed ellipses in gray are the $90 \%$ error ellipses for Test Case $\mathrm{H} 0$ with $\gamma=1$.

Figure 74: Horizontal and vertical $90 \%$ error ellipses for Test Case G0 with different values of $\gamma$. The dashed ellipses in gray are the $90 \%$ error ellipses for Test Case H0 with $\gamma=1$. ....

Figure 75: Eightieth percentile value maps for the a priori (left column) and $a$ posteriori (right column) estimates' errors for the ionospheric peak electron density height parameter $h_{\max }$ for Test Case G0. Each row corresponds to a different value of $\gamma$.

Figure 76: Eightieth percentile value maps for the a priori (left column) and $a$ posteriori (right column) estimates' errors for the ionospheric scale height parameter $h_{\text {sf }}$ for Test Case G0. Each row corresponds to a different value of $\gamma$. 
Figure 77: Eightieth percentile value maps for the $a$ priori (left column) and $a$ posteriori (right column) estimates' errors for the ionospheric vertical total electron content parameter VTEC for Test Case G0. Each row corresponds to a different value of $\gamma$....................................... 199

Figure 78: The form of a 4 bounce-points ray-path's Jacobian sensitivity matrix. 


\section{LIST OF TABLES}

Table 1: Setup configuration for Class 1's three groups and eight test cases ......... 128

Table 2: Class 1 test cases' position estimation error characteristics...................... 155

Table 3: Class 1 test cases' ionosphere model estimation error characteristics...... 156

Table 4: Setup configuration for two groups and seven test cases of Class 2........ 158

Table 5: Class 2 test cases' primary position error characteristics....................... 172

Table 6: Class 2 test cases' primary ionosphere model errors characteristics......... 173

Table 7: Setup configuration for two groups and five test cases of Class $4 \ldots \ldots \ldots \ldots . . .180$ 


\section{LIST OF SYMBOLS}

\begin{tabular}{|c|c|c|}
\hline Symbol & Meaning & $\begin{array}{l}\text { Introduced in } \\
\text { chapter/section }\end{array}$ \\
\hline$A_{0}$ & signal amplitude & 2.1 .2 \\
\hline$\underline{B}$ & magnetic field vector & 2.1 .2 \\
\hline$\hat{B}_{0}$ & $\begin{array}{l}\text { a unit vector that points in the direction of the ambient geomagnetic } \\
\text { filed vector }\end{array}$ & 2.1 .3 \\
\hline $\mathrm{c}$ & speed of light & 2.1 .2 \\
\hline$D$ & total derivative operator & 3.4 .1 \\
\hline$\hat{D}$ & $3 \times 3$ matrix used with Hamiltonian formulation & 2.1 .3 \\
\hline$d x$ & norm of a Newton step correction vector & 6.3 .1 \\
\hline$\underline{E}$ & electric field vector & 2.1 .2 \\
\hline$F$ & an auxiliary reference frame & 4.4 .5 \\
\hline$f$ & function vector & 2.1 .3 \\
\hline$f_{s}$ & signal frequency & 3.5 .2 \\
\hline$g$ & bounce points equations function & 3.2 .1 \\
\hline$\tilde{g}$ & bounce points equations function, alternative formulation & 3.2 .1 \\
\hline$g_{\text {simp }}$ & bounce points equations, simplified model & 5.1 .2 \\
\hline$\tilde{g}_{\text {simp }}$ & bounce points equations, simplified model, alternative formulation & 5.2 .2 \\
\hline$H$ & Hamiltonian & 2.1 .3 \\
\hline halt & altitude above the WGS-84 ellipsoid & 2.2 .1 \\
\hline$h_{\mathrm{c}}$ & range-equivalent carrier phase change measurement & 3.5 .2 \\
\hline$\breve{h_{\mathrm{c}}}$ & $\begin{array}{l}\text { range-equivalent carrier phase change measurement, alternative } \\
\text { form }\end{array}$ & 3.5 .2 \\
\hline$\tilde{h_{\mathrm{c}}}$ & $\begin{array}{l}\text { range-equivalent carrier phase change measurement, alternative } \\
\text { form }\end{array}$ & 3.5 .2 \\
\hline$h_{\mathrm{DTM}}$ & Earth surface's height above the WGS-84 ellipsoid & 3.2 .2 \\
\hline$h_{\mathrm{g}}$ & group delay measurement & 3.5 .1 \\
\hline$\breve{h_{\mathrm{g}}}$ & group delay measurement, alternative form & 3.5 .1 \\
\hline$\tilde{h_{\mathrm{g}}}$ & group delay measurement, alternative form & 3.5 .1 \\
\hline$h_{\max }$ & $\begin{array}{l}\text { Chapman profile's parameter of altitude of maximum electron } \\
\text { density }\end{array}$ & 2.3 \\
\hline$h_{s f}$ & Chapman profile's parameter of altitude scale height & 2.3 \\
\hline$\tilde{h}_{\text {simp }}$ & group delay measurement, simplified model & 5.1 .2 \\
\hline$J_{1}$ & cost function for the top-level Gauss-Newton method & 4.1 .1 \\
\hline$J_{1, \text { simp }}$ & $\begin{array}{l}\text { cost function for the top-level Gauss-Newton method, simplified } \\
\text { model }\end{array}$ & 5.2 \\
\hline
\end{tabular}




\begin{tabular}{|c|c|c|}
\hline$J_{2}$ & cost function for the top-level Gauss-Newton method & 4.2 .7 \\
\hline$J_{4}$ & cost function for the top-level Gauss-Newton method & 4.1.2 \\
\hline$J_{\text {reeval }}$ & reevaluated cost function & 4.2 .2 \\
\hline$J_{\mathrm{RP}}$ & the ray-path solver's cost function & 3.4 .1 \\
\hline$J_{\mathrm{RP}, \text { simp }}$ & the ray-path solver's cost function, simplified model & 5.1 .4 \\
\hline$K$ & coefficients matrix & 2.1 .2 \\
\hline$k$ & wave number or bounce point index, depending on the context & 2.1 .2 \\
\hline$\underline{k}$ & wave vector & 2.1 .2 \\
\hline$k_{0}$ & free-space wave number & 2.1 .3 \\
\hline$k_{\mathrm{x}}, k_{\mathrm{y}}, k_{\mathrm{z}}$ & Cartesian components of the signal wave vector $\underline{k}$ & 2.1 .3 \\
\hline$M$ & ionospheric parameters' a priori covariance matrix & 4.1 .1 \\
\hline$M_{0}$ & ionospheric parameters' a priori covariance matrix & 2.3 .1 \\
\hline$M_{\mathrm{c}}$ & considered ionosphere parameters covariance matrix & 4.4 .3 \\
\hline$m$ & number of bounce points & 3.4 .1 \\
\hline$m_{e}$ & electron mass & 2.1 .2 \\
\hline$N$ & number of measurements & 3.5 .1 \\
\hline$N_{e}$ & electron density & Chapter 1 \\
\hline$n$ & Index of refraction & 2.1 .2 \\
\hline$n_{\mathrm{AH}}$ & lossy Appleton-Hartree index of refraction & 2.1 .3 \\
\hline$n_{\text {simp }}$ & index of refraction, simplified model & 5.1 .1 \\
\hline$P$ & range-equivalent beat carrier phase & 2.1 .3 \\
\hline$P^{\prime}$ & range-equivalent group delay & 2.1 .3 \\
\hline$\underline{p}$ & $\begin{array}{l}\text { a set of parameters that characterizes the ionosphere electron } \\
\text { density profile }\end{array}$ & 2.1 .3 \\
\hline$p_{\mathrm{a}}$ & $\begin{array}{l}\text { a set of parameters that characterizes the value and derivative of a } \\
\text { single Chapman parameter }\end{array}$ & 2.3 \\
\hline$\hat{\underline{p}}$ & $\begin{array}{l}\text { a set of parameters that characterizes the ionosphere electron } \\
\text { density profile near all considered ray-paths }\end{array}$ & 3.5 .1 \\
\hline$\hat{p}_{\mathrm{j}}$ & $\begin{array}{l}\text { a set of parameters that characterizes the ionosphere electron } \\
\text { density profile in the vicinity of the } j^{\text {th }} \text { ray-path }\end{array}$ & 3.1 \\
\hline$\tilde{\underline{q}}$ & $\begin{array}{l}\text { a set of parameters that characterizes the ionosphere electron } \\
\text { density profile in the vicinity of a ray-hop }\end{array}$ & 3.3 \\
\hline$q_{e}$ & electron charge & 2.1 .2 \\
\hline$R$ & measurement error covariance matrix & 4.1 .1 \\
\hline$R_{\mathrm{c}}$ & Earth's equatorial radius & 2.2 .1 \\
\hline$R_{\mathrm{g}}$ & group delay measurement noise covariance matrix & 3.5 .1 \\
\hline$R_{\mathrm{p}}$ & Earth's polar radius & 2.2 .1 \\
\hline$\underline{r}$ & location given in Cartesian coordinates & Chapter 1 \\
\hline$r_{1}, r_{2}, r_{3}$ & general Cartesian coordinates & 2.2 .1 \\
\hline$\underline{r}_{\mathrm{R}}$ & location of the receiver & 3.1 \\
\hline$\underline{r}_{\mathrm{w}}$ & wave front location & 2.1 .3 \\
\hline$s$ & ray hop & 3.1 \\
\hline$t$ & time & 2.2 .1 \\
\hline
\end{tabular}




\begin{tabular}{|c|c|c|}
\hline$U$ & auxiliary parameter & 2.1 .3 \\
\hline$\underline{u}$ & bounce point outward normal unit vector & 3.1 \\
\hline$\underline{u_{\text {simp }}}$ & bounce point outward normal unit vector, simplified model & 5.1 .1 \\
\hline$\underline{v}_{0}$ & direction of the reflected signal at a bounce point & 3.1 \\
\hline$\underline{v_{\mathrm{f}}}$ & ray-path direction from which a signal approaches a bounce point & 3.1 \\
\hline VTEC & Chapman profile's parameter of vertical total electron content & 2.3 \\
\hline$X$ & plasma-to-RF frequency ratio squared & 2.1.2 \\
\hline$\underline{X}$ & state vector & 2.1 .3 \\
\hline$\underline{x}_{\mathrm{c}}$ & $\begin{array}{l}\text { vector of the unknown receiver position components, range- } \\
\text { equivalent clock-offset and beat carrier phase measurement bias } \\
\text { terms }\end{array}$ & 3.5 .2 \\
\hline$\underline{x}_{\mathrm{f}}$ & vector of unknown parameters & 4.1.2 \\
\hline$\underline{x_{\mathrm{g}}}$ & $\begin{array}{l}\text { vector of the unknown receiver position components and range- } \\
\text { equivalent clock-offset }\end{array}$ & 3.5 .1 \\
\hline$Y$ & electron-gyro-to-RF frequency ratio & 2.1 .2 \\
\hline$y_{c}$ & measured range-equivalent beat carrier phase & 3.5 .2 \\
\hline$y_{g}$ & measured range-equivalent group delay & 3.5.1 \\
\hline$Z$ & electron/neutral-collision-to-RF frequency ratio & 2.1 .3 \\
\hline$\underline{\alpha}$ & a parameter vector & 3.2 .2 \\
\hline$\alpha$ & Gauss-Newton step scaling factor & 4.1.2 \\
\hline$\beta$ & beat carrier phase measurement bias & 3.5 .2 \\
\hline$\gamma$ & Ionosphere covariance matrix scaling factor & 7.5 \\
\hline$\underline{\Delta}$ & estimation error & 4.3 \\
\hline$\Delta p$ & a correction vector for $\underline{p}$ & 4.1 .2 \\
\hline$\underline{\Delta} \mathrm{P}$ & predicted estimation error & 4.3 \\
\hline$\Delta \underline{x}$ & a correction vector for $\underline{x}$ & 4.1 .2 \\
\hline$\Delta \eta$ & a correction vector for $\eta$ & 3.4 .1 \\
\hline$\delta$ & receiver clock's offset & 3.5.1 \\
\hline$\varepsilon$ & coefficients matrix & 2.1.2 \\
\hline$\varepsilon_{0}$ & free space dielectric constant & 2.1 .2 \\
\hline$\underline{\varepsilon} p$ & a vector of errors for the parameters of an ionosphere model & 2.3.1 \\
\hline$\zeta$ & cost function weighting coefficient & 4.1.1 \\
\hline$\underline{\eta}$ & bounce points position & 3.1 \\
\hline$\hat{\underline{\eta}}_{\mathrm{j}}$ & a set of bounce points position coordinates for the $j^{\text {th }}$ ray-path & 3.2 .1 \\
\hline$\eta_{\mathrm{m}}$ & $\begin{array}{l}\text { an ordered function vector containing coordinates for bounce points } \\
\text { position in Cartesian coordinates }\end{array}$ & 3.5.1 \\
\hline$\eta_{\text {simp }}$ & bounce points position in Cartesian coordinates, simplified model & 5.1 .2 \\
\hline$\theta$ & angle between the magnetic field and the wave vector & 2.1.2 \\
\hline$\kappa$ & measurement assessment auxiliary term & 4.2 .3 \\
\hline$\Lambda$ & a rectangular matrix of wave-lengths & 3.5.2 \\
\hline$\lambda_{w}$ & wave length & 2.1.2 \\
\hline$\lambda$ & longitude & 2.3 \\
\hline
\end{tabular}




$\begin{array}{clc}\tilde{\lambda} & \text { eigenvalue of a } 3 \times 3 \text { matrix } & 2.1 .3 \\ \mu_{0} & \text { free space permeability } & 2.1 .2 \\ v & \text { measurement residual } & 4.2 .3 \\ v_{\mathrm{c}} & \text { range-equivalent beat carrier phase measurement noise } & 3.5 .2 \\ v_{\mathrm{g}} & \text { range-equivalent group delay measurement noise } & 3.5 .1 \\ v_{m} & \text { mean electron/neutral collision frequency } & 2.1 .3 \\ v_{z} & \text { measurement noise } & 3.5 .2 \\ \xi & \text { Ionosphere Errors Index (IEI) } & 2.3 .2 \\ \underline{o} & \text { a three-term-output function } & 2.4 \\ \rho_{c} & \text { true range-equivalent beat carrier phase } & 3.5 .2 \\ \rho_{g} & \text { true range-equivalent group delay } & 3.5 .1 \\ \rho_{\mathrm{simp}} & \text { true range-equivalent group delay, simplified model } & 5.1 .2 \\ \Sigma_{1} & \text { estimation error covariance matrix } & 4.4 .1 \\ \Sigma_{2} & \text { estimation error covariance matrix } & 4.4 .2 \\ \tilde{\Sigma_{2}} & \text { estimation mean square error matrix } & 4.4 .2 \\ \tilde{\Sigma_{3}} & \text { estimation mean square error matrix } & 4.4 .4 \\ \tau & \text { normalized integration parameter } & 2.1 .3 \\ \Phi & \text { signal's phase } & 3.5 .2 \\ \phi & \text { latitude } & 2.3 \\ \Omega_{\mathrm{e}} & \text { electron gyrofrequency } & 2.1 .2 \\ \omega & \text { wave frequency } & 2.1 .2 \\ \omega 0 & \text { transmission frequency } & 2.1 .3 \\ \omega_{p} & \text { plasma frequency } & 2.1 .2 \\ & & \end{array}$




\section{CHAPTER 1}

\section{INTRODUCTION}

The use of High Frequency (HF) signals propagating in the atmosphere has been widely discussed in the literature for communications and over-the-horizon radar. Signals with frequencies in the range 2-10 MHz can bounce successively off the ionosphere and the Earth to arrive at a receiver along a Non-Line-Of-Sight (NLOS) path. Such signals have been proposed for geolocation purposes, as in Ref. [1]. The present study represents a further effort to examine the potential use of such signals for radio navigation.

Given perfect knowledge of the ionosphere and of the number of bounces between a transmitter and a receiver, it is possible to develop a model of the measured pseudorange, which is the difference between a signal's reception and transmission times multiplied by the speed of light. The pseudorange depends on the unknown user receiver location and the receiver's unknown clock offset. Given four or more such pseudoranges from four or more independent transmitters with an appropriately diverse geometry, it should be possible to solve for the unknown user position and clock offset, similar to GPS.

The problem with such an approach is that the ionosphere's HF signal propagation/refraction/reflection properties are highly uncertain due to the variability of its three-dimensional electron density distribution, $N_{e}(\underline{r})$. The approach of Ref. [1] and 
Ref. [2] is to use ionosonde data [3] in order to refine a local model of $N_{e}(\underline{r})$. This local model is then used to estimate the unknown location of a transmitter. The present approach seeks to estimate simultaneously the location of an unknown receiver, its clock offset, and corrections to relevant portions of the $N_{e}(\underline{r})$ distribution. Its fundamental input data are the measured pseudoranges, also known in literature as group delays, between an array of transmitters at known locations and the user receiver. These measurements may include multiple pseudoranges from a single transmitter for ray-paths with differing numbers of bounces or for signals with differing frequencies. A second type of measurement, known as beat carrier phase, counts carrier cycles over an arbitrary time interval and differences the resulting count with the expected nominal count for the transmitted signal waveform [4]. In the scope of this work, these measurements are used to refine the receiver's position solution once a solution that is based on pseudoranges has converged.

The approach taken in this study involves several elements. They are 1) a nominal ionosphere model, 2) estimated corrections to that model, 3) raytracing calculations for the paths of the HF signals from the transmitters to the receiver through the corrected model, and 4) model inversion calculations to estimate the user receiver position and clock offset along with the corrections to the ionosphere model. These model inversion calculations are carried out using a modified nonlinear batch least-squares solution technique.

A key question for such an approach concerns observability. Given a limited number of transmitters and a limited number of measured pseudoranges, can such a system 
accurately estimate the many unknowns? The infinite-dimensional nature of ionospheric corrections, which exist in $N_{e}(\underline{r})$ function space, theoretically dooms such an approach to failure. In practice, however, it may be possible to combine a priori information about $N_{e}(\underline{r})$ with measured pseudoranges and beat carrier phases in order to arrive at a reasonable result. This dissertation represents a study of whether this might be practically possible.

The present study follows the initial study of Ref. [5] but differs in two significant ways. The ray-path computations in Ref. [5] rely on a simplistic segmented ray-path model that is physically realistic. Second, system performance is evaluated through a series of statistical analyses that rely on a mixture of Monte Carlo calculations and computation of the theoretical linearized a posteriori solution covariance. This performance analysis assesses sensitivities to various setup parameters. In the early study, in contrast, only a limited number of test cases were examined.

As with the study presented in Ref. [5], the current study utilizes a Chapman vertical profile with horizontal variations of the three parameters that it uses to model the electron density vertical profile $N_{e}\left(h_{a l t}\right)$. This model form has known limitations that are discussed later.

The basic question of the present study concerns whether, and to what extent, the joint estimation of position, receiver clock offset, and corrections to ionosphere parameters is possible. It is well known that positioning is possible with a minimal number of received signals for the simpler satellite-based GPS problem. For the present problem, however, the increase in the number of unknowns and their complicated relationship to the 
processed measurements makes performance hard to predict based on simple analysis. Instead, performance must be studied using a truth-model simulation and a corresponding batch estimator.

This study makes three contributions to the area of radio navigation based on bouncing HF signals. First, it develops a measurement model of the pseudorange and beat carrier phase measurements of multi-hop HF signal paths from known beacon transmitter locations to an unknown user receiver location. This model includes techniques for solving its nonlinear bounce conditions and for computing first-partial derivative sensitivities of the bounces and the range measurements with respect to the unknown user location and the unknown ionosphere parameters. Second, this study develops a batch nonlinear least-squares estimation algorithm for determining the unknown user receiver position, user receiver clock offset, and ionospheric parameter corrections. This algorithm incorporates a priori information about the ionosphere parameters in order to compensate for the lack of strict simultaneous observability of the location, clock offset, and ionosphere corrections. Third, the potential performance of the proposed HF navigation scheme is evaluated using data from a truth-model simulation and using covariance analysis.

The remainder of the dissertation is divided into two parts and eight chapters. Part 1, consisting of Chapters II to V, includes theoretical background, mathematical models, a thorough description of the batch estimator, and a review of past work. Part 2, consisting of Chapters VI to VIII, presents the studies that have been performed in an effort to characterize the potential performance of the proposed system. 
Chapter II presents the physical and mathematical models of Earth, the ionosphere and the traversing HF signals.

Chapter III covers definitions and derivation of bounce points and their equations, rayhops, and ray-paths. It also discusses two measurement error models.

Chapter IV develops the batch filter that estimates the quantities of interest. It starts with formulating the governing problem and develops three different iterative solution strategies that apply in three different cases. This chapter also describes a modified Gauss-Newton method that is utilized in this study. An error analysis, including derivation of the expected estimation error, is discussed next. Chapter IV concludes with the derivation of the filter's theoretical a posteriori mean square error and covariance matrices for several different cases.

In Chapter V, past work that considered a simplified ray-path model is reviewed. This work originally indicated feasibility for the HF navigation concept, and it established the starting point for the current phase of this study. Moreover, models which have been developed during that early work are used by the current effort. This chapter concludes the first part of the dissertation.

The second part of the dissertation begins with Chapter VI, which presents various aspects of algorithm functionality and validation. It describes the truth-model simulation that has been developed to evaluate this concept, and it discusses observed batch-filtering algorithm behavior during the optimization process. Finally, it presents several analyses that concern solution convergence and covariance computations. 
Chapter VII presents methodology concepts and results for a study that considers a variety of test cases. These test cases are split into four classes, where each class consists of several groups of test cases that differ in their scenario setups. An ensemble of 21 test cases has been designed to provide an understanding of the performance that can be expected for a real system given different conditions of operation. This chapter includes tables that summarize the properties and results for most categories of test cases that have been studied and for both batch filter configurations.

Chapter VIII analyses the results that have been presented in Chapter VII. It discusses trends, observations, and findings for the collection of test cases described in the previous chapter, and it examines performance sensitivities to the various setup parameters. This analysis involves positioning accuracy, as well as the merit of the a posteriori ionosphere model. Suggestions for follow-on studies are outlined in this chapter and conclude the analytical part of this document.

Finally, Chapter IX summarizes this study's developments, and it draws conclusions about the proposed new system.

The nine chapters of this work are followed by three appendices. These consist of information that is not essential for acquiring an understanding of this project. They cover aspects of this study that certain readers may find useful. Appendix A describes the procedure of one-dimensional interpolation for a bivariate function that is used in this study. Appendix B elaborates on filter procedures and calculations that apply for the simplified ray-path model. Appendix C covers various aspects of code design and implementation. 


\section{CHAPTER 2}

\section{PHYSICAL MODELS}

\subsection{High Frequency Signals}

\subsubsection{Signal structure and ranging accuracy}

This study considers transmitted RF signals with sinusoidal carriers in the range $2 \mathrm{MHz}-8 \mathrm{MHz}$. Signals are assumed to either maintain a constant carrier frequency, or to utilize a smoothed stepping pattern for altering their carrier frequencies. In the latter case, each signal beat carrier phase is measured after a given step is complete and the signal is oscillating with a different frequency, and consequently, traversing a perturbed ray path.

The basic ranging procedure relies on Binary Phase-Shift Keying (BPSK) modulated codes or some similar spread-spectrum technique. The resulting accuracy for this sort of ranging in terms of measurement noise 1-sigma is about one kilometer assuming a signal bandwidth of $100 \mathrm{KHz}$. Carrier phase measurements are assumed to be derived using an accurate internal oscillator and phase-lock loop so that the expected accuracy for a beat carrier phase measurement is one meter based on extrapolation of the fraction of a cycle of phase that can be resolved for L-band signals using standard GPS signal-processing techniques. It remains an open question whether such results can be achieved for HF signals. 


\subsubsection{Physical characteristics of wave propagation in the ionosphere}

A crucial component in the process of solving the governing positioning/timing estimation problem is the ability to accurately determine and reconstruct trajectories for the HF signals that traverse a modeled ionosphere. These trajectories are characterized not only by their curved shape, but also by the frequency- and path-dependent propagation speeds of their BPSK modulated code and carrier wave. Propagation speed dependence on wave frequency is known as dispersive wave propagation and is typical of propagation in the ionosphere [3], [6] and [7].

The long-term propagation mechanism that is utilized in this study relies on ionospheric refraction that bends skyward-propagating radio waves back towards the Earth in a way that somewhat resembles reflection. This effect can occur for signals in the frequency range of up to $40 \mathrm{MHz}$ [8]. Long distance propagation during daytime cannot be sustained for wave frequencies less than $2 \mathrm{MHz}$ due to strong absorption in the D layer [9].

When neutral atoms and molecules in the upper atmosphere are subjected to strong ultraviolet radiation, they may be ionoized into plasma - a state of matter consisting of a varying concentration of electrons and ions due to ultra-violet radiation from the Sun. Plasmas are electrically conductive and hence respond strongly to electromagnetic fields. Conductivity modifies the index of refraction of the medium, causing RF waves to reflect and refract. An important property of the resulting ionized substance is known as the plasma frequency and is denoted $\omega_{p}$. The plasma frequency corresponds to a natural 
oscillation frequency of free electrons. For an electron density of $N_{e}(\underline{r})$, the plasma frequency is given by

$$
\omega_{p}=\sqrt{N_{e}(\underline{r}) q_{e}^{2} / m_{e} \varepsilon_{0}}
$$

where $q_{e}, m_{e}$ and $\varepsilon_{0}$ are, respectively, the electron charge, the electron mass and the free space dielectric constant.

Let $\omega$ denote the wave frequency and let $k \equiv 2 \pi / \lambda_{w}(=\omega / c)$ denote the wave number. The phase velocity is in the direction of the wave vector $\underline{k}$ which is normal to the direction of the electric displacement. Group velocity is in the direction of the Poynting flux, which is normal to the direction of the electric field [10].

A precise analysis of the behavior of electromagnetic waves as they traverse through plasma is conducted through the combining of Faraday's law and Ampere's law in macroscopic form, where the wave solution takes the form

$$
A_{0} \exp (j(\omega t-\underline{k} \cdot \vec{r}))
$$

with the wave vector $\underline{k}$ being perpendicular to both magnetic field and electric displacement field but not necessarily to the electric field, $\underline{E}$. Combining Maxwell's equations yields the wave equation

$$
\underline{k}(\underline{k} \cdot \underline{E})-k^{2} \underline{E}+\omega^{2} \mu_{0} \varepsilon \cdot \underline{E}=\underline{0}
$$


where $\mu_{0}$ is the free space permeability and $\varepsilon$ is a $3 \times 3$ permittivity matrix that consists of terms dependent on $\omega, \omega_{p}$ and the electron gyrofrequency $\Omega_{\mathrm{e}}$, which is dependent on Earth's magnetic field $\underline{B}$ through

$$
\Omega_{e}=\frac{e\|\underline{B}\|}{m_{e}}
$$

Eq. (3) can be written as the linear homogeneous system

$$
K(\underline{k}) \underline{E}=\underline{0}
$$

in the unknown $\underline{k}$ and where $K(\underline{k})$ is a $3 \times 3$ matrix. Desired solutions in a form relating $\underline{k}$ and the direction of $\underline{E}$ are obtained by setting $K$ 's determinant equal zero. These solutions correspond to the different polarization modes. Typically, one should expect two independent solutions for any given wave frequency and propagation direction. The two modes will have distinct, close to orthogonal polarization and will be governed by different dispersion relations.

It is common practice to formulate the wave normal equation in terms of $\theta$, the angle between the magnetic field $\underline{B}$ and the wave vector $\underline{k}$, and in terms of the index of refraction, $n$. One such formulation is known as Astrom's Equation. It is given by

$$
\begin{aligned}
& \left(\frac{n_{1}^{2}}{n^{2}-n_{1}^{2}}+\frac{n_{-1}^{2}}{n^{2}-n_{-1}^{2}}\right) \frac{\sin ^{2}(\theta)}{2}+\frac{n_{0}^{2}}{n^{2}-n_{0}^{2}} \cos ^{2}(\theta)=0 \\
& n_{\alpha}{ }^{2} \equiv 1-\frac{X}{1-\alpha^{\prime} Y} ; \alpha^{\prime}=-1,0,1 ; X \equiv \omega_{p}^{2} / \omega^{2} ; Y \equiv \Omega_{e} / \omega
\end{aligned}
$$

The Booker Quartic formulation is equivalent to Astrom's Equation. It takes the form 


$$
\left[n_{1}^{2}\left(n^{2}-n_{-1}^{2}\right)+n_{-1}^{2}\left(n^{2}-n_{1}^{2}\right)\right] \frac{\sin ^{2}(\theta)}{2}+n_{0}^{2}\left(n^{2}-n_{1}^{2}\right)\left(n^{2}-n_{-1}^{2}\right) \cos ^{2}(\theta)=0
$$

Eq. (7) can be factored as a closed form solution for $\mathrm{n}^{2}$. This formula is known as the Appleton-Hartree formula

$$
\begin{aligned}
& n^{2}=1-\frac{X}{1-\frac{Y_{T}^{2}}{2(1-X)} \pm \sqrt{\frac{Y_{T}^{4}}{4(1-X)^{2}}+Y_{L}^{2}}} \\
& Y_{T}=Y \sin (\theta) ; Y_{L}=Y \cos (\theta)
\end{aligned}
$$

Waves propagate when $n^{2}>0$. Eqs. (6), (7) and (8) can be used to determine wave frequencies at which wave propagation ceases. These frequencies, known as cutoff frequencies, are determined by setting $n^{2}=0$. The two solutions, $\omega_{1}$ and $\omega_{2}$, obey the equations $\omega_{1}{ }^{2}=\omega_{\mathrm{p}}{ }^{2}$ and $\omega_{\mathrm{p}}{ }^{2}=\omega_{2}\left(\omega_{2}-\Omega_{e}\right)$. These are the cutoff frequencies for the ordinary $(\mathrm{O})$ and extraordinary $(\mathrm{X})$ wave modes, respectively. The characteristic polarizations for the two modes are generally elliptical. However, when propagating in a direction that is close to perpendicular to the magnetic field, the $O$ mode is characterized by linear polarization with its electric field parallel to the axis of the background magnetic field. The electric field of the extraordinary wave mode is approximately perpendicular to background magnetic field when propagating in a direction that is perpendicular to the magnetic field. The manner in which polarization changes throughout signal propagation is beyond the scope of this discussion. See Ref. [11] for a discussion of polarization. 


\subsubsection{Raytracing}

Raytracing calculations lie at the core of this study. The ability to accurately model signal trajectories is essential to the success of the estimation process. Calculations are based on a numerical solution to the wave equations. This subsection discusses the basics of these computations.

Ray tracing is carried out through numerical propagation of Hamilton's equations that apply for a propagating RF signal in an ionized medium. The fundamental set of equations is provided by Ref. [12] in the form of non-linear ordinary differential equations (Eqs. 9 through 15 of that paper) that can be written as:

$$
\begin{aligned}
& \frac{d \underline{r_{w}}}{d P^{\prime}}=-\left[\frac{d H}{d \underline{k}}\right]^{T} / c \frac{d H}{d \omega}=f_{1}\left(\underline{r_{w}}, \underline{k}\right) \\
& \frac{d \underline{k}}{d P^{\prime}}=\left[\frac{d H}{d \underline{r_{w}}}\right]^{T} / c \frac{d H}{d \omega}=f_{2}\left(\underline{r_{w}}, \underline{k}\right)
\end{aligned}
$$

$H$ is the Hamiltonian and the independent variable $P^{\prime} \equiv c t_{g}$ is the range-equivalent group delay parameter that takes the value $P^{\prime}{ }_{0}$ at the beginning of the trajectory and $P_{\mathrm{f}}^{\prime}$ at its end. The same Hamiltonian can be used to develop a differential equation for the range-equivalent carrier phase $P=\phi / k_{0}$ with $\phi$ being the carrier phase in radians. This differential equation takes the form

$$
\frac{d P}{d P^{\prime}}=-\frac{d H}{d \underline{k}} \underline{k} / \frac{d H}{d k_{0}} k_{0}
$$

where $k_{0}=\omega / c$ is the free-space wave number with $\omega$ being the transmission frequency. 
Reference [12], which combines the work of Ref. [13] and Ref. [14], gives several Hamiltonians that can be used in Eq. (9). They are generally based on the AppletonHartree formula of Eq. (8) or on a closely related derivation. The following formulation for the Hamiltonian is used with most sub-routines that are utilized in that paper.

$$
H=\operatorname{real}\left\{\frac{1}{2}\left(\frac{c^{2}}{\omega^{2}}\left(k_{x}^{2}+k_{y}{ }^{2}+k_{z}^{2}\right)-n^{2}\right)\right\}
$$

where $k_{x}, k_{y}$, and $k_{z}$ are the three Cartesian components of the wave vector $\underline{k}$. This wave vector notation will be used hereafter for Hamiltonian formulation. A second Hamiltonian that is based on Ref. [15]. It takes the form

$$
\begin{aligned}
H & =\operatorname{real}\left\{\left[(U-X) U^{2}-Y^{2} U\right] c^{4} k^{4}+X(k \cdot Y)^{2} c^{4} k^{2}\right\} \\
& +\operatorname{real}\left\{\left[-2 U(U-X)^{2}+Y^{2}(2 U-X)\right] c^{2} k^{2} \omega^{2}-X(k \cdot Y)^{2} c^{2} \omega^{2}\right\} \\
& +\operatorname{real}\left\{\left[(U-X)^{2}-Y^{2}\right](U-X) \omega^{4}\right\}
\end{aligned}
$$

where $U \equiv 1-j Z, Z \equiv v_{m} / \omega$ and $v_{m}$ is the mean electron/neutral collision frequency. The Hamiltonian of Eq. (11) is not appropriate high up in the ionosphere near any possible spitze of the ray path because it can become singular in this region. This is the region were Ref. [12] suggests the use of the Hamiltonian of (12) instead. The latter Hamiltonian, however, would function poorly when in or near free space, where all of its derivatives become very close to zeroes.

Alternative Hamiltonian formulations are presented in Ref. [16]. These are the Hamiltonians that are utilized with the current implementation of this study. The first Hamiltonian, which is used where the electron density is relatively small, is given by 


$$
\begin{aligned}
& H=\frac{1}{2} \operatorname{real}\left\{\left[\left(\frac{\underline{k}^{T} \underline{k}}{k_{0}^{2}}\right)-n_{A H}{ }^{2}\left(\underline{r_{w}}, \frac{\underline{k}}{\|\underline{k}\|}, k_{0}, \underline{p}\right)\right]\right. \\
& \times {\left.\left[(1-j Z)^{2}-Y^{2}\right](1-j Z)\right\} }
\end{aligned}
$$

where $\underline{p}$ is a vector of parameters that characterizes the ionosphere electron density profile. $n_{\mathrm{AH}}$ is the lossy Appleton-Hartree index of refraction of Ref. [17].

A different Hamiltonian is used near a reflection point/spitze. It does not experience any singularities in this vicinity. This Hamiltonian takes the form

$$
H=\frac{1}{2} \operatorname{real}\{\tilde{\lambda}\}
$$

where $\tilde{\lambda}$ is an eigenvalue of the $3 \times 3$ matrix $\hat{D}$ that is given by

$$
\begin{aligned}
\hat{D}= & {\left[\left(\frac{k^{T}}{k_{0}^{2}}-1\right) I-\frac{k k^{T}}{k_{0}{ }^{2}}\right] \times\left[(1-j Z)^{2}-Y^{2}\right](1-j Z) } \\
& +X\left\{(1-j Z)^{2} I-Y^{2} \hat{B}_{0} \hat{B}_{0}^{T}-j(1-j Z) Y\left[\hat{B}_{0} \times\right]\right\}
\end{aligned}
$$

$\hat{B}_{0}$ is a unit vector that points in the direction of the ambient geomagnetic filed vector, $\underline{B}$.

A state space system of equations is defined for the unknown wave-front position and wave-vector :

$$
\begin{aligned}
& \underline{\dot{\tilde{X}}}=\underline{\tilde{f}}(\underline{\tilde{X}}) \\
& \tilde{\tilde{f}}=\left[\begin{array}{l}
f_{1}(\underline{\tilde{X}}) \\
f_{2}(\underline{\tilde{X}})
\end{array}\right] ; \quad \underline{\tilde{X}}=\left[\begin{array}{l}
\underline{r}_{w} \\
\underline{k}
\end{array}\right]
\end{aligned}
$$


This state vector consists of the three Cartesian coordinates of the propagating wave front's position $\underline{r}_{w}$ and the three components of the wave vector $\underline{k}$. For practical reasons, the state vector that is used with the current numerical implementation is defined in the normalized form:

$$
\underline{X}=\left[\begin{array}{c}
\underline{r}_{w} / P_{f}^{\prime} \\
\underline{k} / k_{0}
\end{array}\right]
$$

Normalization of the first term by $P_{f}^{\prime}$ and of the second term by $k_{0} \equiv \omega / c$ results in a unitless state vector, whose derivative with respect to the parameter $\tau \equiv P^{\prime} / P_{f}^{\prime}$ is given by:

$$
\frac{d \underline{X}}{d \tau}=P_{f}^{\prime} \frac{d \underline{X}}{d P^{\prime}}=\underline{f}\left(\underline{X}, \underline{p}, P_{f}^{\prime}\right)
$$

$f$ can be numerically propagated from the initial $\tau=0$ to the final $\tau=1$. The terminal value $P_{f}^{\prime}$ is unknown and must be determined as part of a two-point boundary value problem solution. Integration is performed using a $4^{\text {th }} / 5^{\text {th }}$ order Runge-Kutta method, and its Runge-Kuta grid points are revised until a sufficient level of accuracy is achieved through a process of step size adaptation. Further details on the raytracing process implementation are given in Section III of Ref. [16].

\subsection{The Earth Model}

Models of the Earth and the ionosphere are used to define the physical environment for the propagating signals. These models have been chosen so that they combine the need for a reasonably realistic representation of physical phenomena with the need to 
limit the complexity of the models and the resulting computational effort for purposes of the present study. Models with increased fidelity and increased complexity would be required for the processing of actual HF signal data to produce accurate navigation solutions.

\subsubsection{Earth geometry model}

An Earth surface geometry model is needed for two reasons. The first use is to define the bounce conditions of HF ray paths at the Earth's surface. The second use is to define altitude within the calculations of this study's ionosphere model.

The Earth is modeled as a closed, continuous and smooth surface that is known as the WGS-84 ellipsoid [18]. The implicit equation for the ellipsoid in Cartesian Earth Centered Earth Fixed (ECEF) coordinates is

$$
\frac{r_{1}^{2}}{R_{c}^{2}}+\frac{r_{2}^{2}}{R_{c}^{2}}+\frac{r_{3}^{2}}{R_{P}^{2}}=1 ; R_{c}=6378137 \quad ; \quad R_{P}=6356752.31425
$$

Where $r_{1}, r_{2}$ and $r_{3}$ are coordinates in meters.

The function $h_{a l t}(\underline{r})=h_{W G S 84}\left(r_{1}, r_{2}, r_{3}\right)$ computes the altitude of a Cartesian ECEF point $\left(r_{1}, r_{2}, r_{3}\right)$ above the WGS-84 ellipsoid, as in Ref. [19]. All points of the form $\left(r_{I_{k}}, r_{2}{ }_{k}, r_{3}\right)$ on the ellipsoid therefore satisfy $h_{\text {alt }}\left(\underline{\underline{r}}_{k}\right)=0$. The gradient of $h_{\text {alt }}\left(\underline{x}_{k}\right)$ is of interest, as will be presented later in the context of bounce points.

This approach for modeling the Earth has been chosen for its relative simplicity and the fact that it does not rely on availability of additional data. A more realistic method for 
modeling the shape of the Earth would use an existing digital representations of the Earth. A commonly used form of representation is a Digital Terrain Model (DTM) or Digitial Elevation Map (DEM). Such a model could be used to develop a bi-quintic spline for describing the latitude/longitude dependent altitude. The data required for DTM/DEM-based modeling is available for national agencies and institutes - see Ref. [20].

A DTM/DEM model would provide a higher fidelity calculation of the bounce conditions for reflection of HF waves off of the ground. For purposes of the truth-model simulation study, however, a DTM/DEM model does not provide a significant advantage over a simple WGS-84 ellipsoid because the nature of the signal bounces off of the Earth does not change markedly between the two models except in very mountainous regions. The WGS-84 model has the advantage of simpler calculations. That is why it is used in the present simulation-based study. A DTM/DEM-based model should be used for any proposed application of this dissertation's methods to real data.

\subsubsection{Earth magnetic field model}

Raytracing computations for propagating HF signals require knowledge of the Earth's magnetic flux vector field at any desired location. This study uses the $11^{\text {th }}$ generation model for the International Geomagnetic Reference Field (IGRF), known as IGRF-11, for which the magnetic flux is modeled as the gradient of a time-varying spatial potential function. Additional information on this model can be found in Ref. [21]. 


\subsection{The Ionosphere Model}

This region of Earth's upper atmosphere is characterized by ionization that is caused by ultraviolet solar radiation. The majority of the ions in the ionosphere are photoionization dislodged dinitrogen and dioxygen molecules. A roughly equal number of free electrons is assumed to lie in close proximity to the ionized molecules so that the total charge of the medium is assumed nearly neutral.

A three-parameter Chapman beta model is used to model the location-dependent electron density distribution of the ionosphere. This model regards the ionosphere as a medium with an altitude-dependent electron density whose altitude density distribution is characterized by parameters that vary with latitude and longitude. For a given time of interest $t$, electron density is given by

$$
\begin{aligned}
& N_{e}(\underline{r}, t)=\frac{V T E C[\phi(\underline{r}), \lambda(\underline{r}), t]}{e \cdot h_{s f}[\phi(\underline{r}), \lambda(\underline{r}), t]} \exp (1-z(\underline{r}, t)-\exp (-z(\underline{r}, t))) \\
& z(\underline{r}, t)=\frac{h_{\text {alt }}(\underline{r})-h_{\max }[\phi(\underline{r}), \lambda(\underline{r}), t]}{h_{s f}[\phi(\underline{r}), \lambda(\underline{r}), t]}
\end{aligned}
$$

where $\phi(\underline{r}), \lambda(\underline{r})$ and $h_{\text {alt }}(\underline{r})$ are, respectively, the latitude, longitude, and altitude above the WGS-84 ellipsoid of the ECEF position $\underline{r} . \quad N_{e}(\underline{r}, t)$ is given in units of electrons $/ \mathrm{m}^{3}$. The quantity $h_{\max }[\phi(\underline{r}), \lambda(\underline{r}), t]$ is the altitude of the maximum electron density of the Chapman profile. The quantity $\operatorname{VTEC}[\phi(\underline{r}), \lambda(\underline{r}), t]$ is the vertical total electron content - the integral of the electron density along a vertical path. The quantity $h_{s f}[\phi(\underline{r}), \lambda(\underline{r}), t]$ is the Chapman profile's altitude scale height. 
It should be noted that the three Chapman parameters are time dependent. They usually vary slowly in time due to the daily variations of the amount of solar radiation and other effects. While the rate of change is small enough for these parameters to be regarded as constants during the short duration of signal propagation in the context of this project, the differences in their values for time intervals that are in the order of an hour or more are significant. In particular, diurnal, seasonal, and solar effects make the resulting time variations very significant.

The simplistic Chapman model ignores the possibility of distinct $\mathrm{D}$ and $\mathrm{E}$ layers, including a sporadic E layer. This level of simplification would likely produce unsatisfactory results if working with daytime real data, but it is reasonable to use a Chapman profile at this stage of simulation-based study of the proposed system's potential accuracy.

The natural logarithm of the latitude/longitude variations of the three Chapman vertical profile parameters are modeled using bi-quintic splines as described in Subsection 2.3.1. The spline nodes are placed at predefined latitudes and longitudes with subsets of nodes grouped into common small circles of constant latitude. Figure 1 illustrates the placement of the grid nodes, where each grid node is identified by a unique number, starting at one for the node that is located at the south pole and ending at 424 for the node that is located at the north pole. The set of grid nodes that is used with this study has been defined in a way that gives a sufficient number of nodes over North America, the simulated region of interest. The number of nodes used outside this region is 
somewhat arbitrary and is probably larger than it needs to be. In general, other node point selections are possible.

The set of parameters for each grid node are the given function's value and eight partial derivatives with respect to latitude $\phi$ and longitude $\lambda$. Thus, a vector of nine parameters $\underline{p}_{a, i}$ is associated with the $i^{\text {th }}$ node and the splined scalar function $a(\phi, \lambda)$ as follows:

$$
\underline{p}_{a, i}=\left[a, \frac{\partial a}{\partial \lambda}, \frac{\partial a}{\partial \phi}, \frac{\partial^{2} a}{\partial \lambda^{2}}, \frac{\partial^{2} a}{\partial \lambda \partial \phi}, \frac{\partial^{2} a}{\partial \phi^{2}}, \frac{\partial^{3} a}{\partial \lambda^{2} \partial \phi}, \frac{\partial^{3} a}{\partial \lambda \partial \phi^{2}}, \frac{\partial^{4} a}{\partial \lambda^{2} \partial \phi^{2}}\right]^{T}
$$

Given the latitude $\phi_{0}$ and the longitude $\lambda_{0}$ of a point at which one wants to compute the value of the natural logarithm of a Chapman parameter, $a$, (and possibly various of its partial derivatives), the needed calculations use the nearest four bi-quintic spline nodes that lie northwest, northeast, southwest, and southeast of $\left(\phi_{0}, \lambda_{0}\right)$. Stated differently, these four points lie on the two small circles of latitude which bracket $\phi_{0}$. On each of these two small circles, the two chosen node points are those whose longitudes bracket $\lambda_{0}$.

Three sets of vectors $\underline{p}_{a, 1}$ to $\underline{p}_{a, 424}$, each set computed for a different Chapman parameter, can be used to fit the International Reference Ionosphere (IRI) model. First a Chapman vertical profile is fit to the IRI vertical profile at each spline node point to give

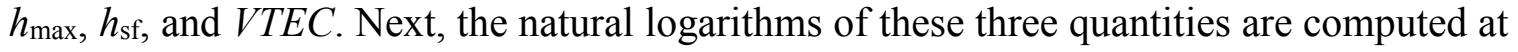
each node point. Finally, a smoothness criterion is employed to determine the various partial derivatives of these quantities for inclusion in $\underline{p}_{a, \mathrm{i}}$ of Eq. (21) using a spline 
initialization method found in Ref. [22]. Further details on this procedure are given in Subsection 6.1.2.

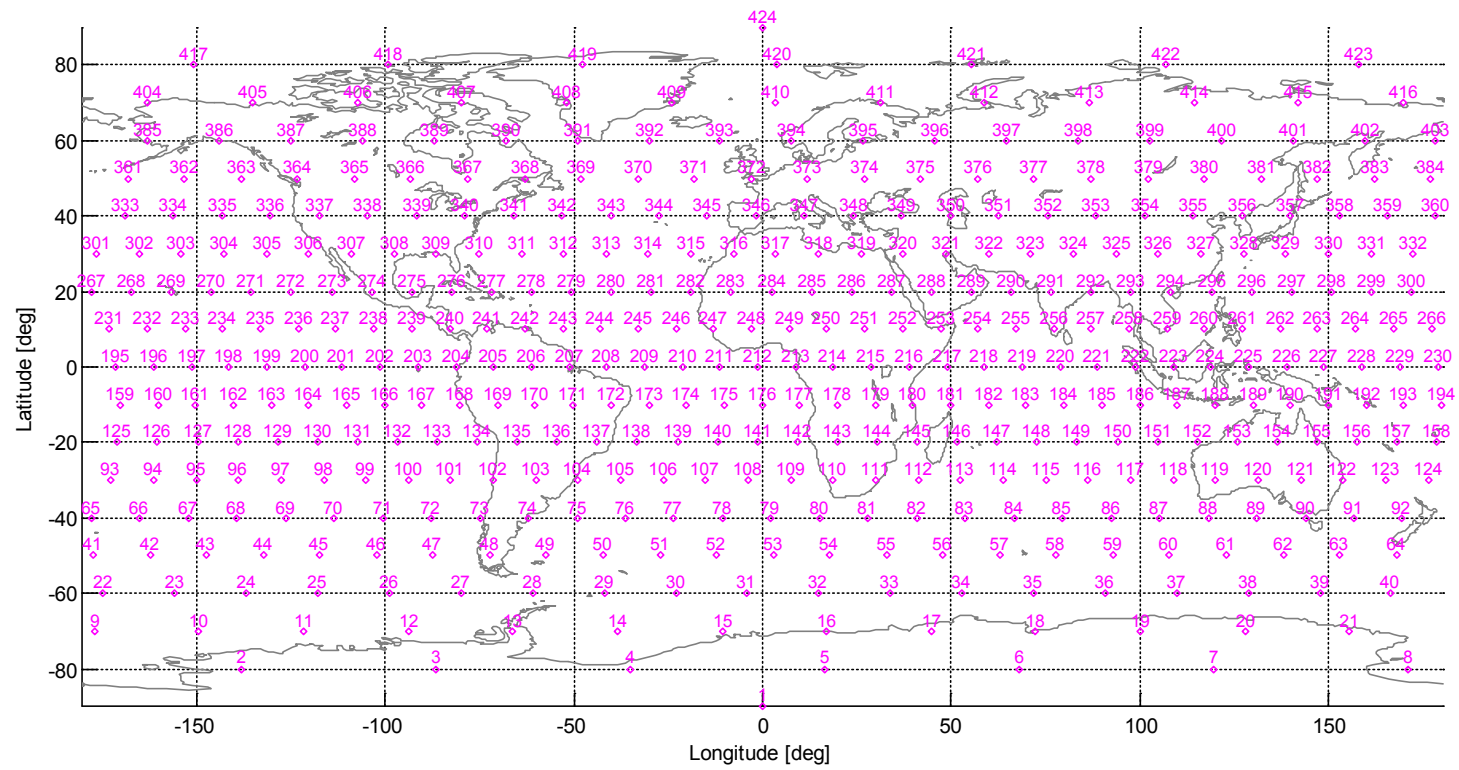

Figure 1: The 424 grid nodes placement map for an example latitude/longitude bi-quintic spline.

\subsubsection{Ionosphere parameter errors and the $M$ covariance matrix}

An error model is considered for the a priori ionosphere parameters. These errors reflect a Chapman based representation of imperfect knowledge of the true spatial electron distribution in the ionosphere. For the general case, the error vector $\underline{\varepsilon}_{p}$ is defined through

$$
\underline{\bar{p}}=\underline{p}_{\text {true }}+\underline{\varepsilon}_{p}
$$

where $p_{\text {true }}$ is the vector of parameters that define the true ionosphere and $\bar{p}$ contains $a$ priori estimates of the ionosphere parameters. Both vectors are stacked versions of the elementary vectors $\underline{p}_{a, \mathrm{i}}$ so that 


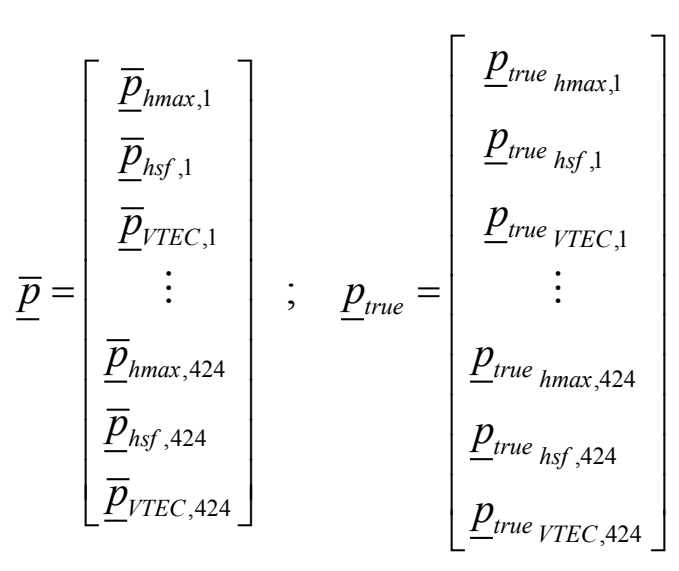

The nature of the a priori model error vector $\underline{\varepsilon}_{p}$ is determined by the case. It is modeled as either a vector of constants or as a sample from a multivariable Gaussian distribution, as discussed later in the context of navigation accuracy analysis.

In order to model the likely correlations between the various terms of $\overline{\underline{p}}$, the IRI model was used to compute the best-fit Chapman parameter values four times a day throughout the calendar year 2009 , where the first sampling time in hours is a uniformly distributed random variable in the range $[0,6]$ and the following three times are spaced six hours apart. See Ref. [23] and Ref. [24] on current IRI modeling, and Ref. [25] on further improvement efforts. Reference [26] describes the Chapman parameter fitting procedure. This method of sampling was chosen in order to minimize possible effects of an hourlyscale periodicity. The resulting 1460 parameter vectors were used to compute an empirical covariance matrix for the natural logarithm of all Chapman model based ionospheric parameters of Eq. (21). This matrix is defined as the ionospheric parameters' a priori covariance matrix, designated $M_{0}$ throughout this dissertation. 


\subsubsection{The Ionosphere Errors Index}

It will be useful later in this dissertation to have a quantitative measure for the deviation of a given set of ionosphere parameters, such as the a-priori ionosphere model, from their "true" values within a truth-model simulation. The unitless Ionosphere Errors Index (IEI) is a scalar quantity that is defined as follows.

$$
\xi=\log _{10}\left(\frac{1}{2 N_{p}} \underline{\varepsilon}_{p}^{T} M^{-1} \underline{\varepsilon}_{p}\right)
$$

where $N_{p}$ is the number of ionosphere parameters that are associated with applicable grid nodes, i.e., grid nodes that define grid cells through which propagating rays travel, and where $M$ is a covariance matrix that was constructed from the $M_{0}$ matrix by considering rows and columns that are associated with this same set of parameters.

An understanding of how $\xi$ is statistically distributed is desirable when assessing receiver/transmitters/ionospheric model setups. As an example, a test case is considered that has characteristics similar to those of Test Case A0 that later takes a role in the results presentation of Chapter 7, with the same receiver/transmitters setup and the same true time of 10-23-2009 at 14:22 UTC. This setup results in 13 active grid nodes and consequently, in a set of $13 \times 3 \times 9=351$ applicable ionosphere parameters. $\xi$ was computed for this subset of ionosphere parameters for all 1460 time instances spread over a year that are mentioned in the previous subsection. The above time was used as the reference time in this computation. It should be noted that none of the 1460 time instances is identical to the reference time. The resulting 1460 values of $\xi$ are distributed in a way that is illustrated in Fig. 2. 
The top panel of Fig. 2 plots computed $\xi$ values versus the time difference in days between the 1460 sample times and the reference time. The red curve is a smoothed variant of the blue curve. Unsurprisingly, smaller values were computed near zero time difference. However, an important observation is that larger time discrepancies do not necessarily result in larger IEI values. This was the motivation for abandoning the absolute seasonal discrepancy criterion of Ref. [5].
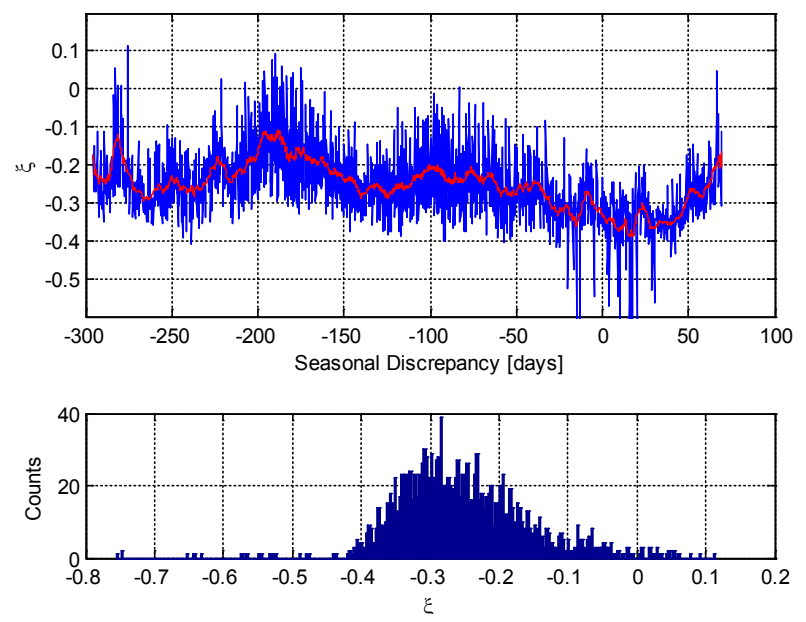

Figure 2: IEI values vs. time difference (top); IEI histogram (bottom).

The bottom panel of Fig. 2 plots a histogram of all 1460 computed values of $\xi$. Since the ionosphere parameters' error vector is normalized by its covariance matrix inverse and the number of parameters, it can be expected that $\xi$ will have a mean value close to $\log _{10}(1 / 2)=-0.3$ (see Eq. (24)). In practice, the mean value is about -0.25 . In the later discussion that follows, $\xi$ values that lie to the left of this mean will be regarded as 'small' $\xi$. For such values, the truth and the a priori ionosphere are somewhat consistent. At the same time, $\xi$ values that lie to the right of this mean will be regarded as 'large' $\xi$. 
For these cases, the a priori ionosphere model's differences from the 'truth' ionosphere are considered to be large in a statistical sense.

\subsection{Bi-quintic spline calculations}

This project utilizes bi-quintic spline modeling and calculations for two purposes:

a) Representation of the natural logarithm of the latitude/longitude dependent $h_{\max }$, $h_{s f}$ and VTEC Chapman parameters, as discussed previously.

b) Representation of the $80 \%$ electron density height surface, as discussed in Subsection 3.4.2.

Figure 3 illustrates the setup for the bi-quintic spline calculation for an unknown quantity of interest, $a$, at point $C_{0}$ of latitude $\phi_{0}$ and longitude $\lambda_{0}$.

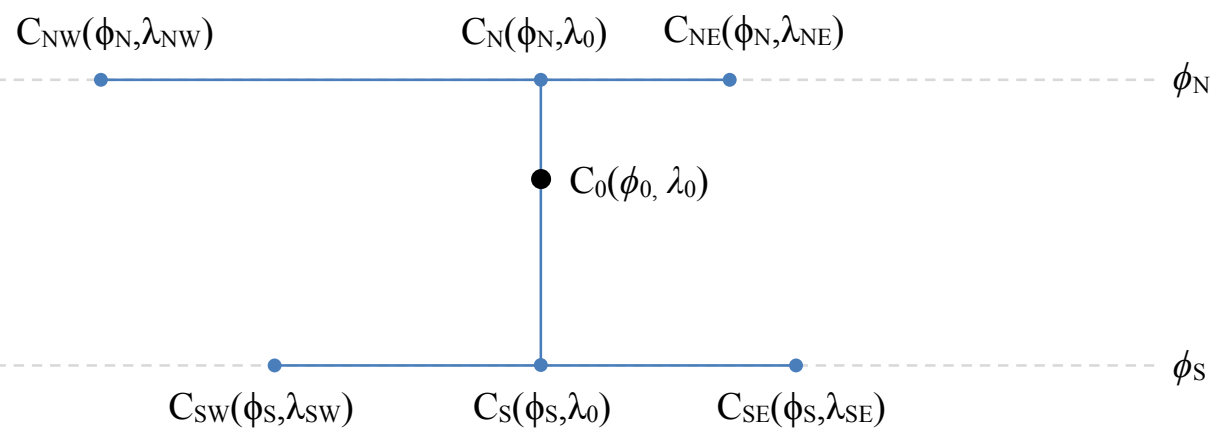

Figure 3: The bi-quintic spline calculation latitude/longitude grid example.

The $a\left(\phi_{0}, \lambda_{0}\right)$ bi-quintic spline calculations proceed as follows: Suppose that $C_{\mathrm{SW}}\left(\phi_{S}, \lambda_{\mathrm{SW}}\right)$ and $C_{\mathrm{SE}}\left(\phi_{S}, \lambda_{\mathrm{SE}}\right)$ are the neighboring southwest and southeast biquintic spline nodes and that $C_{\mathrm{NW}}\left(\phi_{\mathrm{N}}, \lambda_{\mathrm{NW}}\right)$ and $C_{\mathrm{NE}}\left(\phi_{\mathrm{N}}, \lambda_{\mathrm{NE}}\right)$ are the neighboring northwest and northeast 
nodes. Then $\phi_{S} \leq \phi_{0} \leq \phi_{N}, \lambda_{\mathrm{SW}} \leq \lambda_{0} \leq \lambda_{\mathrm{SE}}$, and $\lambda_{\mathrm{NW}} \leq \lambda_{0} \leq \lambda_{\mathrm{NEE}}$. The values for $a$ and two of its derivatives at point $C_{\mathrm{N}}\left(\phi_{N}, \lambda_{0}\right)$ are obtained with the three-term-output function $\underline{o}$ through

$$
\begin{aligned}
& {\left[a\left(C_{N}\right) \frac{\partial a}{\partial \lambda}\left(C_{N}\right) \frac{\partial^{2} a}{\partial \lambda^{2}}\left(C_{N}\right)\right]^{T}=} \\
& \underline{o}\left(\lambda_{N W}, a\left(C_{N W}\right), \frac{\partial a}{\partial \lambda}\left(C_{N W}\right), \frac{\partial^{2} a}{\partial \lambda^{2}}\left(C_{N W}\right), \lambda_{N E}, a\left(C_{N E}\right)\right. \\
& \left., \frac{\partial a}{\partial \lambda}\left(C_{N E}\right), \frac{\partial^{2} a}{\partial \lambda^{2}}\left(C_{N E}\right), \lambda_{0}\right)
\end{aligned}
$$

where $\underline{o}$ is a three-term-output function whose description is given in Appendix A. Similarly, the values for $\partial a / \partial \phi$ and $\partial^{2} a / \partial \phi^{2}$ and their first and second partial derivatives with respect to $\lambda$ are obtained through

$$
\begin{aligned}
& {\left[\frac{\partial a}{\partial \phi}\left(C_{N}\right) \quad \frac{\partial^{2} a}{\partial \phi \partial \lambda}\left(C_{N}\right) \frac{\partial^{3} a}{\partial \phi \partial \lambda^{2}}\left(C_{N}\right)\right]^{T}=} \\
& \underline{o}\left(\lambda_{N W}, \frac{\partial a}{\partial \phi}\left(C_{N W}\right), \frac{\partial^{2} a}{\partial \phi \partial \lambda}\left(C_{N W}\right), \frac{\partial^{3} a}{\partial \phi \partial \lambda^{2}}\left(C_{N W}\right), \lambda_{N E}, \frac{\partial a}{\partial \phi}\left(C_{N E}\right)\right. \\
& \left., \frac{\partial^{2} a}{\partial \phi \partial \lambda}\left(C_{N E}\right), \frac{\partial^{3} a}{\partial \phi \partial \lambda^{2}}\left(C_{N E}\right), \lambda_{0}\right)
\end{aligned}
$$

and

$$
\begin{aligned}
& {\left[\frac{\partial^{2} a}{\partial \phi^{2}}\left(C_{N}\right)\right.}\left.\frac{\partial^{3} a}{\partial \phi^{2} \partial \lambda}\left(C_{N}\right) \frac{\partial^{4} a}{\partial \phi^{2} \partial \lambda^{2}}\left(C_{N}\right)\right]^{T}= \\
& \underline{o}\left(\lambda_{N W}, \frac{\partial^{2} a}{\partial \phi^{2}}\left(C_{N W}\right), \frac{\partial^{3} a}{\partial \phi^{2} \partial \lambda}\left(C_{N W}\right), \frac{\partial^{4} a}{\partial \phi^{2} \partial \lambda^{2}}\left(C_{N W}\right), \lambda_{N E}, \frac{\partial^{2} a}{\partial \phi^{2}}\left(C_{N E}\right)\right. \\
&\left., \frac{\partial^{3} a}{\partial \phi^{2} \partial \lambda}\left(C_{N E}\right), \frac{\partial^{4} a}{\partial \phi^{2} \partial \lambda^{2}}\left(C_{N E}\right), \lambda_{0}\right)
\end{aligned}
$$


Similar calculations are carried out to obtain $a\left(C_{S}\right), \partial a / \partial \lambda\left(C_{S}\right), \partial^{2} a / \partial \lambda^{2}\left(C_{S}\right), \partial a / \partial \phi\left(C_{S}\right)$, $\partial^{2} a / \partial \phi \partial \lambda\left(C_{S}\right), \partial^{3} a / \partial \phi \partial \lambda^{2}\left(C_{S}\right), \partial^{2} a / \partial \phi^{2}\left(C_{S}\right), \partial^{3} a / \partial \phi^{2} \partial \lambda\left(C_{S}\right)$ and $\partial^{4} a / \partial \phi^{2} \partial \lambda^{2}\left(C_{S}\right)$ using the spline node parameters at points $C_{\mathrm{SW}}$ and $C_{\mathrm{SE}}$. This set of computed values and partial derivatives for points $C_{\mathrm{N}}$ and $C_{\mathrm{S}}$ can now be used to compute the desired values at $C_{0}$ as follows:

$$
\begin{aligned}
& {\left[a\left(C_{0}\right) \frac{\partial a}{\partial \phi}\left(C_{0}\right) \frac{\partial^{2} a}{\partial \phi^{2}}\left(C_{0}\right)\right]^{T}=} \\
& \underline{o}\left(\phi_{S}, a\left(C_{S}\right), \frac{\partial a}{\partial \phi}\left(C_{S}\right), \frac{\partial^{2} a}{\partial \phi^{2}}\left(C_{S}\right), \phi_{N}, a\left(C_{N}\right), \frac{\partial a}{\partial \phi}\left(C_{N}\right), \frac{\partial^{2} a}{\partial \phi^{2}}\left(C_{N}\right), \phi_{0}\right) \\
& {\left[\frac{\partial a}{\partial \lambda}\left(C_{0}\right) \frac{\partial^{2} a}{\partial \lambda \partial \phi}\left(C_{0}\right) \frac{\partial^{3} a}{\partial \lambda \partial \phi^{2}}\left(C_{0}\right)\right]^{T}=} \\
& \underline{o}\left(\phi_{S}, \frac{\partial a}{\partial \lambda}\left(C_{S}\right), \frac{\partial^{2} a}{\partial \lambda \partial \phi}\left(C_{S}\right), \frac{\partial^{3} a}{\partial \lambda \partial \phi^{2}}\left(C_{S}\right), \phi_{N}, \frac{\partial a}{\partial \lambda}\left(C_{N}\right)\right. \\
& \left., \frac{\partial^{2} a}{\partial \lambda \partial \phi}\left(C_{N}\right), \frac{\partial^{3} a}{\partial \lambda \partial \phi^{2}}\left(C_{N}\right), \phi_{0}\right) \\
& {\left[\frac{\partial^{2} a}{\partial \lambda^{2}}\left(C_{0}\right) \frac{\partial^{3} a}{\partial \lambda^{2} \partial \phi}\left(C_{0}\right) \frac{\partial^{4} a}{\partial \lambda^{2} \partial \phi^{2}}\left(C_{0}\right)\right]^{T}=} \\
& \underline{o}\left(\phi_{S}, \frac{\partial^{2} a}{\partial \lambda^{2}}\left(C_{S}\right), \frac{\partial^{3} a}{\partial \lambda^{2} \partial \phi}\left(C_{S}\right), \frac{\partial^{4} a}{\partial \lambda^{2} \partial \phi^{2}}\left(C_{S}\right), \phi_{N}, \frac{\partial^{2} a}{\partial \lambda^{2}}\left(C_{N}\right)\right. \\
& \left., \frac{\partial^{3} a}{\partial \lambda^{2} \partial \phi}\left(C_{N}\right), \frac{\partial^{4} a}{\partial \lambda^{2} \partial \phi^{2}}\left(C_{N}\right), \phi_{0}\right)
\end{aligned}
$$

These calculations are facilitated by the fact that any one-dimensional quintic spline is completely defined by the function, first-derivative, and second-derivative values at the spline interval's two end points. Additional descriptions of bi-quintic splines that include many more details are available in Refs. [22] and [26]. 


\section{CHAPTER 3}

\section{BOUNCE POINTS, RAY-HOPS, AND RAY-PATHS}

\subsection{Definitions}

A bounce point is the geometric location of a traversing signal's incidence upon and reflection from the Earth. The position of the $k^{\text {th }}$ bounce point in Cartesian coordinates is denoted $\eta_{\mathrm{k}}$. The unit vector that is perpendicular to the Earth's surface at bounce point $k$ is called the bounce point normal vector and is denoted by $\underline{u}_{\mathrm{k}}$. The ray-path direction from which a signal approaches bounce point $k$ is $\underline{v}_{\mathrm{f}, \mathrm{k}}$. The direction of the reflected signal at bounce point $k$ is $\underline{v}_{0, \mathrm{k}}$.

The curved signal trajectory between the transmitter and the first bounce point, between two sequential bounce points, or between a bounce point and the receiver is regarded as a ray-hop. Ray-hops are denoted $s$. An ordered sequence of ray-hops that starts at a transmitter and ends at the location of the receiver, $\underline{r}_{\mathrm{R}}$, constitutes a ray-path. It should be noted that the possibility of multiple reflection-like refractions off of the ionosphere between a pair of reflections off the Earth, as described in Ref. [27], is not considered in the scope of this work.

Figure 4 illustrates these definitions, showing three sequential bounce points, the receiver location, the ray hops connecting them, and other terms. The associated vector $\hat{\underline{j}}_{\mathrm{j}}$ consists of all ionosphere parameters that apply in the vicinity of the $j^{\text {th }}$ ray-path that is illustrated in that figure. This means that once the position of all points along the signal's 
trajectory have been determined by a set of numerical raytracing computations, it is required to determine for each of those points the grid cell at which they lie. These points include points on the illustrated ray-hops $s_{\mathrm{k}}, s_{\mathrm{k}+1}, \ldots, s_{\mathrm{m}}$, along with additional ray-hops that belong to the ray-path that are not shown in Fig. 4. All $P_{x, x}^{\prime}$ and $P_{x, x}$ terms refer to range-equivalent group delays / beat carrier phases that will be considered in a later discussion.

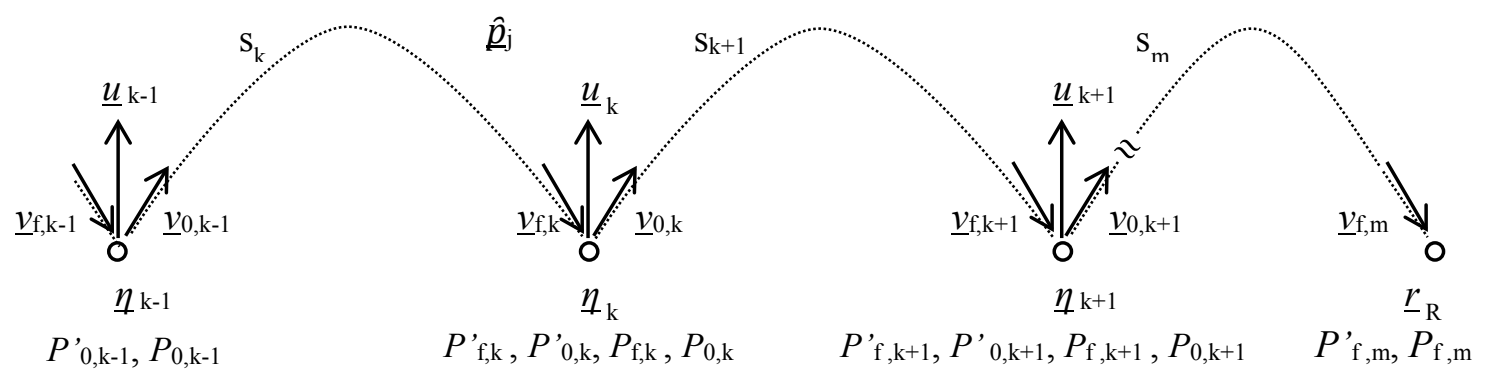

Figure 4: The ray-path definitions and notation.

\subsection{Bounce Points}

\subsubsection{Bounce point properties and representation}

In the scope of this study, it is assumed that waves are perfectly reflected from the Earth's surface in a specular reflection manner, although Ref. [27] suggests that ground reflections consist of superimposed specular and non-specular, randomly distributed components.

Reflectivity - the power of the reflected signal to that of the incident wave in an anisotropic medium is determined by Fresnel's equations as discussed in Ref. [28]. An 
additional important property of propagating signals that tends to change significantly during signal reflection is polarization. The current model does not account for the impact of reflectivity and the varying polarization. However, an extension for this study is planned to incorporate these effects.

Three equations are used to implicitly define each bounce point. The set of three equations that defines the $k^{\text {th }}$ bounce point of a given ray-path can be written in the following shorthand form

$$
\underline{0}=\underline{g}_{k}=\underline{g}_{k}\left(\underline{v}_{f, k}, \underline{v}_{0, k}, \underline{\eta_{k}}, \underline{u}_{k}\right)
$$

An explicit formulation of Eq. (29), split into three scalar components - components of types $\mathrm{A}, \mathrm{B}$, and $\mathrm{C}$ - takes the form

$$
\underline{0}=\underline{\underline{g}}_{k} \triangleq\left(\begin{array}{l}
g_{A, k}\left(\underline{v}_{f, k}, \underline{v}_{0, k}, \underline{\eta}_{k}, \underline{u}_{k}\right) \\
g_{B, k}\left(\underline{v}_{f, k}, \underline{v}_{0, k}, \underline{\eta}_{k}, \underline{u}_{k}\right) \\
g_{C, k}\left(\underline{v}_{f, k}, \underline{v}_{0, k}, \underline{\eta}_{k}, \underline{u}_{k}\right)
\end{array}\right)
$$

Recognizing that a signal's trajectory within a single ray hop, and in particular its directional vectors $\underline{v}_{0}$ and $\underline{v}_{f}$, depend on the location of the hop's start and end points and on the values taken by the ionosphere parameters that apply in the vicinity of that hop, Eq. (29) can be rewritten as

$$
\left\{\begin{array}{l}
\underline{0}=\underline{\hat{g}}_{k}\left(\underline{v}_{f, k}\left[\underline{\eta}_{k-1}, \underline{\eta}_{k}, \underline{\hat{p}}_{j}\right], \underline{v}_{0, k}\left[\underline{\eta}_{k}, \underline{\eta}_{k+1}, \underline{\hat{p}}_{j}\right], \underline{\eta}_{k}, \underline{u}_{k}\left[\underline{\eta}_{k}\right]\right), k<m-1 \\
\underline{0}=\underline{\hat{g}}_{k}\left(\underline{v}_{f, k}\left[\underline{\eta}_{k-1}, \underline{\eta}_{k}, \underline{\hat{p}}_{j}\right], \underline{v}_{0, k}\left[\underline{\eta}_{k}, \underline{r}_{R}, \underline{\hat{p}}_{j}\right], \underline{\eta}_{k}, \underline{u}_{k}\left[\underline{\eta}_{k}\right]\right), k=m-1
\end{array}\right.
$$


or, in a shorthand form

$$
\underline{0}=\underline{\tilde{g}}_{k}\left(\underline{\hat{\eta}}_{j}, \underline{r}_{R}, \underline{\hat{p}}_{j}\right)
$$

where $\hat{p}_{j}$ is as defined before, $m$ is the total number of ray-hops constituting the $j^{\text {th }}$ raypath, and where $\underline{\eta}_{\mathrm{j}}$ denotes the set of bounce points of the $j^{\text {th }}$ ray-path.

The formulation of Eqs. (31) and (32) is used with the ray-paths solver that is described in Section 3.4, as well as with the alternative batch filter of Chapter 5. The formulation of Eqs. (29) and (30) has been favored for most Gauss-Newton process related calculations for practical reasons that will become clear in the next subsection.

\subsubsection{Bounce point equations}

The $k^{\text {th }}$ Type-A constraint equation requires that the $k^{\text {th }}$ bounce point lies on the Earth surface. This surface is represented by the WGS-84 ellipsoid in the present study, although it should be represented by some sort of topographic map when dealing with real data.

Type-B constraint equations enforce co-planarity between the directional vector of the incoming ray-hop signal as it approaches the receiver, the directional vector of the reflected ray hop, and the normal vector to Earth's surface at the bounce point. For the $k^{\text {th }}$ bounce point, which links the $k^{\text {th }}$ ray hop and the $(k+1)^{\text {st }}$ ray hop, the following equation definition for $g_{B, k}$ applies:

$$
0=g_{B, k}\left(\underline{v}_{f, k}, \underline{v}_{0, k}, \underline{\hat{\eta}}_{j}, \underline{u}_{k}\right)=\underline{u}_{k} \cdot\left(\underline{v}_{f, k} \times \underline{v}_{0, k}\right)
$$


where $\underline{u}_{\mathrm{k}}$ is an outward unit vector normal to the Earth's surface at the $k^{\text {th }}$ bounce point. Let $h_{\mathrm{DTM}}\left(\phi_{0}, \lambda_{0}\right)$ be the scalar function that computes Earth surface's height above the WGS-84 ellipsoid at latitude $\phi_{0}$ and longitude $\lambda_{0}$. Let $\underline{r}_{\mathrm{ECEF}}\left(\phi_{0}, \lambda_{0}, h_{0}\right)$ be the function that computes Cartesian ECEF coordinates from geographic latitude/longitude/altitude coordinates. Define: $\underline{r}_{\mathrm{surf}}\left(\phi_{0}, \lambda_{0}\right)=\underline{r}_{\mathrm{ECEF}}\left(\phi_{0}, \lambda_{0}, h_{\mathrm{DTM}}\left[\phi_{0}, \lambda_{0}\right]\right)$ and note that $h_{\mathrm{DTM}}\left(\phi_{0}, \lambda_{0}\right)=0$ in the present study. Then $\underline{u}_{\mathrm{k}}$ is given by

$$
\underline{u}_{k}=\frac{\left(\left.\frac{\partial r_{\text {surf }}}{\partial \lambda}\right|_{\left(\phi_{0}, \lambda_{0}\right)}\right) \times\left(\left.\frac{\partial r_{\text {surf }}}{\partial \phi}\right|_{\left(\phi_{0}, \lambda_{0}\right)}\right)}{\left\|\left(\left.\frac{\partial r_{\text {surf }}}{\partial \lambda}\right|_{\left(\phi_{0}, \lambda_{0}\right)}\right) \times\left(\left.\frac{\partial r_{\text {surf }}}{\partial \phi}\right|_{\left(\phi_{0}, \lambda_{0}\right)}\right)\right\|}
$$

Type-C equations constrain the normal vector to the Earth at the bounce point to bisect the angle between the incoming and reflected ray hops. It can be written in the form

$$
0=g_{C, k}\left(\underline{v}_{f, k}, \underline{v}_{0, k}, \underline{\hat{\eta}}_{j}, \underline{u}_{k}\right)=\underline{u}_{k} \cdot\left(\left\|\underline{v}_{f, k}\right\| \underline{v}_{0, k}+\left\|\underline{v}_{0, k}\right\| \underline{v}_{f, k}\right)
$$

The + sign in this formula results from $\underline{v}_{f, k}$ pointing towards the reflection point while $\underline{v}_{0, k}$ points away from it. It should be noted that this formulation of the bounce-point conditions could potentially accept the case of an out-going ray-path direction equaling the exact negative of the in-coming ray-path direction, which is unacceptable. This can be addressed with a careful choice of first guesses to the ray-path-determination calculations, and by applying a feasibility test to computed pseudoranges. This test relies on the assumption that non-physically-feasible computed ray-paths will result in groupdelays that are significantly different from the measured group delays. 


\subsection{Single-Hop Calculations}

\subsubsection{The fundamental nonlinear two point boundary value problem}

Given the signal trajectory's known start and end location, and given a set of applicable ionosphere parameters, one can determine the ray-hop by determining the initial state $\underline{X}_{0}$ of the raytracing differential equation in Eq. (16) that applies at the beginning of the hop's trajectory and the total signal range-equivalent group delay $P_{\mathrm{f}}^{\text {' for }}$ which the signal ultimately arrives at the known end location. This Two-Point Boundary Value Problem (TPBVP) is thoroughly discussed in Ref. [16]. Therefore, only a brief description is given here. It should be noted that this study relies on the work that is described in that source.

Without loss of generality, the Hamiltonian at the beginning of the trajectory (and everywhere else along the ray's path) is assumed zero. The initial boundary conditions are therefore

$$
\left\{\begin{array}{l}
\underline{\eta}_{0}=P_{f}^{\prime}\left[\begin{array}{ll}
I & 0
\end{array}\right] \underline{X}_{0} \\
0=H\left(\underline{X}_{0}, \underline{\tilde{p}}\right)=H_{0}
\end{array}\right.
$$

and the final boundary condition is

$$
\underline{\eta}_{f}=P_{f}^{\prime}\left[\begin{array}{ll}
I & 0
\end{array}\right] \underline{X}_{f}
$$

where $\tilde{\underline{\alpha}}$ is a general vector of ionosphere model parameters that apply at a region of interest. $\eta_{0}$ and $\eta_{\mathrm{f}}$ denote a single-hop trajectory's start and end points, respectively. For instance, for ray-hop $s_{\mathrm{k}+1}$ that is shown in Fig. $4, \eta_{0}$ refers to $\eta_{\mathrm{k}}$ and $\eta_{\mathrm{f}}$ refers to $\eta_{\mathrm{k}+1}$. The 
notation $\underline{X}_{0}$ corresponds to the state vector at the beginning of a hop, and similarly, $\underline{X}_{f}$ corresponds to the value that the state vector takes at the end of a hop. This set of seven scalar equations is solved for the six elements of the state vector $\underline{X}_{0}$ and the final propagation range-equivalent group delay $P^{\prime}{ }_{\mathrm{f}}$. The solution algorithm involves calculating the sensitivity matrices $\partial \underline{X}_{\mathrm{f}} / \partial \underline{X}_{0}$ and $\partial \underline{X}_{\mathrm{f}} / \partial P^{\prime}{ }_{\mathrm{f}}$ which are required for the implementation of Newton's method in order to iteratively solve this nonlinear TPBVP. ${ }^{1}$

\subsubsection{Sensitivities to inputs}

As discussed earlier in the context of ray-path solution and in the broader perspective of the fundamental estimation problem, it is necessary to obtain the partial derivatives of the TPBVP problem with respect to some of its inputs. For the sake of simplicity, the concept for obtaining these sensitivities is demonstrated here with an unnormalized problem formulation as in Eqs. (9) and (16), with $P^{\prime}$ as the independent variable. An alternative implementation is presented in Ref. [16] that is slightly different, as it is aligned with a normalized formulation.

Let $\underline{\alpha}$ be $\underline{\tilde{X}} 0, \underline{\tilde{p}}$ or $P^{\prime}$, then by differentiating Eq. (16) with respect to $\underline{\alpha}$ :

${ }^{1}$ Subsections 3.3.1 - 3.3.5 are based on the information provided in the electronically submitted supplementary file notes_on_sensitivities.pdf, with slight modifications and some notational adaptation. 


$$
\begin{aligned}
& \frac{\partial}{\partial \underline{\alpha}}\left(\frac{d \underline{\tilde{X}}}{d P^{\prime}}\right)=\frac{\partial}{\partial \underline{\alpha}}(\underline{\tilde{f}}) \\
& \downarrow \\
& \frac{d}{d P^{\prime}}\left(\frac{\partial \underline{\tilde{X}}}{\partial \underline{\alpha}}\right)=\left.\frac{\partial \tilde{\tilde{f}}}{\partial \underline{\tilde{X}}}\right|_{\underline{\tilde{X}}_{P^{\prime}}, \underline{\tilde{p}}} \frac{\partial \underline{\tilde{X}}}{\partial \underline{\alpha}}+\left.\frac{\partial \tilde{f}}{\partial \underline{\tilde{p}}}\right|_{\tilde{\tilde{X}}_{P^{\prime}}, \underline{\tilde{p}}} \frac{\partial \underline{\tilde{p}}}{\partial \underline{\alpha}}
\end{aligned}
$$

This last equation is of immense importance, as it enables relating partial derivatives of $\underline{\tilde{X}}$ with respect to various parameters at different times through numerical integration. This equation reduces to the familiar linearized state-transition matrix differential equation in the case where $\underline{\alpha}$ is $\underline{\tilde{X}}_{0}$. It is a sensitivity matrix or vector equation if $\underline{\alpha}$ is, respectively, $\underline{\tilde{p}}$ or $P_{\text {f. }}^{\prime}$.

\subsubsection{Computation of $\partial \underline{v}_{\mathrm{j}} / \partial \underline{\tilde{p}}$ and $\partial \underline{\underline{v}}_{0_{j-1}} / \partial \underline{\tilde{p}}$}

The short notation that utilizes subscripts 0 and $f$ is used here as before. For the sake of simplicity, the hop index notation is omitted for the rest of this subsection as this subsection only considers a single ray-hop - $s_{\mathrm{j}}$, and so $\underline{v}_{0} \equiv \underline{v}_{\mathrm{j}-1}, \underline{v}_{\mathrm{f}} \equiv \underline{\mathrm{v}}_{\mathrm{j}}, P^{\prime}{ }_{o} \equiv P^{\prime}{ }_{o_{j-1}}$ and $P_{f}^{\prime} \equiv P_{f_{j}}^{\prime}$. The same compact notation will be used for the vectors $\underline{r}, \underline{k}$ and $\underline{\tilde{X}}$. It should be noted that $\partial \underline{v}_{0}{ }_{\mathrm{j}-1} / \partial \tilde{\tilde{p}}$ is the same as $\partial \underline{v}_{0} / \partial \tilde{\tilde{p}}$ or $\left[0,1 / k_{0} \cdot \mathrm{I}\right]\left(\partial \underline{\tilde{X}}_{0} / \partial \underline{\tilde{p}}\right)$ evaluated at the beginning of the $j^{\text {th }}$ hop, i.e. at $P^{\prime}{ }_{o_{j-1}}$. Similarly, $\partial \underline{v}_{\mathrm{j}} / \partial \tilde{\tilde{p}}$ is the same as $\partial \underline{v}_{\mathrm{f}} / \partial \underline{\tilde{p}}$ or $\left[0,1 / k_{0} \cdot \mathrm{I}\right]\left(\partial \underline{\tilde{X}}_{\mathrm{f}} / \partial \underline{\tilde{p}}\right)$ evaluated at the end of the $j^{\text {th }}$ hop, i.e. at $P_{f_{j}}^{\prime}$. The last statement is true since all bounce points are located well below the ionosphere where the wave number takes on its nominal value: $k=k_{0}$.

For this computation, the initial and final locations of the signal's trajectory are assumed known and fixed. From Eq. (38) with $\underline{\alpha} \equiv \underline{\tilde{p}}$, a $P^{\prime}$-dependent ODE is obtained: 


$$
\frac{d}{d P^{\prime}}\left(\frac{\partial \underline{\tilde{X}}}{\partial \underline{\tilde{p}}}\right)=\left.\frac{\partial \tilde{f}}{\partial \underline{\tilde{X}}}\right|_{\underline{\tilde{X}}_{p}, \underline{\underline{p}}} \frac{\partial \underline{\tilde{X}}}{\partial \underline{\tilde{p}}}+\left.\frac{\partial \tilde{f}}{\partial \underline{\tilde{p}}}\right|_{\underline{\tilde{x}}_{P}, \underline{\underline{p}}}
$$

The first set of boundary condition equations considers the known location of the trajectory's start point which is independent of ionospheric parameters

$$
0=\left.\left[\begin{array}{ll}
I & 0
\end{array}\right] \frac{\partial \underline{\tilde{X}}}{\partial \underline{\tilde{p}}}\right|_{P_{0}^{\prime}}=\left[\begin{array}{ll}
I & 0
\end{array}\right] \frac{\partial \underline{X}_{0}}{\partial \underline{\tilde{p}}}
$$

This yields $3 \times N_{\mathrm{p}}$ equations where $N_{\mathrm{p}}$ is the number of ionosphere parameters in $\tilde{\underline{p}}$. An additional set of $N_{\mathrm{p}}$ equations derives from the independence of the Hamiltonian initial (and constant) value from the ionosphere parameters:

$$
0=\left.\frac{d H}{d \underline{\tilde{p}}}\right|_{P_{0}^{\prime}}=\left.\left.\frac{\partial H}{\partial \underline{k}}\right|_{P_{0}^{\prime}}\left[\begin{array}{ll}
0 & I
\end{array}\right] \frac{\partial \underline{\tilde{X}}}{\partial \underline{\tilde{p}}}\right|_{P_{0}^{\prime}}+\left.\frac{\partial H}{\partial \underline{\tilde{p}}}\right|_{P_{0}^{\prime}}=\left.\frac{\partial H}{\partial \underline{k}}\right|_{P_{0}^{\prime}}\left[\begin{array}{ll}
0 & I
\end{array}\right] \frac{\partial \underline{\tilde{X}_{0}}}{\partial \underline{\tilde{p}}}+\frac{\partial H_{0}}{\partial \underline{\tilde{p}}}
$$

The final boundary condition is

$$
\begin{aligned}
0=\left.\frac{d \underline{r}_{w}}{d \underline{\tilde{p}}}\right|_{P_{f}^{\prime}} & =\left[\begin{array}{ll}
I & 0
\end{array}\right]\left(\left.\frac{\partial \underline{\tilde{X}}}{\partial \underline{\tilde{p}}}\right|_{P_{f}^{\prime}}+\left.\frac{d \underline{\tilde{X}}}{d P^{\prime}}\right|_{P_{f}^{\prime}} \frac{\partial P_{f}^{\prime}}{\partial \underline{\tilde{p}}}\right) \\
& =\left[\begin{array}{ll}
I & 0
\end{array}\right]\left(\frac{\partial \underline{\tilde{X}}_{f}}{\partial \underline{\tilde{p}}}+\frac{d \underline{\tilde{X}}_{f}}{d P_{f}^{\prime}} \frac{\partial P_{f}^{\prime}}{\partial \underline{\tilde{p}}}\right)
\end{aligned}
$$

This is a set of $3 \times N_{\mathrm{p}}$ equations, bringing the total number of equations to $7 \mathrm{x} N_{\mathrm{p}}$. The equations are solved for $\partial \underline{\underline{X}}_{0} / \partial \underline{\tilde{q}}\left(6 \mathrm{x} N_{\mathrm{p}}\right.$ unknowns) and $\partial P_{\mathrm{f}}^{\prime} / \partial \underline{\underline{\underline{x}}}$ ( $N_{\mathrm{p}}$ unknowns). The matrices $\partial \underline{\tilde{X}}_{f} / \partial \tilde{\underline{p}}$ and $\partial \underline{\underline{X}}_{f} / \partial P^{\prime}{ }_{\mathrm{f}}$ are related to their values at $P^{\prime}{ }_{0}$ through dynamic propagation of the state influence matrix differential Eq. (39). It should also be noted that: 


$$
\begin{aligned}
& \underline{v}_{0}=\left[\begin{array}{ll}
I & 0
\end{array}\right] \underline{\tilde{f}}\left(\underline{\tilde{X}}_{0}, \underline{\tilde{p}}\right) \\
& \underline{v}_{f}=\left[\begin{array}{ll}
I & 0
\end{array}\right] \underline{\tilde{f}}\left(\underline{\tilde{X}}_{f}, \underline{\tilde{p}}\right)
\end{aligned}
$$

Then the full derivative of $\underline{v}_{0}$ with respect to $\tilde{\underline{\alpha}}$ is

$$
\frac{d \underline{v}_{0}}{d \underline{\tilde{p}}}=\left[\begin{array}{ll}
I & 0
\end{array}\right]\left[\left.\frac{\partial \overline{\tilde{f}}}{\partial \underline{\tilde{X}}}\right|_{\underline{\tilde{X}}_{0}, \underline{\underline{p}}} \frac{\partial \underline{\tilde{X}}}{\partial \underline{\tilde{p}}}+\left.\frac{\partial \tilde{\tilde{f}}}{\partial \underline{\tilde{p}}}\right|_{\underline{\tilde{X}}_{0}, \underline{\underline{\underline{p}}}}\right]
$$

and for $\underline{\mathrm{f}}$ :

$$
\begin{aligned}
& \frac{d \underline{\underline{v}}_{f}}{d \underline{\tilde{p}}}=\left[\begin{array}{ll}
I & 0
\end{array}\right]\left[\left.\frac{\partial \underline{\tilde{f}}}{\partial \underline{\tilde{X}}}\right|_{\underline{\tilde{x}}_{f}, \underline{\underline{\underline{p}}}}\left(\frac{\partial \underline{\tilde{X}}_{f}}{\partial \underline{\tilde{X}}_{0}} \frac{\partial \underline{\tilde{X}}_{0}}{\partial \underline{\tilde{p}}}+\frac{\partial \underline{\tilde{X}}_{f}}{\partial P_{f}^{\prime}} \frac{\partial P_{f}^{\prime}}{\partial \underline{\tilde{p}}}\right)+\left.\frac{\partial \tilde{f}}{\partial \underline{\tilde{\tilde{p}}}}\right|_{\underline{\tilde{X}}_{f}, \underline{\tilde{p}}}\right] \\
& =\left[\begin{array}{ll}
I & 0
\end{array}\right]\left[\left.\frac{\partial \tilde{\tilde{f}}}{\partial \underline{\tilde{X}}}\right|_{\underline{\tilde{X}}_{f}, \underline{\tilde{p}}}\left(\frac{\partial \underline{\tilde{X}}_{f}}{\partial \underline{\tilde{X}}_{0}} \frac{\partial \underline{\tilde{X}}_{0}}{\partial \underline{\tilde{p}}}+\underline{\tilde{f}}\left(\underline{\tilde{X}}_{f}, \underline{\tilde{p}}\right) \frac{\partial P_{f}^{\prime}}{\partial \underline{\tilde{p}}}\right)+\left.\frac{\partial \tilde{\tilde{f}}}{\partial \underline{\tilde{p}}}\right|_{\underline{\tilde{X}}_{f}, \underline{\tilde{p}}}\right]
\end{aligned}
$$

where the solution for the two unknowns $\partial \underline{\underline{X}}_{0} / \partial \tilde{\underline{\underline{p}}}$ and $\partial P^{\prime} f / \partial \tilde{\underline{p}}$ that was obtained from Eqs. (41) and (42) is substituted in, and the two partial derivatives of $\underline{\tilde{X}}$ that are evaluated at $P_{\mathrm{f}}^{\prime}$ are obtained through propagation of the state transition matrix equation.

\subsubsection{Computation of $\partial \underline{\underline{v}}_{\mathrm{j}} / \partial \underline{\eta}_{0}$ and $\partial \underline{\underline{v}}_{\mathrm{j}_{\mathrm{j}-1}} / \partial \underline{\eta}_{0}$}

In this subsection sensitivities of the direction of the wave vector at the signal's start and end points to variations in the initial ray-hop location are computed. The ionosphere parameters set $\tilde{\underline{p}}$ is consequently assumed fixed.

Eq. (38) takes the form 


$$
\left.\frac{d}{d P^{\prime}}\left(\frac{\partial \underline{\tilde{X}}}{\partial \underline{\eta}_{0}}\right)\right|_{P^{\prime}}=\left.\left.\frac{\partial f}{\partial \underline{\tilde{X}}}\right|_{\underline{\tilde{X}}_{p}, \tilde{\underline{\underline{p}}}} \frac{\partial \underline{\tilde{X}}}{\partial \underline{\eta}_{0}}\right|_{\underline{\tilde{X}}_{p},, \underline{\underline{\underline{p}}}}
$$

The first initial boundary condition is straightforward and yields three equations

$$
I=\left.\left[\begin{array}{ll}
I & 0
\end{array}\right] \frac{\partial \underline{\tilde{X}}}{\partial \underline{\eta}_{0}}\right|_{P_{0}^{\prime}}=\left[\begin{array}{ll}
I & 0
\end{array}\right] \frac{\partial \underline{\tilde{X}}_{0}}{\partial \underline{\eta}_{0}}
$$

The second initial boundary condition derives from the independence of the Hamiltonian and the initial location of the hop and it takes the form (the term in brackets is a $1 \times 6$ vector)

$$
0=\left[\left.\left.\frac{\partial H}{\partial \underline{r}_{w}}\right|_{\underline{\tilde{X}}_{0}, \underline{\tilde{p}}} \frac{\partial H}{\partial \underline{k}}\right|_{\underline{\tilde{X}}_{0}, \underline{\underline{p}}}\right] \frac{\partial \underline{\tilde{X}}_{0}}{\partial \underline{\eta_{0}}}
$$

For the final boundary condition, the independence of the signal's front position at the start and end times is considered

$$
0=\left.\frac{d \underline{r}_{w}}{d \underline{\eta}_{0}}\right|_{P_{f}^{\prime}}=\left[\begin{array}{ll}
I & 0
\end{array}\right]\left(\left.\frac{\partial \underline{\tilde{X}}}{\partial \underline{\eta_{0}}}\right|_{P_{f}^{\prime}}+\left.\frac{d \underline{\tilde{X}}}{d P^{\prime}}\right|_{P_{f}^{\prime}} \frac{\partial P_{f}^{\prime}}{\partial \underline{\eta}_{0}}\right)=\left[\begin{array}{ll}
I & 0
\end{array}\right]\left(\frac{\partial \underline{\tilde{X}}_{f}}{\partial \underline{\eta}_{0}}+\frac{d \underline{\tilde{X}}_{f}}{d P_{f}^{\prime}} \frac{\partial P_{f}^{\prime}}{\partial \underline{\eta}_{0}}\right)
$$

Eqs. (47), (48) and (49) can be solved for $\partial \underline{\tilde{X}}_{0} / \partial \underline{\Pi}_{0}$ and $\partial P^{\prime}{ }_{\mathrm{f}} / \partial \underline{\Pi}_{0}$ where once again the sensitivities at different times can be related by numerically integrating the ODE for sensitivities. The two quantities can be substituted back into the $\underline{v}_{0}$ and $\underline{v}_{\mathrm{f}}$ partial derivative formulas to obtain the full derivatives of interest: 


$$
\begin{aligned}
& \frac{d \underline{v}_{0}}{d \underline{\eta}_{0}}=\left[\begin{array}{ll}
I & 0
\end{array}\right]\left[\left.\frac{\partial \tilde{f}}{\partial \underline{\tilde{X}}}\right|_{\underline{\tilde{X}}_{0}, \underline{\tilde{p}}} \frac{\partial \underline{\tilde{X}}_{0}}{\partial \underline{\eta}_{0}} \mid\right] \\
& \frac{d \underline{v}_{f}}{d \underline{\eta}_{0}}=\left[\begin{array}{ll}
I & 0
\end{array}\right]\left[\left.\frac{\partial \tilde{f}}{\partial \underline{\tilde{X}}}\right|_{\underline{X}_{0}, \underline{\tilde{p}}}\left\{\frac{\partial \underline{\tilde{X}}_{f}}{\partial \underline{\eta}_{0}}+\underline{f}\left(\underline{\tilde{X}}_{f}, \underline{\tilde{p}}\right) \frac{\partial P_{f}^{\prime}}{\partial \underline{\eta}_{0}}\right\}\right]
\end{aligned}
$$

\subsubsection{Computation of $\partial \underline{v}_{f_{\mathrm{j}}} / \partial \underline{\eta}_{\mathrm{f}}$ and $\partial \underline{v}_{0_{\mathrm{j}-1}} / \partial \underline{\eta}_{\mathrm{f}}$}

Similar to the previous derivation:

$$
\left.\frac{d}{d P^{\prime}}\left(\frac{\partial \underline{\tilde{X}}}{\partial \underline{\eta_{f}}}\right)\right|_{P^{\prime}}=\left.\frac{\partial \tilde{\tilde{f}}}{\partial \underline{\tilde{X}}}\right|_{\underline{\tilde{X}}_{P}, \underline{\tilde{p}}} \frac{\partial \underline{\tilde{X}}}{\partial \underline{\eta}_{f}}
$$

The first initial boundary condition is straightforward and yields three equations

$$
0=\left.\left[\begin{array}{ll}
I & 0
\end{array}\right] \frac{\partial \underline{\tilde{X}}}{\partial \underline{\eta}_{f}}\right|_{P_{0}^{\prime}}=\left[\begin{array}{ll}
I & 0
\end{array}\right] \frac{\partial \underline{\tilde{X}}_{0}}{\partial \underline{\eta}_{f}}
$$

The second initial boundary condition derives from the independence of the Hamiltonian and the final location of the hop and it takes the form (the term in brackets is a $1 \times 6$ vector)

$$
0=\left[\left.\left.\frac{\partial H}{\partial \underline{r}_{w}}\right|_{\underline{\tilde{X}}_{0}, \tilde{\underline{p}}} \frac{\partial H}{\partial \underline{k}}\right|_{\underline{\tilde{x}}_{0}, \underline{\tilde{p}}}\right] \frac{\partial \underline{\tilde{X}}_{0}}{\partial \underline{\eta}_{f}}
$$

For the final boundary condition, the obvious coinciding of $\underline{r}_{\mathrm{w}}$ and $\underline{\eta}_{\mathrm{f}}$ is considered 


$$
I=\left.\frac{d \underline{r}_{w}}{d \underline{\eta}_{f}}\right|_{P_{f}^{\prime}}=\left[\begin{array}{ll}
I & 0
\end{array}\right]\left(\left.\frac{\partial \underline{\tilde{X}}}{\partial \underline{\eta_{f}}}\right|_{P_{f}^{\prime}}+\left.\frac{d \underline{\tilde{X}}}{d P^{\prime}}\right|_{P_{f}^{\prime}} \frac{\partial P_{f}^{\prime}}{\partial \underline{\eta}_{f}}\right)=\left[\begin{array}{ll}
I & 0
\end{array}\right]\left(\frac{\partial \underline{\tilde{X}}_{f}}{\partial \underline{\eta}_{f}}+\frac{d \underline{\tilde{X}}_{f}}{d P_{f}^{\prime}} \frac{\partial P_{f}^{\prime}}{\partial \underline{\eta}_{f}}\right)
$$

The last three equations can be solved for $\partial \underline{\underline{X}}_{0} / \partial \underline{\eta}_{\mathrm{f}}$ and $\partial P^{\prime}{ }_{\mathrm{f}} / \partial \underline{\underline{f}}_{\mathrm{f}}$ with the sensitivities at different times related through numerically integrating the ODE for sensitivities. Finally, the two quantities can be substituted back into the $\underline{v}_{0}$ and $\underline{v}_{\mathrm{f}}$ partial derivative formulas to obtain the full derivatives:

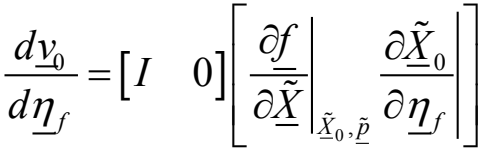

$$
\begin{aligned}
& \frac{d \underline{v}_{f}}{d \underline{\eta}_{f}}=\left[\begin{array}{ll}
I & 0
\end{array}\right]\left[\left.\frac{\partial \underline{f}}{\partial \underline{\tilde{X}}}\right|_{\underline{\tilde{x}}_{f}, \underline{\tilde{\tilde{p}}}}\left(\frac{\partial \underline{\tilde{X}}_{f}}{\partial \underline{\eta}_{f}}+\underline{f}\left(\underline{\tilde{X}}_{f}, \underline{\tilde{p}}\right) \frac{\partial P_{f}^{\prime}}{\partial \underline{\eta}_{f}}\right)\right]
\end{aligned}
$$




\subsection{Multiple-Hop Calculations: The Ray-Path Solver}

The work that is presented in this section utilizes the products of the past effort of Ref. [16] that was reviewed in the previous section. In the following discussion, single-hop calculations are extended to multiple-hop calculations that include determination of bounce points locations and their sensitivities to parameters, group delay and rangeequivalent beat carrier phase measurement models, and measurements' sensitivities to input parameters.

\subsubsection{Solving for the bounce points $\underline{\eta}$}

Determination of the ray-path for an HF signal that is traversing from a transmitter beacon to a receiver involves solution of coupled, nonlinear equations that define the physical characteristics of its trajectory. Suppose that one is given the following information: the locations of the receiver and the transmitter, the number of ray-path hops connecting them, and a model for the ionosphere. The goal of this section is to solve for the position of the ground bounce points that satisfy the governing reflection equations. In other words, this subsection develops a means of solving for the vector $\underline{\eta}$ as a function of the transmitter location, the receiver location, and the ionosphere model.

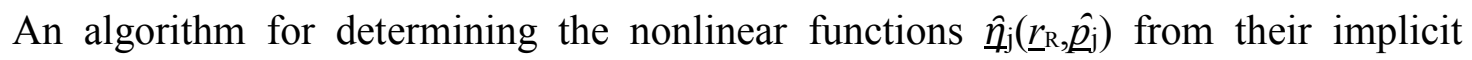
equations, or simply - a ray-path solver, has been developed. The ray-path solver assumes fixed known locations for the signal's start and end points, fixed ionosphere parameters, and a known number of sequenced ray-hops that constitute the ray-path. The ray-path solver's outputs are the locations of the bounce points. Auxiliary outputs are the partial derivatives of these locations with respect to the ionosphere parameters and their 
partial derivatives with respect to the location of the ray-path's end point. An additional output is a set of directional vectors for the incoming and reflected signals at the locations of the transmitter, the bounce points, and the receiver.

The solution is obtained using Newton's iterative method to solve:

$$
\underline{0}=\underline{\tilde{g}}_{m}\left(\underline{\hat{\eta}}_{j}, \underline{r}_{R}, \underline{\hat{p}}_{j}\right)
$$

through a minimization of

$$
J_{R P}\left(\underline{\hat{\eta}}_{j}\right)=\frac{1}{2} \underline{\tilde{g}}_{m}\left(\underline{\bar{\eta}}_{j}, \underline{r}_{R}, \underline{\hat{p}}_{j}\right)^{T} \underline{\tilde{g}}_{m}\left(\underline{\bar{\eta}}_{j}, \underline{r}_{R}, \underline{\hat{p}}_{j}\right)
$$

with respect to $\hat{\eta}_{\mathrm{j}}$. Here, $\tilde{g}_{\mathrm{m}}$ denotes the Eq. (32)-type formulation of the set of $3 \mathrm{x} m$ equations that apply at the $m$ bounce points of ray-path $j$ that has $m+1$ connected hops. $\hat{\eta}_{\mathrm{j}}$ is the stacked vector of $3 \mathrm{xm}$ coordinates of the ray-path's bounce points. The iterative solution process uses linearization about a current guess to compute a solution increment. It takes a step along the resulting search direction with a step-length scaling in the $\hat{\eta}_{\mathrm{j}}$ space that is chosen to ensure that the Eq. (58) new cost at the new guess of the solution is lower than the cost at the previous guess. A line search is essential in order to guarantee a decrease in cost and convergence to a solution. It starts by determining the Newton step through linearization of Eq. (57) with respect $\hat{\underline{y}}_{\mathrm{j}}$, where all terms are evaluated at a current guess $\hat{\eta}_{\mathrm{j}}$,guess

$$
\left.\frac{D \underline{\tilde{g}_{m}}}{D \underline{\hat{\eta}}_{j}}\right|_{\underline{\eta}_{j, \text { guess }}} \Delta \underline{\hat{\eta}}_{j}=-\underline{\tilde{g}}_{m}\left(\underline{\hat{\eta}}_{j, \text { guess }}, \underline{r}_{R}, \underline{\hat{p}}_{j}\right)
$$


and where $D$ denotes the total derivative operator. The solution for $\Delta \hat{\underline{q}}_{\mathrm{j}, \mathrm{g}}$, that constitutes the Gauss-Newton step (or correction vector), is computed by matrix inversion. The cost function of Eq. (58) is evaluated next with a series of improved guesses for $\hat{\eta}_{\mathrm{j}}$ that are obtained using

$$
\underline{\underline{\eta}}_{j, i}=\underline{\eta}_{j}+\alpha_{i} \cdot \Delta \underline{\eta}_{j, \text { guess }} \quad ; \quad \alpha_{i}=0.5^{i} \quad i=0,1,2,3, \ldots
$$

The new guess for $\hat{\eta}_{\mathrm{j}}, \underline{\underline{\eta}}_{\mathrm{j}, \text { new }}$, equals the value of $\hat{\underline{\eta}}_{\mathrm{j}, \mathrm{i}}$ for the smallest value of $i$ that yields a reduction in the cost function.

For bounce point $k$, the required set of sensitivities that are included in the left most term of Eq. (59) is obtained through computation of the total derivative

$$
\frac{D \underline{\tilde{g}}_{k}}{D \underline{\eta}_{l}}=\frac{\partial \underline{\tilde{g}}_{k}}{\partial \underline{v}_{f, k}} \frac{\partial \underline{v}_{f, k}}{\partial \underline{\eta}_{l}}+\frac{\partial \underline{\tilde{g}}_{k}}{\partial \underline{v}_{0, k}} \frac{\partial \underline{v}_{0, k}}{\partial \underline{\eta}_{l}}+\frac{\partial \underline{\tilde{g}}_{k}}{\partial \underline{\eta}_{l}}
$$

where $\tilde{g}_{k}$ is the subset of the elements of $\tilde{g}_{\mathrm{m}}$, consisting of the three equations that apply at bounce point $\underline{\eta}_{k}$, and where $\underline{\eta}_{l}$ is the $l^{\text {th }}$ bounce point of $\underline{\underline{j}}_{\mathrm{j}} \cdot \underline{v}_{\mathrm{f}, \mathrm{k}}$ is the direction of the signal that is arriving at point $\underline{\eta}_{\mathrm{k}}$, and $\underline{v}_{0, \mathrm{k}}$ is the direction of the reflected signal that is bouncing off at that point. The complete set of sensitivities $D \tilde{g}_{\mathrm{m}} / D \hat{\underline{\eta}}_{\mathrm{j}}$ is obtained by performing the above calculation for all bounce points of that ray-path.

Computations of $\partial \tilde{g}_{\mathrm{k}} / \partial \underline{v}_{\mathrm{f}, \mathrm{k}}, \partial \tilde{g}_{\mathrm{k}} / \partial \underline{v}_{\mathrm{f}, \mathrm{k}}$ and $\partial \tilde{g}_{\mathrm{k}} / \partial \underline{\eta}_{\mathrm{k}}$ are analytical and therefore immediate, as noted earlier. However, computation of $\partial \underline{v_{\mathrm{f}, \mathrm{k}}} / \partial \underline{\eta}_{\mathrm{k}-1}, \partial \underline{v}_{\mathrm{f}, \mathrm{k}} / \partial \underline{\eta}_{\mathrm{k}}, \partial \underline{v}_{0, \mathrm{k}} / \partial \underline{\eta}_{\mathrm{k}}$ and $\partial \underline{v}_{0, \mathrm{k}} / \partial \underline{\eta}_{\mathrm{k}+1}$ is implemented as an auxiliary of numerical raytracing, as described in the previous section. Note that these are the only $\underline{\eta}_{1}$ partial derivatives of $\underline{v}_{\mathrm{f}, \mathrm{k}}$ and $\underline{v}_{0, \mathrm{k}}$ that are non-zero in Eq. (61). 


\subsubsection{Ray-path solver robustness}

In order to maximize reliability and robustness of the ray-path solver, the algorithm attempts to solve the minimization problem with several sets of initial guesses. Priority is given to initial guesses that are input to the algorithm. The input of an initial guess is optional. An initial guess might be input in the situation where the ray-path solution is known to be close to a previously determined solution. When such a solution is not available, the algorithm will use the output of a ray-path solver that is based on a simplified ionosphere model as its first guess. This simplified solver has been used with earlier work that has been described in Ref. [5] as its primary ray-path solver. It has been observed that it yields an approximate solution for $\underline{\eta}$ that is typically close to the actual solution, and therefore, it is beneficial to use that solution as an initial guess for the fullmodel solver. Furthermore, it has been shown that the ray-hop segments that are obtained with the simplified solver can be successfully used as initial guesses for the direction of both incoming and reflected signals. This simple solver is discussed in Chapter 5.

Although uncommon, in some cases, use of the approximate solution as the first guess will fail to yield convergence, making it necessary to obtain additional initial guesses. A third type of initial guess is generated based on a thin-shell, latitude/longitude height dependent ionosphere model. The height for this bi-quintic spline modeled thin-shell is the height for which electron density reaches $80 \%$ of its maximal density. This method is also discussed briefly in Chapter 5. A fourth type of initial guess is generated using a constant-height thin-shell ionosphere model, with various heights considered. 


\subsubsection{Ray-paths' setup feasibility, solution existence and uniqueness}

Every ray-path setup is evaluated for physical feasibility. With simulated test cases, this evaluation is performed by trying to compute a raytracing solution using the true ionosphere model. The answer for the feasibility question is often not straightforward as it consists of two complementary questions: a) Does a solution for the considered setup exist? b) Can the solution be found with the ray-path solver? In the absence of an ability to distinguish between a negative answer for (a) and a negative answer for (b) during this phase of assessment, failures in obtaining a solution for $\underline{\eta}$ during this stage of assessment are regarded as an indication for the setup being physically unfeasible. This approach may raise the question of how many of the ray-paths that were declared unfeasible are in fact feasible, meaning that an incorrect determination originated from a ray-path solver failure. This is hard to determine given that the ray-path solver is currently the only tool available for feasibility assessment. However, based on comparison between results obtained with the current ray-path solver and results obtained with the simplified model ray-path solver that was used in the work of Ref. [5] for identical setups, the author tends to believe that most declared failures are due to physical infeasibility.

Ray-path solution uniqueness is a second matter that should be addressed. Multiple solutions are theoretically possible if the cost function (58) has multiple minima that are zero, as demonstrated in Ref. [29].

In the early work that utilized a simplified ray-path model, a given set of parameters that includes transmitter and receiver position, an ionosphere model, and the number of ray-hops sometimes yielded more than one possible solution. Such observations have not 
been made so far with the full, raytracing based model. This could be attributed to either genuine uniqueness of solution with the advanced model, or to the fact that the set of initial guesses for a given setup consists of vectors that are typically close to each other and converge to the same solution because of their closeness. The current algorithm, meanwhile, does not consider the possibility of having more than one solution for $\eta$.

A future version of the algorithm may need to possess the capability to address such ambiguities. A possible strategy might rely on tests where ray-path solutions are assessed through a comparison between the magnitudes of several ray-paths' residual terms (i.e., the differences between computed and measured group delays). Such a test is described later where a modified Gauss-Newton method is presented. 


\subsection{Measurement Models}

\subsubsection{The group delay measurement model}

A typical signal runs from the transmitter, traverses through the ionosphere in a refraction-based curved trajectory, bounces off of Earth and eventually arrives at the receiver. Let $\rho_{g, j}=P_{\mathrm{f}, \mathrm{m}(j)}^{\prime}$ be the true total range-equivalent group delay of the $j^{\text {th }}$ ray-path, which equals the true signal propagation time multiplied by the speed of light $c$. Let $y_{g, j}$ be the measured range-equivalent group delay of that ray-path, which equals the speed of light multiplied by the difference between the measured reception time according to the erroneous receiver clock and the true transmission time according to a calibrated beacon transmitter clock. Let $\delta$ be the receiver clock's offset. Then the $j^{\text {th }}$ group delay measurement equation can be written as

$$
\begin{aligned}
y_{g, j} & =h_{g, j}\left(\underline{x}_{g}, \underline{\hat{p}}_{j}\right)+v_{g, j}=\breve{h}_{g, j}\left(\underline{r}_{R}, \underline{\hat{p}}_{j}\right)+c \delta+v_{g, j} \\
& =\tilde{h}_{g, j}\left[\underline{r}_{R}, \underline{\eta}_{m}\left(\underline{r}_{R}, \underline{\hat{p}}_{j}\right), \underline{\hat{p}}_{j}\right]+c \delta+v_{g, j}
\end{aligned}
$$

where

$$
\underline{x}_{g} \triangleq\left[\begin{array}{c}
r_{R} \\
c \delta
\end{array}\right]
$$

is the vector of unknown receiver position components and the range-equivalent clock-

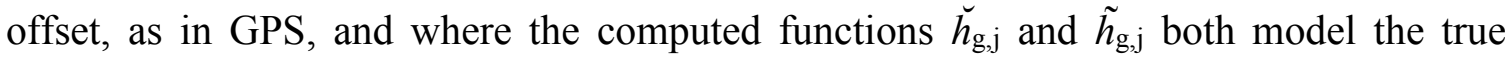
range-equivalent group delay of the $j^{\text {th }}$ ray-path $-\rho_{g, j}$. The vector $\hat{p}_{j}$ contains the ionosphere model parameters that apply in the vicinity of that ray-path, and $v_{\mathrm{g}, \mathrm{j}}$ is the measurement noise term. $\underline{\eta}_{m}$ is an explicit function representation of the elements of $\hat{\eta}_{j}$ 
that contains the ordered set of ECEF X-Y-Z coordinate functions of the $j^{\text {th }}$ ray-path's $m$ 1 unknown bounce points in the following form:

$$
\begin{gathered}
\underline{\eta}_{m}\left(\underline{r}_{R}, \underline{\hat{p}}_{j}\right)=\left[X_{1}\left(\underline{r}_{R}, \underline{\hat{p}}_{j}\right), X_{2}\left(\underline{r}_{R}, \underline{\hat{p}}_{j}\right), \ldots, X_{m-1}\left(\underline{r}_{R}, \underline{\hat{p}}_{j}\right), Y_{1}\left(\underline{r}_{R}, \underline{\hat{p}}_{j}\right), Y_{2}\left(\underline{r}_{R}, \underline{\hat{p}}_{j}\right), \ldots\right. \\
\left., Y_{m-1}\left(\underline{r}_{R}, \underline{\hat{p}}_{j}\right), Z_{1}\left(\underline{r}_{R}, \underline{\hat{p}}_{j}\right), Z_{2}\left(\underline{r}_{R}, \underline{\hat{p}}_{j}\right), \ldots, Z_{m-1}\left(\underline{r}_{R}, \underline{\hat{p}}_{j}\right)\right]
\end{gathered}
$$

The above non-linear functions use the geometric location for the receiver and the local ionosphere model to calculate the scalar pseudorange value.

The measurement model in Eq. (62) applies for a total of $N$ measured pseudoranges in a given navigation/ionosphere-correction problem. For convenience in batch estimation, this model is stacked into an $\mathrm{N}$-dimensional vector equation model of all the measurements. Let $\underline{\hat{q}}$ equal the union of all $\hat{\underline{j}}_{\mathrm{j}}$ vectors applying for all $N$ ray-paths. The stacked measurement model vector equation takes the form

$$
\left[\begin{array}{c}
y_{g, 1} \\
y_{g, 2} \\
y_{g, 3} \\
\vdots \\
y_{g, j} \\
\vdots \\
y_{g, N}
\end{array}\right]=\left[\begin{array}{c}
h_{g, 1}\left(\underline{x}_{g}, \underline{\underline{p}}_{1}\right) \\
h_{g, 2}\left(\underline{x}_{g}, \underline{\hat{p}}_{2}\right) \\
h_{g, 3}\left(\underline{x}_{g}, \underline{\hat{p}}_{3}\right) \\
\vdots \\
h_{g, j}\left(\underline{x}_{g}, \underline{\hat{p}}_{j}\right) \\
\vdots \\
h_{g, N}\left(\underline{x}_{g}, \underline{\hat{p}}_{N}\right)
\end{array}\right]+\left[\begin{array}{c}
v_{g, 1} \\
v_{g, 2} \\
v_{g, 3} \\
\vdots \\
v_{g, j} \\
\vdots \\
v_{g, N}
\end{array}\right]
$$

or

$$
\underline{y}_{g}=\underline{h}_{g}\left(\underline{x}_{g}, \underline{\hat{p}}\right)+\underline{v}_{g}
$$


The random noise vector, $\underline{v}_{\mathrm{g}}$, is characterized by its mean and covariance:

$$
E\left[\underline{v}_{g}\right]=\underline{0} ; \quad E\left[\underline{v}_{g} \underline{v}_{g}^{T}\right]=R_{g}
$$

where the noise covariance matrix, $R_{\mathrm{g}}$, is typically a diagonal matrix. This characterization of $\underline{v}_{\mathrm{g}}$ is due to the fact that the effect of signals propagating at less than the speed of light is accounted for and incorporated into the model, and therefore it is reasonable to model the remaining ranging errors as zero-mean Gaussian errors. The magnitude of the entries of $R_{\mathrm{g}}$ is discussed later in the context of performance analysis

\subsubsection{The range-equivalent beat carrier phase model}

Beat carrier phase measurements are based on a comparison between measured changes in the received signal's phase and changes in the phase of a receiver-generated nominal replica signal. In effect, the beat carrier phase is the negative of the time integral of the received carrier Doppler shift [30]. This measurement involves an unknown bias term that originates from its integral nature.

The left panel of Fig. 5 illustrates the nature of the nominal signal frequency $f_{s}(t)$ as it is transmitted at a ground station (blue). It is characterized by a stair-stepping pattern that has short, smooth transition phases. The same pattern is generated at the receiver for its nominal replica signal. The frequency curve for the received signal (dashed green) is shifted rightward due to finite propagation time delay. Received frequencies are additionally slightly different due to Doppler shift that typically arises from the timevarying spatial distribution of electron density in the ionosphere. Integration of the signal's frequency over time yields a quantity that is regarded as the signal's phase, $\Phi(t)$, 
which is continuously computed for both the self-generated nominal replica signal and the received signal. The results, illustrated in the right panel of Fig. 5, take the form of similar, yet not identical, monotonically increasing curves.
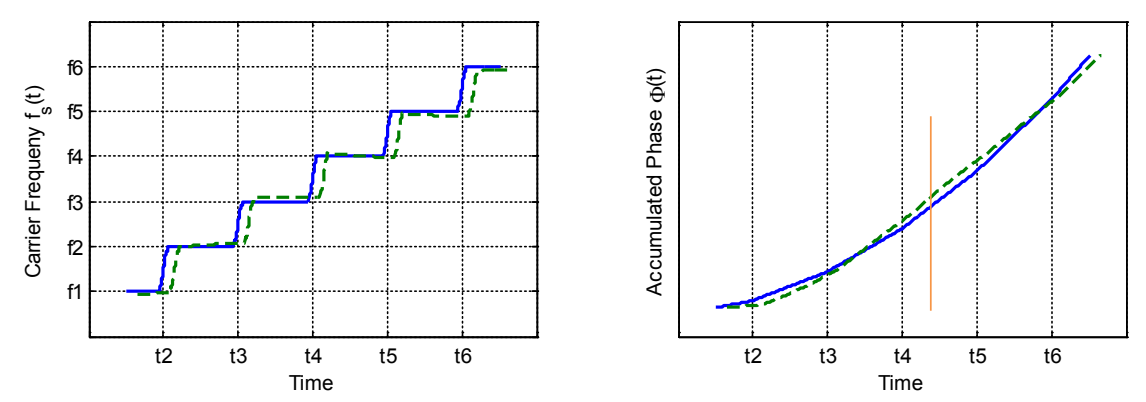

Figure 5: An example of the time-dependent signal frequency and the corresponding phase. The nominal transmitted frequency is shown in blue in the left-hand panel. The received frequency is shown as the dashed green curve in the same plot. The corresponding phases are shown in the right-hand panel.

The beat carrier phase is the difference between the two graphs at a given time. In the example above, this difference has been measured at some instance in time between times $t_{4}$ and $t_{5}$ at which frequency shifts occur for the nominal transmitted signal. The rangeequivalent beat carrier phase equals the product of this beat carrier phase measurement and the signal's wavelength for the nominal transmission frequency that applies between times $t_{4}$ and $t_{5}$.

Let $\rho_{c, j}=P_{\mathrm{f}, \mathrm{m}(j)}$ be the total true range-equivalent beat carrier phase of the $j^{\text {th }}$ ray-path, and let $y_{c, j}$ be the measured range-equivalent beat carrier phase of that ray-path. Recall that $P$ is computed by integrating the differential equation in Eq. (10). Let $\lambda_{\mathrm{w}, \mathrm{j}}$ be the corresponding signal's wavelength and let $\beta_{\mathrm{i}(\mathrm{j})}$ be an unknown bias term in units of wave cycles. Then the $j^{\text {th }}$ beat carrier phase measurement equation can be written as 


$$
\begin{aligned}
y_{c, j} & =h_{c, j}\left(\underline{x}_{c}, \underline{\hat{p}}_{j}\right)+v_{c, j}=\breve{h}_{c, j}\left(\underline{r}_{R}, \underline{\hat{p}}_{j}\right)+c \delta+\lambda_{w, j} \beta_{i(j)}+v_{c, j} \\
& =\tilde{h}_{c, j}\left[\underline{r}_{R}, \underline{\eta}_{m}\left(\underline{r}_{R}, \underline{\hat{p}}_{j}\right), \underline{\hat{p}}_{j}\right]+c \delta+\lambda_{w, j} \beta_{i(j)}+v_{c, j}
\end{aligned}
$$

where

$$
\underline{x}_{c} \triangleq\left[\begin{array}{c}
\underline{r}_{R} \\
c \delta \\
\underline{\beta}
\end{array}\right]
$$

and where the computed functions $\breve{h_{\mathrm{c}, \mathrm{j}}}$ and $\tilde{h_{\mathrm{c}, \mathrm{j}}}$ both model the ionosphere-refractioninduced range-equivalent carrier phase change of the $j^{\text {th }}$ ray-path $-\rho_{c, j}$. The vector $\underline{\beta}$ consists of all unknown bias terms that apply for all $N$ measurements. It should be noted that only one element of $\underline{\beta}$ is associated with a single measurement equation, but in most cases a single element of $\underline{\beta}$ will be common for several ray-paths, as discussed later. This fact will cause $\underline{\beta}$ to have fewer elements than the total number of ray-paths. The integer function $i(j)$ in the index of $\beta$ in Eq. (68) maps measurement ray-path indices $j$ to indices of their corresponding terms in $\underline{\beta}$.

As with group delay measurements, carrier phase measurement equations are stacked as an $\mathrm{N}$-dimensional vector equation that takes the form

$$
\left[\begin{array}{c}
y_{c, 1} \\
y_{c, 2} \\
y_{c, 3} \\
\vdots \\
y_{c, j} \\
\vdots \\
y_{c, N}
\end{array}\right]=\left[\begin{array}{c}
h_{c, 1}\left(\underline{x}_{c}, \underline{\hat{p}}_{1}\right) \\
h_{c, 2}\left(\underline{x}_{c}, \underline{\hat{p}}_{2}\right) \\
h_{c, 3}\left(\underline{x}_{c}, \underline{\hat{p}}_{3}\right) \\
\vdots \\
h_{c, j}\left(\underline{x}_{c}, \underline{\hat{p}}_{j}\right) \\
\vdots \\
h_{c, N}\left(\underline{x}_{c}, \underline{\hat{p}}_{N}\right)
\end{array}\right]+\left[\begin{array}{c}
v_{c, 1} \\
v_{c, 2} \\
v_{c, 3} \\
\vdots \\
v_{c, j} \\
\vdots \\
v_{c, N}
\end{array}\right]
$$


or, equivalently

$$
\left[\begin{array}{c}
y_{c, 1} \\
y_{c, 2} \\
y_{c, 3} \\
\vdots \\
y_{c, j} \\
\vdots \\
y_{c, N}
\end{array}\right]=\left[\begin{array}{c}
\breve{h}_{c, 1}\left(\underline{r}_{R}, \underline{\underline{p}}_{1}\right) \\
\breve{h}_{c, 2}\left(\underline{r}_{R}, \underline{\underline{p}}_{2}\right) \\
\breve{h}_{c, 3}\left(\underline{r}_{R}, \underline{\hat{p}}_{3}\right) \\
\vdots \\
\breve{h}_{c, j}\left(\underline{r}_{R}, \underline{\hat{p}}_{j}\right) \\
\vdots \\
\breve{h}_{c, N}\left(\underline{r}_{R}, \underline{\underline{p}}_{N}\right)
\end{array}\right]+\left[\begin{array}{c}
c \delta \\
c \delta \\
c \delta \\
\vdots \\
c \delta \\
\vdots \\
c \delta
\end{array}\right]+\left[\begin{array}{c}
\lambda_{1} \beta_{i(1)} \\
\lambda_{2} \beta_{i(2)} \\
\lambda_{3} \beta_{i(3)} \\
\vdots \\
\lambda_{j} \beta_{i(j)} \\
\vdots \\
\lambda_{N} \beta_{i(N)}
\end{array}\right]+\left[\begin{array}{c}
v_{c, 1} \\
v_{c, 2} \\
v_{c, 3} \\
\vdots \\
v_{c, j} \\
\vdots \\
v_{c, N}
\end{array}\right]
$$

The last equation can be written in shorthand notation as

$$
\underline{y}_{c}=\underline{h}_{c}\left(\underline{r}_{R}, \underline{\hat{p}}\right)+\underline{1} c \delta+\Lambda \underline{\beta}+\underline{v}_{c}
$$

where $\Lambda$ is a $N \mathrm{x} N_{\beta}$ matrix and $N_{\beta}$ is the dimension of $\underline{\beta}$. The random noise vector, $\underline{v}_{\mathrm{c}}$, is characterized by its mean and covariance

$$
E\left[\underline{v}_{c}\right]=\underline{0} ; E\left[\underline{v}_{c} \underline{v}_{c}^{T}\right]=R_{c}
$$

Finally, both groups of vector measurement equations, the first for the rangeequivalent group delays and the second for the range-equivalent beat carrier phases, can be grouped into a single $2 \mathrm{~N}$-dimensional vector equation. The same can be performed with the vector functions $\underline{h}_{\mathrm{g}}$ and $\underline{h}_{\mathrm{c}}$ and with the noise vectors $\underline{v}_{\mathrm{g}}$ and $\underline{v}_{\mathrm{c}}$ :

$$
\begin{aligned}
& \underline{y}=\left[\begin{array}{l}
\underline{y}_{g} \\
\underline{y}_{c}
\end{array}\right] ; \quad \underline{h}\left(\underline{x}_{c}, \underline{\hat{p}}\right)=\left[\begin{array}{l}
\underline{h}_{g}\left(\underline{x}_{g}, \underline{\hat{p}}\right) \\
\underline{h}_{c}\left(\underline{x}_{c}, \underline{\hat{p}}\right)
\end{array}\right] \\
& \underline{v}_{z}=\left[\begin{array}{l}
\underline{v}_{g} \\
\underline{v}_{c}
\end{array}\right] ; \quad R=E\left[\underline{v}_{z} \underline{v}_{z}^{T}\right]=\left[\begin{array}{cc}
R_{g} & 0 \\
0 & R_{c}
\end{array}\right]
\end{aligned}
$$


Note that $\underline{x}_{\mathrm{g}} \subset \underline{x}_{\mathrm{c}}$ so that $\underline{h}$ is conveniently defined as a function of $\underline{x}_{\mathrm{c}}$. The resulting measurement model takes the form

$$
\underline{y}=\underline{h}\left(\underline{x}_{c}, \underline{\hat{p}}\right)+\underline{v}_{z}
$$

\subsubsection{Measurement model sensitivity matrices}

Nonlinear gradient-based estimation algorithms, such as batch least-squares, require partial derivatives of the measurement model with respect to the unknown estimated quantities. These sensitivities must be computed at a succession of improved guesses of the optimal estimates of the unknowns. In the present context, the required partial derivatives are those of each $h_{j}$ measurement model function with respect to the elements of the unknown $\underline{x}$ and $\underline{\underline{p}}$ vectors. The partial derivative with respect to the $c \delta$ element of $\underline{x}$ is 1 , consistent with Eq. (62). Other derivatives, those with respect to the elements of $\underline{r}_{\mathrm{R}}$ and $\underline{\hat{p}}$, require special calculations.

Throughout the following derivation $\underline{\eta}_{m}$ is the stacked vector of coordinate functions for all bounce points of the $j^{\text {th }}$ ray-path, and $\hat{\underline{p}}_{\mathrm{j}}$ is the vector of applicable ionosphere parameters, both have been defined earlier. Consistent with Fig. 4 , the two vectors $\underline{v}_{f, j}$ and $\underline{v}_{0, j}$ are stacked vectors that hold the components of the Cartesian representation of the unit direction vectors for the incoming and reflected rays of all bounce points of that raypath.

The sensitivity of the $j^{\text {th }}$ range measurement to the input variables $\underline{r}_{\mathrm{R}}$ and $\hat{\underline{p}}_{\mathrm{j}}$ is obtained by differentiating Eq. (62): 


$$
\begin{aligned}
& \frac{D \underline{h}_{j}}{D \underline{r}_{R}}=\frac{\partial \underline{\tilde{h}}_{j}}{\partial \underline{\eta}_{m}} \frac{\partial \underline{\eta}_{m}}{\partial \underline{r}_{R}}+\frac{\partial \underline{\tilde{h}}_{j}}{\partial \underline{r}_{R}} \\
& \frac{D \underline{h}_{j}}{D \underline{\underline{p}}_{j}}=\frac{\partial \underline{\tilde{h}}_{j}}{\partial \underline{\eta}_{m}} \frac{\partial \underline{\eta}_{m}}{\partial \underline{\underline{p}}_{j}}+\frac{\partial \underline{\tilde{h}}_{j}}{\partial \underline{\underline{p}}_{j}}
\end{aligned}
$$

Note how Eq. (76) differs from the equivalent Eq. (14) in Ref. [5] because it includes a direct dependence of $\tilde{\underline{h}}_{\mathrm{j}}$ on $\underline{\underline{j}}_{\mathrm{j}}$ that does not exist with the simplified ray-path model of the earlier work.

Some of the terms on the right hand side of this set of two equations require a complicated evaluation procedure. Recalling the variable dependencies in the model, the $g_{j}$ system of equations for the $j^{\text {th }}$ ray-path can be written as

$$
\begin{gathered}
\underline{0}=\underline{g}_{j}=\underline{g}_{j}\left(\underline{v}_{f, j}\left[\underline{\eta}_{m}\left(\underline{\hat{p}}_{j}, \underline{r}_{R}\right), \underline{\hat{p}}_{j}\right], \underline{v}_{0, j}\left[\underline{\eta}_{m}\left(\underline{\hat{p}}_{j}, \underline{r}_{R}\right), \underline{\hat{p}}_{j}, \underline{r}_{R}\right]\right. \\
\left., \underline{\eta}_{m}\left(\underline{\hat{p}}_{j}, \underline{r}_{R}\right), \underline{u}_{j}\left[\underline{\eta}_{m}\left(\underline{\hat{p}}_{j}, \underline{r}_{R}\right)\right]\right)
\end{gathered}
$$

Differentiating Eq. (77) with respect to $\underline{\underline{r}}_{\mathrm{R}}$ and $\underline{\underline{p}}_{\mathrm{j}}$ using the chain rule yields linear equations for the unknown partial derivatives $\partial \underline{\eta}_{\mathrm{m}} / \partial \underline{\underline{R}}_{\mathrm{R}}$ and $\partial \underline{\eta}_{\mathrm{m}} / \partial \hat{\underline{p}}_{j}$. Solution of these equations yields the formulas: 


$$
\begin{aligned}
& \frac{\partial \underline{\eta}_{m}}{\partial \underline{r}_{R}}=-\left(\frac{\partial \underline{g}_{j}}{\partial \underline{v}_{f, j}} \frac{\partial \underline{v}_{f, j}}{\partial \underline{\eta}_{m}}+\frac{\partial \underline{g}_{j}}{\partial \underline{v}_{0, j}} \frac{\partial \underline{v}_{0, j}}{\partial \underline{\eta}_{m}}+\frac{\partial \underline{g}_{j}}{\partial \underline{u}_{j}} \frac{\partial \underline{u}_{j}}{\partial \underline{\eta}_{m}}+\frac{\partial \underline{g}_{j}}{\partial \underline{\eta}_{m}}\right)^{-1} \\
& \cdot\left(\frac{\partial \underline{g}_{j}}{\partial \underline{v}_{0, j}} \frac{\partial \underline{v}_{0, j}}{\partial \underline{r}_{R}}\right) \\
& \frac{\partial \underline{\eta}_{m}}{\partial \underline{\hat{p}}_{j}}=-\left(\frac{\partial \underline{g}_{j}}{\partial \underline{v}_{f, j}} \frac{\partial \underline{v}_{f, j}}{\partial \underline{\eta}_{m}}+\frac{\partial \underline{g}_{j}}{\partial \underline{v}_{0, j}} \frac{\partial \underline{v}_{0, j}}{\partial \underline{\eta}_{m}}+\frac{\partial \underline{g}_{j}}{\partial \underline{u}_{j}} \frac{\partial \underline{u}_{j}}{\partial \underline{\eta}_{m}}+\frac{\partial \underline{g}_{j}}{\partial \underline{\eta}_{m}}\right)^{-1} \\
& \cdot\left(\frac{\partial \underline{g}_{j}}{\partial \underline{v}_{f, j}} \frac{\partial \underline{v}_{f, j}}{\partial \underline{p}_{j}}+\frac{\partial \underline{g}_{j}}{\partial \underline{v}_{0, j}} \frac{\partial \underline{v}_{0, j}}{\partial \underline{p}_{j}}\right)
\end{aligned}
$$

These expressions are substituted into the right-hand side of Eq. (76) to complete the formulas for the required sensitivity matrices $\partial \underline{h}_{\mathrm{j}} / \partial \underline{r}_{\mathrm{R}}$ and $\partial \underline{h}_{\mathrm{j}} / \partial \underline{\underline{p}}_{\mathrm{j}}$

Of the terms on the right hand side of Eq. (78), the following terms can be evaluated analytically through differentiation of the bounce point equations: $\partial g_{\mathrm{j}} / \partial \underline{v}_{\mathrm{f}, \mathrm{j}}, \partial \mathrm{g}_{\mathrm{j}} / \partial \underline{v}_{0, \mathrm{j}}$ and $\partial g_{\mathrm{j}} / \partial \underline{u}_{\mathrm{j}}$. Ease in computing these sensitivity Jacobians is essentially the motivation for using the reflection formulation of Eqs. (30)-(35).

The following terms, however, can only be evaluated in tandem with the raytracing calculations that determine single ray hops: $\partial \underline{v}_{\mathrm{f}, \mathrm{j}} / \partial \underline{\underline{p}}_{\mathrm{j}}, \partial \underline{v}_{0, \mathrm{j}} / \partial \underline{\underline{j}}_{\mathrm{j}}, \partial \underline{v}_{\mathrm{f}, \mathrm{j}} / \partial \underline{\eta}_{\mathrm{m}}, \partial \underline{v}_{0, \mathrm{j}} / \partial \underline{\eta}_{\mathrm{m}}$ and $\partial \underline{v}_{0, j} / \partial \underline{r}_{\mathrm{R}}$. The computation of these terms has been described in Section 4.2.6 that presented single ray-hop numerical computations. That section also discussed the computation of the terms $\partial \underline{h}_{\mathrm{j}} / \partial \underline{\eta}_{\mathrm{m}}, \partial \underline{h}_{\mathrm{j}} / \partial \underline{r}_{\mathrm{R}}$ and $\partial \underline{h}_{\mathrm{j}} / \partial \underline{\hat{p}}_{\mathrm{j}}$ that appear on the right hand side of Eq. (76). Computation of $\partial g_{\mathrm{j}} / \partial \eta_{\mathrm{m}}$ has been shown in Subsection 3.4.1 in the context of solving for the implicit functions $\underline{\eta}$. Finally, the term $\partial \underline{u}_{\mathrm{j}} / \partial \underline{\eta}_{\mathrm{m}}$ is derived from the Earth's surface model. 


\section{CHAPTER 4}

\section{BATCH ESTIMATION OF RECEIVER POSITION, RECEIVER CLOCK OFFSET, AND IONOSPHERE PARAMETERS}

\subsection{Batch Filter Problem and Solution}

\subsubsection{The nominal case}

A batch filter has been developed. It estimates $\underline{x}_{\mathrm{c}}$ (or $\underline{x}_{\mathrm{g}}$ when only considering groupdelay measurements) and $\underline{p}$ by minimizing a cost function that includes weighted squared differences between the measurements and their modeled values, and between the estimated $\underline{\underline{p}}$ elements and their a priori estimates. In the general case, the batch filtering problem seeks the values that jointly minimize the cost function

$$
J_{1}\left(\underline{x}_{c}, \underline{p}\right)=\frac{1}{2}\left[\underline{y}-\underline{h}\left(\underline{x}_{c}, \underline{p}\right)\right]^{\mathrm{T}} R^{-1}\left[\underline{y}-\underline{h}\left(\underline{x}_{c}, \underline{p}\right)\right]+\frac{1}{2} \zeta(\underline{p}-\underline{\bar{p}})^{\mathrm{T}} M^{-1}(\underline{p}-\underline{\bar{p}})
$$

where $y$ is the $2 N \times 1$ stacked vector of the $N$ measured pseudoranges and $N$ measured range-equivalent beat carrier phases for the given $N$ ray-paths. $R$ is the square, symmetric, $2 N$-by- $2 N$, positive definite measurement error covariance matrix (typically a diagonal matrix), $\overline{\underline{p}}$ is the a priori estimate of the ionosphere parameter vector, and $M$ is the square, symmetric, positive definite covariance matrix that models the uncertainty in the a priori ionosphere parameter vector $\overline{\underline{p}}$. The elements of $\underline{p}$ consist of ionosphere parameters which apply in the vicinity of the unknown, true signal ray-paths. The $M$ matrix has row 
and column dimensions equal to the dimension of $\overline{\underline{p}}$. The role of the positive scalar valued weighting coefficient $\zeta$ will be discussed later.

The batch least-squares cost function of Eq. (79) does not include a priori values of the elements of $\underline{x}_{\mathrm{c}}$ with penalties for differences between those values and the estimated $\underline{x}_{\mathrm{c}}$. This means that no prior knowledge about these terms is assumed, just as in standard GPS point navigation solutions.

The minimizing solution to the estimation problem in Eq. (79) is equivalent to the optimal least-squares solution to the following over-determined system of nonlinear equations:

$$
\left[\begin{array}{c}
R^{-1 / 2} \underline{y} \\
\sqrt{\zeta} M^{-1 / 2} \underline{\bar{p}}
\end{array}\right]=\left[\begin{array}{c}
R^{-1 / 2} \underline{h}\left(\underline{x}_{c}, \underline{p}\right) \\
\sqrt{\zeta} M^{-1 / 2} \underline{p}
\end{array}\right]+\underline{v}_{1}
$$

where $R^{-1 / 2}$ and $M^{-1 / 2}$ are the inverses of the Cholesky factor square roots of, respectively, the matrices $R$ and $M$, and where $\underline{v}_{1}$ is a zero-mean, identity-covariance Gaussian random error vector whose norm squared is minimized by the batch solution.

The problem is solved with a modified version of the gradient-based Gauss-Newton method that is described in the next section.

\subsubsection{The case of a known receiver position and clock error}

In some cases it is desirable to solve for the unknown ionosphere model (and, potentially, for the unknown carrier bias terms) while the receiver location and clock 
offset are assumed known and fixed (or, at least, closely monitored and corrected). In such cases, the optimization problem takes the general form

$$
J_{4}\left(\underline{x}_{f}, \underline{p}\right)=\frac{1}{2}[\underline{y}-\underline{h}(\underline{\beta}, \underline{p})]^{\mathrm{T}} R^{-1}[\underline{y}-\underline{h}(\underline{\beta}, \underline{p})]+\frac{1}{2} \zeta(\underline{p}-\underline{\bar{p}})^{\mathrm{T}} M^{-1}(\underline{p}-\underline{\bar{p}})
$$

If carrier phase measurements are not processed, the problem will reduce to estimation of ionosphere parameters only.

\subsection{A Modified Gauss-Newton Method}

The Gauss-Newton method has been used to solve this estimation problem. This method is described in Refs. [31] and [32]. It is additionally discussed in the context of convex function optimization through nonlinear programing in Ref. [33]. It is a gradientbased iterative method. Some adaptations to this method have been made in order to address the special characteristics of the minimization problem, as described in the following subsections.

\subsubsection{The top-level procedure}

Each iteration starts with guesses of the optimizing values of $\underline{x}_{\mathrm{c}}$ and $\underline{p}$. First, it linearizes Eq. (80) about these guessed values. Next, it solves the resulting overdetermined linear least-squares problem to get candidates for improved solution guesses of $\underline{x}_{\mathrm{c}}$ and $\underline{p}$. Finally, it searches along the line in $\left[\underline{x}_{\mathrm{c}} ; \underline{p}\right]$ space from the old guess to the candidate new guess in order to find a new guess that reduces the cost $J_{1}\left(\underline{x_{c}}, \underline{p}\right)$. 
Linearization of Eq. (80) about the current guess for the unknowns $\underline{x}_{\mathrm{c}}$ and $\underline{p}, \underline{x}_{\mathrm{c}, \text { guess }}$ and $\underline{p}_{\text {guess, }}$ takes the form

$$
\begin{aligned}
& {\left.\left[\begin{array}{cc}
R^{-1 / 2} & 0 \\
0 & \sqrt{\zeta} M^{-1 / 2}
\end{array}\right]\left[\begin{array}{cc}
\frac{\partial \underline{h}\left(\underline{x}_{c}, \underline{p}\right)}{\partial \underline{x}_{c}} & \frac{\partial \underline{h}\left(\underline{x}_{c}, \underline{p}\right)}{\partial \underline{p}} \\
0 & I
\end{array}\right]\right|_{\underline{x}_{c, \text { guess }} \underline{\underline{p}}_{\text {guess }}}\left[\begin{array}{c}
\Delta \underline{x}_{c} \\
\Delta \underline{p}
\end{array}\right]=} \\
& {\left[\begin{array}{cc}
R^{-1 / 2} & 0 \\
0 & \sqrt{\zeta} M^{-1 / 2}
\end{array}\right]\left[\begin{array}{c}
\underline{y}-\underline{h}\left(\underline{x}_{c, \text { guess }}, \underline{p}_{\text {guess }}\right) \\
\underline{p}-\underline{p}
\end{array}\right] }
\end{aligned}
$$

This over-determined system of equations is solved through a short series of operations. Starting with a QR factorization, as in Ref. [31]:

$$
Q_{b}\left[\begin{array}{c}
R_{b} \\
0
\end{array}\right]=\left.\left[\begin{array}{cc}
R^{-1 / 2} & 0 \\
0 & \sqrt{\zeta} M^{-1 / 2}
\end{array}\right]\left[\begin{array}{cc}
\frac{\partial \underline{h}\left(\underline{x}_{c}, \underline{p}\right)}{\partial \underline{x}} & \frac{\partial \underline{h}\left(\underline{x}_{c}, \underline{p}\right)}{\partial \underline{p}} \\
0 & I
\end{array}\right]\right|_{\underline{x}_{c, \text { guess }}, \underline{p}_{\text {guess }}}
$$

Let

$$
\underline{z}_{a}=\left[\begin{array}{cc}
R^{-1 / 2} & 0 \\
0 & \sqrt{\zeta} M^{-1 / 2}
\end{array}\right]\left[\begin{array}{c}
\underline{y}-\underline{h}\left(\underline{x}_{c, \text { guess }}, \underline{p}_{\text {guess }}\right) \\
\bar{p}-\underline{p}
\end{array}\right]
$$

then the vectors $\underline{z}_{\mathrm{b}}$ and $\underline{z}_{\mathrm{r}}$ are computed as follows:

$$
\left[\begin{array}{c}
\underline{z}_{b} \\
\underline{z}_{r}
\end{array}\right]=Q_{b}^{T} \underline{\underline{z}}_{a}
$$

such that the dimension of $\underline{z}_{\mathrm{b}}$ is the same as the row/column dimension of $R_{\mathrm{b}}$. The initial Gauss-Newton step is obtained by computing 


$$
\left[\begin{array}{c}
\Delta \underline{x}_{c} \\
\underline{\underline{p}}
\end{array}\right]=R_{b}^{-1} \underline{z}_{b}
$$

New candidates for the optimal solution are computed using

$$
\left[\begin{array}{c}
\underline{x}_{c, \text { guess }} \\
\underline{p}_{\text {guess }}
\end{array}\right]_{\text {new }}=\left[\begin{array}{c}
\underline{x}_{c, \text { guess }} \\
\underline{p}_{\text {guess }}
\end{array}\right]+\alpha\left[\begin{array}{c}
\Delta \underline{x}_{c} \\
\Delta \underline{p}
\end{array}\right]
$$

where $\alpha=0.5^{n_{\mathrm{GN}}}, n_{\mathrm{GN}}=0,1,2, \ldots$ is a scaling factor that is used to guarantee actual reduction in the cost function $J_{1}\left(\underline{x_{c}}, \underline{p}\right)$.

The processes of determining $\alpha$ and a resulting new guess for the unknown parameters is performed in an auxiliary loop as follows. First, the corrected state vector is evaluated using Eq. (87), substituting 0 for $n_{\mathrm{GN}}$ in the expression for $\alpha$. The resulting vector is then used to reevaluate the cost function and compare it with the cost function's current value, i.e., the value that has been computed at the end of the previous Gauss-Newton step. If cost decrease is achieved, then the processes is terminated and the new state vector that has been computed for $n_{\mathrm{GN}}=0$ becomes the new guess for the unknown parameters. Otherwise, $\alpha$, the resulting new guess for the unknowns, and $J_{1}$ are evaluated for $n_{\mathrm{GN}}=1$. The cost function is again compared with its current value. This process will terminate once cost reduction is achieved. This line search between the old guess and the new candidate guess ensures convergence to a local minimum of $J_{1}\left(\underline{x}_{c}, \underline{p}\right)$ as the theory of the Gauss-Newton method guaranties the existence of an $n_{\mathrm{GN}}$ for which $J_{1}$ is reduced - see Refs. [32] and [34]. 
The top-level procedure is repeated iteratively until the cost function is minimized. The Gauss-Newton method with step-size halving is guaranteed to converge to a local minimum, but the minimum is not guaranteed to be global. Testing experience indicates that convergence to local minimum that is different from the global minimum occurs rarely, if ever. Therefore, the algorithm seems to be insensitive to the initial guess and the corresponding magnitude of the initial error and is nearly guaranteed to converge to its global minimum. Validity of the latter statement is further demonstrated in the discussion of Section 6.4 .

\subsubsection{Measurements exclusion}

This is the first place where the algorithm deviates from the classic Gauss-Newton method. The modified method uses an ad hoc approach that allows the sets of considered measurements to change during the iterative process. This requires modifications to the way cost function reduction is approached.

In the context of this study, the Gauss-Newton process is performed only once the considered transmitters/receiver/ionosphere-model setup has proven to be physically feasible, meaning that valid ray-paths have been successfully computed for all transmitted signals. This means that failures in algorithm attempts to solve for the location of the bounce points $\hat{\underline{\eta}}_{\mathrm{j}}$ of the $j^{\text {th }}$ ray-path during the iterative process cannot be attributed to physical non-feasibility. As earlier shown, solution for $\hat{\eta}_{\mathrm{j}}$ is obtained with the ray-path solver. Occasionally, the ray-path solver may fail to solve for $\hat{\eta}_{j}$ when called by the primary, top-level Gauss-Newton process. This it is typically due to either a poor estimate for the location of the receiver and the ionospheric parameters, or difficulties in 
the numerical raytracing computation for one or more of that ray-path's ray-hops. In less common cases the ray-path solver might fail due a poorly chosen initial guess for the inner Gauss-Newton process of the solver.

Regardless of the cause, in such cases the particular measurements that failed to be computable in the filter's model are temporarily excluded from the set of measurements that are considered. Such exclusions apply only at the current optimal step-size determination step of the current Gauss-Newton iteration, where a particular value of $\alpha$ is considered to evaluate a guess for the unknown parameters and the resulting cost function. This means that an excluded measurement may be re-included in the next stepsize determination step where a different value for $\alpha$ is used, and will necessarily be included in the next top-level Gauss-Newton iteration, where measurement models and their sensitivity matrices are reevaluated.

Measurement exclusion means that cost function computations may take different forms at different iterations, as different subsets of measurements are considered. The consequent process of comparing evaluated cost functions at different iterations and different step-size determination steps is as follows.

Suppose that the $(b-1)^{\text {st }}$ Gauss-Newton iteration has been completed. The $b^{\text {th }}$ GaussNewton iteration then begins with calculations that include an attempt to solve for all raypaths' bounce-points $\hat{\eta}_{\mathrm{j}}, j=1, \ldots, N$ for all $N$ available ray-path measurements. This attempt is processed with estimates for $\underline{x}$ and $\underline{p}$ that have been obtained at the end of the $(b-1)^{\text {st }}$ iteration, designated $\underline{x}_{\mathrm{b}-1, \text { final }}$ and $\underline{p}_{\mathrm{b}-1, \mathrm{final}}$. Let $\underline{\mathrm{b}}_{\mathrm{b}, 0}$ be the subset of the full set of the

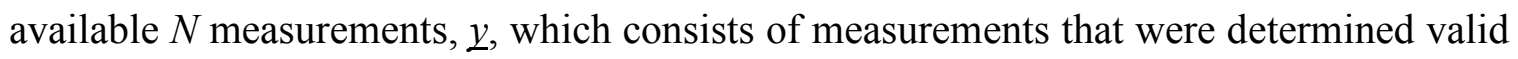


in this attempt by the success of the algorithm at solving for their bounce points. $\underline{y b}, 0$ has $N_{\mathrm{b}, 0} \leq N$ elements, and the associated cost function that is computed for this subset with $\underline{x}_{\mathrm{b}-}$ 1 ,final and $\underline{p}_{\mathrm{b}-1, \text { final }}$ is denoted $J(\mathrm{~b}, 0)$. A series of computations for the current $b^{\text {th }}$ iteration is performed next, resulting in a preliminary set of two proposed correction vectors that could potentially become the new estimates: $\underline{x}_{\mathrm{b}, 0}$ and $\underline{p_{\mathrm{b}}, 0}$. Next, an inner loop is executed to determine this iteration's optimal step size, i.e., a positive scaling factor $\alpha \leq 1$ for these correction vectors. This is the same sub-process that was briefly described earlier in Subsection 4.2.1, omitting the details of how the cost function is evaluated. In the first iteration of that inner loop, the step size factor $\alpha$ is taken as 1 , and computations for all ray-paths corresponding with $y_{b, 0}$ are then carried out. Those measurements that were determined valid constitute a new subset, $\underline{\mathrm{b}}_{\mathrm{b}, 1}$, that has $N_{\mathrm{b}, 1}$ elements where $N_{\mathrm{b}, 1 \leq N_{\mathrm{b}, 0}}$ and $y_{\mathrm{b}, 1} \subseteq \underline{y}_{\mathrm{b}, 0 .} . J(\mathrm{~b}, 1)$ is the computed value for the cost function that is now evaluated for $\underline{\mathrm{b}}_{\mathrm{b}, 1}$ with $\underline{x}_{b, 1}$ and $\underline{p}_{\mathrm{b}, 1}$ - the new estimates for $\underline{x}$ and $\underline{p}$ that have been computed with $\alpha=1$. Still in the first iteration of the inner loop, it is required to determine whether a reduction in the cost function has been achieved, by comparing $J(b, 1)$ with the cost function that has been evaluated for the previous Gauss-Newton iteration. However, comparison can only be performed when the two cost functions are evaluated with the same sets of measurements, and therefore the cost function must be reevaluated with $\underline{x}_{\mathrm{b}-1, \text { final }}$ and $\underline{p}_{\mathrm{b}}$ 1 ,final as state estimates and $y_{\mathrm{b}, 1}$ as the set of applicable measurements. The result, denoted $J_{\text {reeval }}(\mathrm{b}-1,1)$, is now compared with $J(\mathrm{~b}, 1)$. If $J(\mathrm{~b}, 1)$ is less than $J_{\text {reeval }}(\mathrm{b}-1,1)$, then the inner loop is terminated and $\underline{x}_{b, 1}$ and $\underline{p}_{b, 1}$ become the updated estimates for the state vectors, designated $\underline{x}_{\mathrm{b}, \text { final }}$ and $\underline{b}_{\mathrm{b} \text {,final. }}$ Otherwise, $\alpha$ is halved, and the process for the inner loop is repeated with $\alpha=0.5$. This second iteration of the inner loop includes: (a) 
determination of valid ray-paths and the resulting $N_{\mathrm{b}, 2}$ and $\underline{y}_{\mathrm{b}, 2}$ where $\underline{y}_{\mathrm{b}, 2} \subseteq \underline{y}_{\mathrm{b}, 0}$, (b) evaluation of the cost function $J(b, 2)$ with $\underline{x}_{\mathrm{b}, 2}$ and $\underline{p}_{\mathrm{b}, 2}$ - corrected state vectors that were computed with $\alpha=0.5$, (c) a second reevaluation of the cost function $J_{\text {reeval }}(\mathrm{b}-1,2)$ with $\underline{x}_{\mathrm{b}}$ 1,final, $\underline{b}_{\mathrm{b}-1, \text { final }}$ and $\underline{y}_{\mathrm{b}, 2}$, and finally (d) a comparison between $J(\mathrm{~b}, 2)$ and $J_{\text {reeval }}(\mathrm{b}-1,2)$. If $J(b, 2)$ is less than $J_{\text {reeval }}(b-1,2)$, the inner loop is terminated and $\underline{x}_{b, 2}$ and $\underline{p}_{\mathrm{b}, 2}$ become the updated estimates for the state vectors. Otherwise, the process will repeat itself until $J(\mathrm{~b}, \mathrm{k})$ is less than $J_{\text {reeval }}(\mathrm{b}-1, \mathrm{k})$ for some $k$. Once reduction in the cost function has been achieved, the algorithm will proceed to the next Gauss-Newton iteration.

By design this method is guaranteed to ensure a decrease in the cost function for two sequential Gauss-Newton steps for a subset of measurements, but it is theoretically possible that later steps would result in a higher computed cost function value because different subsets of measurements may be used to enforce a cost decrease during different steps. Theoretically, this optimization strategy removes the guarantees of convergence for a Gauss-Newton method with step size halving, but it has not been observed to result in significant convergence issues. The scope of validity for this assumption is examined in Sections 6.2 and 6.3 that discuss algorithm functionality.

An alternative, more stringent approach to dealing with unsolvable ray-paths would be excluding measurements, as with the current approach, except here measurements that have been excluded in previous Gauss-Newton iterations would not be considered in later iterations, until solution convergence has been reached. An obvious drawback for this approach is the possibility that exclusion of too many measurements may negatively 
affect the iterative optimization process by degrading system observability and potentially prevent convergence.

\subsubsection{Measurement rejection}

Measurement rejection mechanisms are common practice in sensor-based systems. The potential of significant, un-modeled measurement errors that affect sensor readings is typically handled with likelihood tests that are designed to detect and reject outliers as bad data. Likelihood tests rely on measurement error models, where abnormal measurements take values that are very improbable based on those models and, therefore, are rejected.

While un-modeled sensor errors are not considered within the scope of this study, it has been observed that due to known algorithm limitations, significant discrepancies between computed and measured group delays / phase advances may arise, resulting in sub-optimal corrections to the unknowns that occasionally cause solution divergence (see Subsection 6.3.2). Unlike with the case of anomalous sensor reading errors, these discrepancies originate from erroneous ranging predictions that are computed by the algorithm.

Regardless of the cause, it is crucial that the algorithm will be able to detect any such occurrences and handle them in a manner that is similar to the procedure that has been discussed in Subsection 4.2.2. The most significant difficulty in detecting erroneous computed pseudoranges is the fact that, due to their nature, these errors cannot be reliably modeled or predicted. Let the $j^{\text {th }}$ measurement's residual be defined as 


$$
v_{j}=y_{j}-h_{j}\left(\underline{x}_{c}, \underline{\underline{p}}_{j}\right)
$$

While the magnitude of valid $v_{\mathrm{j}}$ terms will generally decrease in the iterative process, it has been observed that the magnitude of invalid $v_{\mathrm{j}}$ terms, for which $h_{\mathrm{j}}$ is miscalculated, varies in a manner that is not aligned with apparent trends in the way valid terms change. Therefore, a procedure designed to detect erroneous computed ranges cannot rely on their absolute magnitudes. Instead, the algorithm uses a relative criterion where the normalized magnitudes of all residual terms are compared.

Consistent with the notation of Subsection 4.2.2, let $y \mathrm{~b}, \mathrm{~d}$ be the set of measurements applying at the $d^{\text {th }}$ inner loop of the $b^{\text {th }}$ Gauss-Newton iteration. $y_{\mathrm{b}, \mathrm{d}}$ has $N_{\mathrm{b}, \mathrm{d}}$ elements, corresponding to measurements that have been determined to be valid in a procedure of the kind that has been described in the previous subsection. Next, residual terms for $y_{b, d}$ are computed and stored as a vector $-\underline{v_{b}, \mathrm{~d}}$. The element exclusion operator $\backslash$ is used to define a new set of vectors so that the vector $\underline{v}_{b, d_{j}}=\left(\underline{v}_{b, d} \mid v_{b, d_{j}}\right)$ includes all elements of $\underline{v}_{b, d}$ except for its $j^{\text {th }}$ element $-v_{\mathrm{b}, \mathrm{d}_{\mathrm{j}}}$. The mean of the vector $\underline{v}_{\mathrm{b}, \mathrm{d}_{\mathrm{j}}}$ is a scalar that is designated $\overline{v_{b}, d_{\mathrm{j}}}$. The $j^{\text {th }}$ term of an auxiliary set of positive elements is computed by

$$
\kappa_{b, d_{j}}=\frac{\left|v_{b, d_{j}}\right|}{\left\{\frac{1}{N_{b, d}-1} \sum_{i=1}^{N_{b, d}-1}\left(\underline{v}_{b, d_{j}(i)}-\bar{v}_{b, d_{j}}\right)^{2}\right\}^{1 / 2}}
$$


where $\underline{v}_{b, d_{j}(i)}$ is $\underline{v}_{b, d_{j}}$ 's $i^{\text {th }}$ element. On a more intuitive note, $\kappa_{b, d_{j}}$ measures the magnitude of the $j^{\text {th }}$ measurement's residual, normalized by the standard deviation that is computed for all other residual terms.

Measurements whose corresponding $\kappa$ values are larger than 6 are excluded from $y_{\mathrm{b}, \mathrm{d}}$ for the remaining procedure of the $d^{\text {th }}$ inner loop step. This method has proven useful with many, yet not all, cases where excessive residual terms prevented the solution from converging.

\subsubsection{Calculation of the $M$ Matrix}

As briefly mentioned earlier in Subsection 2.3.2, the $M$ matrix is constructed from the $M_{0}$ matrix that was introduced in that subsection by considering only the rows and columns of $M_{0}$ that correspond with active grid nodes' parameters. Consequently, $M$ may take different forms in different top-level Gauss-Newton iterations since ray-path trajectories are re-computed at the beginning of each iteration. This should be accounted for when cost function values are compared as discussed in Subsection 4.2.2.

An alternative approach would be to pre-determine the active grid nodes that may be used throughout the entire process. This would result in static definitions of the $\underline{p}$ vectors and $M$ matrices.

\subsubsection{The varying cost function}

The motivation for including the right-most term in Eq. (79) is to ensure that corrections that are applied to the ionospheric Chapman parameters (and their latitude and longitude partial derivatives) are reasonable. Recognizing the potentially limited 
observability for some of the parameters, one would like to limit the magnitude of corrections by including this terms that panelizes for big differences between the a priori and the current estimates for these parameters.

In the previous, simplified version of the model that was used in Ref. [5], the $\zeta$ term in Eq. (79) was equal to 1. Experiments carried out for the current model indicated, however, that the significant nonlinearity which characterizes the model, along with the fact that the ionosphere model terms in the state vector are natural logarithms of physical quantities, could result in unreasonable corrections being applied to those parameters, ultimately causing frequent algorithm divergence. It has been observed that excessive corrections are mostly applied right after the algorithm step where the search switches from the limited-corrections mode of Case 2, as in Subsection 4.2.7, into the fullcorrections mode of Case 1, as in Section 4.1. These findings indicated a useful ad hoc modification for the $\zeta$ term. Many experiments were conducted in order to determine a reasonable formulation for $\zeta$. With the formula

$$
\zeta=1+10^{x-k i}
$$

where $\chi=5$ and $k i$ is the index of the Gauss-Newton iterations that is initialized to 1 at the first full-corrections mode iteration, a high convergence rate for the whole algorithm has been achieved. It should be noted that the use of this term should not have any impact on the optimal solution, in comparison to the case of $\zeta=1$, as it converges quickly to 1 as $k i$ increases. For the initial steps with small $k i$, this modification to $\zeta$ has the effect of limiting the magnitudes of the ionosphere parameter corrections during the initial phases of the algorithm. 


\subsubsection{Varying sets of carrier bias terms}

It is possible that due to either measurement exclusion, as in Section 4.2.2, or measurements rejection, as in Section 4.2.3, for a given Gauss Newton iteration none of the measurements that are related to a particular bias term $\beta_{\mathrm{e}}$ are used. This would make $\beta_{\mathrm{e}}$ temporarily unobservable and therefore the algorithm will exclude that term from that iteration's states vector.

\subsubsection{Iterating when position solution is far from convergence}

Recognizing the limitations of the first-order Gauss-Newton method when it comes to arriving at a solution starting from a guess that is far from the receiver's true location, the algorithm distinguishes between two cases. In the nominal Case 1 that has been described above, the position solution is assumed to be close to convergence. In this case, the algorithm will consider variations in the three components of the ECEF representation of the receiver's location $\underline{r}_{\mathrm{R}}$, variations in the range-equivalent receiver clock offset $c \delta$, variations in the carrier phase measurement biases $\underline{\beta}$, and variations in the ionosphere parameters of all bi-quintic spline nodes that affect the ray-paths. In Case 2, the position solution is assumed to be far from convergence. Consequently, only group-delay measurements will be processed. In addition, the algorithm will only consider variations in the receiver position's latitude and longitude and in its clock bias. Variations of altitude and of ionospheric model parameters are excluded. Experience has demonstrated that this modification tends to ensure convergence when starting far from the solution. The simplified cost function for this simplified search takes the following form: 


$$
J_{2}\left(\underline{x}_{g}\right)=\frac{1}{2}\left[\underline{y}_{g}-\underline{h}_{g}\left(\underline{x}_{g}, \underline{\bar{p}}\right)\right]^{\mathrm{T}} R^{-1}\left[\underline{y}_{g}-\underline{h}_{g}\left(\underline{x}_{g}, \underline{\bar{p}}\right)\right]
$$

The corresponding over-determined system of equations in linearized latitude/longitude form is

$$
R_{g}^{-1 / 2}\left[\underline{y}_{g}-\underline{h}_{g}\left(\underline{x}_{g, \text { guess }}, \underline{p}\right)\right]=\left.R_{g}^{-1 / 2} \frac{\partial \underline{h}}{\partial \underline{x}_{g}}\right|_{\left(\underline{x}_{g, \text { guess }}, \underline{p}\right)}\left[\begin{array}{ccc}
\frac{\partial \underline{r}}{\partial \phi} & \frac{\partial \underline{r}}{\partial \lambda} & 0 \\
0 & 0 & 1
\end{array}\right]\left[\begin{array}{c}
\Delta \phi \\
\Delta \lambda \\
\Delta(c \delta)
\end{array}\right]+\underline{v}_{2}
$$

where $\underline{x}_{g, \text { guess }}=\left[\underline{r}_{\mathrm{R}}\left(\phi_{\text {guess }}, \lambda_{\text {guess }}, l_{\text {guess }}\right) ;(c \delta)_{\text {guess }}\right]$ is the guessed solution vector for the receiver Cartesian position and clock offset, with the position being dictated by the guessed WGS-84 latitude $\phi_{\text {guess }}$, longitude $\lambda_{\text {guess }}$, and altitude $l_{\text {guess }}$. The latter quantity remains fixed during this initial part of the optimization, but $\phi_{\text {guess }}$ and $\lambda_{\text {guess }}$ get updated, as does $(c \delta)_{\text {guess. }}$ Their updates are the increments that are solved for in the overdetermined linearized system of equations in Eq. (92). As with the nominal case, scaleddown increments are used, if necessary, in order to ensure that $J_{2}$ from Eq. (91) decreases for each solution increment.

\subsection{Predicted Estimation Errors}

The unknown vector $\underline{x}$ consists of a combination of (i) the unknown receiver position (ii) the receiver clock error and (iii) carrier phase bias terms. Two forms for $\underline{x}$ that correspond with two different sub-cases have been defined in Eqs. (63) and (69). Regardless of the exact form that $\underline{x}$ takes, this vector, along with the vector of unknown ionospheric parameters, $\underline{p}$, is used to define a combined error vector $\underline{\Delta}$ as follows: 


$$
\begin{aligned}
& \underline{\Delta} \triangleq\left[\begin{array}{l}
\Delta \underline{x} \\
\Delta \underline{p}
\end{array}\right] \\
& \Delta \underline{x}=\underline{x}-\underline{x}_{\text {true }} \quad ; \quad \Delta \underline{p}=\underline{p}-\underline{p}_{\text {true }}
\end{aligned}
$$

The computed set of group delays and beat carrier phase equivalent ranges, $\underline{h}(\underline{x}, \underline{p})$, is evaluated at the erroneous estimates $\underline{x}$ and $\underline{p}{ }^{2}$ It can be evaluated and related to $\underline{h}\left(\underline{x}_{\text {true }}, \underline{\underline{p}}_{\text {true }}\right)$ through a first order approximation that is performed around the true values $\underline{x}_{\text {true }}$ and $\underline{p}_{\text {true: }}$

$$
\begin{aligned}
& \underline{h}(\underline{x}, \underline{p}) \approx \underline{h}\left(\underline{x}_{\text {true }}, \underline{p}_{\text {true }}\right)+H_{x} \underline{\Delta}+H_{p} \Delta \underline{p} \\
& H_{x}=\left.\frac{\partial \underline{h}}{\partial \underline{x}}\right|_{\underline{x}_{\text {true }}, \underline{\underline{p}}_{\text {true }}} ; H_{p}=\left.\frac{\partial \underline{h}}{\partial \underline{p}}\right|_{\underline{x}_{\text {true }}, \underline{p}_{\text {true }}}
\end{aligned}
$$

The full cost function that was introduced in Eq. (79) can now be written as:

$$
\begin{aligned}
J_{1}(\underline{x}, \underline{p})= & \frac{1}{2}[\underline{y}-\underline{h}(\underline{x}, \underline{p})]^{\mathrm{T}} R^{-1}[\underline{y}-\underline{h}(\underline{x}, \underline{p})]+\frac{1}{2}(\underline{p}-\underline{\bar{p}})^{\mathrm{T}} M^{-1}(\underline{p}-\underline{\bar{p}}) \\
\approx & \frac{1}{2}\left[\underline{v}_{z}-H_{x}\left(\underline{x}-\underline{x}_{\text {true }}\right)-H_{p}\left(\underline{p}-\underline{p}_{\text {true }}\right)\right]^{T} \\
& \cdot R^{-1}\left[\underline{v}_{z}-H_{x}\left(\underline{x}-\underline{x}_{\text {true }}\right)-H_{p}\left(\underline{p}-\underline{p}_{\text {true }}\right)\right] \\
& +\frac{1}{2}\left[\underline{p}-\underline{p}_{\text {true }}-\underline{\varepsilon}_{p}\right]^{T} M^{-1}\left[\underline{p}-\underline{p}_{\text {true }}-\underline{\varepsilon}_{p}\right]
\end{aligned}
$$

Minimization of $J_{I}$ is obtained by setting all of its partial derivatives with respect the unknowns $\underline{x}$ and $\underline{p}$ to zero.

\footnotetext{
${ }^{2}$ For the remainder of this chapter, a general vector $\underline{x}$ is considered. It may take the form of $\underline{x}_{\mathrm{c}}$ or of $\underline{x}_{\mathrm{g}}$, depending on the case. The particular form chosen has no influence on any of the presented derivations.
} 


$$
\left\{\begin{aligned}
\underline{0}=\left(\frac{\partial J_{1}}{\partial \underline{x}}\right)^{T}= & -H_{x}{ }^{T} R^{-1}\left[v_{z}-H_{x}\left(\underline{x}-\underline{x}_{r u e}\right)-H_{p}\left(\underline{p}-\underline{p}_{\text {true }}\right)\right] \\
\underline{0}=\left(\frac{\partial J_{1}}{\partial \underline{p}}\right)^{T}= & -H_{p}{ }^{T} R^{-1}\left[v_{z}-H_{x}\left(\underline{x}-\underline{x}_{\text {rue }}\right)-H_{p}\left(\underline{p}-\underline{p}_{\text {true }}\right)\right] \\
& +M^{-1}\left[\underline{p}-\underline{p}_{\text {true }}-\underline{\varepsilon}_{p}\right]
\end{aligned}\right.
$$

The solution is

$$
\left[\begin{array}{c}
\underline{\hat{x}} \\
\hat{\hat{p}}
\end{array}\right]=S_{1}^{-1}\left[\begin{array}{c}
H_{x}{ }^{T} R^{-1} \underline{v}_{z} \\
H_{p}{ }^{T} R^{-1} \underline{v}_{z}+M^{-1} \underline{\varepsilon}_{p}
\end{array}\right]+\left[\begin{array}{c}
\underline{x}_{t r u e} \\
\underline{p}_{\text {true }}
\end{array}\right]
$$

where

$$
S_{1} \triangleq\left[\begin{array}{cc}
H_{x}^{T} R^{-1} H_{x} & H_{x}^{T} R^{-1} H_{p} \\
H_{p}^{T} R^{-1} H_{x} & H_{p}{ }^{T} R^{-1} H_{p}+M^{-1}
\end{array}\right]
$$

and the resulting predicted estimation error takes the form

$$
\underline{\Delta}_{P} \triangleq\left[\begin{array}{l}
\underline{\hat{x}}-\underline{x}_{\text {true }} \\
\hat{\hat{p}}-\underline{p}_{\text {true }}
\end{array}\right]=S_{1}^{-1}\left[\begin{array}{c}
H_{x}{ }^{T} \\
H_{p}{ }^{T}
\end{array}\right] R^{-1} \underline{v}_{z}+S_{1}^{-1}\left[\begin{array}{c}
0_{N_{x} x N_{p}} \\
M^{-1}
\end{array}\right] \underline{\varepsilon}_{p}
$$

where $N_{x}$ is the number of elements in $\underline{x}$. The separate, independent contributions of the two different sets of errors, $\underline{v}_{z}$ and $\underline{\varepsilon} p$, are distinctly evident. It is worth noting that for the case of a zero mean Gaussian distributed $\underline{\varepsilon} p$, the estimation error $\underline{\Delta} \mathrm{P}$ is unbiased according to the linearized model of Eq. (99). 


\subsection{The Mean Square Error Matrix and the $\boldsymbol{A}$ Posteriori Covariance}

Two tools for evaluating the performance for the proposed system are the mean square error matrix and the a posteriori covariance matrix for the errors in the estimates of the vectors $\underline{x}$ and $\underline{p}$. Two different cases are considered. The first case assumes uncertainty for both $\underline{x}$ and $\underline{p}$. In the second case, ionospheric modeling errors are modeled as constants rather than random samples.

\subsubsection{The case of random ionosphere parameters errors}

The associated estimation mean square error matrix can be computed based on the computed linearized approximate error. If $\underline{\varepsilon}_{p}$ is sampled from a Gaussian distribution that satisfies $E\left[\underline{\varepsilon}_{p}\right]=\underline{0}$ and $E\left[\underline{\varepsilon}_{p} \underline{\varepsilon}_{p}^{\mathrm{T}}\right]=M$, then the estimation error covariance matrix is given by the standard form

$$
\begin{aligned}
\Sigma_{1} & \triangleq E\left[\left[\begin{array}{l}
\hat{\hat{x}}-\underline{x}_{\text {true }} \\
\hat{\hat{p}}-\underline{p}_{\text {true }}
\end{array}\right]\left[\begin{array}{l}
\underline{\hat{x}}-\underline{x}_{\text {true }} \\
\hat{\hat{p}}-\underline{p}_{\text {true }}
\end{array}\right]^{T}\right]= \\
& =S_{1}^{-1}\left[\left[\begin{array}{c}
H_{x}{ }^{T} \\
H_{p}{ }^{T}
\end{array}\right] R^{-1}\left[\begin{array}{ll}
H_{x} & H_{p}
\end{array}\right]+\left[\begin{array}{cc}
0 & 0 \\
0 & M^{-1}
\end{array}\right]\right] S_{1}^{-T}=S_{1}^{-1}
\end{aligned}
$$

While this sort of computation may provide a certain understanding of the system's anticipated performance, its most distinct weakness is the limited information that such analysis would provide about how errors are affected by particular a priori ionospheric model errors, or, more precisely, how positioning and timing errors might be related to the magnitude of $\xi$, as defined in Eq. (24). Such observations can be made with test cases of different $\xi$ values that are kept constant. This is the case of a constant $\underline{\varepsilon}_{p}$. The analysis 
given in Eq. (100) only gives an approximation of the expected statistical impact of errors in the a priori ionosphere.

\subsubsection{The case of constant $a$ priori ionosphere parameters errors}

An important case for error analysis is the case of constant $\varepsilon_{p}$. The scenarios included in this analysis consist of cases for which the errors in the a priori ionosphere model are kept constant, with different characterizing $\xi$ values that are significant to the discussion that follows. The mean square error matrix in this case takes the form

$$
\tilde{\Sigma}_{2}=S_{1}^{-1}\left[\left[\begin{array}{c}
H_{x}{ }^{T} \\
H_{p}{ }^{T}
\end{array}\right] R^{-1}\left[\begin{array}{ll}
H_{x} & H_{p}
\end{array}\right]+\left[\begin{array}{cc}
0 & 0 \\
0 & M^{-1} \underline{\varepsilon}_{p} \underline{\varepsilon}_{p}^{T} M^{-1}
\end{array}\right]\right] S_{1}^{-T}
$$

The right-most term in brackets embodies the contribution of the error bias that arises from the constant a priori ionosphere model error. This term should be omitted when one is interested in evaluating the distribution of position errors about their mean. The result constitutes the covariance matrix for the errors in the estimates of the vectors $\underline{x}$ and $\underline{p}$ about this mean:

$$
\Sigma_{2} \triangleq E\left[\left[\begin{array}{l}
\underline{\hat{x}}-\underline{x}_{\text {true }} \\
\underline{\hat{p}}-\underline{p}_{\text {true }}
\end{array}\right]\left[\begin{array}{c}
\underline{\hat{x}}-\underline{x}_{\text {true }} \\
\hat{\hat{p}}-\underline{p}_{\text {true }}
\end{array}\right]^{T}\right]=S_{1}^{-1}\left[\left[\begin{array}{c}
H_{x}{ }^{T} \\
H_{p}{ }^{T}
\end{array}\right] R^{-1}\left[\begin{array}{ll}
H_{x} & H_{p}
\end{array}\right]\right] S_{1}^{-T}
$$

\subsubsection{Ionosphere model covariance mismatch}

This subsection considers the case where the matrix that is used in the optimization process computation, $M_{\mathfrak{c}}$, is different from the matrix $M$ that truly represents the 
correlation between the various ionosphere parameters. The cost function of equation (95) takes the following form in this case:

$$
\begin{aligned}
J_{1, M}(\underline{x}, \underline{p})= & \frac{1}{2}[\underline{P}-\underline{h}(\underline{x}, \underline{p})]^{\mathrm{T}} R^{-1}[\underline{P}-\underline{h}(\underline{x}, \underline{p})]+\frac{1}{2}(\underline{p}-\underline{\bar{p}})^{\mathrm{T}} M_{c}^{-1}(\underline{p}-\underline{\bar{p}}) \\
\approx & \frac{1}{2}\left[\underline{v}_{z}-H_{x}\left(\underline{x}-\underline{x}_{\text {true }}\right)-H_{p}\left(\underline{p}-\underline{p}_{\text {true }}\right)\right]^{T} \\
& \cdot R^{-1}\left[\underline{v}_{z}-H_{x}\left(\underline{x}-\underline{x}_{\text {true }}\right)-H_{p}\left(\underline{p}-\underline{p}_{\text {true }}\right)\right] \\
& +\frac{1}{2}\left[\underline{p}-\underline{p}_{\text {true }}-\underline{\varepsilon}_{p}\right]^{T} M_{c}^{-1}\left[\underline{p}-\underline{p}_{\text {true }}-\underline{\varepsilon}_{p}\right]
\end{aligned}
$$

The estimation error is

$$
\begin{aligned}
& \underline{\Delta}_{M} \triangleq\left[\begin{array}{c}
\underline{\hat{x}}-\underline{x}_{\text {true }} \\
\hat{\hat{p}}-\underline{p}_{\text {true }}
\end{array}\right]_{M_{c}}=S_{1, M}{ }^{-1}\left[\begin{array}{c}
H_{x}{ }^{T} \\
H_{p}{ }^{T}
\end{array}\right] R^{-1} \underline{v}_{z}+S_{1, M}{ }^{-1}\left[\begin{array}{c}
0_{4 x N_{p}} \\
M_{c}{ }^{-1}
\end{array}\right] \underline{\varepsilon}_{p} \\
& S_{1, M} \triangleq\left[\begin{array}{cc}
H_{x}{ }^{T} R^{-1} H_{x} & H_{x}{ }^{T} R^{-1} H_{p} \\
H_{p}{ }^{T} R^{-1} H_{x} & H_{p}{ }^{T} R^{-1} H_{p}+M_{c}{ }^{-1}
\end{array}\right]
\end{aligned}
$$

and the corresponding mean square error matrix becomes

$$
\begin{aligned}
\tilde{\Sigma}_{3} & \triangleq E\left[\left[\begin{array}{c}
\underline{\hat{x}}-\underline{x}_{\text {true }} \\
\underline{\hat{p}}-\underline{p}_{\text {true }}
\end{array}\right]\left[\begin{array}{l}
\underline{\hat{x}}-\underline{x}_{\text {true }} \\
\hat{\hat{p}}-\underline{p}_{\text {true }}
\end{array}\right]^{T}\right] \\
& =S_{1, M}{ }^{-1}\left[\left[\begin{array}{c}
H_{x}{ }^{T} \\
H_{p}{ }^{T}
\end{array}\right] R^{-1}\left[\begin{array}{ll}
H_{x} & H_{p}
\end{array}\right]+\left[\begin{array}{cc}
0 & 0 \\
0 & M_{c}{ }^{-1} M M_{c}{ }^{-1}
\end{array}\right]\right] S_{1, M}{ }^{-T}
\end{aligned}
$$

\subsubsection{Measurement noise covariance mismatch}

When the measurement noise covariance matrix that is used in computations, $R_{\mathrm{c}}$, is different from the $R$ matrix that holds values for true measurement noise, the cost function that is used with the algorithm takes the form 


$$
\begin{aligned}
J_{1, R}(\underline{x}, \underline{p})= & \frac{1}{2}[\underline{P}-\underline{h}(\underline{x}, \underline{p})]^{\mathrm{T}} R_{c}^{-1}[\underline{P}-\underline{h}(\underline{x}, \underline{p})]+\frac{1}{2}(\underline{p}-\underline{\bar{p}})^{\mathrm{T}} M^{-1}(\underline{p}-\underline{\bar{p}}) \\
\approx & \frac{1}{2}\left[\underline{v}_{z}-H_{x}\left(\underline{x}-\underline{x}_{\text {true }}\right)-H_{p}\left(\underline{p}-\underline{p}_{\text {true }}\right)\right]^{T} \\
& \cdot R_{c}^{-1}\left[\underline{v}_{z}-H_{x}\left(\underline{x}-\underline{x}_{\text {true }}\right)-H_{p}\left(\underline{p}-\underline{p}_{\text {true }}\right)\right] \\
& +\frac{1}{2}\left[\underline{p}-\underline{p}_{\text {true }}-\underline{\varepsilon}_{p}\right]^{T} M^{-1}\left[\underline{p}-\underline{p}_{\text {true }}-\underline{\varepsilon}_{p}\right]
\end{aligned}
$$

The predicted estimate error becomes

$$
\begin{aligned}
& \underline{\Delta}_{R} \triangleq\left[\begin{array}{c}
\underline{\hat{x}}-\underline{x}_{\text {true }} \\
\hat{\hat{p}}-\underline{p}_{\text {true }}
\end{array}\right]_{R_{c}}=S_{1, R}{ }^{-1}\left[\begin{array}{c}
H_{x}{ }^{T} \\
H_{p}{ }^{T}
\end{array}\right] R_{c}^{-1} \underline{v}_{z}+S_{1, R}{ }^{-1}\left[\begin{array}{c}
0_{4 x N_{p}} \\
M^{-1}
\end{array}\right] \underline{\varepsilon}_{p} \\
& S_{1, R} \triangleq\left[\begin{array}{cc}
H_{x}{ }^{T} R_{c}{ }^{-1} H_{x} & H_{x}{ }^{T} R_{c}{ }^{-1} H_{p} \\
H_{p}{ }^{T} R_{c}{ }^{-1} H_{x} & H_{p}{ }^{T} R_{c}{ }^{-1} H_{p}+M^{-1}
\end{array}\right]
\end{aligned}
$$

Assuming a constant $\underline{\varepsilon}$ as in Subsection 4.4.2, the corresponding mean square error matrix is

$$
\begin{aligned}
\tilde{\Sigma}_{4} \triangleq E\left[\left[\begin{array}{l}
\hat{\hat{x}}-\underline{x}_{\text {true }} \\
\hat{\hat{p}}-\underline{p}_{\text {true }}
\end{array}\right]\left[\begin{array}{l}
\hat{\hat{x}}-\underline{x}_{\text {true }} \\
\hat{\hat{p}}-\underline{p}_{\text {true }}
\end{array}\right]^{T}\right] \\
\quad=S_{1, R}{ }^{-1}\left[\left[\begin{array}{l}
H_{x}{ }^{T} \\
H_{p}{ }^{T}
\end{array}\right] R^{-1}\left[\begin{array}{ll}
H_{x} & H_{p}
\end{array}\right]+\left[\begin{array}{cc}
0 & 0 \\
0 & M^{-1} \underline{\varepsilon}_{p} \underline{\varepsilon}_{p}{ }^{T} M^{-1}
\end{array}\right]\right] S_{1, R}{ }^{-T}
\end{aligned}
$$

\subsubsection{The horizontal and vertical position error ellipses}

A useful tool in positioning performance analysis is the error ellipse that is a twodimensional confidence region. Let $\Delta_{\mathrm{E}}$ and $\Delta_{\mathrm{N}}$ denote the position error random variables in an east-north reference frame (EN). The combined horizontal error covariance matrix is given by 


$$
\Sigma_{E N} \triangleq E\left[\left[\begin{array}{c}
\Delta_{E}-\bar{\Delta}_{E} \\
\Delta_{N}-\bar{\Delta}_{N}
\end{array}\right]\left[\begin{array}{c}
\Delta_{E}-\bar{\Delta}_{E} \\
\Delta_{N}-\bar{\Delta}_{N}
\end{array}\right]^{T}\right]
$$

where $\bar{\Delta}_{\mathrm{E}}$ and $\bar{\Delta}_{\mathrm{N}}$ designate the position error variables' expected values. Let $d_{1}$ and $d_{2}$ denote $\Sigma_{\mathrm{EN}}$ 's eigenvalues and let $v_{1}$ and $v_{2}$ denote $\Sigma_{\mathrm{EN}}$ 's corresponding eigenvectors. A new reference frame $F$ is defined such that its origin is located at $\left(\bar{\Delta}_{\mathrm{E}}, \bar{\Delta}_{\mathrm{N}}\right)$ and whose axes are aligned with $v_{1}$ and $v_{2}$. A corresponding coordinates system $\left(\Delta_{1}, \Delta_{2}\right)$ where distances are measured along $F$ 's axes is defined as well. The random variable

$$
s=\frac{\Delta_{1}^{2}}{d_{1}}+\frac{\Delta_{2}^{2}}{d_{2}}
$$

has Chi Square distribution of degree 2, then $p(s<4.605)=0.90$ defines an ellipse that is guaranteed to contain $90 \%$ of the plotted errors [35]. This ellipse's major and minor axes are aligned with $F$ and their lengths are $2 \sqrt{4.605 d_{1}}$ and $2 \sqrt{4.605 d_{2}}$. The vertical $90 \%$ error ellipse that considers errors in the east-up (EU) plane is computed in the same way.

Figure 6 illustrates the two reference frames and the 90\% error ellipse for an arbitrary set of position east/north error pairs.

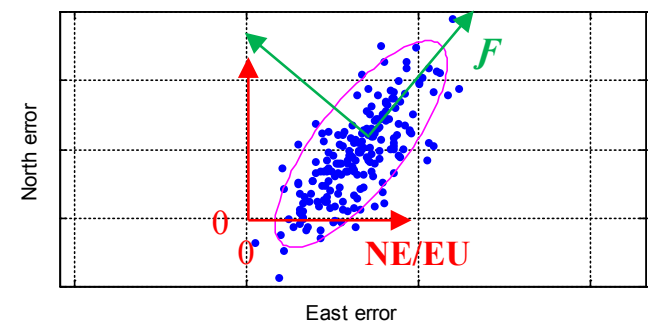

Figure 6: Illustration of: the NE or EU reference frame, the $f$ reference frame, the $90 \%$ error ellipse and its two axes. 


\section{CHAPTER 5}

\section{A SIMPLIFIED MODEL-BASED ESTIMATOR}

Previous chapters presented the most recent phase of this study that includes an advanced physical model for propagating signals and an optimal batch filter that has been developed to solve the resulting optimization problem. Later chapters will present analyses of filter algorithm performance and behavior. It is presumed that the use of advanced models for propagating signals gives a reasonable level of fidelity to that analysis that cannot be achieved with very simplified models.

However, the discussion will not be complete without a review of a second, simplified model that has been used in earlier phases of this study. The simplified signal propagation model, and an associated batch filter that addresses its unique characteristics, were first introduced and discussed in Ref. [5]. That paper presented preliminary results for the HF navigation concept. Moreover, it should be noted that the simplified model, along with many of the associated filter's components that have been developed, are practically used with the current algorithm's ray-path solver, as described in Subsection 3.4.2.

This chapter will cover the simplified models' physical and mathematical components. It will highlight the differences between the simplified filter and the filter that is the main subject of this dissertation, and it will list unique characteristics for the former. 


\subsection{Physical and Mathematical Models}

\subsubsection{Earth and the ionosphere}

The simplified model shares the same Earth and ionosphere models that are used in the advanced model, except the effect of Earth's magnetic field is not considered. The most significant difference between the two models lies in the manner at which signals travel through the ionosphere, and in particular, in the nature of signals' trajectories and ionospheric bounces. In the simplified model, HF signals are assumed to reflect off of the ionosphere in a specular manner. A reflection is assumed to occur when the angle of incidence and the local index of refraction $n_{\text {simp }}$ satisfy a condition of total internal reflection. Using Snell's law, the internal reflection condition is satisfied when the angle of a refracted signal would be $90^{\circ}$. The condition used here incorporates an approximation for the vertical variations of $n_{\text {simp }}$ that assumes it to equal 1 below the reflection point and to transition abruptly to its value dictated by $N_{e}(\underline{r})$ at the reflection point. Let $\psi_{0}$ be the angle of incidence as measured relative to the local unit normal vector of the local constant electron density surface

$$
\underline{u}_{s i m p}=-\nabla N_{e}(\underline{r}) /\left\|N_{e}(\underline{r})\right\|
$$

Then these assumptions lead to the following version of Snell's law for the reflection condition:

$$
1 \cdot \sin \left(\psi_{0}\right)=n_{\text {simp }} \cdot \sin \left(90^{\circ}\right)=n_{\text {simp }}
$$

At a reflection point, the phase index of refraction depends on electron density $N_{e}(\underline{r})$ and on the signal's frequency $\omega$. This dependency takes the form: 


$$
n_{\text {simp }}(\underline{r})=\sqrt{1-C_{1} N_{e}(\underline{r}) / \omega^{2}} \quad, \quad C_{1}=3182.73849408628
$$

where $N_{e}(\underline{r})$ is given in units of electrons $/ \mathrm{m}^{3}$ and $\omega$ is given in units of radians $/ \mathrm{sec}$. This simplified refractive index model ignores the effects of the Earth's magnetic field.

\subsubsection{Bounce points, ray-paths, and the measurement model}

Unlike with the full model for which signals' speeds vary, ray-hops are curved, and ionospheric refractions/reflections are calculated with a raytracing engine, the ray-paths of this simplified model are ordered sequences of straight line segments. Each line segment is defined by its two end points. For a connected ray path of $m$ line segments, $m+1$ points are defined as follows: The first point is the known location of the transmitter, $q$. The second point is a bounce point located on the ionosphere surface. All other bounce points are alternately located on the Earth and the ionosphere. The last point is the position of the receiver, $\underline{r}_{\mathrm{R}}$. As with the full model, bounce points are defined in the ECEF Cartesian coordinate system. The vector of unknown ECEF X-Y-Z coordinates for the $j^{\text {th }}$ ray path's bounce points, takes the form

$$
\underline{\eta}_{s i m p, j}=\left[X_{1}, X_{2}, \ldots, X_{m-1}, Y_{1}, Y_{2}, \ldots, Y_{m-1}, Z_{1}, Z_{2}, \ldots, Z_{m-1}\right]^{T}
$$

and consists of coordinates for two types of bounce points. Odd-indexed X-Y-Z trios describe the position of ionosphere bounce points, while even-indexed X-Y-Z trios describe the position of Earth bounce points. Note that the former type of bounce point does not exist with the advanced signal propagation model. 
Let $\rho_{\text {simp, } \mathrm{j}}$ be the true length of the $j^{\text {th }}$ ray-path. Let $y_{\text {simp, } \mathrm{j}}$ be the measured pseudorange of that ray-path, which equals the speed of light $c$ multiplied by the difference between the measured reception time according to the erroneous receiver clock and the true transmission time according to a calibrated beacon transmitter clock. The $j^{\text {th }}$ measurement equation can be written as

$$
\begin{aligned}
y_{\text {simp }, j} & =h_{\text {simp }, j}\left(\underline{x}_{g}, \underline{\hat{p}}_{j}\right)+v_{g, j}=\breve{h}_{\text {simp }, j}\left(\underline{r}_{R}, \underline{\hat{p}}_{j}\right)+c \delta+v_{g, j} \\
& =\tilde{h}_{\text {simp }, j}\left[\underline{r}_{R}, \underline{\eta}_{\text {simp }, j}\left(\underline{r}_{R}, \underline{\hat{p}}_{j}\right)\right]+c \delta+v_{g, j}
\end{aligned}
$$

where $\breve{h}_{\text {simp, j }}$ and $\tilde{h}_{\text {simp, j }}$ are alternate forms of the model for $\rho_{\text {simp, } \mathrm{j}}$.

Equations (62) and (115) that describe the group delay measurement equations for the advanced and simplified models, respectively, may appear similar. However, the two equations differ in significant ways. The scalar function $\tilde{h}_{\mathrm{g}, \mathrm{j}}$ computes the range equivalent group delay for a propagating dispersive signal, that depends on $\hat{p}_{\mathrm{j}}$ directly, as well as indirectly through $\eta_{\text {simp.j. }}$. In contrast, $\tilde{h}_{\text {simp,j }}$ returns the total Euclidian length of a segmented ray-path, that does not depend directly on $\hat{p}_{j}$. Moreover, the implicit functions $\underline{\eta}_{\mathrm{j}}\left(\underline{\underline{r}}_{\mathrm{R}}, \hat{\underline{p}}_{\mathrm{j}}\right)$ and $\underline{\eta}_{\mathrm{simp}} \mathrm{j}\left(\underline{\underline{r}}_{\mathrm{R}}, \hat{\underline{p}}_{\mathrm{j}}\right)$ take different forms for the two models.

\subsubsection{Bounce point equations}

As described in the previous Subsection, the simplified ray-path model assumes bounce points on both the Earth's and the ionosphere's "surfaces". For Earth surface bounce points, the same set of equations holds true as is given in Subsection 3.2.2 for the advanced model. 
For ionosphere bounce points, Type-B and Type-C equations take the same form as Earth bounce points, except the surface normal vector defined in Eq. (111) must be used in place of the surface normal defined in Eq. (34). However, Type-A equations take a different form that originates from the different reflection condition that is considered for the simplified model. Namely, for the $k^{\text {th }}$ bounce point, the modified Type-A reflection condition equation can be written as

$$
\begin{aligned}
& 0=g_{A_{s i m p}, k}\left(\underline{v}_{f, k}, \underline{v}_{0, k}, \underline{\eta}_{\text {simp }, k}, \underline{u}_{s i m p, k}\right) \\
&=\left(\underline{v}_{f, k}{ }^{T} \underline{u}_{s i m p, k}\right)^{2}-\frac{N_{e}\left(\underline{\eta}_{\text {simp }, k}\right)}{\omega^{2}} C_{1}\left(\underline{v}_{f, k}{ }^{T} \underline{v}_{f, k}\right)\left(\underline{u}_{\text {simp }, k}{ }^{T} \underline{u}_{\text {simp }, k}\right)
\end{aligned}
$$

The direction vector $\underline{v}_{\mathrm{f}}$ is computed by differencing line segment end points that are found in $\underline{r}_{\mathrm{R}}$, $\underline{q}$, or $\underline{\eta}_{s i m p, j}$. Reference [5] demonstrates how this equation is derived from Eqs. (112) and (113) of this dissertation.

\subsubsection{Solving for the bounce points $\underline{\eta}_{\text {simp }}$}

A solution for the unknown location of Earth and ionospheric bounce points is obtained through a minimization of the cost function

$$
J_{R P, s i m p}\left(\underline{\eta}_{\text {simp }, m}\right)=\frac{1}{2} \underline{\tilde{g}}_{\text {simp }, m}\left(\underline{\eta}_{\text {simp }, m}, \underline{r}_{R}, \underline{p}\right)^{T} \underline{\tilde{g}}_{\text {simp }, m}\left(\underline{\eta}_{\text {simp }, m}, \underline{r}_{R}, \underline{p}\right)
$$

using a process which is similar to that of the full-model which was described in Section 3.4.1. The minimization is with respect to $\eta_{\text {simp,m. }} \tilde{g}_{\text {simp,m }}$ denotes the Eq. (32)-type formulation of the simplified-model bounce points equations. Minimization is performed using a method that is similar to that of the full-model, except that with the simplified 
model, the sensitivities of bounce points equations with respect to their position are obtained analytically.

An initial guess for $\eta_{\text {simp,m }}$ is generated using a thin-shell, latitude/longitude heightdependent ionosphere model. The height for this bi-quintic splined modeled thin-shell is the height for which electron density reaches $80 \%$ of its maximal density. This initial guess is generated based on the assumptions that:

a) The set of a ray-path's bounce points (both Earth and ionospheric) lies in a plane that contains the great circle that is defined by that ray-path's start and end points

b) The distance between adjacent Earth bounce points is proportional to the height of the thin shell, measured at the mean-point between the two bounce points

Additional initial guesses are generated in a similar way, where shells with fixed heights above the WGS- 84 are considered instead of the $80 \%$ shell that has been defined above. 


\subsection{A Batch Filter for the Simplified Model}

Since the fundamental optimization problem that is addressed with the different batch filters that are used in this study is independent of ray-path models, the earlier form of the batch estimation cost function is very similar to that of Eq. (79). It takes the form

$$
\begin{gathered}
J_{1, \operatorname{simp}}\left(\underline{x}_{g}, \underline{p}\right)=\frac{1}{2}\left[\underline{y}_{\text {simp }}-\underline{h}_{\text {simp }}\left(\underline{x}_{g}, \underline{p}\right)\right]^{\mathrm{T}} R^{-1}\left[\underline{y}-\underline{h}_{\operatorname{sim} p}\left(\underline{x}_{g}, \underline{p}\right)\right] \\
+\frac{1}{2}(\underline{p}-\underline{\bar{p}})^{\mathrm{T}} M^{-1}(\underline{p}-\underline{\bar{p}})
\end{gathered}
$$

It omits the $\zeta$ scaling factor which is used with the advanced estimator. $y_{\text {simp }}$ consists of group delay measurements exclusively. Therefore it is an $N \times 1$ stacked vector, and $R$ is an $N \mathrm{x} N$ measurement error covariance matrix. As with the advanced filter, a modification of the cost function is used when the position solution appears to be far from convergence. It takes the same form of Eq. (91) except that the $\underline{h}_{\mathrm{g}}$ terms in that equation are replaced by $\underline{h}_{\text {simp }}$, and only the latitude, longitude, and clock offset of the user receiver are optimized. The altitude and the ionosphere parameters are held constant.

\subsubsection{The Modified Gauss-Newton Method}

The top-level procedure for the Gauss-Newton method that solves the optimization problem for the simplified ray-path problem is similar to the procedure that has been described in Subsection 4.2.1. Namely, it consists of the same sequence of steps: linearization, determination of a descent direction using matrix methods, and determination of a step size scaling parameter that yields reduction in the cost function. It also includes the measurements exclusion feature. It does not, however, include a measurements rejection mechanism. 


\subsubsection{Measurement Model Sensitivity Matrices}

The simplified model signal propagation directions are determined directly by the location of the bounce points. The bounce point locations are dependent on the position of the receiver and on the values that the ionosphere parameters take in the vicinity of a ray-path. Therefore, the variant of Eq. (32) that applies to the simplified filter takes the form:

$$
\underline{0}=\underline{\tilde{g}}_{\text {simp }, k}\left(\underline{\eta}_{\text {simp }, j}\left[\underline{r}_{R}, \underline{\hat{p}}_{j}\right], \underline{r}_{R}, \underline{\hat{p}}_{j}\right)
$$

Differentiation of this equation with respect $\underline{r}_{\mathrm{R}}$ yields and solution for the bounce point partial derivatives with respect to $\underline{r}_{\mathrm{R}}$ yields:

$$
\frac{\partial \underline{\eta}_{s i m p, j}}{\partial \underline{r}_{R}}=-\left[\frac{\partial \underline{\tilde{g}}_{\text {simp }, j}}{\partial \underline{\eta}_{\text {simp }, j}}\right]^{-1} \frac{\partial \underline{\tilde{g}}_{\text {simp }, j}}{\partial \underline{r}_{R}}
$$

where $\tilde{g}_{\text {simp }, \mathrm{j}}$ consists of all bounce point equations that apply for the $j^{\text {th }}$ ray-path. The matrix of partial derivative sensitivities of the $j^{\text {th }}$ measurement model pseudorange to changes in the estimate of the receiver's position can be written as:

$$
\begin{aligned}
\frac{\partial \underline{h}_{\text {simp }, j}}{\partial \underline{r}_{R}}= & \frac{\partial \underline{\tilde{h}}_{\text {simp }, j}}{\partial \underline{\eta}_{\text {simp }, j}} \frac{\partial \underline{\eta}_{\text {simp }, j}}{\partial \underline{r}_{R}}+\frac{\partial \underline{\tilde{h}}_{\text {simp }, j}}{\partial \underline{r}_{R}}= \\
& -\frac{\partial \underline{\tilde{h}}_{s i m p, j}}{\partial \underline{\eta}_{\text {simp }, j}}\left[\frac{\partial \underline{\tilde{g}}_{s i m p, j}}{\partial \underline{\eta}_{\text {simp }, j}}\right]^{-1} \frac{\partial \underline{\tilde{g}}_{\text {simp }, j}}{\partial \underline{r}_{R}}+\frac{\partial \underline{\tilde{h}}_{s i m p, j}}{\partial \underline{r}_{R}}
\end{aligned}
$$

Similarly, taking the partial derivative of the $\tilde{g}_{\text {simp }}$ equation with respect to $\hat{p}_{\mathrm{j}}$ and solving the bounce point partial derivatives with respect to $\hat{p}_{\mathrm{j}}$ yields: 


$$
\frac{\partial \underline{\eta}_{s i m p, j}}{\partial \underline{p}_{j}}=-\left[\frac{\partial \underline{\tilde{g}}_{s i m p, j}}{\partial \underline{\eta}_{s i m p, j}}\right]^{-1} \frac{\partial \underline{\tilde{g}}_{s i m p, j}}{\partial \underline{p}_{j}}
$$

The partial derivative the $j^{\text {th }}$ modeled pseudorange with respect to changes in the estimates of the ionospheric parameters is

$$
\frac{\partial \underline{h}_{\text {simp }, j}}{\partial \underline{\hat{p}}_{j}}=\frac{\partial \underline{\tilde{h}}_{\text {simp }, j}}{\partial \underline{\eta}_{\text {simp }, j}} \frac{\partial \underline{\eta}_{\text {simp }, j}}{\partial \underline{p}_{j}}+\underbrace{\frac{\partial \tilde{\hat{h}}_{\text {simp }, j}}{\partial \underline{p}_{j}}}_{0}=-\frac{\partial \underline{\tilde{h}}_{\text {simp }, j}}{\partial \underline{\eta}_{\text {simp }, j}}\left[\frac{\partial \underline{\tilde{g}}_{\text {simp }, j}}{\partial \underline{\eta}_{\text {simp }, j}}\right]^{-1} \frac{\partial \underline{\tilde{g}}_{\text {simp }, j}}{\partial \underline{p}_{j}}
$$

It should be noted how the above sets of measurement sensitivity matrices are very different from their advanced-model equivalents, whose formulation and derivation are presented in Subsection 3.5.3.

\subsubsection{Calculation of Jacobian matrices}

The sensitivity matrices computing procedure for the simplified model's batch filter shows some resemblance to the procedure that is performed for the full model. The partial derivatives of $\underline{h}_{\mathrm{simp}}$ with respect $\underline{\eta}_{\mathrm{j}}$ and $\underline{\underline{R}}_{\mathrm{R}}$ are straightforward due to the simplistic nature of $\underline{h}_{\text {simp. }}$. The manner in which the $\tilde{g}_{\text {simp }}$ bounce-points equations depend on $\underline{r}_{\mathrm{R}}$ makes the computation of $\partial \tilde{g}_{\text {simp }} / \partial \underline{r}_{\mathrm{R}}$ somewhat more involved. However, computation of $\partial \tilde{g}_{\text {simp }} / \partial \hat{\underline{p}}_{\mathrm{j}}$ is complicated and tends to be demanding in computation time. Finally, special care should be taken with the Jacobian $\partial \tilde{g}_{\text {simp }} / \partial \underline{\eta}_{\text {simp,j. }}$ The details of this computation are presented in Appendix B. 


\subsection{Performance}

A complete description of a limited performance analysis that has been conducted with the simplified ray-paths model was presented in Ref. [5]. This analysis's highlights are additionally reviewed later in Section 7.1. 


\section{CHAPTER 6}

\section{ALGORITHM OPERATION AND VALIDATION}

This chapter discusses various aspects of implementing the concepts and mathematical derivations that have been presented in the previous chapters, in the form of a digital simulation that is used to assess the developed batch filtering algorithm. This truth-model simulation is presented next, in the first section. Batch filtering algorithm functionality and limitations, including solution convergence, is the subject of following sections. The chapter concludes with a simulation-based validation for the different forms of filter statistical analyses that have been derived.

\subsection{Truth-Model Simulation and Batch Filter Monitoring}

A MATLAB ${ }^{\circledR}$ truth-model simulation has been developed for algorithm validation, algorithm assessment, and performance analysis. General comments on implementation, coding concepts, and hardware are included in Appendix C.

The simulation enables testing of any desired combination of ground station array, ray-path characteristics, measurement error models, ionosphere error models, and other parameters. As discussed earlier, it has been shown that not all such combinations are

physically feasible. Feasibility of a given configuration is tested during the first stage of simulation execution. 
Figure 7 illustrates a typical simulation setup, showing the different curved signals' ray-paths, starting from ground stations and eventually arriving at the UE. Different raypaths which are transmitted from the same ground station are shown in different shades of green, with gray circles denoting their ground bounce-points. The blue circles in the figure denote ground stations, with the corresponding broadcast signals' identifying indices shown next to the circles. The magenta diamonds with adjacent three digit numbers denote ionosphere model bi-quintic spline grid nodes, with their identifying numbers next to them. The North American coastline is shown as a thin blue line. The dashed red line illustrates the Gauss-Newton algorithm's convergence performance. It plots a history of successive receiver position solution guesses. The receiver's true location is marked with a black, thin X.

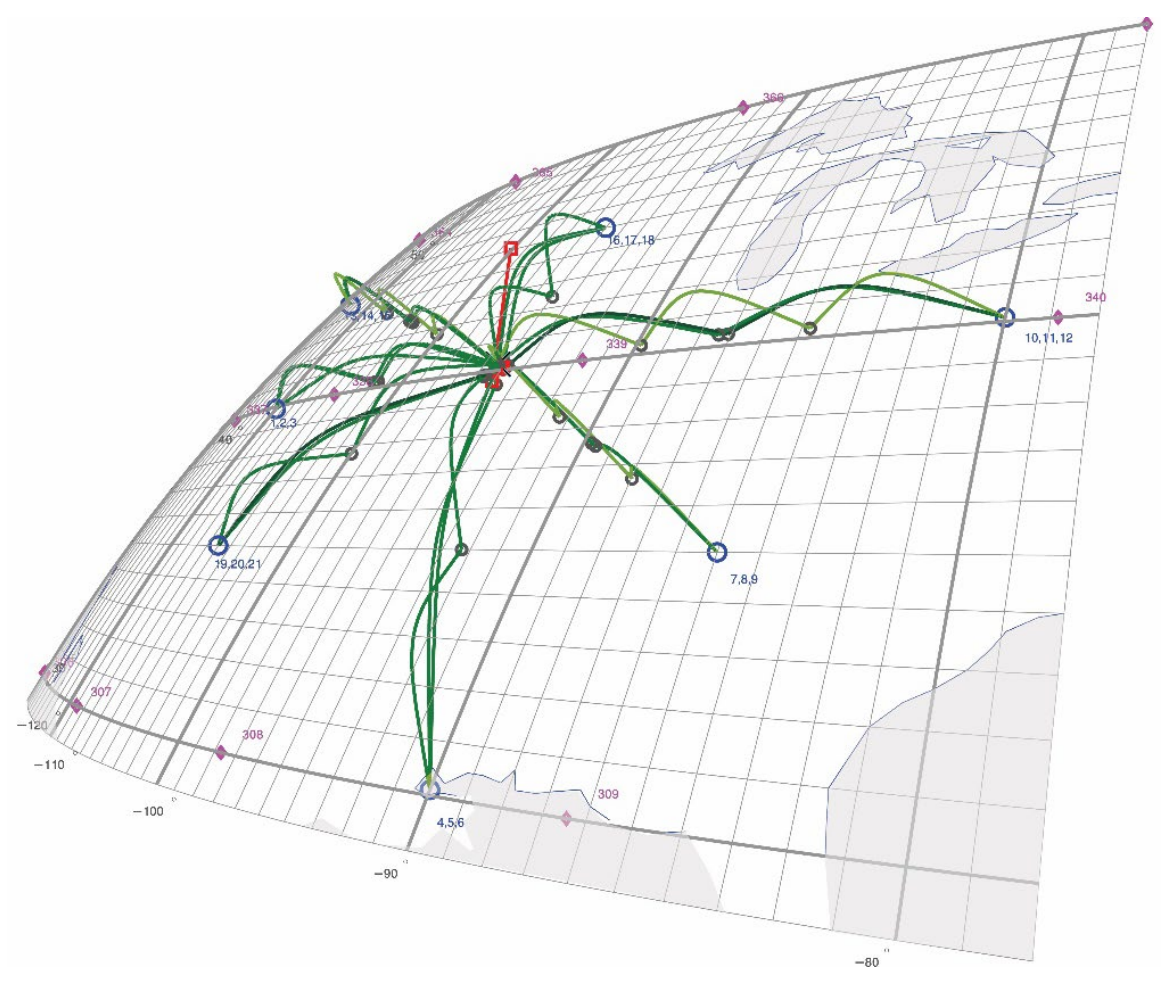

Figure 7: A typical test case setup used with the truth-model simulation. 
The duration of the batch filter execution for a single test case in an execution mode known as Nominal Scenario Mode is usually on the order of hours. Consequently, filter performance simulations are closely monitored during their execution. The monitoring screen, shown in Fig. 8, presents data about progress of the solution process. The upper screen presents the results for the sub-processes that are conducted for every ray-hop of all ray-paths at all process steps. These steps include all top-level Gauss-Newton iterations and their intermediate step-size factor $\alpha$ determination sub-processes. Table rows designated $<G N>$ are associated with Gauss-Newton iterations. The number on the right indicates iteration number. Table rows designated alpha are associated with stepsize determination steps for those Gauss-Newton iterations. The numbers on the right indicate halving-counts, so that alpha 1 indicates no halving at all, alpha 2 indicates one halving, and so on. Each row in this plot, either a Gauss-Newton iteration or one of its halving steps, is regarded as a numbered process step. Columns are associated with raypaths and ray-hops. For instance, the column labeled 9.3 presents ray-hop computation results for the third ray-hop of the ninth ray-path. A green cell with a ' 0 ' indicates a success in a ray-hop solving attempt. A red cell indicates a failure, where the accompanying number provides information on the nature of that failure. Gray cells indicate unprocessed ray-hops. This may be the case when preceding ray-hop solving attempts for that ray-path have failed. It should be noted that this table does not provide detailed information on ray-path solving inner processes expect for their final outcome which is reported using color/number code as mentioned above. 
The circular plot in the bottom left corner of the screen presents the sequence of horizontal position errors in a plane that has an east/west axis and a south/north axis. This sequence starts at the point that is marked ' 1 ', which is the error for the position a priori guess. The radial scaling for this polar-type plot is logarithmic. Other panels provide information on how clock offset estimation, the Ionosphere Error Index $-\xi$, carrier bias estimates, and the cost function evolve throughout the batch-filter algorithm execution. This is discussed later in the context of algorithm functionality and robustness.

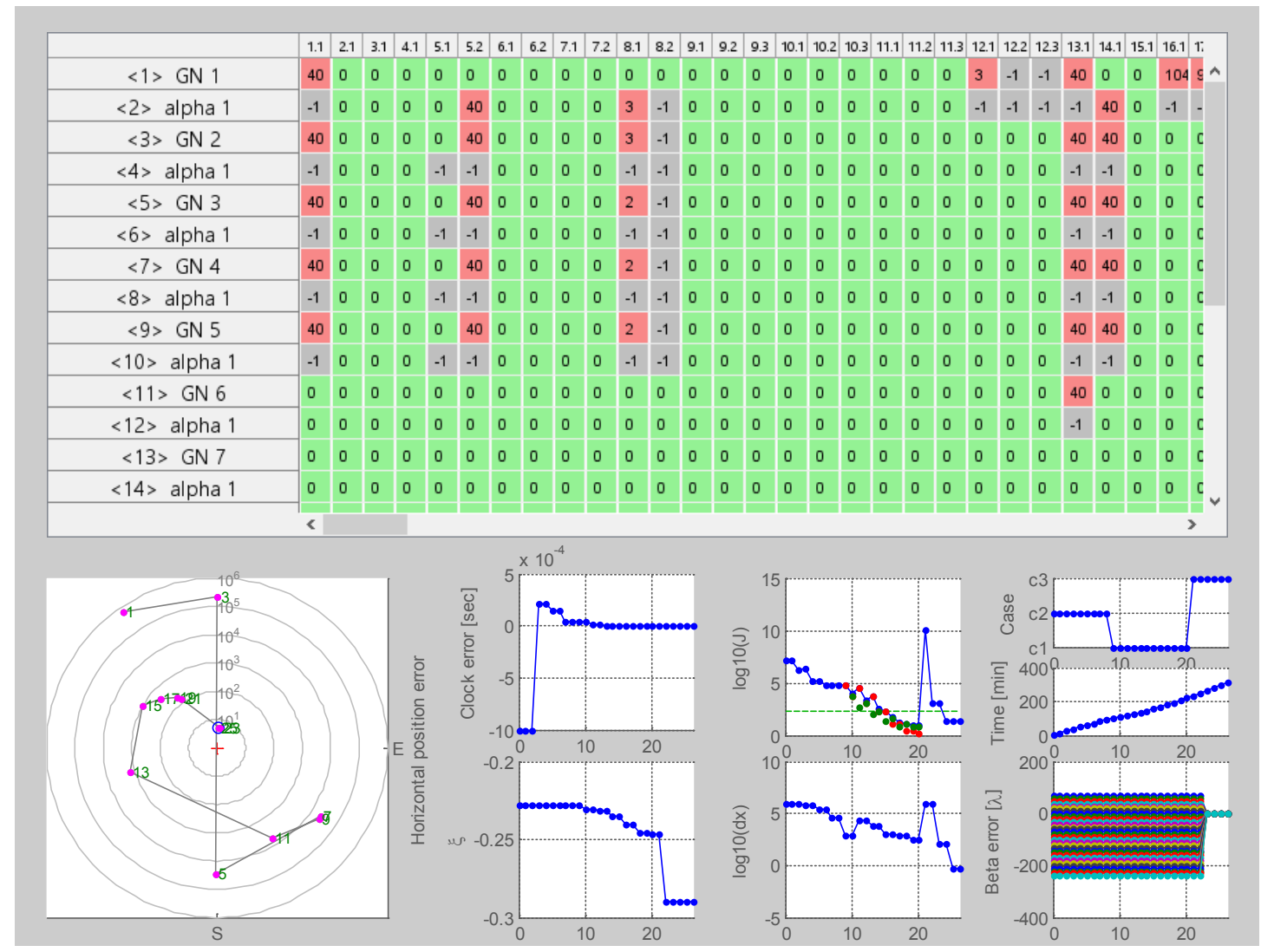

Figure 8: An example process monitoring screen for test case E0 in Nominal Scenario mode. 


\subsubsection{Execution modes}

In Nominal Mode, a single simulation execution is performed. No random measurement noise is added to the simulated pseudorange or beat carrier phase measurements. Errors are potentially enabled, however, for the a-priori ionosphere model. This means that the user can choose to use an erroneous ionosphere model for the a-priori ionosphere model that is input to the main solver algorithm. That is, the a-priori ionosphere model parameters that are input to the batch filter differ from the truth-model parameters that have been used to generate the pseudorange and range-equivalent beat carrier phase measurements using raytracing calculations.

This execution mode has proven essential for algorithm validation. It is also used with preliminary feasibility tests and algorithm assessments that evaluate the batch filter's behavior, particularly in the context of robustness. Another important use of this mode is the proximate prediction for the batch filter algorithm's performance in the presence of measurement errors that has been described in Section 4.3.

Performance Analysis Mode is usually used with Monte Carlo analysis. In this type of study, multiple sub-case executions that originate from a single nominal scenario are conducted. These sub-case executions are regarded as runs. Each batch filter run is executed with a unique set of pseudorange measurements that are generated by adding measurement noise errors to the pseudoranges and beat carrier phases from the corresponding nominal scenario. The nature of the applied errors is discussed later in the context of performance analysis. Ionosphere model errors are typically kept constant throughout a single Monte Carlo test case execution for reasons that are discussed in the 
performance analysis section. An exception is the process of full covariance analysis and validation that is presented in Section 6.5.1, for which random a-priori errors are generated such that each sub-case is given a unique set of a-priori ionosphere parameter errors.

\subsubsection{Data generation}

Computation for an $N_{e}(\underline{r})$ truth model utilizes a Chapman profile that is fit to an IRI model for a particular time. The model used in the simulation utilizes the release 2012 IRI Fortran code available on the official NASA website [36]. Settings for the binary options variable $j f$ are 'true' values for all of its entries except for entries 2-6, 21, 23, 2830 and 33-35. Among other things, these settings choose the URSI model rather than the CCIR model for the quantity $f o F 2$, and they turn off the auroral boundary and the auroral E-storm model. Additional details about these settings can be found at the NASA website that provides the IRI model.

A Chapman vertical electron density profile is fit to the IRI data, and this Chapman profile is the assumed truth profile at a given latitude and longitude. The fit is performed with a nonlinear least squares technique that minimizes the sum of (a) the squared difference between known and modeled electron densities at specific heights, and (b) the squared difference between known and modeled integrated electron density, which is also known as the Vertical Total Electron Content (VTEC). These two different contributions are weighted. This fitting procedure is carried out at each bi-quintic spline grid point to compute the $\log \left(h_{\max }\right), \log \left(h_{\mathrm{sf}}\right)$, and $\log (V T E C)$ values at each of these points. Afterwards, the needed 8 partial derivatives of each of these quantities are computed at 
the grid points by applying smoothness criteria to the resultant maps of $\log \left(h_{\max }\right), \log \left(h_{\mathrm{sf}}\right)$, and $\log (V T E C)$. The details of the fitting procedure and the spline derivative calculations are given in Section IV of Ref. [26].

This manner of generating a truth electron density model is thought to be reasonably representative of a possible real spatial electron density distribution. The most significant limitation of this approach arises with cases where the considered electron density distribution is characterized by abrupt changes in the height-dependent electron density profile. In such cases, the Chapman-based model fitting process is expected to exhibit significant residuals, indicating a subpar fitting.

The truth-model simulation and all of this dissertation's models take no account of HF radio wave absorption in the daytime D layer of the ionosphere. During the day, D-layer absorption could render some of the modeled ray paths unusable, as discussed in Ref. [37]. This does not occur at night, when the D-layer's electron density diminishes to a negligible value. The impact of D-layer absorption is left for a future study.

The simulation uses truth values of the $\underline{x}$ and $\underline{p}$ vectors in the vector pseudorange measurement model of Eq. (65) and in the vector beat carrier phase measurement model of Eq. (70). These measurement values are input directly into the main batch filtering algorithm with or without errors added, as mention above.

The simulation also generates an a priori estimate of the ionosphere parameter vector for use in the cost function of Eq. (79). This a priori $\overline{\underline{p}}$ vector differs from the truth $\underline{p}$ vector in significant ways. The method of generating appropriate differences, perhaps 
differences that are even a bit larger than one would expect in a real situation, is to use the IRI model with a significant time difference to generate $\overline{\underline{ }}$ via Chapman-profile fitting at a different date than the date used to generate the truth $\underline{p}$ by the same fitting technique. Such a choice ensures that the truth-model simulation is not using an unreasonably optimistic model of how well the filter's known $\overline{\underline{p}}$ would approximate the truth ionosphere.

\subsection{Normal Batch Filtering Algorithm Operation and Functionality}

A key question is whether, given a setup that has been examined and proven physically feasible, the algorithm is capable of causing its estimates of the unknowns to converge to the setup cost function's theoretical global minimum. In the early study of Ref. [5], it has been demonstrated that the estimation problem's significant nonlinearities do not pose a hurdle that prevents the solution from converging. Hundreds of test cases that have been studied in the scope of the current study have shown that, when a setup is physically feasible, the batch filtering algorithm will be able to reach solution convergence in the vast majority of cases.

\subsubsection{Ray-path solving attempts}

The iterative top-level Gauss Newton process will typically exhibit the characteristics

of the process that is documented in Fig. 8. This figure shows the process monitoring screen for a Nominal Scenario Mode execution of Test Case E0. Complete descriptions 
of the setup for this test case and other cases that are discussed in this subsection are provided in the results presentation of Chapter 7.

Computations for the different ray-paths, monitored in the top window of Fig. 8, might occasionally fail during batch estimation due to causes that will be discussed later. Yet, as the process progresses, the number of failing ray-path computations will decrease, so that for Gauss-Newton steps which are near the final step, at which convergence is achieved, the vast majority of ray-path computations are expected to succeed. Position error (plotted in the polar, logarithmic-scaled bottom left panel), may start as large as thousands of kilometers. The error decreases with each iteration of the Gauss-Newton process, until it eventually reaches its final value. Similarly, clock errors will generally converge to values that are very close to zero.

At the Gauss-Newton iteration level, proper algorithm functionality is first and foremost the ability to successfully compute trajectories for a sufficient number of raypaths with the same fundamental attributes of the true ray-paths. Fulfillment of this requirement may be challenging, especially when the initial guess for the various unknowns is poor. Figure 9 shows four of Test Case 3411's twelve ray-paths. The other eight ray-paths are not shown for the sake of clarity. Ray-path 1 is shown in blue, raypath 6 is shown in green, ray-path 9 is shown in red, and ray-path 11 is shown in cyan. The thick lines indicate true ray-paths, all of which end at the true location of the receiver, i.e., at the center of the black square. The thin lines show the trajectories of these four ray-paths as computed by the ray-path solver during an early Gauss-Newton iteration. These curves end at the erroneous location of the receiver that is marked with a 
black X. By design, the computed ray-paths, albeit different from the true ones, have the same basic characteristics as the true ray-paths in terms of number of ray-hops and the direction of arrival at the receiver at a qualitative level, i.e., whether signals arrive from below or above the horizon. A closer look at the last segment of all ray-paths confirms that for the blue and green ray-paths, the signal arrives at the receiver from above for both true and computed trajectories, while for the red and cyan ray-paths it arrives from below. Thus, for this iteration of this batch filter case and for these four ray paths, the batch-filter functions as expected and needed.

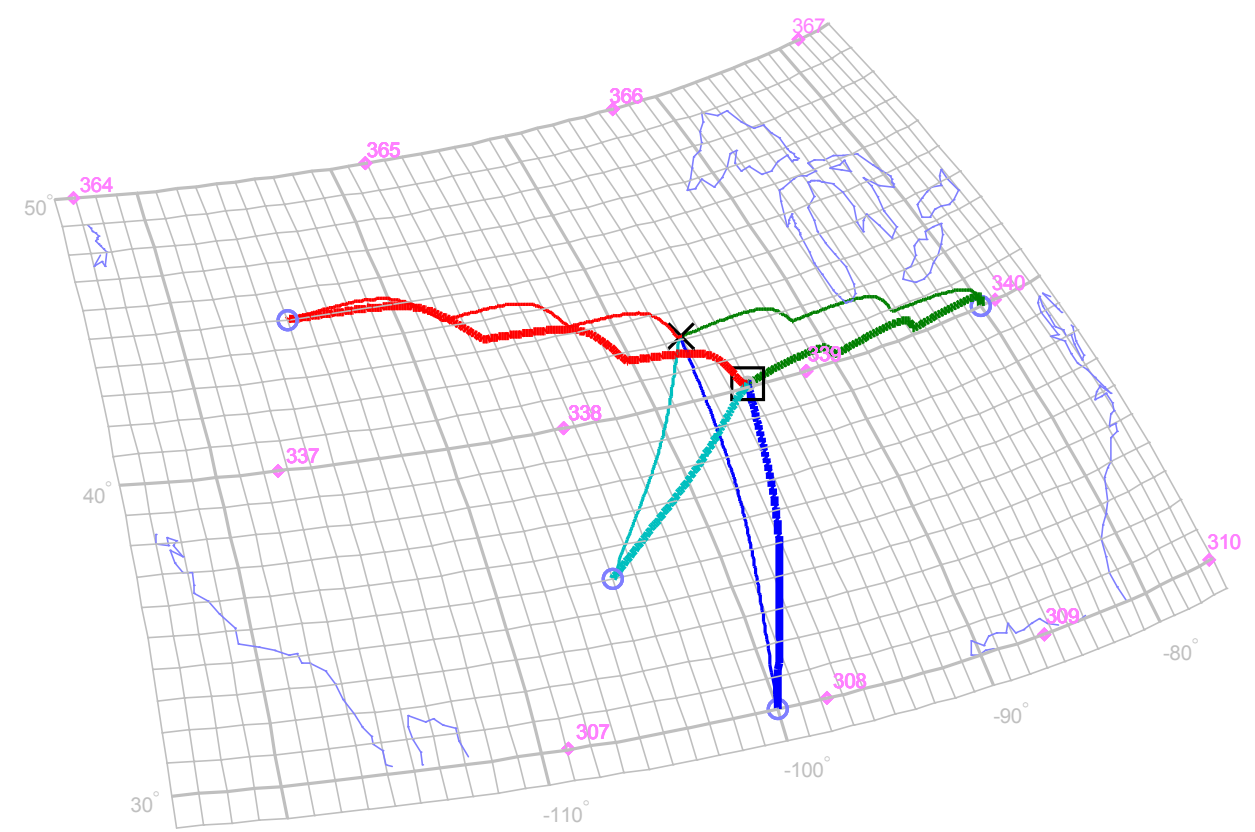

Figure 9: Proper algorithm functionality is evident in successfully computed ray-paths (not all presented) that have the same characteristics as their true ray-path equivalents. This figure plots true (thick lines) versus estimated (thin lines) ray-paths for an early iteration of the Gauss-Newton process for Test Case 3411.

The process monitor screen for Test Case 3811 is shown in Fig. 10. For this test case, the location of the ground stations, some of which are very far from the receiver, resulted in several failures while attempting to solve for the ray-paths. These failures, occurring 
mainly in the early iterations of the batch filter calculations, usually do not constitute a significant challenge to the algorithm's ability to ultimately bring the solution to convergence. This means that later Gauss-Newton iterations, which are not shown in the upper plot of Fig. 10, have an increasing number of green cells, until ultimately all cells are green for the last Gauss-Newton iteration.

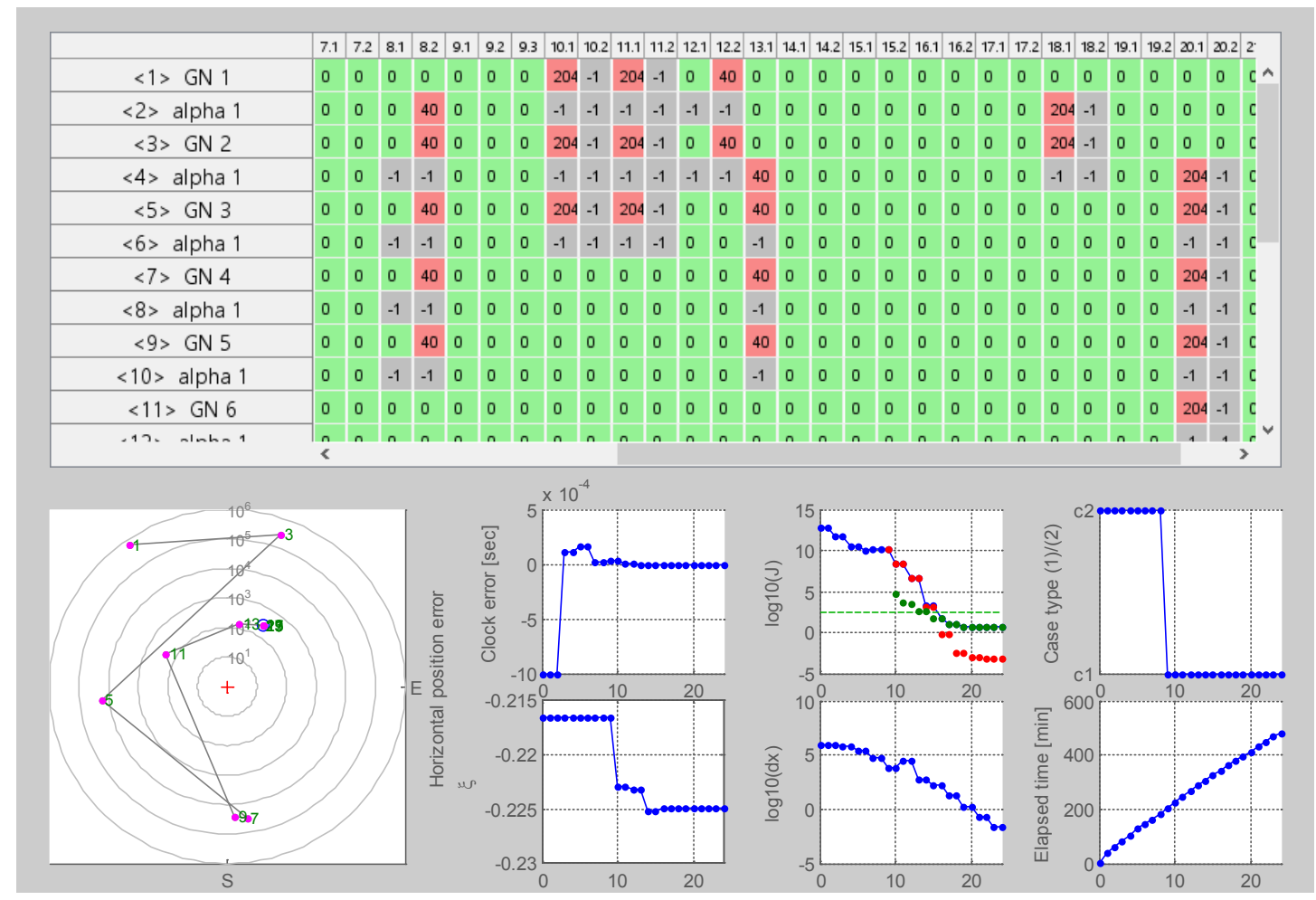

Figure 10: Process monitoring screen for Test Case 3811 in Nominal Scenario mode, demonstrating occasional failures in attempts to solve for ray-hops and ray-paths during execution of the batch filter.

Even with many more failures in early ray-path solving attempts, as in Test Case 3531

(Fig. 11), the unknown parameters' estimates were successfully driven to their optimal values. With an initial IEI value of -0.0660 , this test case is characterized by significantly larger errors in the a priori ionospheric model than for the previous example. The upper 
panel of this figure shows ray-hop calculation results for ray-paths 1-12 and process steps 8-22. It is evident that, for these early process steps, many ray-hop computations, and consequently, ray-path computations, have failed.

At step 20 the algorithm changed its mode to Case 1, meaning that, starting at this process step, corrections to the ionosphere model were enabled as part of the solution procedure. The immediate impact of mode switching is a major degradation in the quality of estimates for the ionosphere model, evident in extreme $\xi$ values that are not typical.

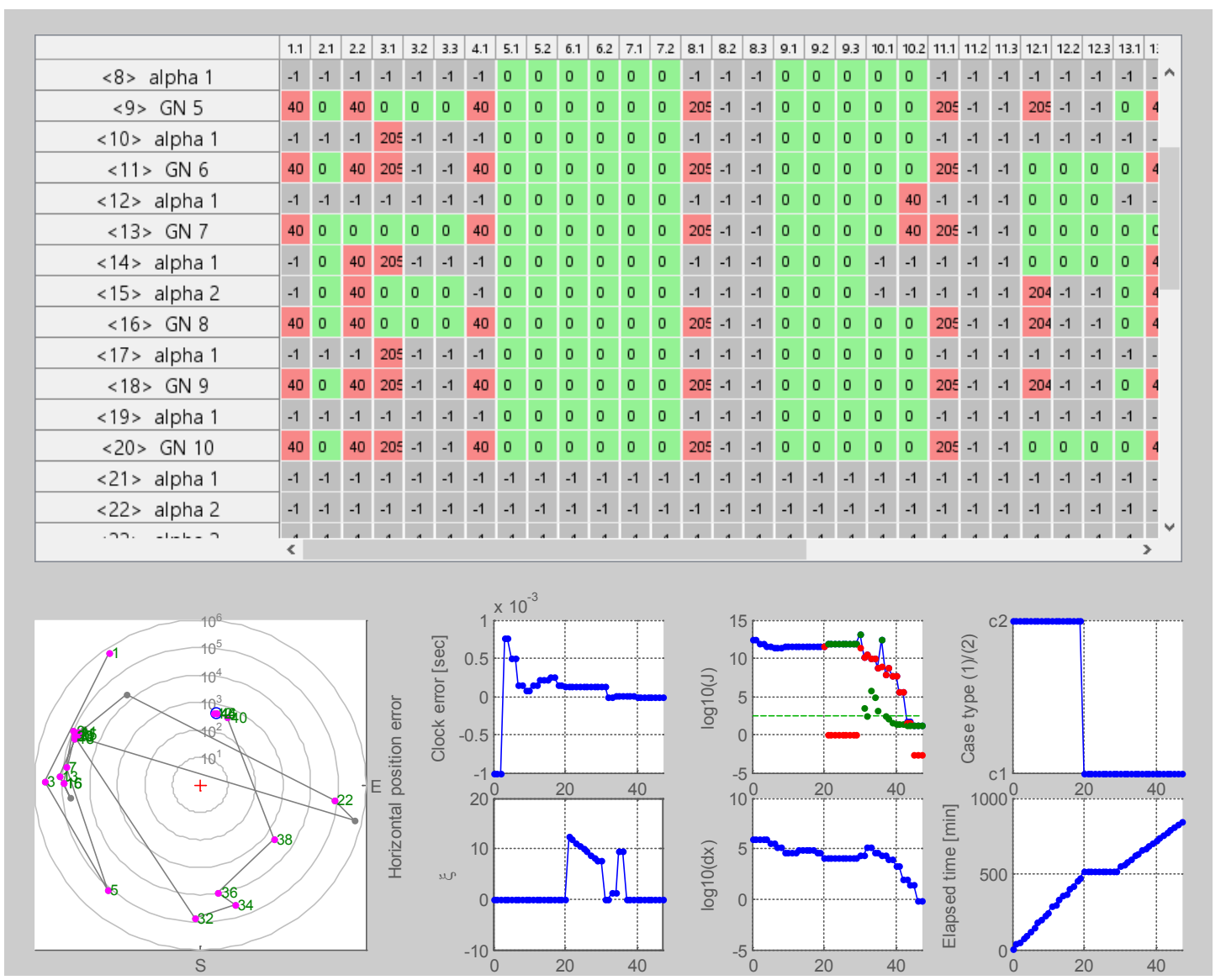

Figure 11: Process monitoring screen for Test Case 3531 in Nominal Scenario mode, demonstrating massive ray-paths solver failures at the early stages of the iterative batch filtering process due to a very poor a priori ionosphere model. Both final position error and final IEI $\xi$ are small. 
This is most likely the cause for the excessive amount of failures in the attempts to solve for ray-paths during process steps 21-29, where the algorithm was seeking a proper scaling factor, $\alpha$, that would yield a decrease in its cost function. Starting at step 30, however, where a decrease in the cost function was achieved for a small value of $\alpha$, the solver was able to solve an increasing number of ray-paths, so that all ray-path and rayhop computations succeeded at process steps 38 through 47 (not shown). As with the vast majority of cases, the $\xi$ value at the end of the process, -0.965 , is lower than its initial value.

\subsubsection{Cost function evolution}

Cost function values are displayed on a logarithmic scale in the top panel of the second-from-right column of the bottom two rows of each monitoring screen. The blue line marks the total cost while the dots mark the values for the measurement residuals cost terms and the a priori ionosphere cost terms of Eq. (79). The measurement residuals terms and the a priori ionosphere terms are denoted in this plot by red and green, respectively. Considering Fig. 11 again, it can be observed that the general trend for the cost curve is a decrease, but for some Gauss-Newton iterations the total cost appears bigger than for their preceding iterations. In many cases, this is due to the strategy of using variable subsets of measurements that has been discussed earlier in subsection 4.2.2. Thus, in spite of appearance, a decrease in the cost function does occur for an appropriate subset of measurements. Cost function curves may additionally include sequences of process steps with what might appear to be a sudden increase in the cost function followed by a monotonic decrease. This is caused by the sub-process where the 
algorithm seeks the step scaling factor that yields a reduction in the cost function. These intermediate values should not be confused with the major Gauss-Newton iterations' final values, for which monotonic decrease is guaranteed. As mentioned before, the latter are designated $<n n>G N$ in the upper panels of the monitor screen, where $n n$ is the process step number. Finally, with the combined code/carrier ranging algorithm, there will always be a single significant increase in the cost function when carrier-phase measurements are eventually incorporated into the filter (e.g., at step 21 in Fig. 8).

An additional trend in the cost function plot is the constant decrease in the magnitude of the ionosphere-related term (green dots). This is a counter-intuitive result. One would expect this cost to start at zero when the estimated $\underline{p}$ equals its a priori value. It should increase from there as it finds a better ionosphere parameter to fit the measurements by estimating a non-zero deviation from the a priori value that is also its initial guess. This counter-intuitive result can be attributed in part to the variable scaling method that is used with this term.

Finally, the decreasing measurement-related cost term eventually converges to very small values, thus indicating that the algorithm has succeeded in bringing the differences between measured and computed range-equivalent group delays and beat carrier phases very close to zero.

\subsubsection{IEI evolution}

Recall that the Ionosphere Errors Index, $\xi$, can be regarded as an error metric for the corresponding estimate for the ionospheric model. The final value for the IEI normally exhibits a decrease from its initial value (except in rare cases). Nevertheless, a significant 
temporary increase in its value, as in Figs. 11 and 12, is common. In the latter example, the initial IEI value is -0.1440 . At step 8 , which is the first Gauss-Newton iteration where the algorithm utilizes the full cost function formulation of Section 4.1, this value increases to 0.1507 . In the following steps, the IEI exhibits constant decreasing towards its final value of -0.1499 .

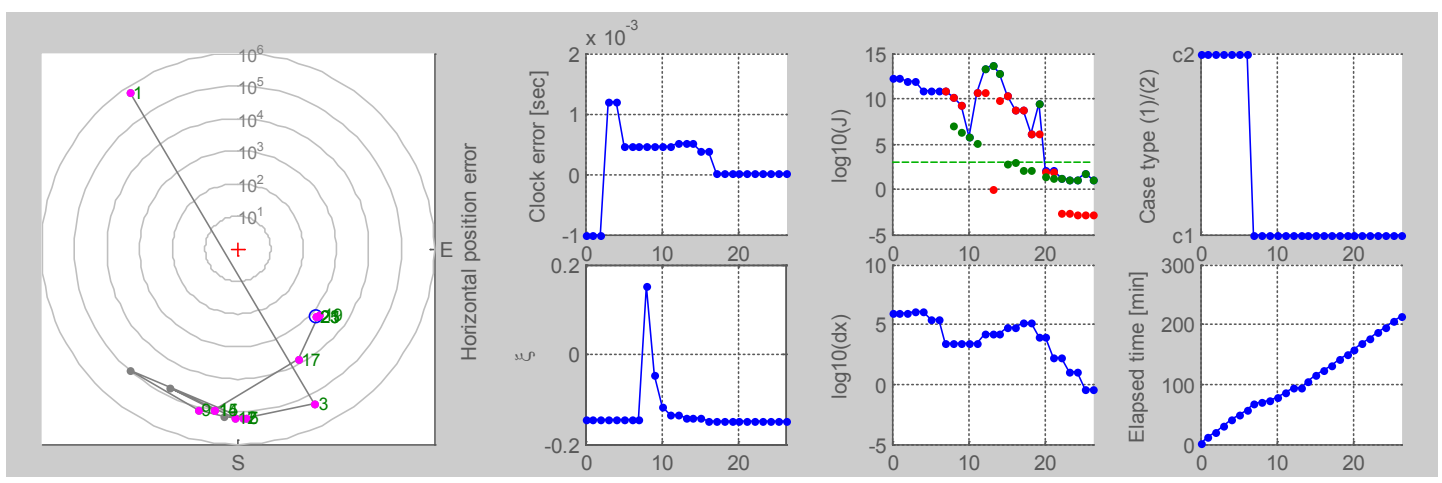

Figure 12: Process monitoring screen (partial view) for Test Case 3431 in Nominal Scenario mode. Note the behavior of the IEI parameter $\xi$.

\subsection{Process failure patterns and solution divergence}

\subsubsection{Failure types}

Most test cases exhibit proper batch filtering algorithm functionality that results in valid a posteriori position, timing, and ionospheric model estimates. It has been observed, however, that for both Nominal Scenario and Performance Analysis execution modes, occasional process failures may occur. In such cases, the filtering algorithm will generally fail to converge. This failure is indicated by the norm of the vector $\Delta \underline{x}_{\mathrm{c}}$ of Eq. 
(86), designated $d x$, that is used to detect convergence: it does not decrease below a predefined threshold. Figure 13 shows the process monitoring screen for run 0002 of a limited Monte Carlo analysis that was performed with Test Case 3411A. Clearly, at none of the 104 steps performed prior to filter algorithm termination was the combined position/time/ionosphere model estimate close to its optimal value and hence, to convergence. This is evident in the position error pattern, as well as in the values that the cost function takes throughout the iterative process.

Convergence failure is also evidenced by the $\log 10(d x)$ plot in Fig. 13, which is the second-from-right bottom panel of the figure. The value of $\log 10(d x)$ never falls below 2 , and it oscillates up and down during the entire process. In the preceding examples of process monitoring screens in Figs. 10, 11, and 12, this metric starts near 5, it exhibits a general downward trend, and it ends near 0 .

A second type of process failure is demonstrated with the Nominal Scenario mode execution of Test Case 3522, shown in Fig. 14. Unlike with the previous example, where the IEI appears steady at a value of about -0.2 (the reader should ignore the peaks that are artifacts caused by the step-size halving sub-process), in this test case the IEI reaches very large values of more than 8 . This indicates a divergence for the Chapman parameter estimates. Position errors are consistently very large. 


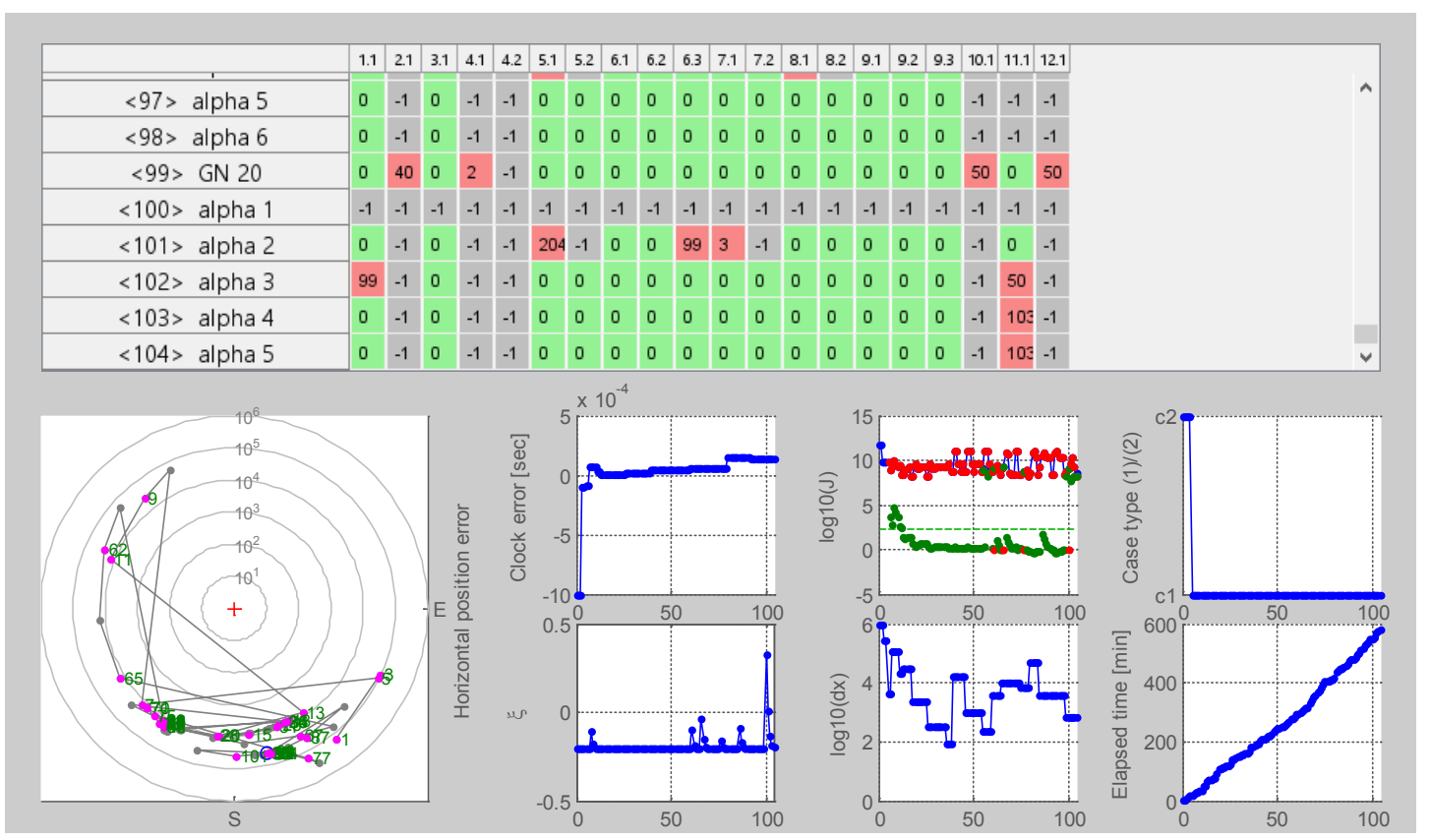

Figure 13: Process monitoring screen for run 0002 of a Monte Carlo Analysis conducted for Test Case $3411 \mathrm{~A}$. With more than 100 process steps, the algorithm failed to drive the unknown position solution closer to its optimal value, yet $\xi$ values remain fairly steady.

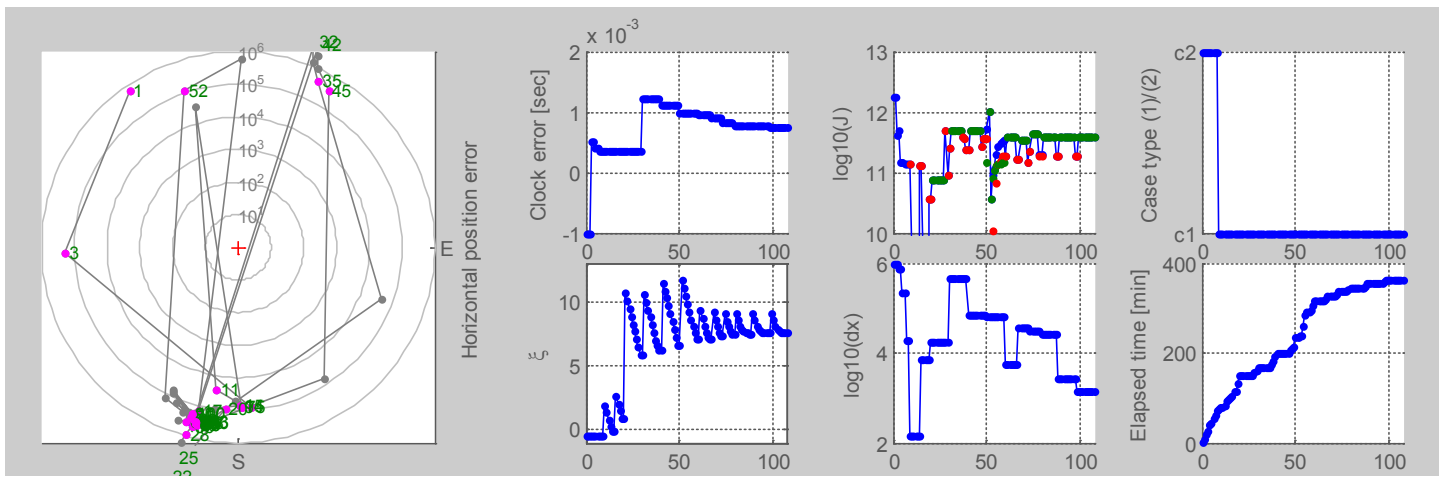

Figure 14: A partial view of the process monitoring screen for a Nominal Scenario simulation of Test Case 3522. Magnitude of errors for all unknowns indicates process divergence.

With a third type of algorithm failure, estimates for position, clock error and ionospheric model are close to their optimal values, yet the algorithm fails to drive those values to convergence. Figure 15 shows the process monitoring screen for run 0087 of a 
Monte Carlo analysis for Test Case 3911. For process steps 20 and later, position error remains bounded within a limit of a 100 meter radius circle that is denoted $10^{2}$ in the polar position error plot. At the same time, the IEI exhibits stability near a value of -0.2463 , and the receiver clock error is steady at -581.3 microseconds. Yet, the algorithm fails to drive the unknowns to their optimal values when running in Nominal Scenario Mode. Recall that the optimal solution can be closely predicted using the linear approximation of Eq. (99) and the known receiver location and true ionosphere parameters.

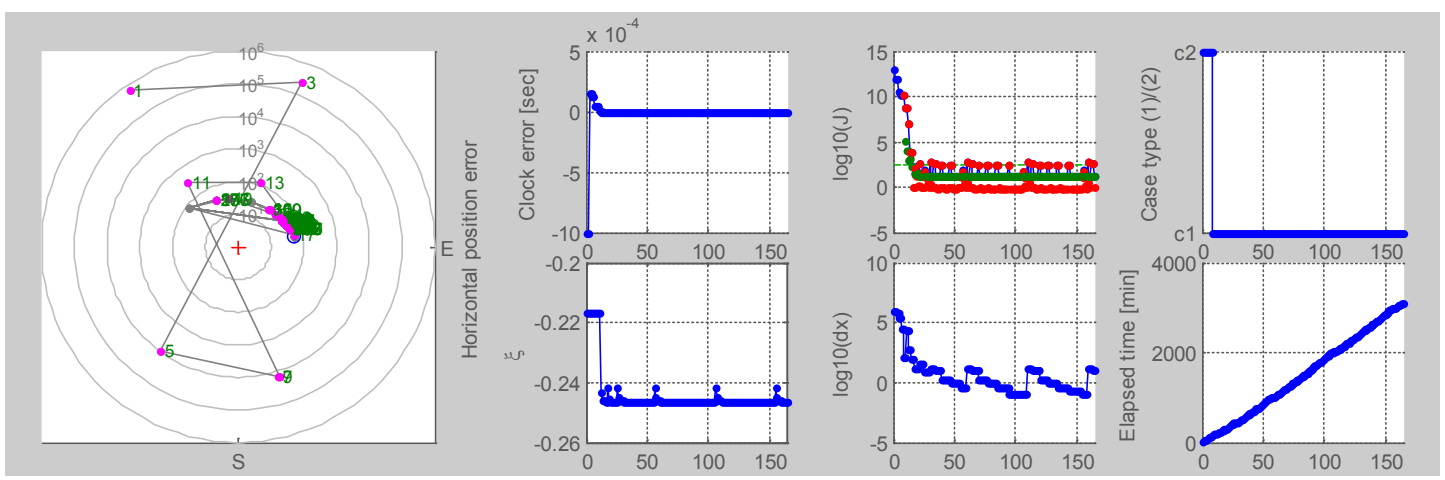

Figure 15: Process monitoring screen for run 0087 of a Monte Carlo Analysis conducted for Test Case 3911. Estimates remain close to their optimal values, yet the algorithm fails to bring them to convergence.

\subsubsection{Causes of algorithm failure to converge and known algorithm limitations}

A study of several test cases, including the above run 0087 for Test Case 3911, revealed a weakness in the optimization process. When the ray-path solver makes an attempt to solve for the bounce points, as in Section 4.2.6, with a signal ray-path that eventually arrives at the receiver from below, a second, valid solution that satisfies Eq. (29) may be obtained instead of the desired solution. Figure 16 illustrated the nature of 
this ambiguity. The top panel plots the trajectories for the true (blue) and estimated (green) ray-path 33. Both trajectories start at the known position of a ground station that is denoted with a blue circle and end at two points that are roughly 100 meters apart. The closer-view bottom panel plot shows parts of the two trajectories in the vicinity of the true and estimated receiver locations. It can be observed that the last segment of the estimated ray-path is in a direction that is nearly opposite to the direction of the previous hop as it hits the ground.

Further study has shown that the cost function that is used when solving for the bounce points in this case converges to a local minimum that is non-zero. This physically infeasible solution is most likely due to a poor choice of an initial guess for this raypath's bounce points. Worse, it results in computed range equivalent group delay and carrier phase measurements that are significantly different from the true ones. It has been examined and shown that this discrepancy prevented the process from converging to the optimal estimates in this case.

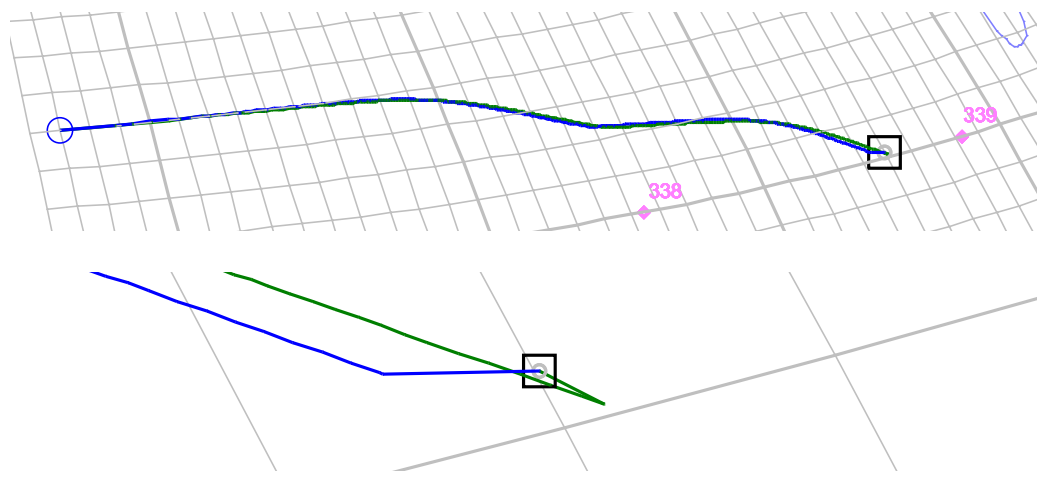

Figure 16: True (blue) and computed (green) trajectories for ray-path 33 of run 0087 of a Monte Carlo analysis for Test Case 3911. Top: the full ray-paths; Bottom: a closer look near the true (blue) and estimated (green) trajectory end points. 
A second, more commonly encountered cause for ranging discrepancies arises from the ambiguity of the direction at which a signal will eventually approach the receiver. This is demonstrated in Fig. 17 which shows the true (blue) and estimated (green) raypath trajectories for ray-path 9 of Monte Carlo run 0002 of Test Case 3411A. Both trajectories originate at the same known location of the transmitter that is denoted by a blue circle. While the true signal arrived at the true location of the receiver from above, the computed direction of arrival at the (erroneous) location of the receiver is from below. As both trajectories have the same number of hops and bounce off points on the Earth's surface (labeled in the plot), they constitute two legitimate solutions for very similar setups that only differ in the slightly different locations for the receiver. The difference is that, after the last bounce, one path travels directly up to the receiver (the estimated path), while the other path, the true blue path, refracts off of the ionosphere and comes back down to the receiver.

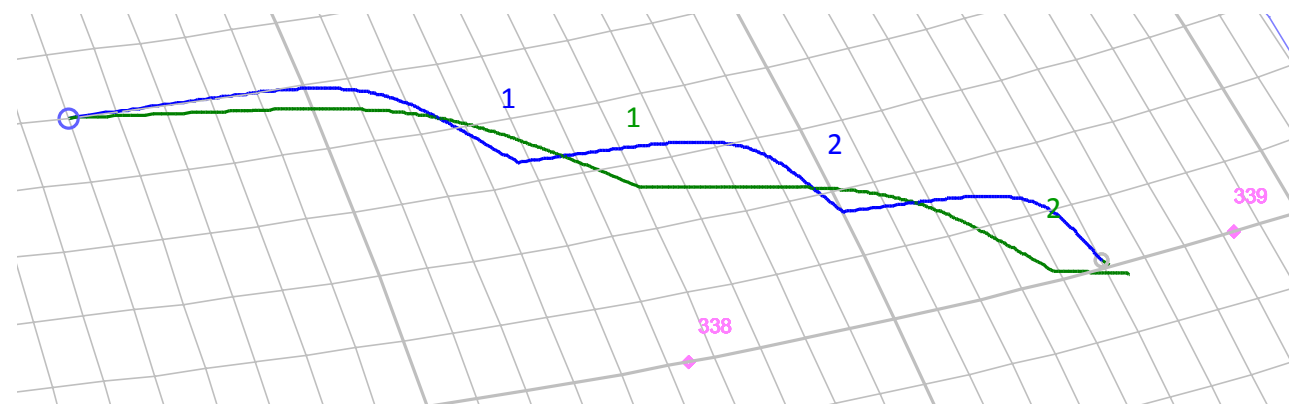

Figure 17: The true trajectory for ray-path 9 (blue) and the estimated trajectory (green) for that ray path, calculated for Monte Carlo run 0002 of Test Case 3411A. Inconsistency in signal direction of arrival yields significant, unaccounted for measurement errors. 


\subsubsection{Used and suggested solutions}

The measurements rejection mechanism that has been described in Subsection 4.2.3 has been proven somewhat effective in addressing the issues that have been presented in the previous subsection. In most cases, selective measurements rejection resulted in elimination of undesired algorithm behaviors such as those described in Subsection 6.3.1.

An additional means for overcoming ranging discrepancies would be rejecting measurements for which measured and computed trajectories consider different number of ionospheric reflections, and consequently, result in one trajectory approaching the receiver from below, while the second approaches from above. With a real system, the actual direction of arrival might be roughly determined by considering the signal power and the antenna gain pattern.

\subsection{Solution Convergence to the Problem's Optimal Solution}

A key event in the execution of the main solver algorithm is identifying solution convergence. As shown earlier, the step magnitude criterion, $d x$, is utilized in determining whether convergence has been reached, so that the batch filtering algorithm measures the magnitude of a vector whose first three entries are the three components of the ECEF representation of the corrections to the estimated receiver position, and the fourth entry is the product of the speed of light and the change in the estimated receiver clock offset. 


\subsubsection{Cost function characterization through 3D mapping}

An inherited drawback in using the Gauss-Newton algorithm is the potential hazard of converging to a local cost function minimum that is not the global minimum, but to believe that it is the global minimum. In hundreds of test cases and tens of thousands of Monte-Carlo runs studied, none of the iterative processes resulted in convergence to minima different than the global minimum which can be approximately determined based on Eq. (97), as mentioned earlier. In a further effort to assess the likelihood of such occurrence, the following analysis has been performed.

A test case has been considered with a receiver located at latitude/longitude/altitude (LLA) $\left[40.1^{0},-95.1^{0}, 10000 \mathrm{~m}\right]$ that has been assumed to receive signals along 12 ray-paths transmitted from different ground stations. The position solution that has been obtained by solving the standard minimization problem is located at coordinates $[-72 \mathrm{~m},-925 \mathrm{~m},-$ $212 \mathrm{~m}$ ] in a Vertical-East-North (VEN) local-level reference frame whose origin is located at the receiver's true location. This optimal solution is marked by the magenta X in Fig. 18. A three dimensional grid has been defined in a volume region of $500 \times 3000 \times 3000 \mathrm{~m}^{3}$ in which the optimal solution for position lies. Each point of this grid has been used to define a unique related optimization problem for which position is known and fixed, while corrections are allowed to clock error and to the ionosphere model, as in Subsection 4.1.2 and Eq. (81). Each of these 1859 optimization problems has been solved, and all resulting final cost functions have been computed. The result, in the form of a 3-dimensional spatial cost function map, is presented in Fig. 18. Plotted values are base-10 logarithms of the computed cost function values. 
The most significant pieces of information in this plot are that (a) the minimum is unique in the limited region studied, and (b) in the vicinity of the optimal solution, the cost function has its closest spacing of contours of constant cost nearly in the vertical direction. The first result partially addresses the non-uniqueness concern discussed above, although it is clear that a bigger region should be studied in order to strengthen this result.

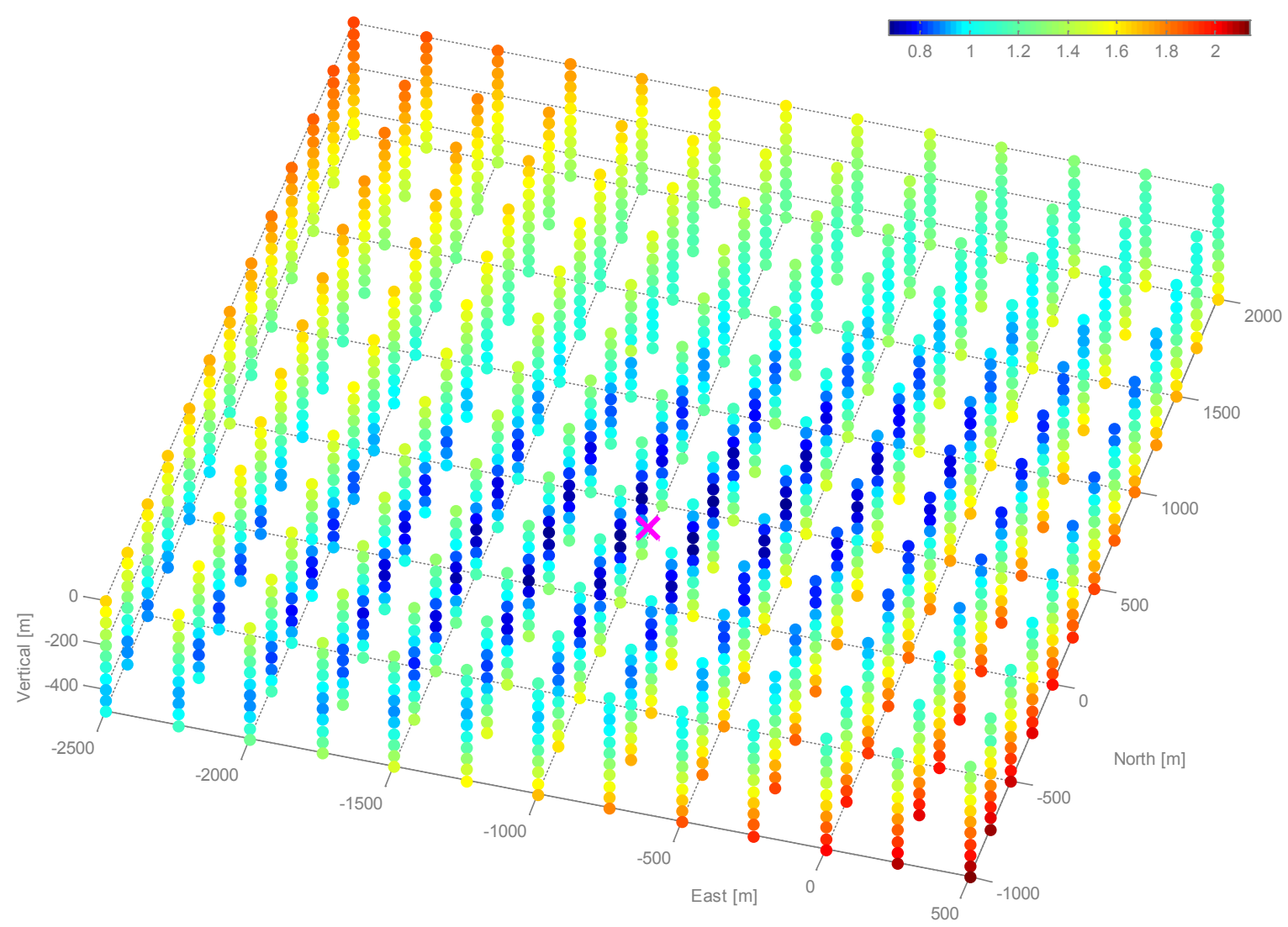

Figure 18: A spatial cost function map for a 12-ray-paths test case. Base-10 logarithm of cost function values, shown by color coding, have been computed in the vicinity of the optimal solution for the receiver location.

An attempt to perform a similar analysis for a much larger region was unsuccessful mostly due to a high rate of failed solving attempts for tested grid points that are far from 
$\underline{r}_{R}$ 's optimal solution. The second result indicates that vertical position accuracy will be the best, which is consistent with results that will be presented in the performance analysis chapter.

\subsubsection{Convergence validation}

As a measure of validation for the convergence criterion, numerical computations have been examined and compared to their expected, theoretical values. Writing the firstorder optimality necessary condition for the cost function of Eq. (79) yields

$$
\begin{aligned}
\underline{0} & =\left[\begin{array}{l}
\left(\partial J_{1} / \partial \underline{x}\right)^{T} \\
\left(\partial J_{1} / \partial \underline{p}\right)^{T}
\end{array}\right] \\
& \approx-\left[\begin{array}{l}
H_{x}{ }^{T} \\
H_{p}{ }^{T}
\end{array}\right] R^{-1}\left[v_{z}-H_{x}\left(\underline{x}-\underline{x}_{\text {rue }}\right)-H_{p}\left(\underline{p}-\underline{p}_{\text {true }}\right)\right]+\left[\begin{array}{c}
\underline{0} \\
M^{-1}\left[\underline{p}-\underline{p}_{\text {true }}-\underline{\varepsilon}_{p}\right]
\end{array}\right]
\end{aligned}
$$

One should expect the right-most term to be non-zero in the general case, as the term $\underline{\underline{p}} \underline{\underline{p}}_{t r u e^{-} \underline{\underline{p}} p}$ is zero only in the improbable case where the estimated ionosphere parameters equal the a-priori ionosphere parameters. This also means that the left-most term of the right-hand-side of the equation equals the negative of that term once minimization has been achieved.

This convergence condition has been studied with the same test case setup used in the above cost function mapping analysis. It has been observed that, while the two vector terms of the right-hand side of Eq. (124) consist of values whose magnitudes are in the order of $10^{-5}$ to 1 , the sum of the two is a vector whose entries have orders of $10^{-10}$ to $10^{-5}$. 
Similar results were obtained with additional test cases. This result demonstrates that the Gauss-Newton iterations converge at least to a local minimum that satisfies the first-order optimality necessary condition.

\subsubsection{Rate of convergence}

A distinct advantage of the Gauss-Newton method over the plain Newton's method is in saving the need for computing the second derivative Hessian matrices of the measurement model components. In the scope of this study, these computations would be extremely costly in terms of computational effort and therefore undesired. A significant amount of computation time is expected to be saved as long as the solution's convergences rate does not become significantly inferior to that of an equivalent higherorder nonlinear least-squares method.

Reference [32] shows that the Gauss-Newton method will generally exhibit a convergence rate that is guaranteed to be similar to that of Newton's method as long as the first-order term in the explicit formulation of the Hessian, which is given in Eq. (10.5) of that book, dominates the second-order term. With several test cases studied, it has been examined and shown that as long as the current guesses for the unknowns $\underline{x}$ and $\underline{p}$ are in a small region about their optimal values, the convergence is indeed fast. The latter statement is based on a number-of-unchanged-digits criterion, meaning that for the estimates of the receiver location coordinates, the number of significant digits that remain unchanged at a given Gauss-Newton iteration compared to the previous iteration almost doubles with each iteration. This means that for most of the iterative process, that 
typically takes place with the unknowns close to their optimal values, the convergence rate is presumably only slightly lower than that of a potential higher-order solver, and therefore, given the saving in computational time per process iteration, it is reasonable to assume that the total process time is less than a full Newton's method algorithm, making the Gauss-Newton method a reasonable choice in this case.

\subsection{Covariance Analysis Validation}

\subsubsection{The case of random ionosphere parameters errors}

The result given in Eq. (100) has been validated using the truth-model simulation with two test cases. Each test case of 200 randomly generated runs (sub-cases) constitutes a limited Monte Carlo analysis that considers variations to both group delay measurements and ionosphere parameters. Independent errors with a standard deviation of 1 meter were considered for ranging measurements. Random errors for the a priori ionosphere parameters were generated using a matrix that is based on a scaled variant of the covariance matrix $M$ that was described in Section 4.2.4, where the matrix has been scaled down by a factor of 10 in order to ensure that the majority of the resulting subcases are physically feasible and can be solved with the existing batch filtering algorithm. This results in typical small IEI values of about -1 for the different sub-cases. A scaling factor of 0.1 was additionally applied to $\zeta$ so that the generated errors are consistent with the matrix that is considered by the batch filtering algorithm. 
Figure 19 shows a layout of position errors that were obtained with a 200 runs MonteCarlo analysis and $90 \%$ error ellipses that have been generated based on the theoretically computed covariance matrix. With 17 points in the North-East errors plot falling outside the ellipse and 22 points falling outside the ellipse in the Up-East plot, it can be concluded that the computed $a$ posteriori error covariance and the Monte-Carlo-based analysis are consistent. It is also evident that the estimates are unbiased, as discussed earlier. The setup for this test case, marked 5511, includes 21 ray-paths that reach the receiver.
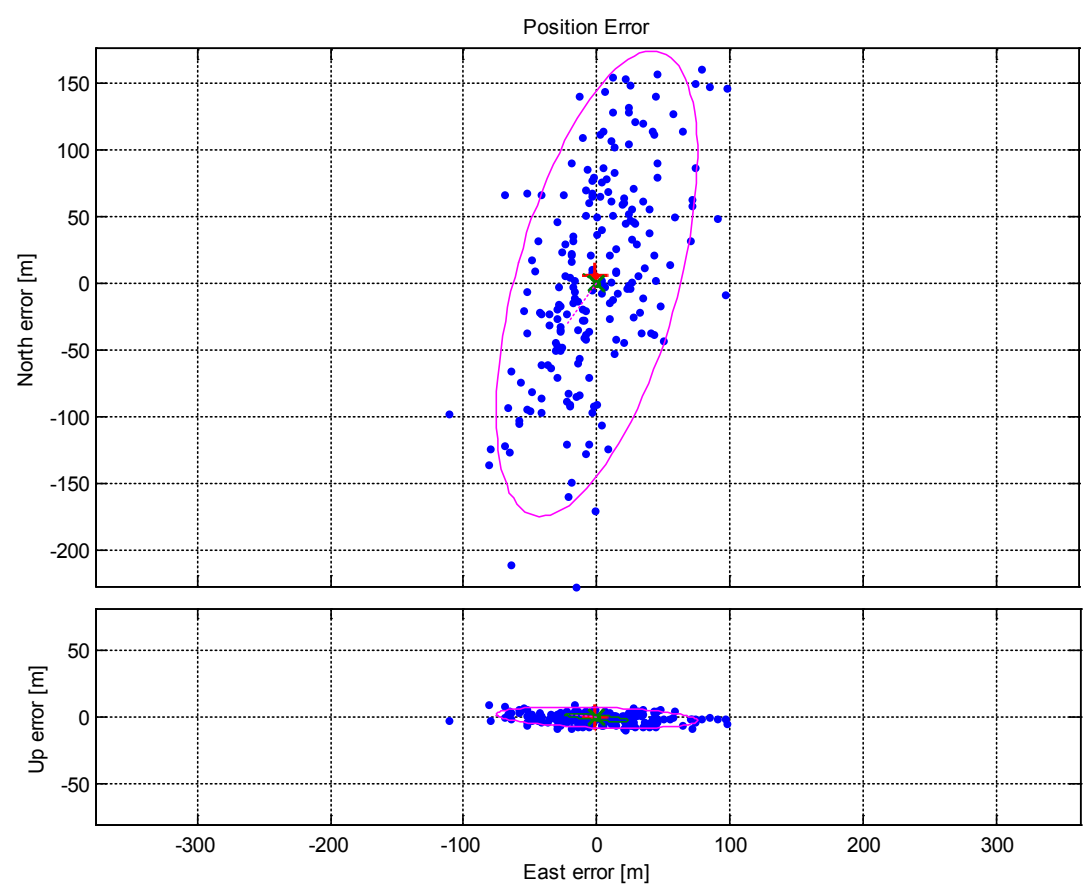

Figure 19: Monte Carlo and theoretical position error distributions for Test Case 5511. 17 out of 200 points fall outside the horizontal $90 \%$ error ellipse and 22 points fall outside the vertical $90 \%$ error ellipse.

Similar results have been obtained for test case 5611, which has a different groundstation setup and 33 ray-paths. Twenty two dots fall outside the horizontal $90 \%$ error 
ellipse, and 21 dots fall outside the vertical Up-East 90\% error ellipse. As with the first case, the results, shown in Fig. 20, demonstrate consistency between the analytically computed and Monte Carlo generated error distributions. This second analysis for the case of random ionosphere parameter errors completes the theoretical covariance analysis validation for the case of random errors in the ionospheric model.
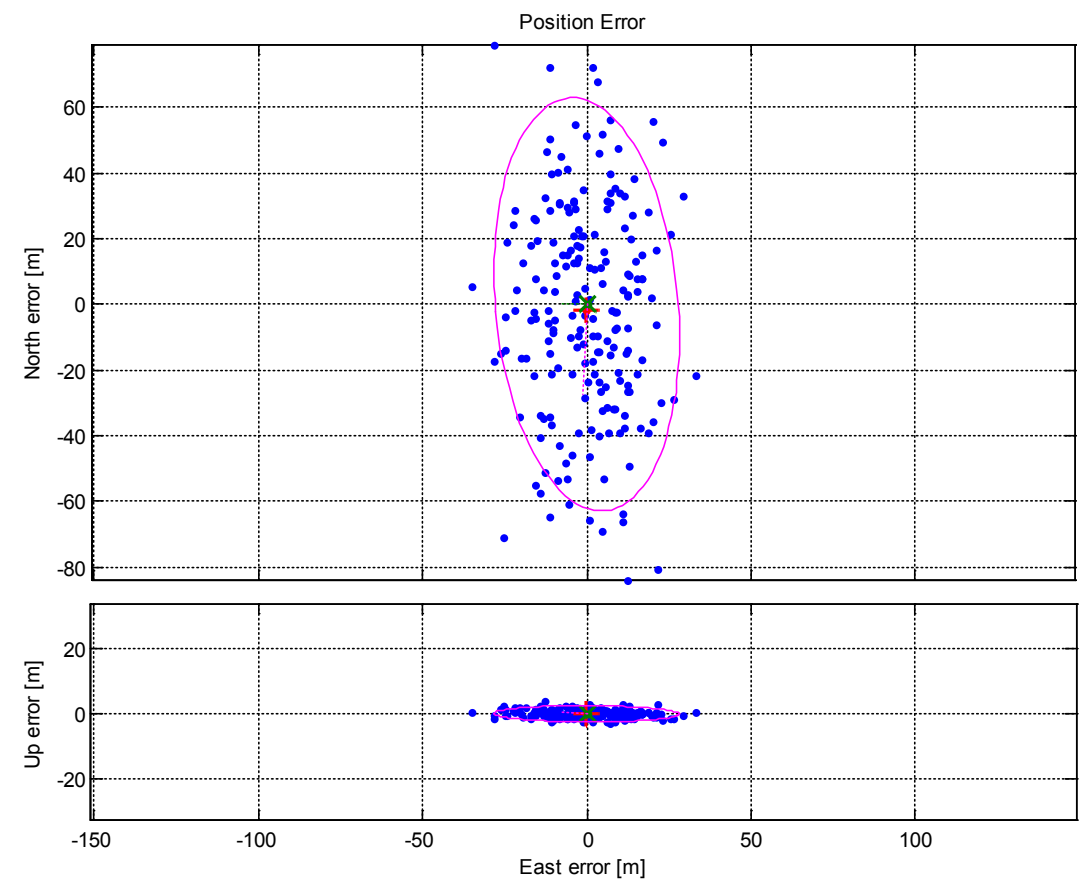

Figure 20: Monte Carlo and theoretical position error distributions for Test Case 5611. Twenty two dots out of 200 fall outside the horizontal $90 \%$ error ellipse, and 21 , dots fall outside the vertical Up-East $90 \%$ error ellipse. 


\subsubsection{The case of constant a priori ionosphere parameter errors}

In this subsection, a fixed, non-zero $\underline{\varepsilon}_{\mathrm{p}}$ vector is considered. This case of constant $a$ priori ionosphere parameters errors is of importance for system performance analysis of the next chapter. The goal is, therefore, to verify that the position error distribution is consistent with the theoretical covariance matrix that is given by Eq. (102), and with the bias term that equals the right most term of Eq. (99). The latter computed term is marked with a green $\mathrm{X}$ in the following figures, and the covariance matrix is used to compute theoretical $90 \%$ error ellipses that are plotted around the corresponding theoretical mean values. Its location can be compared with the actual mean of the Monte-Carlo position locations that is shown as a red + mark.

Nineteen of 200 points fall outside of the horizontal 90\% error ellipse shown in Fig. 21. This agreement between theoretical and practical error distribution patterns validates both the error bias and covariance analyses that are described in Section 4.4.2, as the plotted 90\% ellipses are centered (red cross) close to the predicted mean error (or bias) which is marked as a green X. The presented data are based on test case 3511 that is characterized by 21 received ray-paths and an a priori IEI of -0.2276 .

Agreement with Eqs. (99) and (102) is demonstrated by two additional test cases that are characterized by different setup properties. These test cases are used to further study the scope of validity for the results of these equations. Based on Eqs. (99) and (102), the test case with a high a priori IEI is expected to demonstrate a larger position error bias, while larger measurement errors are expected to result in a larger $90 \%$ error ellipse. 


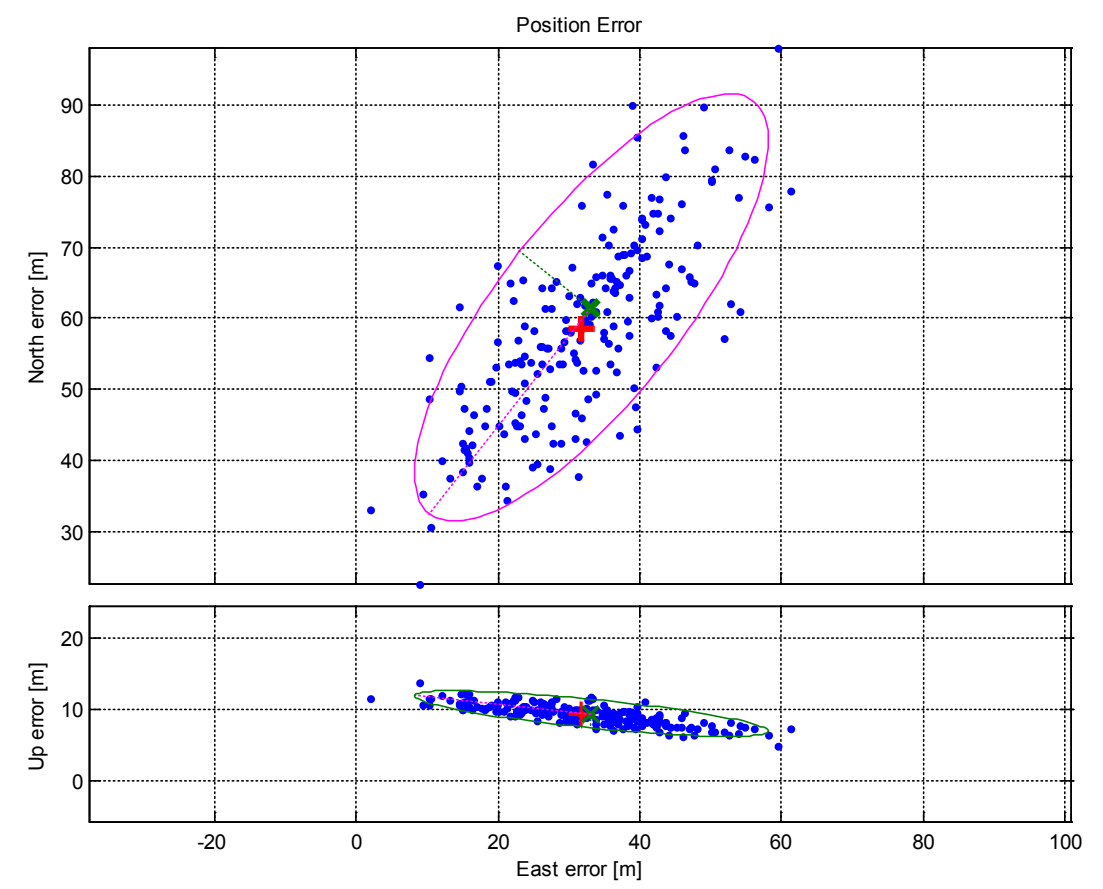

Figure 21: Empirical and theoretical position error distributions for Test Case 3511. This plot demonstrates mean error and error covariance consistency for the important case of constant a priori errors in the parametric ionosphere model.

Test Case 3531 (Fig. 22) has a high a priori IEI value of -0.0660 which lies within the $95^{\text {th }}$ percentile of the IEI values distribution for the given a priori ionosphere parameter error covariance matrix $M$. For this test case, the predicted mean error (green $\mathrm{X}$ ) and the mean of the Monte Carlo generated errors (red cross) are 10 meters apart. It is worth noting that such differences between predicted and Monte-Carlo-generated mean error values have only been observed with very large IEI values, as with this test case. This fact suggests that the most challenging nonlinearities are those of the ionosphere model and the ray-path solver that uses the model. Fifty one out of 200 points fall outside the theoretical $90 \%$ error ellipse for the horizontal plot, and 60 out of 200 points fall outside the $90 \%$ error ellipse for the vertical plot. These large numbers demonstrate that the linearized covariance analysis is less reliable when large ionosphere model uncertainties 
lead to significant nonlinearities in the measurement model. Note, however, that the extent of the point spread about the Monte-Carlo mean is well modeled by the theoretical $90 \%$ error ellipse. The statistical mis-match is caused almost entirely by the mismodeling of the mean value due to ionosphere/ray-tracing model nonlinearities.

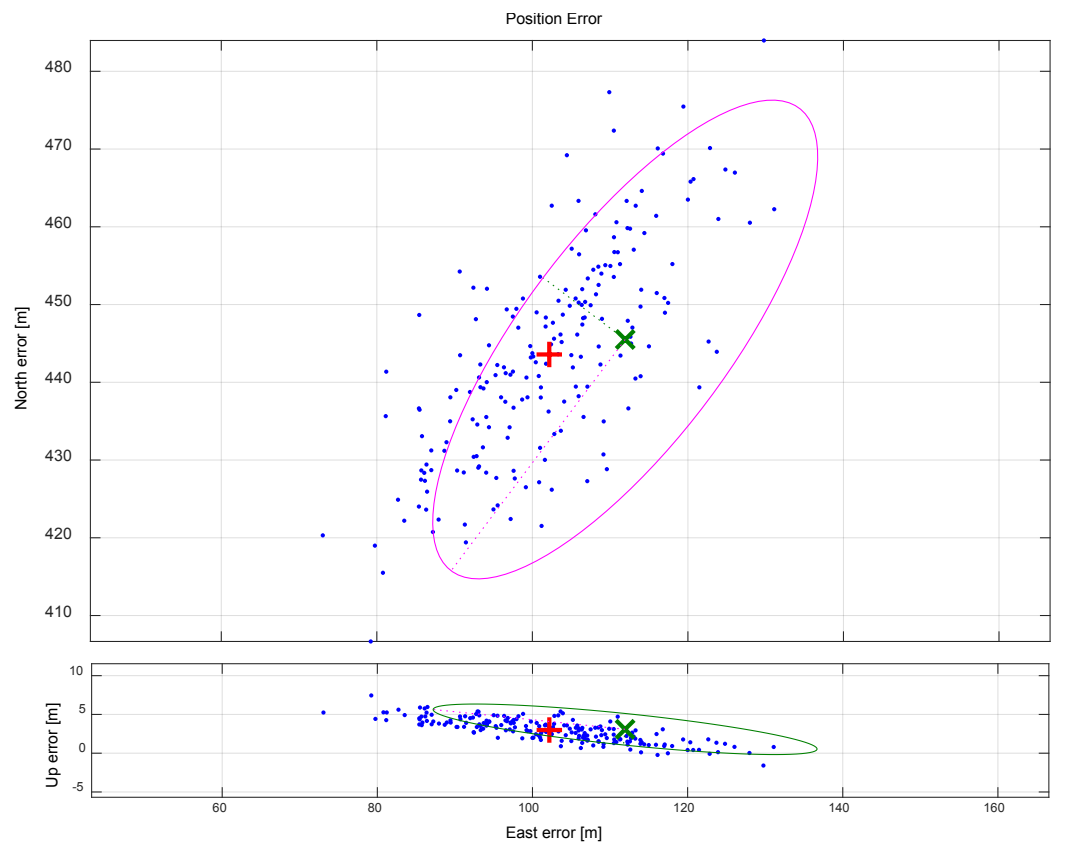

Figure 22: Empirical and theoretical position error distributions for Test Case 3531. Agreement between the two analyses is demonstrated for this setup that is characterized with a very significant error in the a priori parameterized ionosphere model. In this plot, the $90 \%$ error ellipses are centered about the theoretical mean.

For Test Case 3611B (Fig. 23), a 10-meter standard deviation for the measurement errors has been used, but the a priori IEI is as small as for Test Case 3511. Eighteen of 200 points fall outside the relatively large horizontal error ellipse, and 16 out of 200 points fall outside the vertical error ellipse. These numbers demonstrate consistency for this test case as well. 


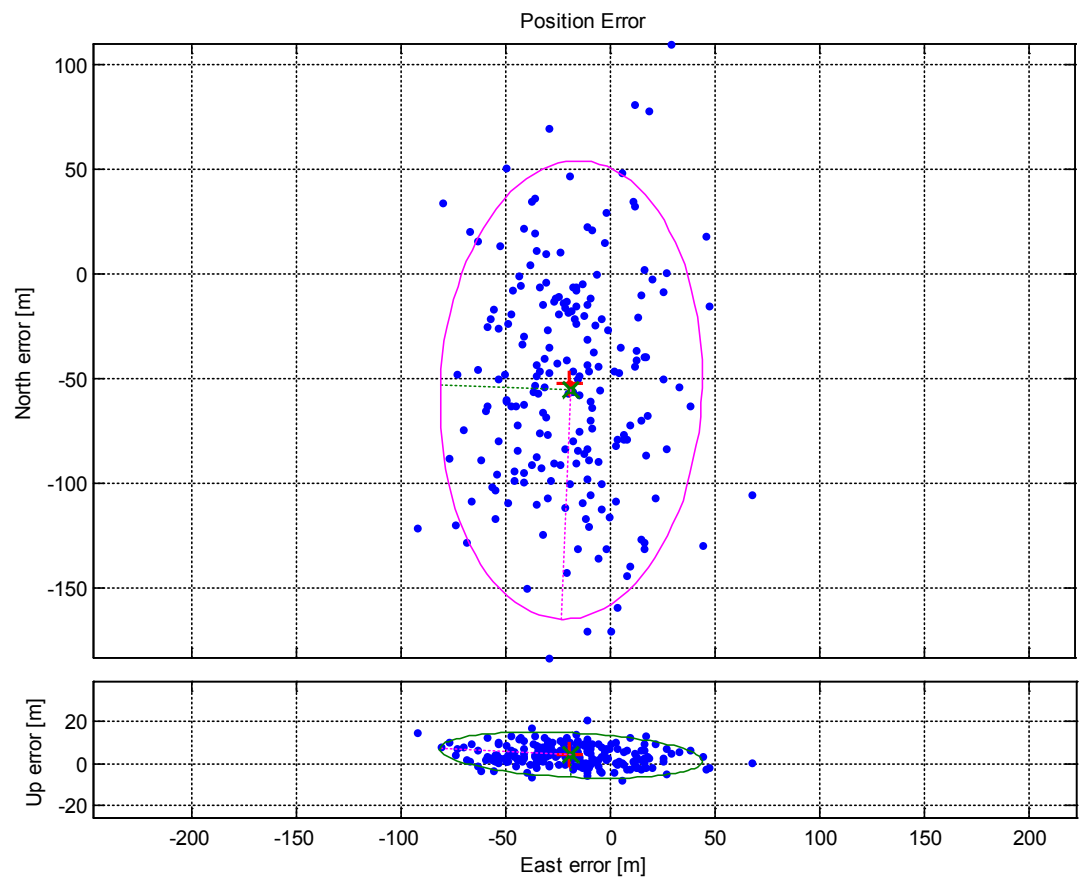

Figure 23: Empirical and theoretical position error distributions for Test Case 3611B for which much larger measurement errors were assumed. With 18 of 200 points falling outside the horizontal error ellipse and 16 out of 200 points falling outside the vertical error ellipse, consistency is demonstrated for this test case. 


\section{CHAPTER 7}

\section{RESULTS FOR BATCH-FILTERING TEST CASES}

\subsection{Previous Results}

The study of Ref. [5] considered the simplified, straight-segments, ionosphere-thinsheet-reflections ray-path model that has been described in Section 5.1. Nine test cases were studied in that work. These test cases were characterized with setups that differed in number of ray-paths, true and a priori ionosphere models, and ground station placement. The study was carried out in the scope of a limited number of executions. Preliminary results suggested that the simplified-model problem presented in that paper is sufficiently observable to make such a system a candidate for navigation.

Position errors, ranging from tens to thousands of meters, appeared to be consistent with the corresponding computed Cramér-Rao bounds. At the same time, the filtered estimates of the ionosphere electron density profile parameters tended to have significantly reduced errors in comparison to the a priori models. It was concluded, therefore, that the method discussed may also be useful for remote-sensing-based ionosphere characterization in cases where the receiver location is known a priori.

Improved results were typically observed when more measurements were available. Improved results also tended to occur when the uncertainties of the a priori ionosphere model decreased. With only a limited number of test cases analyzed, the apparent trends in simulated errors were less significant than trends in the computed Cramer-Rao bounds. 
It was concluded that the number of received signals (ray-paths) is expected to have a significant impact on the system's performance. At the same time, it was also observed that positioning accuracy is sensitive to the exact setup used for a particular test case, and it became harder to make general predictions about accuracy as the number of received ray-paths decreased.

The beneficial impact of having a wide range of signal frequencies was also evident when variations of the test cases were tested with a limited range of signal frequencies. It was also observed that the errors in the final positioning estimates were only loosely related to the errors in the a priori ionosphere model.

Further investigations have shown that in all cases the solutions for the simplified minimization problem appeared to have converged to their global minimum. Thus, the presence of nonlinearities in the model does not seem to pose a significant challenge to solving the underlying batch estimation problem. 


\subsection{An Overview of Methodology}

Previous chapters introduced the various aspects of an HF navigation system that relies on signals transmitted from ground stations. A significant part of the assessment of this project's feasibility consists of an evaluation of its performances in terms of positioning accuracy, as well as in its ability to effectively apply corrections to an erroneous a priori model of the ionosphere.

Assessment is performed through a comprehensive analysis of a series of test cases that differ in the sets of parameters that define them. These parameters include the following: type of available measurements, number of ground stations and their placement, number of ray-paths, ray-path geometry, the number of hops for each raypath, signal frequencies, true and a priori ionospheric models, receiver clock error, and the true location of the receiver.

\subsubsection{Simulation-based analysis}

The primary portion of this analysis is based on four classes of test cases. The first two, Classes 1 and 2, consist of well-defined test cases which are characterized by constant true and a priori ionosphere models. Input parameters or sets of parameters are altered one at a time, thus yielding an ensemble of variations of a base test case. Results for these test cases are assessed through examination of executions of the batch-filtering algorithm on the simulated data in either Nominal Mode or Performance Analysis Mode. In the latter case, statistics are generally obtained by processing the outcome of sets of Monte-Carlo runs. This approach is useful in assessing the batch-filtering algorithm's 
performance given particular setups and IEI values, and as such, it provides in-depth, yet somewhat narrow-scope information.

As demonstrated before, the likelihood for encountering the particular IEI values that are considered with a given test case can be evaluated based on the histogram of Fig. 2 . However, it is important to recognize that this methodology does not make any assumptions on how a priori ionospheric model errors are distributed and therefore it is expected to deliver insightful results within its scope.

The drawback with this method is its relatively narrow scope in terms of ionosphere modeling, as only several different erroneous a priori ionosphere models are considered with each class of test cases.

\subsubsection{The statistical analysis for a random-ionosphere model}

The drawback of considering only a few error models in the a priori ionosphere model provides the motivation for conducting a study of a second type that relies on statistics which are obtained for a random-ionospheric-errors model, so that ionosphere model errors' randomness is accounted for. This second type of analysis assumes that the difference between the true and a priori ionosphere parameter vectors is a random vector sampled from a zero-mean Gaussian distribution. While a Gaussian model for these errors may seem an obvious choice, two important aspects of this approach should be recognized. Examining the bottom plot of Fig. 2, it can be inferred that the random

variable $10^{\xi}$ is not Chi-Square distributed and therefore $\underline{\varepsilon}_{p}$ is not a sample from a multivariable Gaussian distribution. It has been left to a future study to determine to what 
fidelity can ionospheric errors be modeled with a Gaussian model. Even if the distribution is not Gaussian, its second moment, its covariance, is still properly analyzed by the techniques of this statistical analysis if the effects of the measurements and the batch estimation are well approximated by a linearized model.

A second potential pitfall of the Gaussian ionosphere error assumption concerns the feasibility of setups that have been generated with random errors that are added to the Chapman ionosphere parameters. It has been observed with simulated executions of the batch-filtering algorithm, that, depending on the covariance matrix that has been used to generate the errors for the ionospheric model, ionosphere setups are prone to physical non-feasibility. For the test case used in Subsection 6.5.1 that describes covariance analysis validation for the case of random ionosphere errors, with $M_{0}$ factored by 0.1 , two percent of the Monte Carlo runs failed the physical feasibility test and consequently declared non-feasible. For a non-scaled-down $M_{0}$, however, 92 percent of the Monte Carlo runs failed the test. These results imply that a linearized model of the effects of ionosphere errors on the batch filter errors, as in Eq. (99), breaks down for $M=M_{0}$, but that it is reasonable for $M=0.1 M_{0}$. Despite this limitation, this analysis will be used because it is the simplest way to get a handle on the likely effects of an entire statistical ensemble of possible ionosphere errors. At the same time, one must keep in mind that the predicted filter errors for the largest levels of ionosphere modeling error covariance are probably not modeled very well by this analysis technique.

Therefore, it is presumed that modeling $\underline{\varepsilon} p$ with a Gaussian model is an approximation that, under certain assumptions on $M$, will yield reasonably reliable results for the 
purpose of statistical analysis. A model is considered where $\underline{\varepsilon}_{p}$ is assumed to be a sample from a Gaussian distribution which is represented by a scaled covariance matrix $M_{0}$. This matrix, which was first introduced in Section 2.3.1, can be regarded as a measure of the diversity of the a priori ionosphere spatial electron density profile. When scaled by the constant $\gamma$, the resulting covariance matrix, $\gamma M_{0}$, can be used to generate scenarios of low (small $\gamma$ ) and high (large $\gamma$ ) uncertainties for the a priori ionosphere model.

The procedure for the statistical analysis of the a posteriori ionosphere estimation errors is as follows. First, 1000 random error vectors are generated based on $M$ and $R$, such that each set of error terms contains the appropriate number of parameters for the ionosphere model, which depends on the number of applicable grid nodes, and the appropriate number of measurement errors, which depends on the number of considered ray-paths. Next, the a posteriori estimation errors are computed for each set of error terms using the linear approximation of Eq. (99). The a priori and a posteriori ionospheric parameter vector estimation errors are then added to $\underline{p}_{\text {true }}$ to produce the $a$ priori and a posteriori ionosphere estimates. In the next step, all 1000 sets of a priori and a posteriori estimates for the ionosphere model are used to generate six Chapman parameter maps of the kind that will be presented later, starting with Fig. 25. Differencing these maps from maps of the three true Chapman parameters of the same kind yields 1000 error maps for each a priori Chapman parameter and similarly, 1000 error maps for each a posteriori Chapman parameter. These maps are statistically processed to generate six maps for $80^{\text {th }}$ percentile values of the 1000 maps. The procedure is repeated for five different values of $\gamma$, bringing the total number of $80^{\text {th }}$ - 
percentile-values maps for each test case to 30 . The five $\gamma$ values are $1,0.5,0.1,0.001$, $10^{-9}$. The case of $\gamma=1$ corresponds to a very significant uncertainty for the $a$ priori ionosphere model, whereas $\gamma=10^{-9}$ implies an almost perfect a priori model for the ionosphere.

A second procedure is carried out to produce position error plots of the type that is presented starting Fig. 57. The dimensions of the horizontal 90\% error ellipse and of the vertical $90 \%$ error ellipse are calculated based on a covariance matrix which is calculated using Eq. (100). As with the calculations for the ionosphere parameters, this procedure considers the five different scaling factors $\gamma$ that multiply $M$ in Eq. (100).

\subsubsection{Test case groups and test case classes}

Several classes of test cases are considered. Class 1 consists of fixed-ionosphere scenarios where only group delay measurements are processed. These test cases are grouped into three groups - A, B and C - that have setups with 21, 33 and 128 available measurements, respectively.

The second class of test cases studied in this analysis, Class 2, considers fixedionosphere scenarios where both group delays and beat carrier phase measurements are processed. These test cases are grouped into two groups - D and E - that have setups with 17 and 33 ray-paths, respectively. It should be noted that for dual-measurement-type test cases of Class 2, each ray-path is assumed to be sampled at four different times that are very close to each other, so that each ray-path is sampled with four different carrier frequencies in a way that yields beat carrier phases with a common bias as measured in 
carrier cycles. This brings the total number of processed measurements for these test cases to 8 times the number of ray-paths.

Class 3's test cases of Group F are identical to Those of Class 1's Group C except that, consistent with the statistical methodology, they consider a random ionosphere error model rather than a fixed error model. The included test cases are therefore studied with the statistics-based method only. As with Class 1's test cases, analysis is performed under the assumption that only group delay measurements are processed.

Similarly, Class 4's Groups $\mathrm{G}$ and $\mathrm{H}$ are the statistical analysis equivalents to Class 2's Groups D and F. With these test cases, both group delays and beat carrier phase measurements are processed

\subsubsection{Chapter scope}

This chapter presents the results that have been obtained for the many different test cases that have been studied. It additionally provides the required tools for comparing different types of data that have been obtained, observed, or computed for the various test cases. The presented results will be reviewed and further analyzed in the next chapter.

As the previous subsection implies, the following four sections - 7.3-7.6 - contain a significant amount of information. As a user's roadmap, both test cases' setups and primary results are summarized in the form of tables at the beginning and the end of each section, for the sake of convenience. However, the reader should bear in mind that these tables do not include all information presented in the text. 


\subsection{Test Cases Using Group Delay Only, Fixed Ionosphere Model (Class 1)}

The purpose of this section is to present the performance of the system for the case where only group delay measurements are processed. Table 1 , which can be regarded as a roadmap to this section, summarizes the primary characteristics for all eight test cases analyzed in this section and its subsections, the cases designated $\mathrm{A} 0, \mathrm{~B} 0-\mathrm{B} 4, \mathrm{C} 0$, and C4. The table summarizes their measurement noise's standard deviation, number of raypaths, initial IEI, direction of signals as they arrive at the receiver (above the horizon or both above and below) and the placement pattern for the ground stations from which received signals are broadcasted. The three groups of test cases of Class 1 correspond with three scenarios of low, medium, and high numbers of available signals/measurements, designated as groups $\mathrm{A}, \mathrm{B}$, and $\mathrm{C}$, respectively.

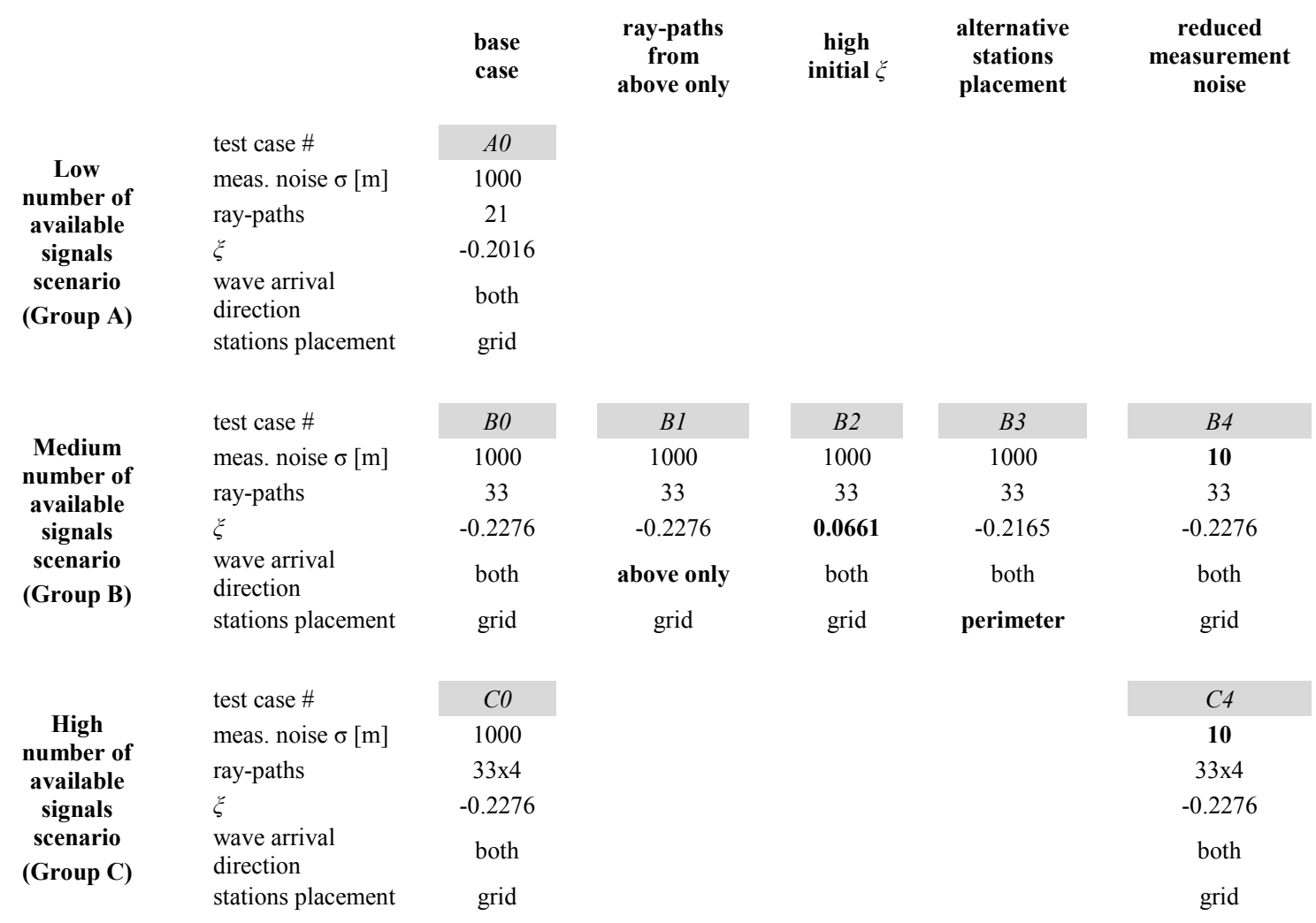

Table 1: Setup configuration for Class 1's three groups and eight test cases. 
For each group, a base test case is evaluated first. These are Test cases A0, B0, and C0. Sensitivities to the setup's parameters are examined next through an analysis of variants of those base test cases. These variants include test cases with altered ray-paths geometry (the column labeled ray-paths from above only), test cases that are characterized with significantly higher $\xi$ values, i.e., larger differences between the truth and a priori ionosphere models (column labeled high initial $\xi$ ), test cases with an alternative placement of ground stations (column labeled alternative stations placement), and test cases with reduced measurements noise (right-most column). In order to maximize comparability, all test cases have the same true parameterized ionosphere model.

Each test case is evaluated by two methods: (a) Its performance as obtained for its execution in Nominal Mode, as defined in Subsection 6.1.1, and (b) An evaluation of its performance through a Monte Carlo analysis, using the Performance Analysis Mode.

The first method is useful for testing cases' feasibility, assessing the filter's performance in correcting the ionosphere model through latitude/longitude mapping of both a priori and a posteriori ionosphere parameter error maps, and for obtaining theoretical errors and error distribution assessments. The second method is performed in a manner that is aligned with the setup described in Subsection 4.4.2, which considers constant a priori ionosphere errors and random ranging measurements errors. This approach is useful when analyzing the impact on performance of altering the different setup parameters. 
These analyses will additionally enable a later discussion about batch filtering performance given 'easy' and 'hard' scenarios that differ in their a priori IEI values. 'Easy' scenarios have IEI values that lie on the left side of the histogram that is presented in the bottom panel of Fig. 2. Similarly, 'hard' scenarios have IEI values that lie on the right side of that plot. An assessment on occurrence likelihood associated with particular a priori IEI values can be obtained based on the data presented on that histogram.

Measurement errors were generated using a Gaussian, zero mean distribution with a 1sigma value of 1000 meters (except for reduced-measurement-noise test cases). This level of accuracy is about the best that can be achieved with code-based measurements due to the signal's limited bandwidth, as mentioned in Subsection 2.1.1. A second model with a standard deviation of 10 meters was used in order to assess the sensitivity to ranging precision.

\subsubsection{Test cases with a medium number of available ray-paths (Group B)}

All test cases of Group B consider a set of eleven ground station transmitters at various locations across the Contiguous United States (CONUS). This set of ground transmitter stations is a subset of an array of ground station transmitters that is based on a grid of small circles of constant latitude, spaced 5 degrees apart. The longitudinal difference between two neighboring stations that lie on the same small circle is 10 degrees. The longitudes of stations that lie on two neighboring small circles are offset by 5 degrees. Note that a typical test case will consider signals that are received from only a subset of this set of ground station transmitters, hence the missing transmitters from this regular grid in the example shown in Fig. 24. The user receiver is located at 
latitude/longitude/altitude (LLA) $\left[40.1^{0},-95.1^{0}, 10000 \mathrm{~m}\right]$, i.e., at the center of the green, spider-like object in Fig. 24. Note that the symbol definitions for ground stations, ray paths, etc. used in Fig. 24 are the same as have already been discussed in connection with Fig. 7. The user receiver receives three signals from each ground station, which results in a total of 33 ray-paths. The figure's curved trajectories of the true ray-hops in green reflect their refractive nature. These trajectories have been computed by the raytracing engine. The reader may want to compare this plot with the equivalent plot on Fig. 1 of Ref. [5] in which the segmented nature of ray-paths that corresponds to the simplified signal propagation model is evident.

Test Case B0 is regarded as the base test case for the medium number of available raypaths scenario. All other test cases in Group B are variants on this test case that are used to assess performance sensitivity to the various parameter sets that define the scenarios. For this test case, the truth ionosphere electron density profile is based on the IRI model computed for October 23, 2009, at UTC 14:22. The a priori model is based on the model computed for September 23, 2009, at the same hour, such that the total seasonal discrepancy is one month, and the corresponding Ionosphere Error Index $\xi$ is -0.2276 for the a priori model. The statistical analysis in Section 2.3.1, indicates that this $\xi$ value lies within the $65^{\text {th }}$ percentile of the IEI values distribution for the given a priori ionosphere parameter error covariance matrix $M$. The HF signals for this test case have frequencies in the range 4.2-5.8 MHz. The number of hops for each ray-path is $1-4$, with a mixture of signals arriving from above the user equipment (UE) and from below. 
This test case was first run in a Nominal Scenario Mode, i.e., with pseudorange measurements that are identical to the true range-equivalent group delays. A close look at the estimated values indicates that the final position error in this case is -197 meters in the local north direction, -104 meters in the local east direction, and -24 meters in the vertical direction. The error in the estimate of the receiver clock bias is equivalent to 15 meters. This result is consistent with the analytically predicted position error of -199 meters in the north direction and -101 meters in the local east direction, where predication is performed using the right-most term of Eq. (99).

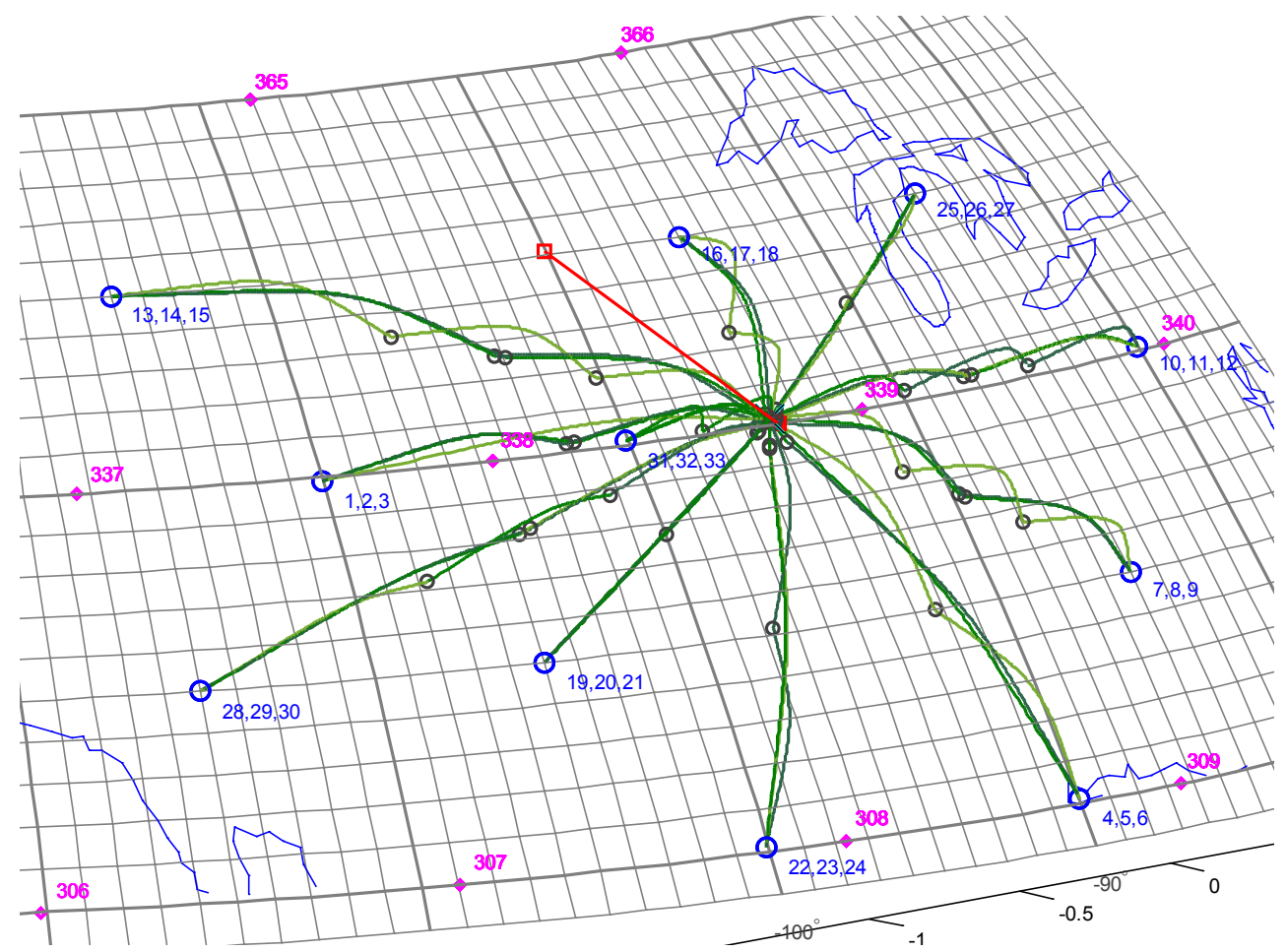

Figure 24: Setup for test cases of Group B, ground stations (blue circles), true ray-path trajectories (green curves), and position solution convergence trajectory (red curve) for Test Case B0.

Figure 25 plots errors from truth for the a priori (top) and the a posteriori (bottom) estimates of the ionospheric peak electron density altitude parameter $h_{\max }$. These errors 
have been computed and plotted for a region that contains all active grid nodes for this test case. In other words, this is the region where the ionosphere has been probed by the propagating signals. Other regions of the plot have been left blank/white. The red square at latitude/longitude $\left(40.1^{0},-95.1^{0}\right)$ indicates the true position of the receiver. Blue circles with white edges denote the locations of the ground stations. Magenta diamonds denote the locations of the bi-quintic spline grid nodes. The small green squares mark computed truth Earth bounce-points. North America's coastline is shown in white with the borders of the states shown in gray. It is evident that the initial errors in $h_{\max }$ have been reduced dramatically above the vast majority of CONUS. For the a priori data, $80 \%$ of errors above CONUS are below $14.1 \mathrm{~km}$ and $95 \%$ are below $20.2 \mathrm{~km}$. For the a posteriori (or estimated) model, $80 \%$ of errors are below $2.1 \mathrm{~km}$ and $95 \%$ are below $3.6 \mathrm{~km}$. At the same time, significant errors for the ionospheric scale height parameter $h_{\mathrm{sf}}$ of $6.7 \mathrm{~km}$ and $7.7 \mathrm{~km}$ have been reduced to $1.2 \mathrm{~km}$ and $2.8 \mathrm{~km}$ for the $80^{\text {th }}$ and $95^{\text {th }}$ percentiles, respectively, as demonstrated in Fig. 26.

A significant reduction in errors for the VTEC parameter has also been achieved, as shown on Fig. 27, from 1.24 TECU to 0.16 TECU for the $80^{\text {th }}$ percentile and from 1.92 TECU to 0.21 TECU for the $95^{\text {th }}$ percentile. Note, however, that for the given lengthequivalent group delay measurements, electron density errors are observable only at heights that are less than the height for which maximal electron density is obtained, i.e. $h_{\text {max. }}$ In other words, the observable part of VTEC is only that part of the electron density integral up to the altitude of peak density. This caveat holds true for all of the VTEC accuracy results discussed in this dissertation. 

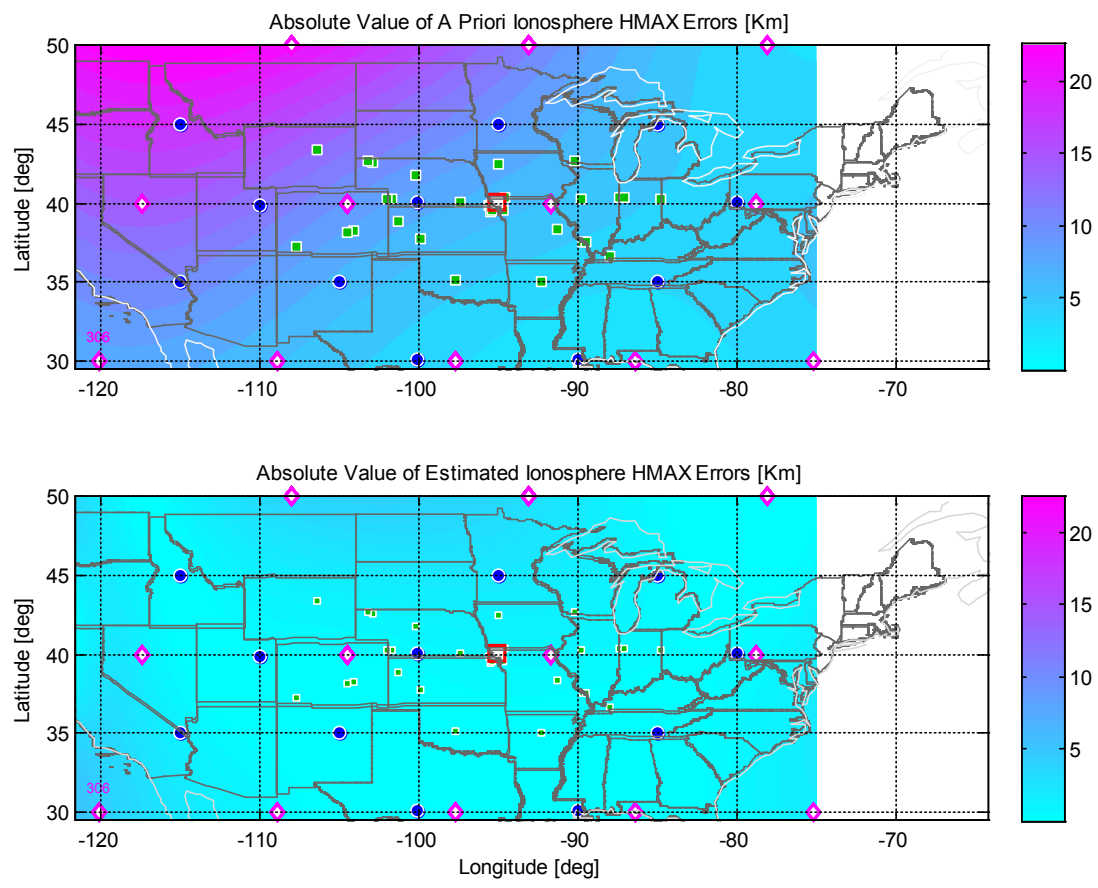

Figure 25: A priori (top) and a posteriori (bottom) errors for the ionospheric peak electron density height parameter $h_{\max }$ for a Nominal Scenario Mode run of Test Case B0.
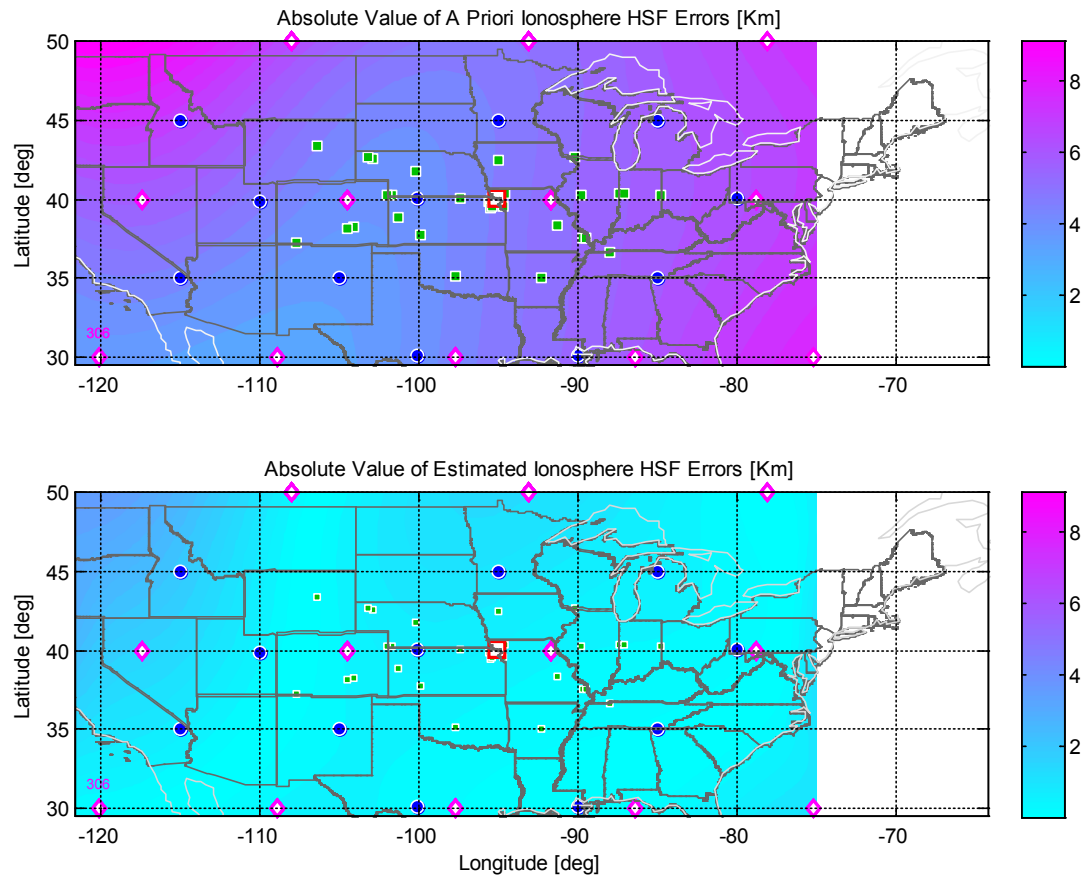

Figure 26: A priori (top) and a posteriori (bottom) errors for the ionospheric scale height parameter $h_{\mathrm{sf}}$ for a Nominal Scenario Mode run of Test Case B0. 
For the statistical analysis, Test Case B0 was next executed in Performance Analysis Mode with a 100-run Monte Carlo simulation and a 1-sigma measurement noise of 1000 meters that is aligned with the discussion of Subsection 7.2. Here, and throughout the rest of this chapter, each Monte Carlo run is characterized by a unique set of measurement errors.

The outcome of the execution is presented in Fig. 28 in the form of a scatter plot. The blue dots mark final estimation errors for the receiver's position, with a red cross marking their mean. The reference frame used in this plot is a local-level frame whose origin is located at the true location of the receiver. The magenta ellipse in the top plot is the $90 \%$ horizontal error ellipse that was obtained from the analytical error prediction. It can be verified that 9 dots lie outside this ellipse. The lengths of the ellipse's semi-major and semi-minor axes are approximately 1765 and 1347 meters, respectively. The standard deviation for the vertical position error is 311 meters. The green ellipse in the bottom plot is the $90 \%$ error ellipse in the east-vertical plane that is also given by analysis. Twelve blue dots fall outside this ellipse. Having nearly 10 of the 100 cases that fall outside the two 90\% error ellipses in Fig. 28 demonstrate that the linearized error analysis associated with Eqs. (99) and (102) is reasonably accurate. Recall that this analysis assumes a constant ionosphere parameter error, which induces a bias via the final term on the righthand side of Eq.(99), and that the statistical variations of the navigation solutions about this bias are caused by random pseudorange measurement noise and are modeled by the covariance analysis in Eq. (102). 

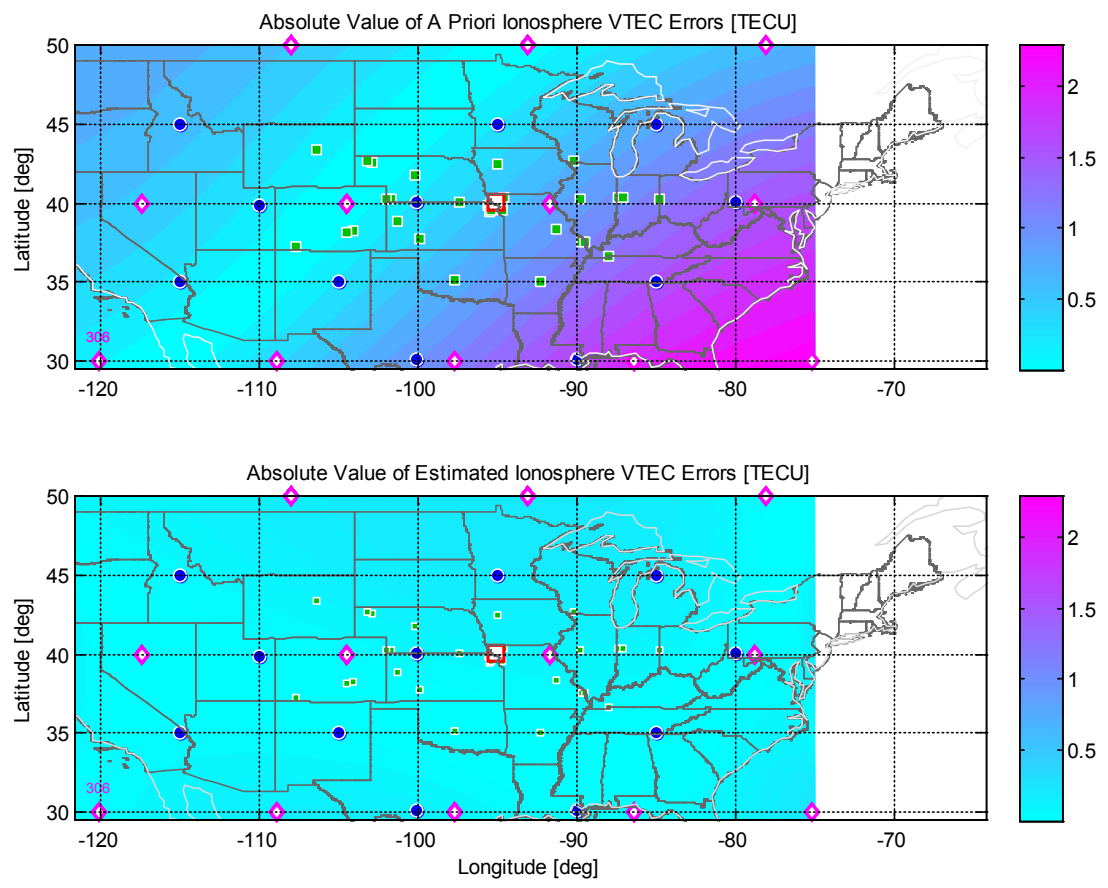

Figure 27: A priori (top) and a posteriori (bottom) errors for the ionospheric vertical total electron content parameter VTEC for a Nominal Scenario Mode run of Test Case B0.
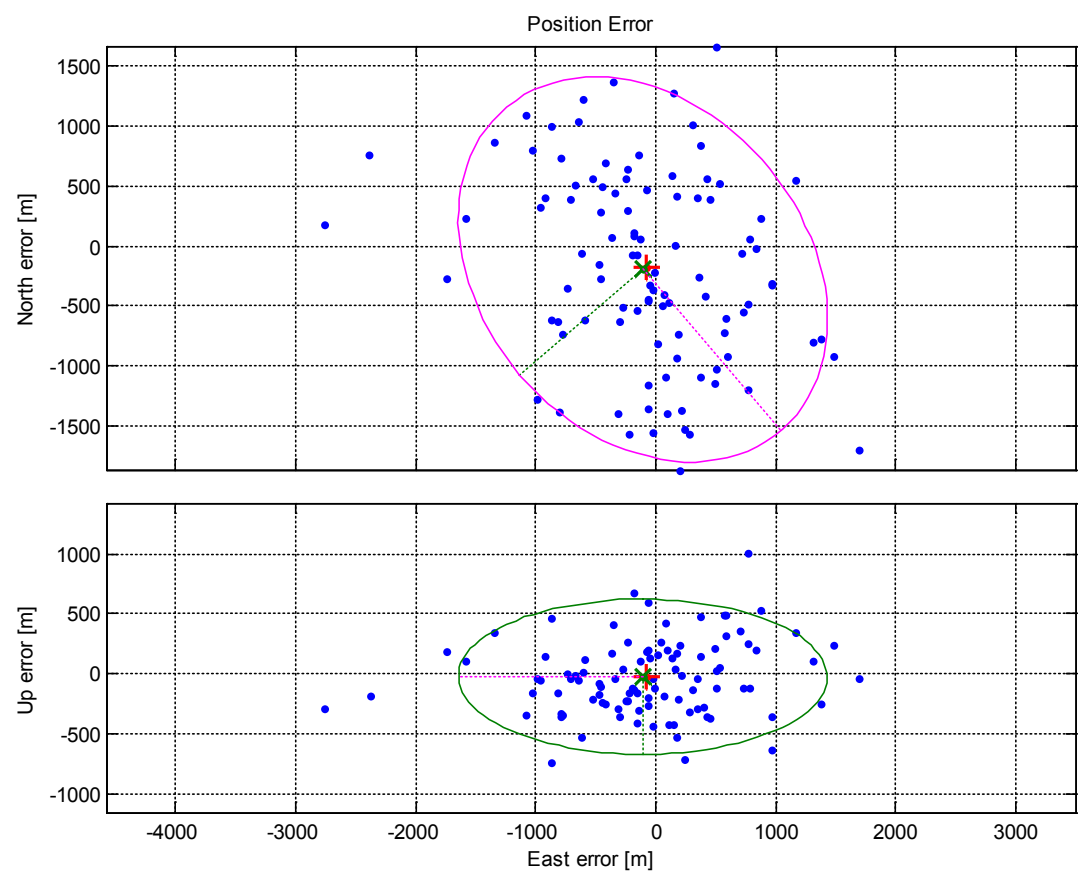

Figure 28: Position error pattern for Test Case B0; Monte Carlo analysis scatter plot (blue); Horizontal 90\% errors ellipse (top plot, magenta); Vertical 90\% errors ellipse (bottom plot, green). 
The statistical properties of the a posteriori ionosphere model errors are shown in Fig. 29. This figure should be interpreted as follows: The top panel includes two histograms for the $h_{\max }$ parameter's residuals. These histograms reflect the statistical distribution of the a posteriori $h_{\max }$ errors' $80^{\text {th }}$ and $95^{\text {th }}$ percentiles values. The two sets of values have been calculated for all 100 Monte Carlo runs. For example, by considering the $80^{\text {th }}$ percentile histogram in the top panel of Fig. 29, one can infer that in $36+8=44$ of 100 Monte Carlo runs, a posteriori $h_{\max }$ errors had values of $2.66 \mathrm{~km}$ or less over at least $80 \%$ of the area above CONUS, and in 96 out of 100 cases a posteriori $h_{\max }$ errors had values of 7.66 or less over at least $80 \%$ of the area above CONUS.
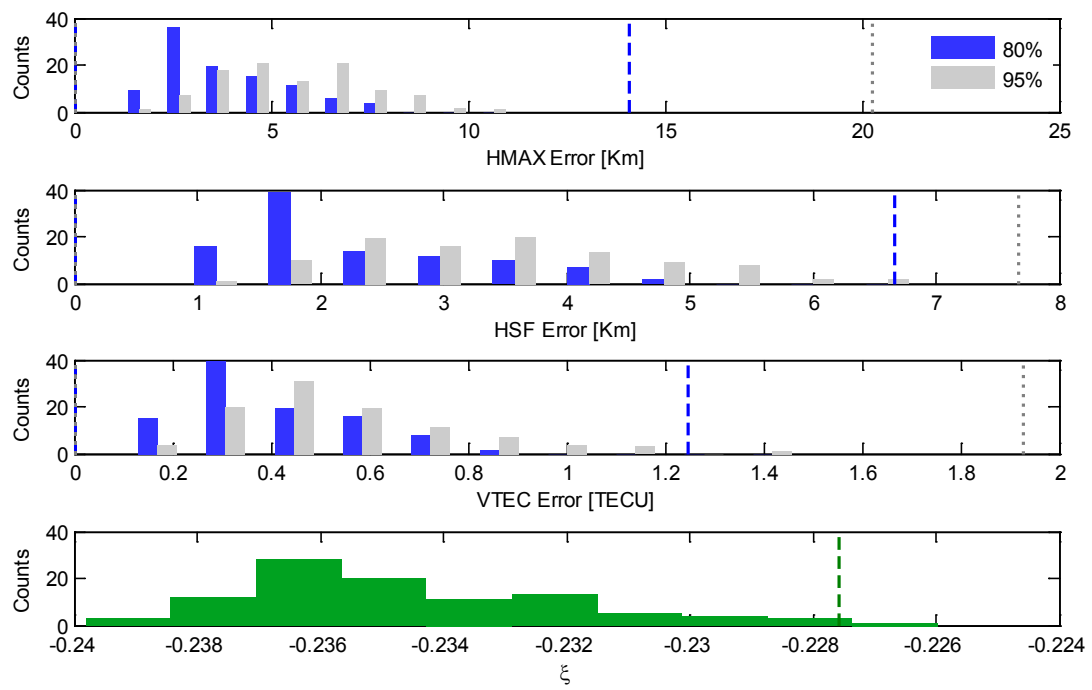

Figure 29: The statistical characteristics of the a posteriori ionosphere model errors for Test Case B0. The top three panels show histograms of the $80^{\text {th }}$ and $95^{\text {th }}$ CONUS area percentile error limits for the three Chapman parameters. The bottom panel is a histogram of $\xi$ values. Dashed lines mark the $80^{\text {th }}$ and $95^{\text {th }}$ percentile values of these quantities for the $a$ priori ionosphere parameter error vector, except in the bottom panel the dashed line marks the a priori error's mean. 
Similarly for the $95^{\text {th }}$ percentile, in 59 of 100 runs, $h_{\max }$ errors had values of 6.66 or less over at least $95 \%$ of the area above CONUS. In 97 of 100 runs, $h_{\max }$ errors had values of 8.66 or less over at least $95 \%$ of the area above CONUS.

The blue dashed line and the gray dotted line mark the a priori ionosphere model error values for the $80^{\text {th }}$ and $95^{\text {th }}$ percentiles, respectively. These reference values, applying for all 100 runs, were discussed earlier in the context of results obtained in Nominal Scenario Mode execution. These lines lie well to the right of their corresponding histograms in the top panel of Fig. 29, which indicates that the a posteriori estimates of the $h_{\max }$ map are much better than the a priori estimate.

The second and third panels of Fig. 29 plot the same type of information for the $h_{\mathrm{SF}}$ and VTEC Chapman model parameters. It can be inferred that the estimates for these two parameter maps have been improved for all 100 Monte Carlo runs by the fact that both plots have a posteriori histograms that lie well to the left of their corresponding a priori vertical lines. The bottom panel plots the distribution histogram for the a posteriori values of the IEI parameter $\xi$. The mean value equals -0.2340 which is smaller than the $a$ priori value of -0.2276 that is depicted by the vertical dashed green line (recall the logarithmic nature of this parameter). It can be also concluded that for two Monte Carlo runs, the a posteriori ionosphere model is in fact inferior to the a priori model. This does not constitute a discrepancy between the bottom plot and the top three plots because the IEI parameter that is considered in the bottom plot is computed from 27 parameters. It has been tested and shown that the three prime Chapman parameters may exhibit slightly different trends than the IEI. 
Test Case B1 is a variant on Test Case B0 that differs in its ray-path geometry. Here, ray-paths are configured such that all signals approach the receiver from above, i.e., the direction vectors of the incoming signals as they arrive at the UE have positive elevation above the horizon as viewed from the UE antenna. Minor modifications to the signals' frequencies were made in order to maintain the test case's physical feasibility. All other parameters were kept identical to those of the base configuration.

The positioning mean error for the nominal case (i.e., an execution in Nominal Scenario Mode) is 218 meters in the local north direction, -180 meters in the local east direction, and 73 meters in the vertical direction. This result is consistent to a 0.5 meters difference with the predicted error that was computed using Eq. (99). Results very similar to those obtained for the base Test Case B0 were obtained for the a posteriori estimates of the ionospheric parameters (plots are not shown). The Monte Carlo position errors plot of Fig. 30 reveals significant differences between test cases B0 and B1 in the geometric dispersion of the final errors. While the distribution of the scattered dots in the horizontal plane and its corresponding 90\% error ellipses have similar characteristics for the two test cases, errors in the vertical direction are substantially bigger for Test Case B1. For this test case, the major axis of the east-vertical $90 \%$ error ellipse that points almost vertically is 3875 meters in length compared to about 700 meters for the base test case of Fig. 28 . Interestingly, estimates for all three Chapman ionosphere parameters (Fig. 31) are slightly better for Test Case B1 in comparison to those of the base test case, B0. The average $80^{\text {th }}$ percentile values are about $3 \mathrm{~km}, 2 \mathrm{~km}$ and 0.35 TECU for Test Case B0's 

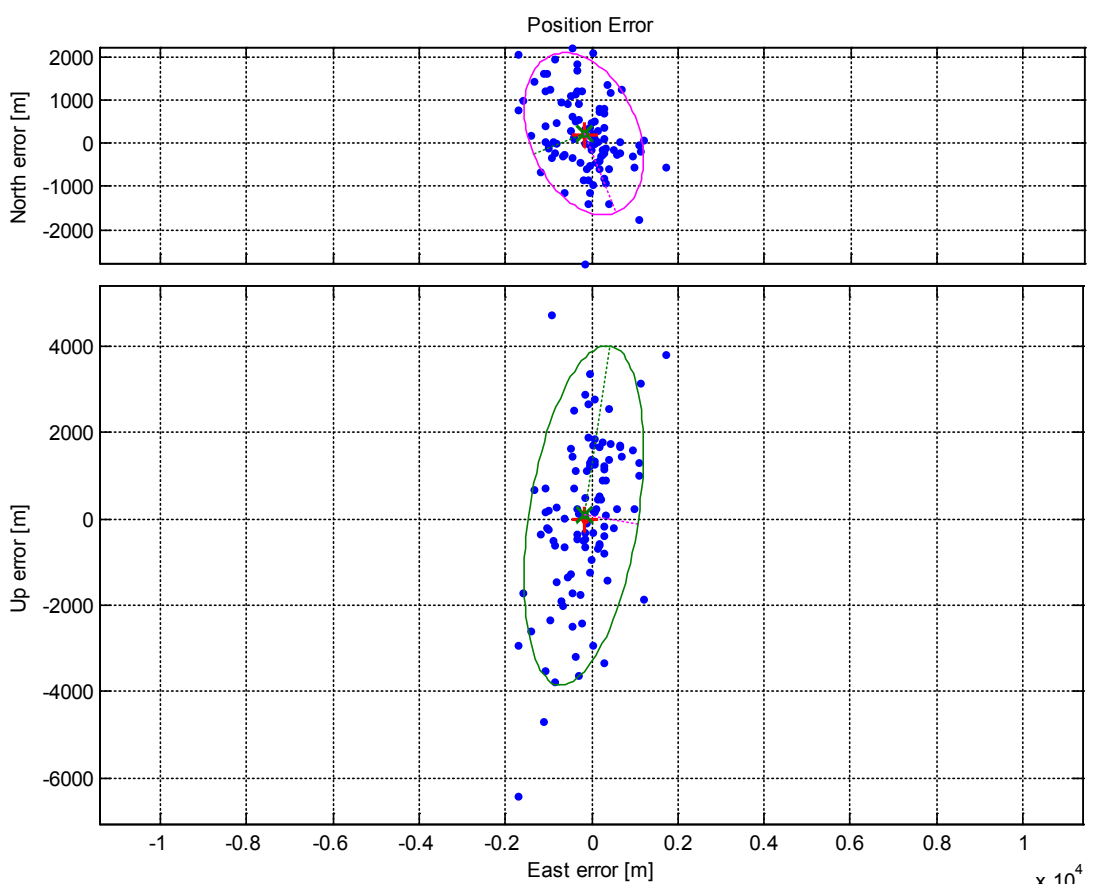

Figure 30: Position error pattern for Test Case B1; Monte Carlo analysis scatter plot (blue); Horizontal 90\% errors ellipse (top plot, magenta); Vertical 90\% errors ellipse (bottom plot, green).
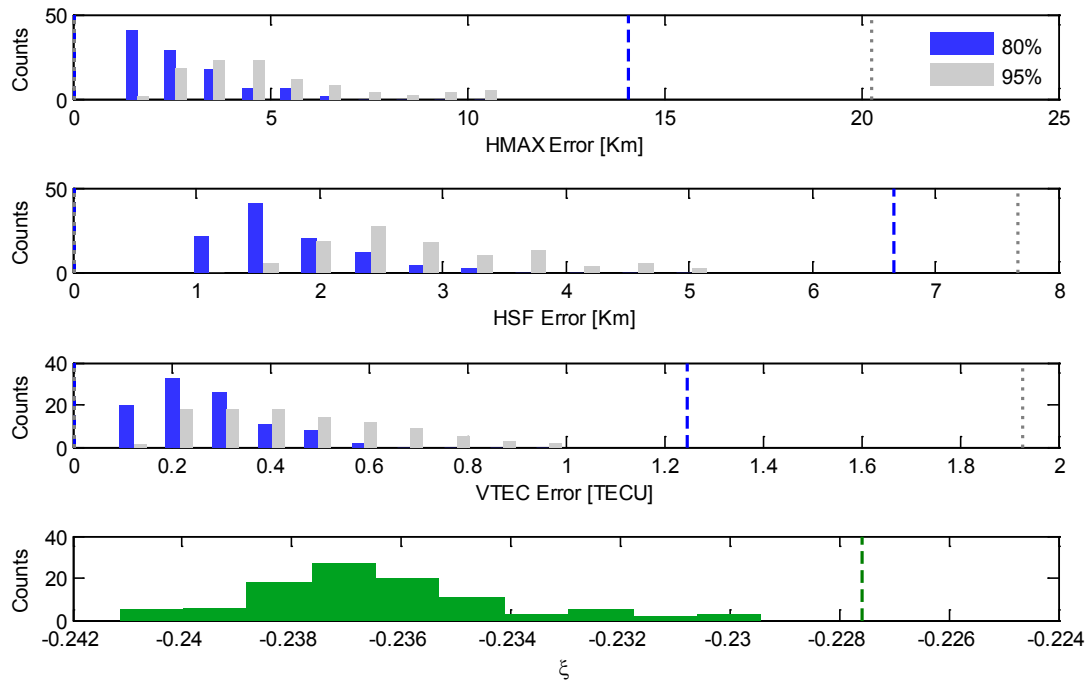

Figure 31: The statistical characteristics of the a posteriori ionosphere model errors for Test Case B1. The top three panels contain histograms of the $80^{\text {th }}$ and $95^{\text {th }}$ percentile error values for the three Chapman parameters. The bottom panel is a histogram of $\xi$ values. Dashed lines mark the $80^{\text {th }}$ and $95^{\text {th }}$ percentile values of these quantities for the a priori ionosphere parameter error vector, except in the bottom panel the dashed line marks the a priori error's mean. 
$h_{\max }, h_{\mathrm{sf}}$ and $V T E C$ parameters, respectively, whereas their Test Case B1's equivalent values are roughly $2 \mathrm{~km}, 1.5 \mathrm{~km}$, and $0.25 \mathrm{TECU}$.

Test Case $\mathrm{B} 2$ is a second variant on the base Test Case $\mathrm{B} 0$ that differs in its a priori ionospheric model. While the one month seasonal discrepancy of the base test case results in a computed a priori $\xi$ value of -0.2276 that lies in the $65^{\text {th }}$ percentile, the seasonal discrepancy of Test Case B2 was increased to three months, so that the truth ionosphere electron density profile is based on the IRI model computed for October 23, 2009 , at UTC 14:22, and the a priori model is based on the model computed for July 23 , 2009 , at the same hour. The resulting a priori $\xi$ value is -0.0661 and is located in the $95^{\text {th }}$ percentile of the IEI distribution histogram, as shown in the bottom panel of Fig. 2. All other parameters for this test case are identical to those of Test Case B0.

Equation (99) implies that bigger $\xi$ values are expected to result in bigger estimation errors for the nominal case, or equivalently, a bigger mean error for the full stochastic analysis that considers measurement errors. The position errors that have been computed for the nominal B2 case are 597, -269 and -50 meters in the local north, east, and vertical directions, respectively. Indeed, these errors are greater than the errors that have been computed for Test Case B0.

Results for the a posteriori estimation errors of the parameter $h_{\max }$ (Fig. 32) are consistent with the trend that is evident in inferior position errors. From a priori errors of $27.2 \mathrm{~km}$ and $46.3 \mathrm{~km}$ for the $80^{\text {th }}$ and $95^{\text {th }}$ percentiles of CONUS area, respectively, final estimation errors are $10.3 \mathrm{~km}$ for the $80^{\text {th }}$ percentile and $17.4 \mathrm{~km}$ for the $95^{\text {th }}$ percentile. These error statistics are significantly bigger than those for base Test Case B0. Similarly 
for the parameter $h_{\mathrm{sf}}$ errors that are shown in Fig. 33 , the $80^{\text {th }}$ and $95^{\text {th }}$ a posteriori estimates of $2.9 \mathrm{~km}$ and $3.5 \mathrm{~km}$ are worse than those obtained for Test Case B0. Still, errors have been reduced dramatically compared to the a priori values of $19.8 \mathrm{~km}$ and $21.1 \mathrm{~km}$ at the $80^{\text {th }}$ and $95^{\text {th }}$ percentiles of CONUS area.

Finally, VTEC values have been reduced from 1.71 TECU to 1.02 TECU for the $80^{\text {th }}$ percentile and from 2.69 TECU to $1.43 \mathrm{TECU}$ for the $95^{\text {th }}$ percentile (data not shown). These a posteriori error metrics are still significantly larger than their Test Case B0 counterparts.

Monte Carlo position error analysis results, shown in Fig. 34, exhibit characteristics that are very similar to those of Test Case B0 in terms of position error distribution about the mean. The main difference is in the magnitude of the mean error which is significantly further from zero for Test Case B2. Note how the Test Case B0's 90\% error ellipses are re-plotted in Fig. 34 and how their sizes are similar to those of Test Case B2 while their centers are closer to the origin than those for Test Case B2.

The impact of having significantly bigger a priori errors for the ionosphere parameters on the parameters' final estimates can be assessed by comparing the results shown in Fig. 35 with those of Fig. 29. In both cases, $80^{\text {th }}$ and $95^{\text {th }}$ error percentiles for the a posteriori ionosphere model have been reduced significantly from their a priori values. The $a$ posteriori estimates, however, are clearly superior for Test Case B0, for which errors are 2-4 times smaller than their Test Case B2 equivalents. This observation is based mainly on the computed means of the three Chapman parameters' histograms. However, for the 

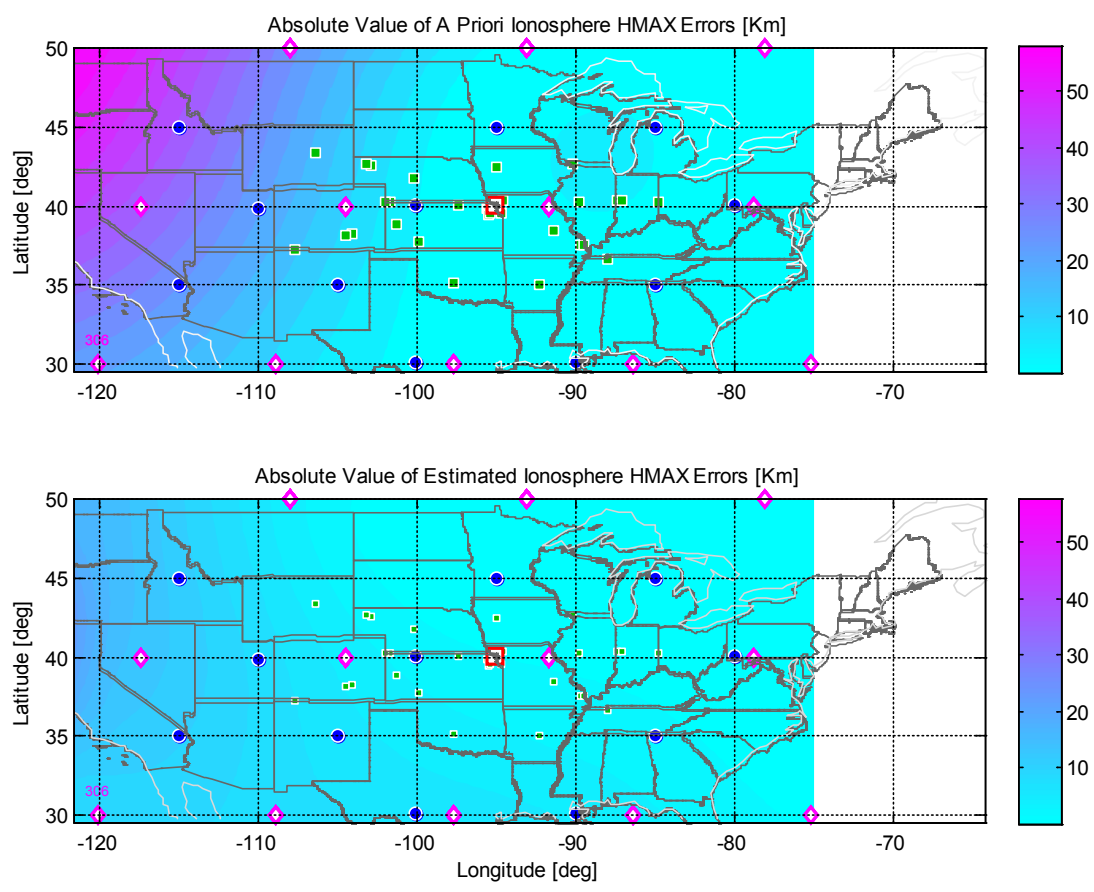

Figure 32: A priori (top) and a posteriori (bottom) errors for the ionospheric peak electron density height parameter $h_{\max }$ for Test Case $\mathrm{B} 2$.
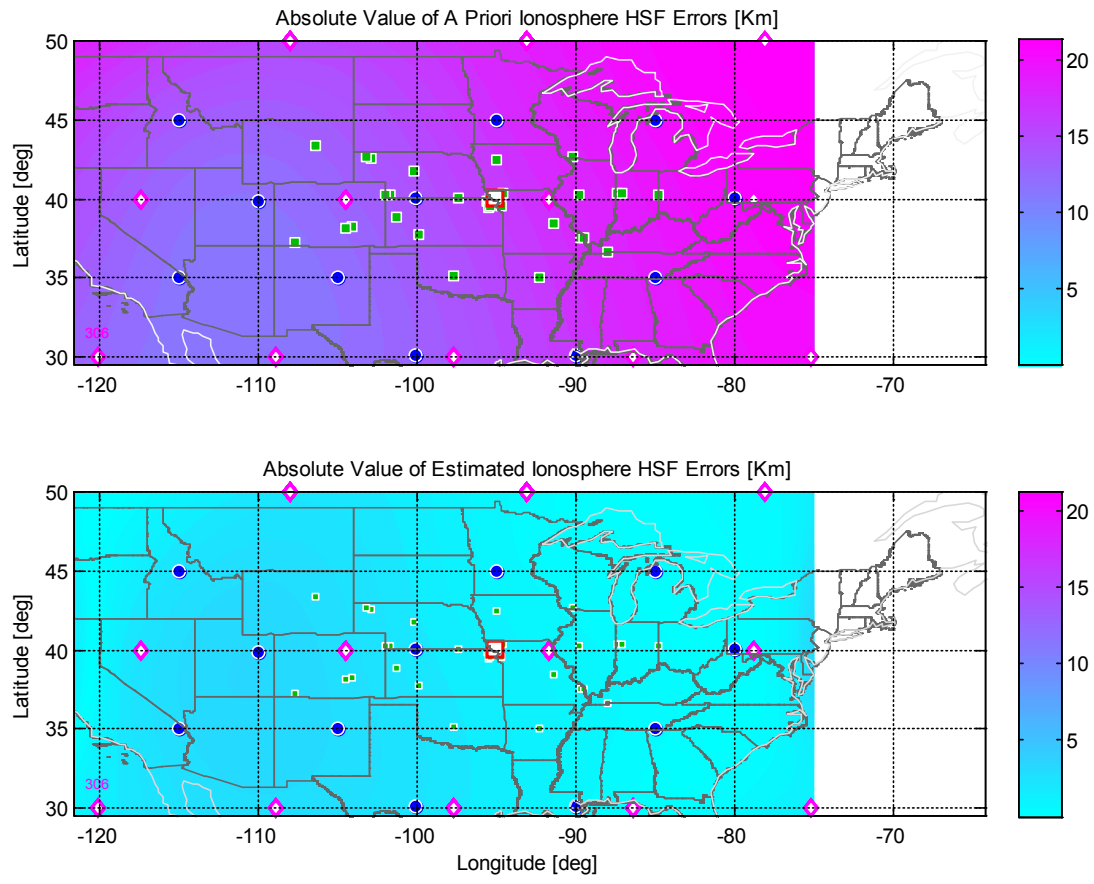

Figure 33: A priori (top) and a posteriori (bottom) errors for the ionospheric scale height parameter $h_{\mathrm{sf}}$ for Test Case B2. 

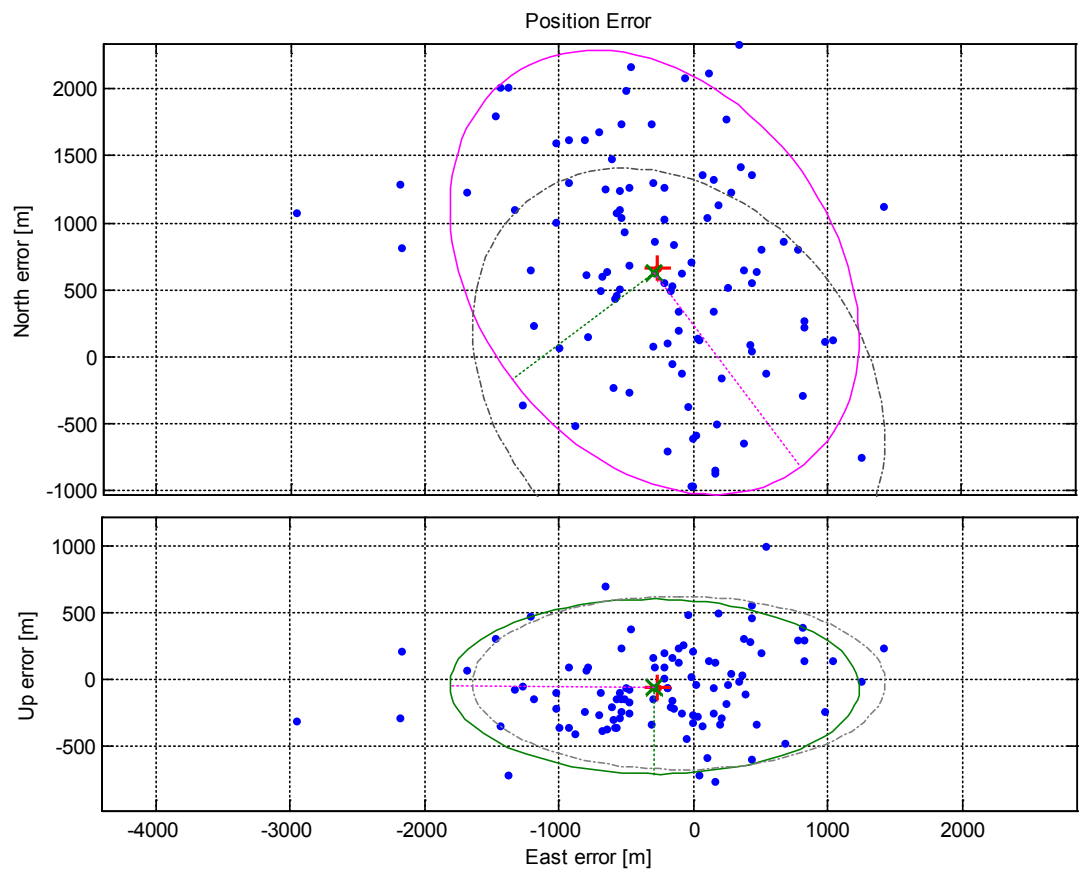

Figure 34: Position error pattern for Test Case B2; Monte Carlo analysis scatter plot (blue); Horizontal 90\% errors ellipse (top plot, magenta); Vertical 90\% errors ellipse (bottom plot, green); The dashed gray ellipses plot the $90 \%$ errors ellipses for base Test Case B0 and are shown here for comparison purposes.
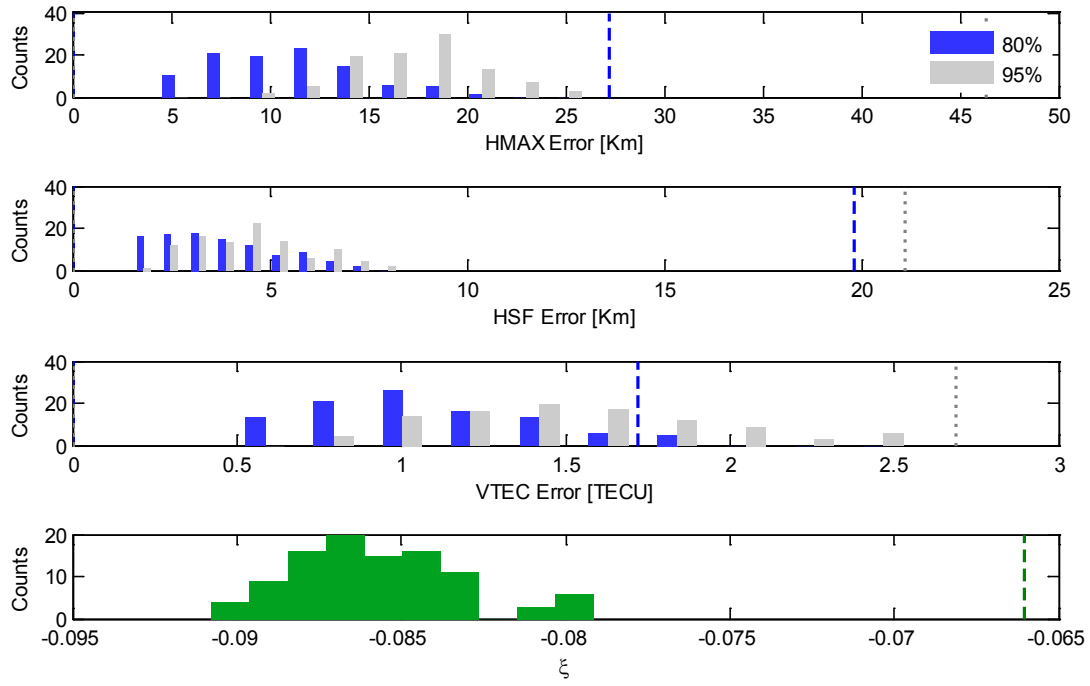

Figure 35: The statistical characteristics of the a posteriori ionosphere model errors for Test Case B2. The top three panels contain histograms of the $80^{\text {th }}$ and $95^{\text {th }}$ percentile error values for the three Chapman parameters. The bottom panel is a histogram of $\xi$ values. Dashed lines mark the $80^{\text {th }}$ and $95^{\text {th }}$ percentile values of these quantities for the a priori ionosphere parameter error vector, except in the bottom panel the dashed line marks the a priori error's mean. 
$\xi$ parameter it should be noted that while its mean value is significantly lower for the base test case, a reduction has also been observed for all 100 runs of Test Case B2's Monte Carlo analysis. Thus, Test Case B2 has the "advantage" of starting with such a poor $a$ priori ionosphere model that it cannot help but deduce an improved model in all 100 of its Monte-Carlo trials.

A posteriori estimation error sensitivity to ground station placement has been studied with Test Case B3. Unlike the grid-like array of ground stations considered with the test cases that have been discussed so far, the setup for Test Case B3 consists of a network of ground stations that are located along the coasts and land borders of the United States. Figure 36 illustrates the setup for this test case. It also shows the simulated true multi-hop ray-paths.

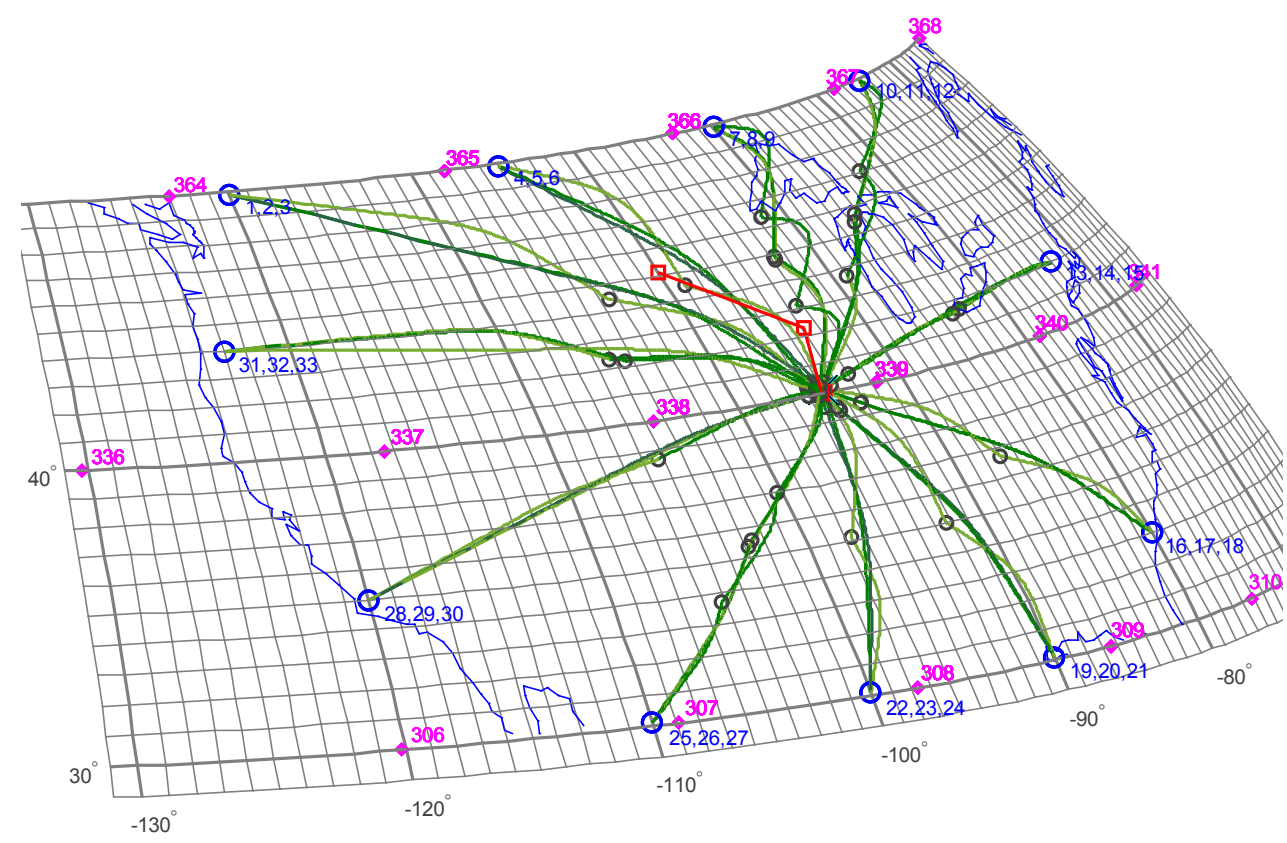

Figure 36: Setup for Test Case B3 with ground stations (blue circles) placed along the coastal and land borders of the US, true ray-paths trajectories (green), and convergence of position solution (red). 
As with previous test cases, each ground station is assumed to transmit three signals that are received at the UE located at latitude/longitude/altitude (LLA) $\left[40.1^{0}\right.$,$\left.95 \cdot 1^{0}, 10000 \mathrm{~m}\right]$. All ionospheric parameters are identical to those of Test Case B0. The different ground station placement results in a different subset of applicable grid nodes and consequently an IEI of -0.2165 that is slightly larger than the value that has been computed for Test Case B0.

A run in Nominal Scenario Mode yielded position errors of -399, 394 and 159 meters in the local north, east, and vertical directions, respectively. The error in the estimate of the receiver clock bias is equivalent to -665 meters. This result is inferior to that of Test Case B0. With the same a priori ionosphere model, the final estimation errors for the electron density altitude parameter $h_{\max }$ (Fig. 37), are similarly worse than those obtained for Test Case B0, with $80 \%$ of CONUS having errors below $5.2 \mathrm{~km}$ and $95 \%$ of CONUS having errors below $6.6 \mathrm{~km}$. Estimates for the $h_{\mathrm{sf}}$ scale height factor and for the VTEC parameter (Fig. 38) exhibit similar degraded levels of accuracy in the region that is common to the two test cases.

Test Case B3 was next executed in Performance Analysis Mode with 100 Monte Carlo runs and a 1-sigma measurement noise of 1000 meters. Results for position errors exhibit an accuracy level that is inferior to that of Test Case B0 in terms of the position of the mean error that is significantly further from zero for Test Case B3 as discussed above. However, this test case's horizontal 90\% error ellipse is smaller (Fig. 39). The lengths of the horizontal ellipse's semi-major and semi-minor axes are approximately 1575 and 

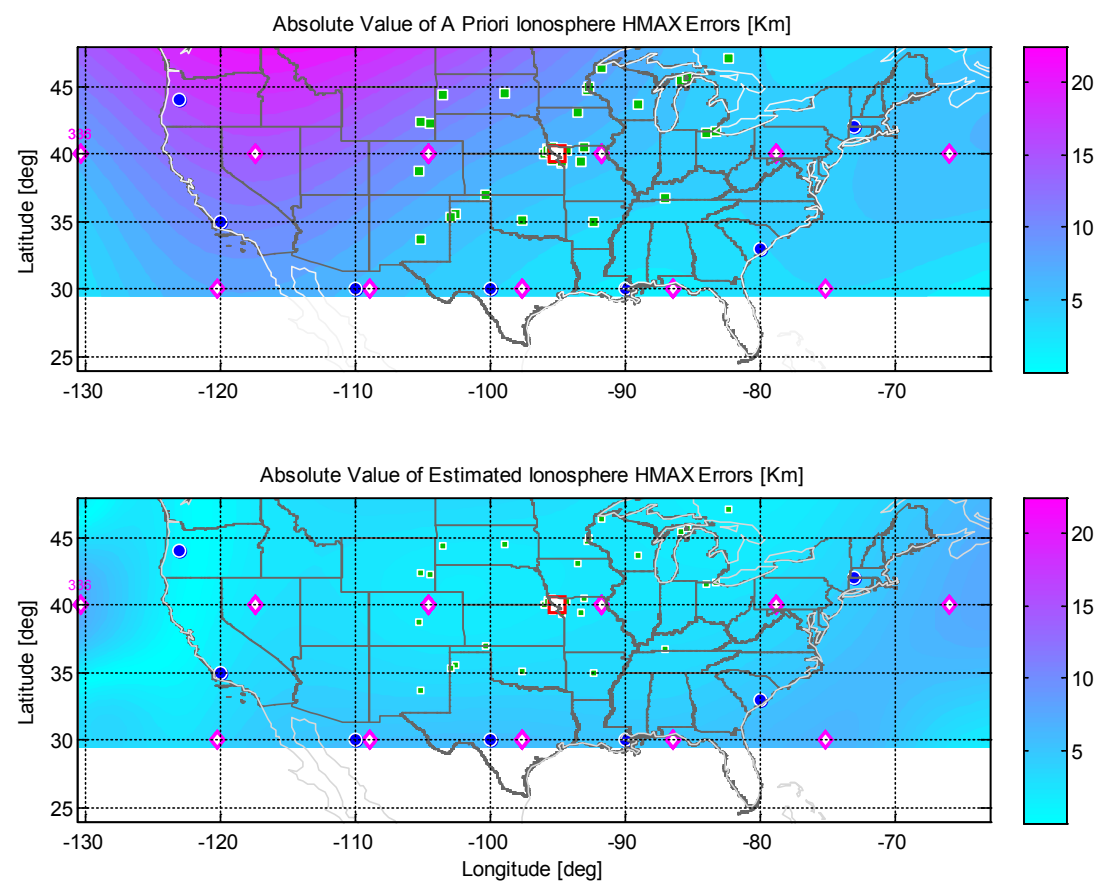

Figure 37: A priori (top) and a posteriori (bottom) errors for the ionospheric peak electron density height parameter $h_{\max }$ for a Nominal Scenario Mode run of Test Case B3.
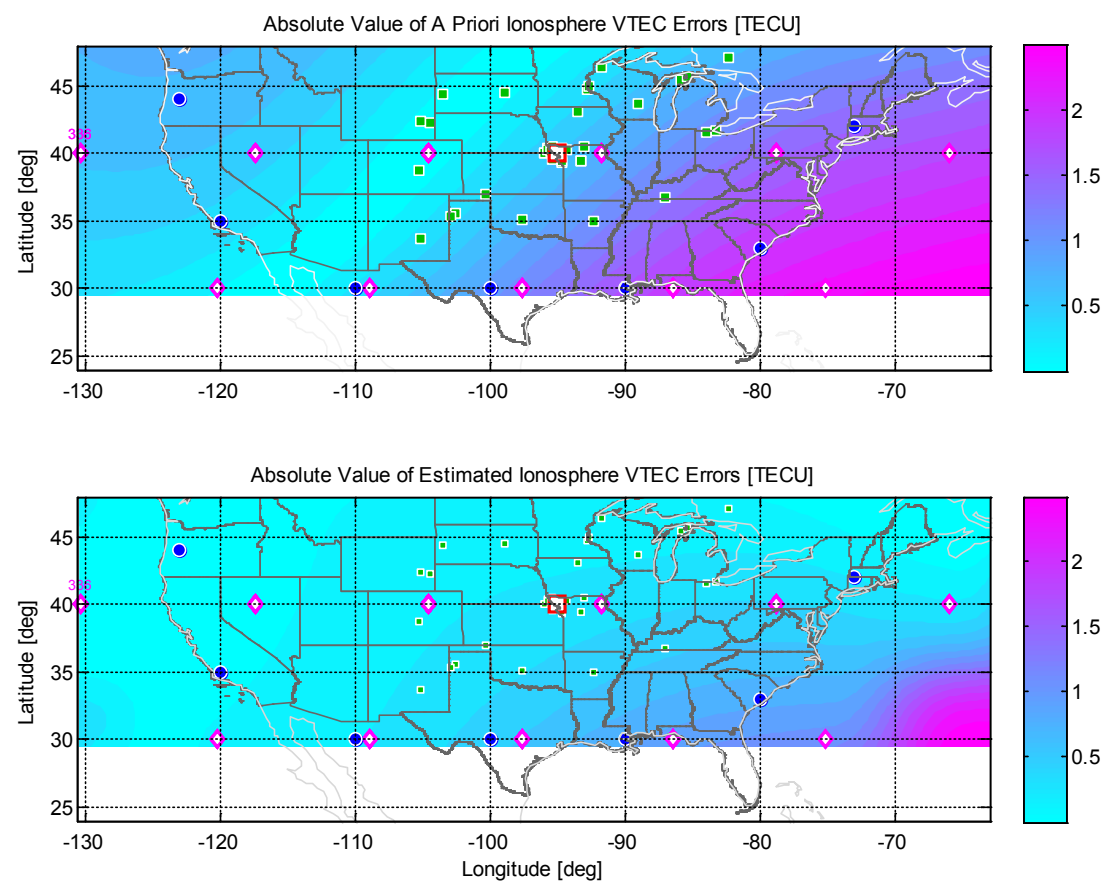

Figure 38: A priori (top) and a posteriori (bottom) errors for the ionospheric vertical total electron content parameter VTEC for a Nominal Scenario Mode run of Test Case B3. 

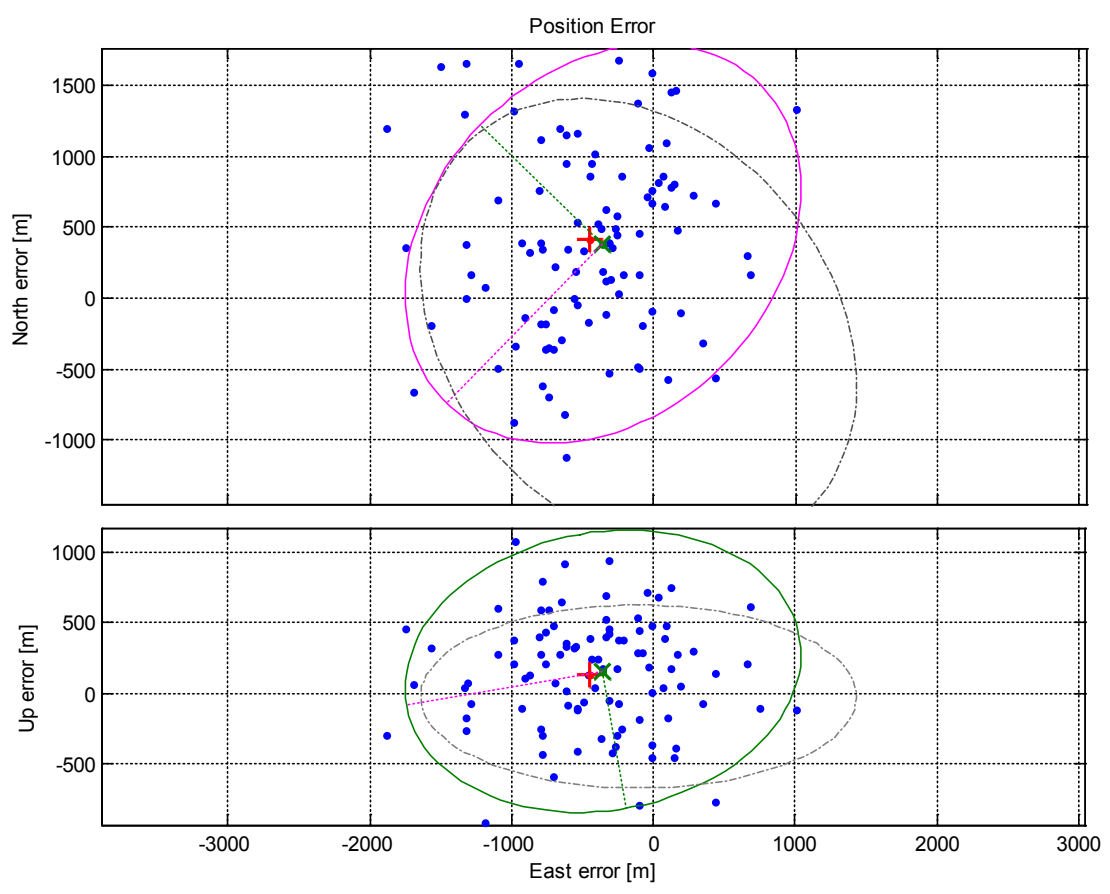

Figure 39: Position error pattern for Test Case B3; Monte Carlo analysis scatter plot (blue); Horizontal 90\% errors ellipse (top plot, magenta); Vertical 90\% errors ellipse (bottom plot, green); The dashed gray ellipses plot the $90 \%$ error ellipses for base Test Case B0 and are shown here for comparison purposes.

1203 meters compared to 1765 and 1347 meters for Test Case B0 ellipse's semi-major and semi-minor axes, respectively. For ionosphere modeling (data not shown), performance for this test case is slightly degraded, especially for estimating the vertical total electron content parameter.

The last variation from the base test case for group B is Test Case B4, for which the measurement noise 1-sigma value is reduced from 1000 meters to 10 meters. This, of course, is an unrealistic value that cannot be achieved based on code ranging solely with current technology. However, this test case is used to examine the performance sensitivity to ranging errors, with the underlying assumption that better technology may 
make it possible to achieve better ranging accuracy, though not necessarily as good as 10 meters.

Position errors for this test case, shown in Fig. 40, reflect dramatically enhanced horizontal-plane accuracy in comparison to that of Test Case B0, as shown in Fig. 28. Still, this reduced amount of error is not quite at a level considered to be navigation grade for many applications. Vertical accuracy, however, is significantly better, with a root mean square value of 6.5 meters. At the same time, a posteriori errors for the ionosphere model's three parameters are roughly $50 \%-70 \%$ of the errors obtained for the base test case.
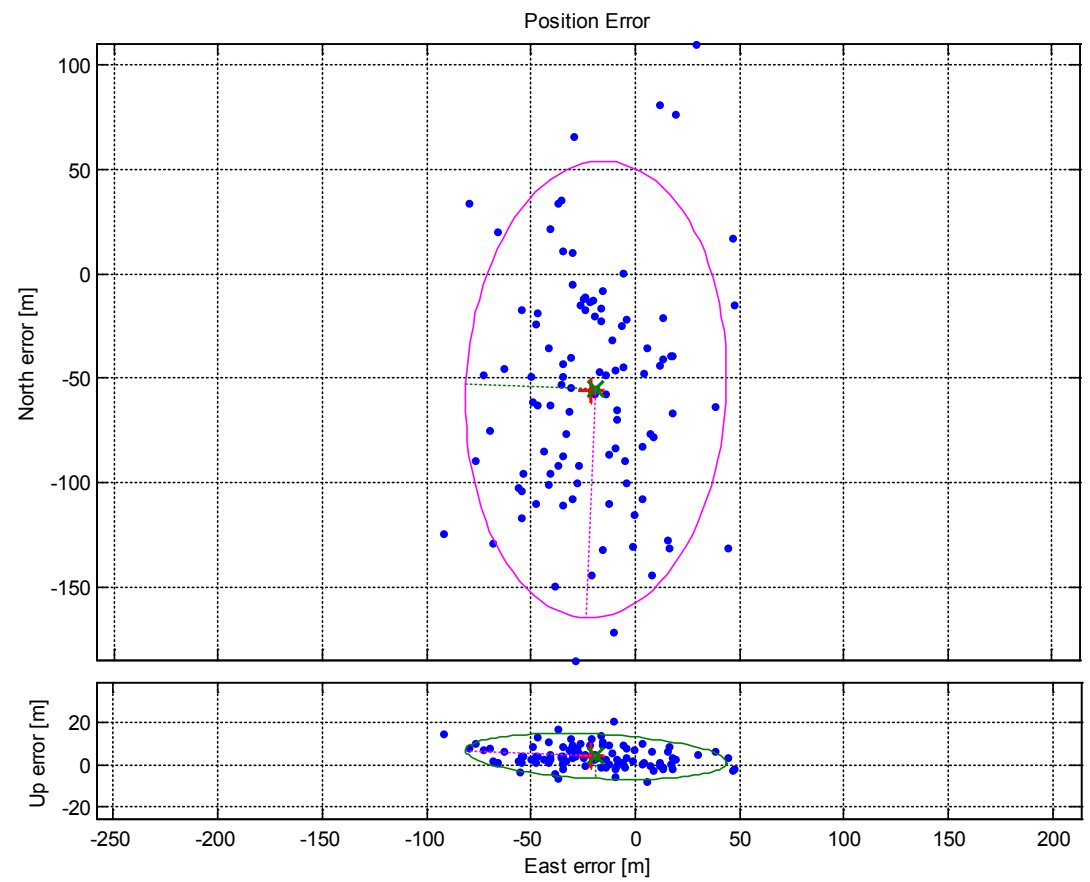

Figure 40: Position error pattern for Test Case B4; Monte Carlo analysis scatter plot (blue); Horizontal 90\% errors ellipse (top plot, magenta); Vertical 90\% errors ellipse (bottom plot, green). 


\subsubsection{Test cases with a low number of available ray-paths (Group A)}

A scenario of limited signal availability is considered in this group of test cases. These test cases are characterized with as few as 21 received signals that are transmitted from 7 ground stations. The set of test cases in this group, therefore, constitutes a study of performance sensitivity to the number of available ray-paths.

The base test case for this group, Test Case A0, has the same properties as Test Case B0, except for the number of available ray-paths. In Nominal Scenario Mode, the total horizontal error for this test case is about $50 \%$ larger than that of Test Case B0, and the vertical error is about twice as large (data not shown). Monte Carlo position errors (Fig. 41) are roughly twice as large as for Test Case B0, both in the horizontal plane and vertically, as is evident by comparison with Test Case B0's ellipses that are also shown in this figure. Errors for the a posteriori ionosphere model in terms of the Chapman parameters' $80^{\text {th }}$ and $95^{\text {th }}$ CONUS area percentiles are consistently bigger for the limited signal availability test case.

It can be concluded that the error computation of Eq. (99), which is based on a linear approximation and from which the $90 \%$ error ellipses are derived, becomes less accurate as fewer measurements are available. In this case, 17 dots fall outside the ellipse when only about 10 should have fallen outside it for the 100-case Monte-Carlo analysis that has produced the results in Fig. 41. This observation is consistent with many tests examined throughout this study, and in particular with the results that have been obtained for Test Case D0 that is discussed later. 


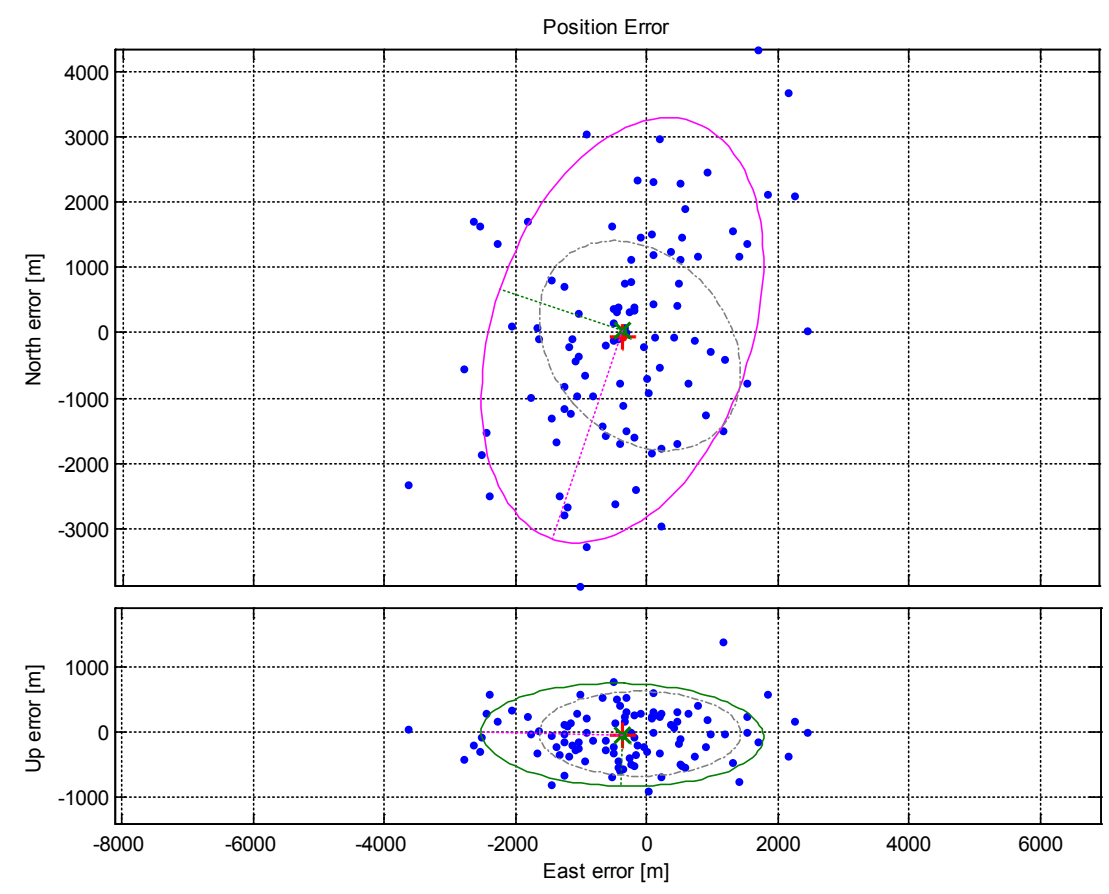

Figure 41: Position error pattern for Test Case A0; Monte Carlo analysis scatter plot (blue); Horizontal 90\% error ellipse (top plot, magenta); Vertical 90\% error ellipse (bottom plot, green). The dashed gray ellipses plot the $90 \%$ error ellipses for base Test Case B0 and are shown here for comparison purposes.

\subsubsection{Test cases with a high number of available ray-paths (Group C)}

The multiple frequencies strategy that has been introduced in Subsection 2.1.1 can be used not only with beat carrier phase measurements as described, but also as a means of increasing the number of available measurements when only code ranging measurements are processed. Test cases in this category differ from test cases of Group B in the number of unique signal frequencies that are used with each ray-path. Unlike with the medium number of available ray-paths test cases, in test cases of Group C each ray-path, characterized by a distinct number of ray-hops, is assumed to be sampled four times as its signal's frequency changes. This means that four measurements are obtained for each combination of transmitter and number of ray-hops. With 32 ray-paths, the total number 
of measurements for these test cases is 128 , as demonstrated in the setup and solution plot of Fig. 42.

Test Case $\mathrm{C} 0$ has the same characteristics as the medium-number-of-availablemeasurements scenario's base test case, Test Case B0, except for the increased number of measurements. Execution in Nominal Scenario Mode yields a mean position error of 104, -74 and -12.36 meters in the north, east, and vertical directions, respectively. This error is about half the magnitude of the position error observed for Test Case B0. It should also be noted that for this test case, actual a posteriori position errors and predicted errors agree to within 0.4 meters, as per Eq. (99). Thus, the linearized error model is a good approximation.

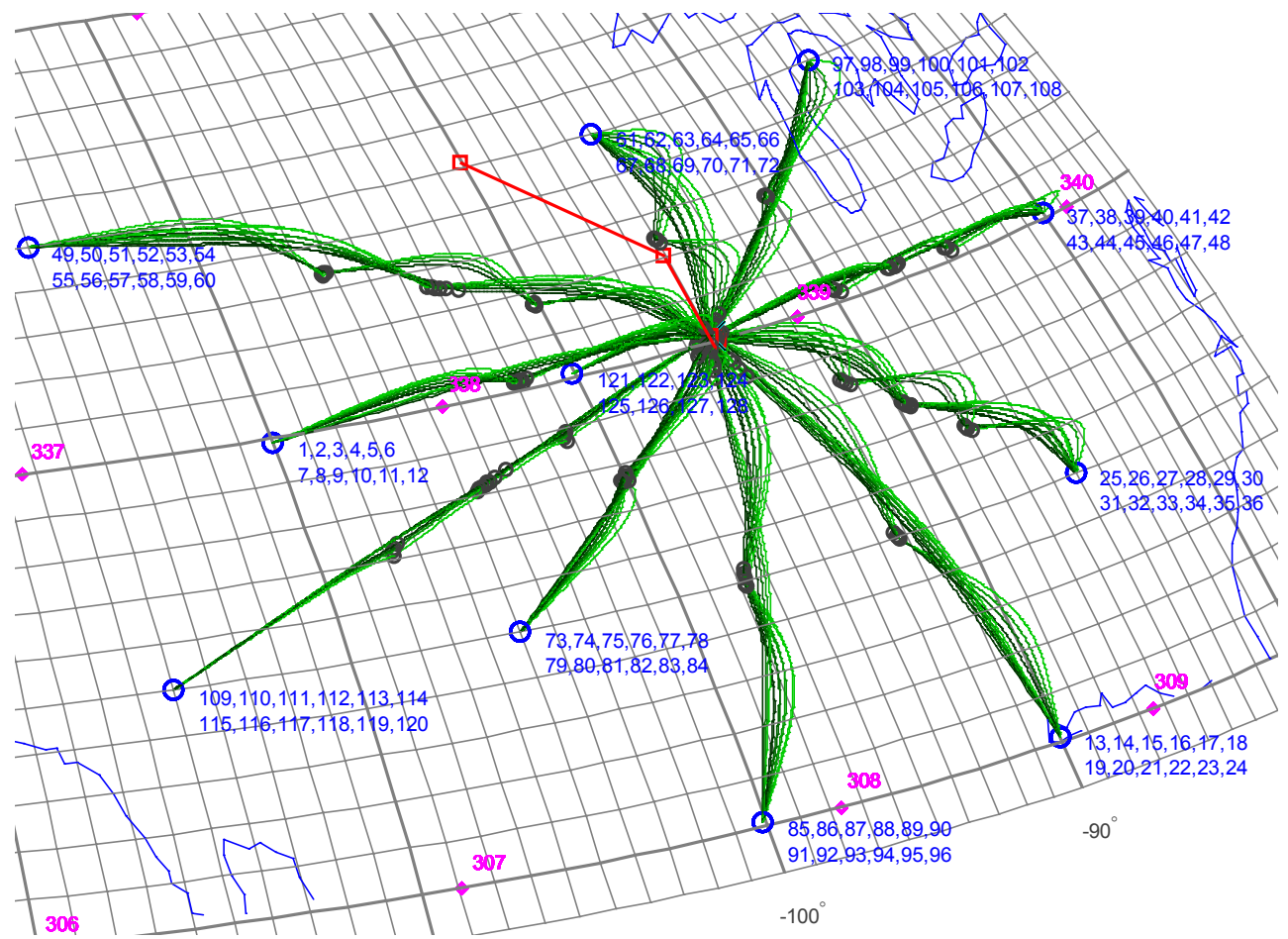

Figure 42: Setup for test cases of Groups $\mathrm{C}$ and E, transmitters (blue circles), true ray-path trajectories (green curves), and convergence of position solution for Test Case C0 (red curve). 
For ionosphere characterization, the nominal execution yielded an $80^{\text {th }}$ percentile of CONUS area upper bound of $3.1 \mathrm{~km}$ for the parameter $h_{\max }$. This value is $20 \%$ smaller than that of Test Case B0. A reduction of $34 \%$ for the $h_{\text {sf }}$ parameter's $80^{\text {th }}$ percentile value has been observed as well. For the vertical total electron content parameter, the $80^{\text {th }}$ percentile's mean value decreased from 0.16 TECU to 0.11 TECU.

The 90\% error ellipses obtained with a Monte Carlo analysis, shown in Fig. 43, are evidently smaller than those of Test Case B0 (also shown in this figure), with semi-major axes that are about half of the value for Test Case B0. Yet, the achieved level of accuracy for Test Case $\mathrm{C} 0$ is insufficient for navigation.

However, with the reduced measurement error standard deviation model that is considered in Test Case C4 (Fig. 44), position errors exhibit accuracy that could be acceptable for navigation in some applications. As earlier noted, the measurement noise level that is considered in this last case is currently not achievable with code ranging only. 

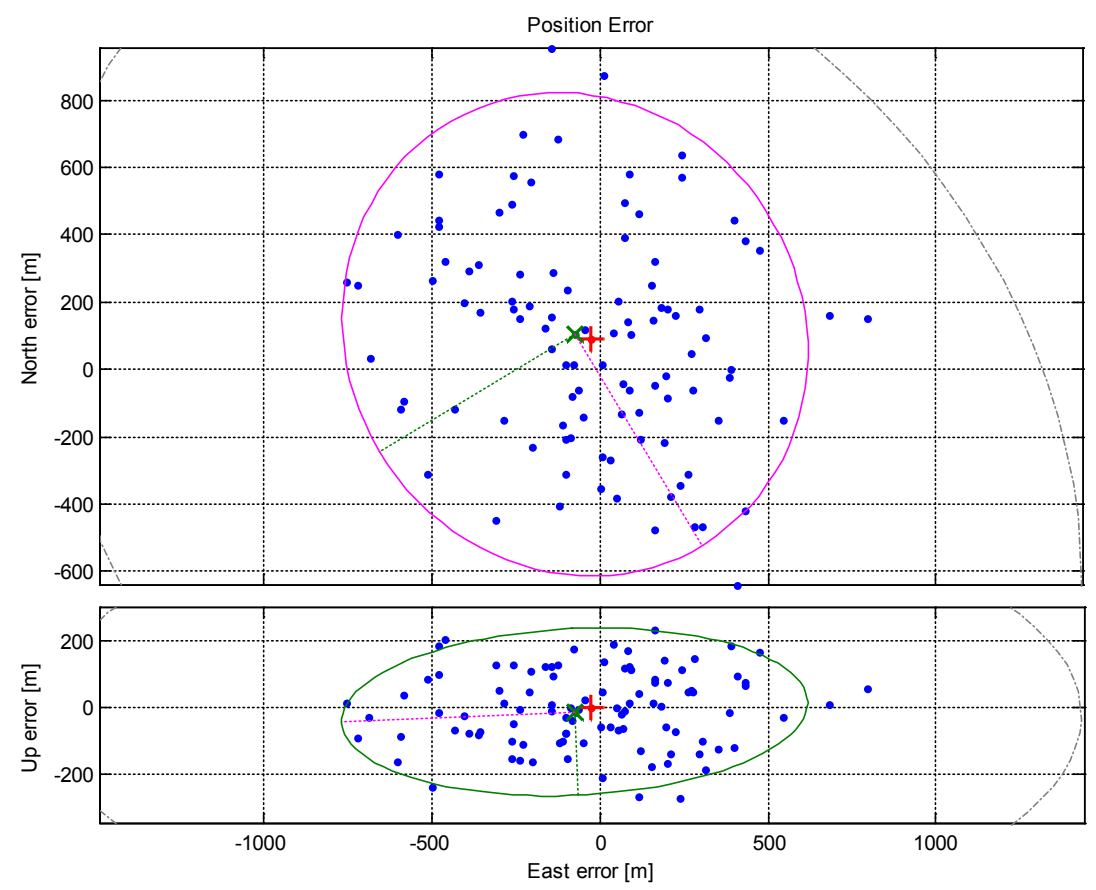

Figure 43: Position error pattern for Test Case C0; Monte Carlo analysis scatter plot (blue); Horizontal 90\% errors ellipse (top plot, magenta); Vertical 90\% errors ellipse (bottom plot, green); The dashed gray ellipses are the $90 \%$ error ellipses for Test Case B0, which are shown here for comparison purposes.
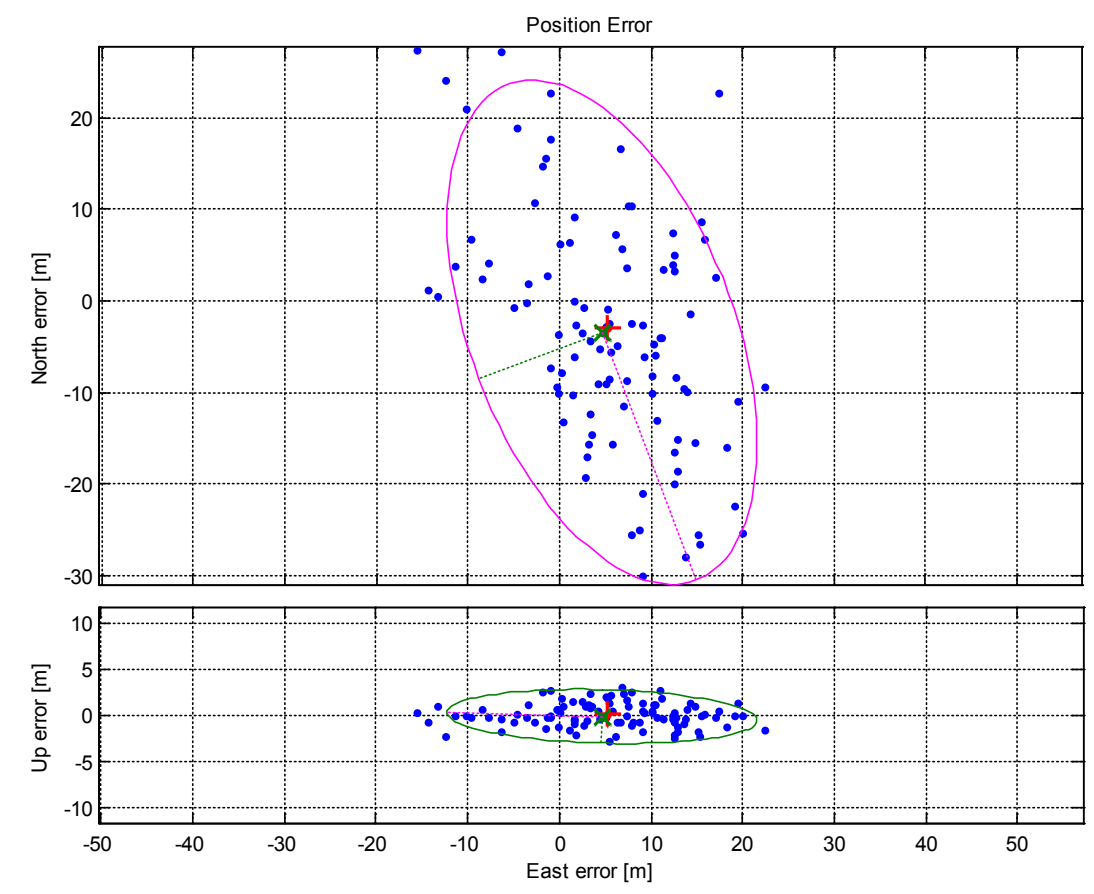

Figure 44: Position error pattern for Test Case C4; Monte Carlo analysis scatter plot (blue); Horizontal $90 \%$ errors ellipse (top plot, magenta); Vertical 90\% errors ellipse (bottom plot, green). 


\subsubsection{Test cases using only group delay, summary}

Table 2 summarizes the numerical results for the eight test cases presented in this section in terms of position accuracy. The location of the computed mean error is given in a local level north-east-vertical coordinates system whose origin is located at the true location of the receiver. Horizontal position errors are expressed in term of a horizontal error ellipse that bounds $90 \%$ of the computed east-north errors. The vertical error is given by its standard deviation.

\begin{tabular}{|c|c|c|c|c|c|c|}
\hline & & base case & $\begin{array}{l}\text { ray-paths } \\
\text { from } \\
\text { above only }\end{array}$ & $\begin{array}{c}\text { large } \\
\text { initial } \xi\end{array}$ & $\begin{array}{c}\text { alternative } \\
\text { stations } \\
\text { placement }\end{array}$ & $\begin{array}{c}\text { reduced } \\
\text { meas. } \\
\text { noise }\end{array}$ \\
\hline \multirow{5}{*}{$\begin{array}{c}\text { Low } \\
\text { number of } \\
\text { available } \\
\text { signals } \\
\text { Scenario } \\
\text { (Group A) }\end{array}$} & test case \# & $A 0$ & & & & \\
\hline & $\begin{array}{l}90 \% \text { error ellipse } \\
\text { semi-major axis }[\mathrm{m}]\end{array}$ & 3359 & & & & \\
\hline & $\begin{array}{l}90 \% \text { error ellipse } \\
\text { semi-minor axis [m] }\end{array}$ & 1972 & & & & \\
\hline & $\begin{array}{l}\text { vertical error STD } \\
{[\mathrm{m}]}\end{array}$ & 385 & & & & \\
\hline & $\begin{array}{l}\text { mean error NEV } \\
{[\mathrm{m}, \mathrm{m}, \mathrm{m}]}\end{array}$ & $\begin{array}{c}-13,-367 \\
-45\end{array}$ & & & & \\
\hline \multirow{5}{*}{$\begin{array}{l}\text { Medium } \\
\text { number of } \\
\text { available } \\
\text { signals } \\
\text { Scenario } \\
\text { (Group B) }\end{array}$} & test case \# & BO & $B 1$ & $B 2$ & $B 3$ & B4 \\
\hline & $\begin{array}{l}90 \% \text { error ellipse } \\
\text { semi-major axis }[\mathrm{m}]\end{array}$ & 1765 & 1973 & 1817 & 1575 & 110 \\
\hline & $\begin{array}{l}90 \% \text { error ellipse } \\
\text { semi-minor axis }[\mathrm{m}]\end{array}$ & 1347 & 1249 & 1330 & 1203 & 62 \\
\hline & $\begin{array}{l}\text { vertical error STD } \\
{[\mathrm{m}]}\end{array}$ & 311 & 1885 & 309 & 435 & 5 \\
\hline & $\begin{array}{l}\text { mean error NEV } \\
{[\mathrm{m}, \mathrm{m}, \mathrm{m}]}\end{array}$ & $\begin{array}{c}-197,-104 \\
-24\end{array}$ & $\begin{array}{c}218,-180 \\
76\end{array}$ & $\begin{array}{c}597,-269 \\
-50\end{array}$ & $\begin{array}{l}394,-399 \\
158\end{array}$ & $\begin{array}{c}-55,-19 \\
4\end{array}$ \\
\hline \multirow{5}{*}{$\begin{array}{c}\text { High } \\
\text { number of } \\
\text { available } \\
\text { signals } \\
\text { Scenario } \\
\text { (Group C) }\end{array}$} & test case \# & CO & & & & C4 \\
\hline & $\begin{array}{l}90 \% \text { error ellipse } \\
\text { semi-major axis }[\mathrm{m}]\end{array}$ & 733 & & & & 29 \\
\hline & $\begin{array}{l}90 \% \text { error ellipse } \\
\text { semi-minor axis }[\mathrm{m}]\end{array}$ & 677 & & & & 14 \\
\hline & $\begin{array}{l}\text { vertical error STD } \\
{[\mathrm{m}]}\end{array}$ & 116 & & & & 10 \\
\hline & $\begin{array}{l}\text { mean error NEV } \\
{[\mathrm{m}, \mathrm{m}, \mathrm{m}]}\end{array}$ & $\begin{array}{c}103,-74 \\
-12\end{array}$ & & & & $-3,5,0$ \\
\hline
\end{tabular}

Table 2: Class 1 test cases' position estimation error characteristics. 
A summary of results for ionosphere model errors is presented in Table 3. The first three rows for each category consist of the a priori (upper pair of cells) and the mean of the a posteriori (lower pair of cells) estimation errors for the three Chapman parameters. For each pair of values, the left refers to the $80^{\text {th }}$ CONUS area percentile value and the right to the $95^{\text {th }}$ percentile value. The fourth row shows the a priori and the mean $a$ posteriori IEI values that have been computed for each test case.

\begin{tabular}{|c|c|c|c|c|c|c|c|}
\hline & & & $\begin{array}{l}\text { base } \\
\text { case }\end{array}$ & $\begin{array}{l}\text { ray-paths } \\
\text { from } \\
\text { above only }\end{array}$ & $\begin{array}{c}\text { large } \\
\text { initial } \xi\end{array}$ & $\begin{array}{c}\text { alternative } \\
\text { stations } \\
\text { placement }\end{array}$ & $\begin{array}{c}\text { reduced } \\
\text { meas. } \\
\text { noise }\end{array}$ \\
\hline \multirow{5}{*}{$\begin{array}{c}\text { Low } \\
\text { number of } \\
\text { available } \\
\text { signals } \\
\text { Scenario } \\
\text { (Group A) }\end{array}$} & test case \# & & AO & & & & \\
\hline & $\begin{array}{l}h_{\max } 80^{\text {th }} / 95^{\text {th }} \\
\text { percentile }[\mathrm{km}]\end{array}$ & $\begin{array}{c}\text { a priori } \\
\text { mean } \text { a post. }\end{array}$ & $\begin{array}{c}14.1 / 20.2 \\
1.6 / 2.6\end{array}$ & & & & \\
\hline & $\begin{array}{l}h_{\mathrm{sf}} 80^{\text {th }} / 95^{\text {th }} \\
\text { percentile }[\mathrm{km}]\end{array}$ & $\begin{array}{c}\text { a priori } \\
\text { mean } \text { a post. }\end{array}$ & $\begin{array}{l}6.7 / 7.7 \\
1.1 / 2.4\end{array}$ & & & & \\
\hline & $\begin{array}{l}V T E C 80^{\text {th }} / 95^{\text {th }} \\
\text { percentile }[\text { TECU] }\end{array}$ & $\begin{array}{c}\text { a priori } \\
\text { mean } \text { a post. }\end{array}$ & $\begin{array}{c}1.2 / 1.9 \\
0.14 / 0.22\end{array}$ & & & & \\
\hline & $\xi$ & $\begin{array}{c}\text { a priori } \\
\text { mean } \text { a post. }\end{array}$ & $\begin{array}{l}-0.2276 \\
-0.2355\end{array}$ & & & & \\
\hline \multirow{5}{*}{$\begin{array}{l}\text { Medium } \\
\text { number of } \\
\text { available } \\
\text { signals } \\
\text { Scenario } \\
\text { (Group B) }\end{array}$} & test case \# & & BO & B1 & $B 2$ & B3 & B4 \\
\hline & $\begin{array}{l}h_{\max } 80^{\text {th }} / 95^{\text {th }} \\
\text { percentile }[\mathrm{km}]\end{array}$ & $\begin{array}{c}\text { a priori } \\
\text { mean } \text { a post. }\end{array}$ & $\begin{array}{l}14.1 / 20.2 \\
2.1 / 3.6\end{array}$ & $\begin{array}{c}14.1 / 20.2 \\
1.5 / 4.1\end{array}$ & $\begin{array}{l}27.2 / 46.3 \\
10.3 / 17.4\end{array}$ & $\begin{array}{l}13.1 / 19.4 \\
5.2 / 6.6\end{array}$ & $\begin{array}{l}14.1 / 20.2 \\
4.5 / 8.5\end{array}$ \\
\hline & $\begin{array}{l}h_{\mathrm{sf}} 80^{\text {th }} / 95^{\text {th }} \\
\text { percentile }[\mathrm{km}]\end{array}$ & $\begin{array}{c}\text { a priori } \\
\text { mean } \text { a post. }\end{array}$ & $\begin{array}{l}6.7 / 7.7 \\
1.2 / 2.8\end{array}$ & $\begin{array}{l}6.7 / 7.7 \\
1.2 / 2.7\end{array}$ & $\begin{array}{c}19.8 / 21.1 \\
2.9 / 3.5\end{array}$ & $\begin{array}{l}7.6 / 8.2 \\
2.3 / 2.8\end{array}$ & $\begin{array}{l}6.7 / 7.7 \\
1.8 / 3.1\end{array}$ \\
\hline & $\begin{array}{l}V T E C 80^{\text {th }} / 95^{\text {th }} \\
\text { percentile }[\text { TECU }]\end{array}$ & $\begin{array}{c}\text { a priori } \\
\text { mean } \text { a post. }\end{array}$ & $\begin{array}{l}1.2 / 1.9 \\
0.16 / 0.21\end{array}$ & $\begin{array}{l}1.2 / 1.9 \\
0.12 / 0.21\end{array}$ & $\begin{array}{l}1.7 / 2.7 \\
1.0 / 1.4\end{array}$ & $\begin{array}{l}1.6 / 2.2 \\
0.55 / 1.1\end{array}$ & $\begin{array}{c}1.2 / 1.9 \\
0.38 / 0.61\end{array}$ \\
\hline & $\xi$ & $\begin{array}{c}\text { a priori } \\
\text { mean } \text { a post. }\end{array}$ & $\begin{array}{l}-0.2276 \\
-0.2375\end{array}$ & $\begin{array}{l}-0.2276 \\
-0.2399\end{array}$ & $\begin{array}{l}-0.0661 \\
-0.0955\end{array}$ & $\begin{array}{l}-0.2165 \\
-0.2224\end{array}$ & $\begin{array}{l}-0.2276 \\
-0.2509\end{array}$ \\
\hline \multirow{5}{*}{$\begin{array}{c}\text { High } \\
\text { number of } \\
\text { available } \\
\text { signals } \\
\text { Scenario } \\
\text { (Group C) }\end{array}$} & test case \# & & CO & & & & $C 4$ \\
\hline & $\begin{array}{l}h_{\max } 80^{\text {th }} / 95^{\text {th }} \\
\text { percentile }[\mathrm{km}]\end{array}$ & $\begin{array}{c}\text { a priori } \\
\text { mean } \text { a post. }\end{array}$ & $\begin{array}{c}14.1 / 20.2 \\
1.3 / 3.0\end{array}$ & & & & $\begin{array}{l}14.1 / 20.2 \\
0.34 / 0.76\end{array}$ \\
\hline & $\begin{array}{l}h_{\mathrm{sf}} 80^{\text {th }} / 95^{\text {th }} \\
\text { percentile }[\mathrm{km}]\end{array}$ & $\begin{array}{c}\text { a priori } \\
\text { mean } \text { a post. }\end{array}$ & $\begin{array}{c}6.7 / 7.7 \\
0.87 / 1.9\end{array}$ & & & & $\begin{array}{c}6.7 / 6.6 \\
0.26 / 0.73\end{array}$ \\
\hline & $\begin{array}{l}V T E C 80^{\text {th }} / 95^{\text {th }} \\
\text { percentile }[\text { TECU] }\end{array}$ & $\begin{array}{c}\text { a priori } \\
\text { mean } \text { a post. }\end{array}$ & $\begin{array}{c}1.2 / 1.9 \\
0.11 / 0.20\end{array}$ & & & & $\begin{array}{l}1.2 / 1.9 \\
0.03 / 0.08\end{array}$ \\
\hline & $\xi$ & $\begin{array}{c}\text { a priori } \\
\text { mean a post. }\end{array}$ & $\begin{array}{l}-0.2276 \\
-0.2471\end{array}$ & & & & $\begin{array}{l}-0.2276 \\
-0.2915\end{array}$ \\
\hline
\end{tabular}

Table 3: Class 1 test cases' ionosphere model estimation error characteristics. 


\subsection{Test Cases Using Combined Code and Carrier Phase Ranging, Fixed Ionosphere Model (Class 2)}

The purpose of this section is to study the performance of the system for the combined group-delay/beat-carrier-phase batch filter. With the addition of carrier phase measurements, the truth-model simulation must receive as input the initial errors for all parameters that have been listed for the case of the group-delay-only filter. In addition, it must be given truth values of the carrier phase biases for each ray path. The same bias is used for each of the four frequencies that are transmitted in succession along a given nominal ray path. These extra unknowns are expected to be observable because of the availability of beat carrier phase measurements at multiple frequencies that share a common bias. It should be additionally noted that no a priori information is assumed for these highly observable terms. Each batch filter run has been initialized with zero values for the carrier phase bias estimates. In order to ensure that this part of the analysis is not carried out with unrealistically small errors for the zero-valued a priori estimates of the bias terms, the truth-model simulation has been run with bias values that produced initial range-equivalent errors for these terms up to $1600 \mathrm{~km}$.

Table 4 summarizes the primary characteristics for all seven Class 2 test cases analyzed in this section (Test Cases D0, D2, and E0-E4): their standard deviation for both group delay and beat carrier phase measurement noise, number of ray-paths, initial IEI, direction of signals as they arrive at the receiver, and the placement pattern for the ground stations from which received signals were broadcast. Note that the measurement noise lists the range-equivalent group delay measurement error standard deviation first, followed by the range-equivalent beat carrier phase measurement error standard delay 
second. Thus, the entry 1000/1 [m] indicates a 1000 meters range-equivalent group delay measurement error standard deviation and a 1 meter range-equivalent beat carrier phase measurement error standard deviation.

\begin{tabular}{|c|c|c|c|c|c|c|}
\hline & & $\begin{array}{l}\text { base } \\
\text { case }\end{array}$ & $\begin{array}{l}\text { ray-paths } \\
\text { from } \\
\text { above only }\end{array}$ & $\begin{array}{c}\text { large } \\
\text { initial } \xi\end{array}$ & $\begin{array}{c}\text { alternative } \\
\text { stations } \\
\text { placement }\end{array}$ & $\begin{array}{l}\text { increased } \\
\text { meas. noise }\end{array}$ \\
\hline \multirow{6}{*}{$\begin{array}{c}\text { Medium } \\
\text { number of } \\
\text { available } \\
\text { signals } \\
\text { Scenario } \\
\text { (Group D) }\end{array}$} & test case \# & DO & & D2 & & \\
\hline & meas. noise $\sigma[\mathrm{m}]$ & $1000 / 1$ & & $1000 / 1$ & & \\
\hline & ray-paths & $17 \times 4$ & & $17 \times 4$ & & \\
\hline & $\xi$ & -0.2276 & & 0.0848 & & \\
\hline & $\begin{array}{l}\text { wave arrival } \\
\text { direction }\end{array}$ & both & & both & & \\
\hline & stations placement & grid & & grid & & \\
\hline \multirow{6}{*}{$\begin{array}{c}\text { High } \\
\text { number of } \\
\text { available } \\
\text { signals } \\
\text { Scenario } \\
\text { (Group E) }\end{array}$} & test case \# & EO & E1 & $E 2$ & E3 & E4 \\
\hline & meas. noise $\sigma[\mathrm{m}]$ & $1000 / 1$ & $1000 / 1$ & $1000 / 1$ & $1000 / 1$ & $1000 / 10$ \\
\hline & ray-paths & $32 \times 4$ & $32 \times 4$ & $32 \times 4$ & $32 \times 4$ & $32 \times 4$ \\
\hline & $\xi$ & -0.2276 & -0.2276 & 0.0661 & -0.2165 & -0.2276 \\
\hline & $\begin{array}{l}\text { wave arrival } \\
\text { direction }\end{array}$ & both & above only & both & both & both \\
\hline & stations placement & grid & grid & grid & perimeter & grid \\
\hline
\end{tabular}

Table 4: Setup configuration for two groups and seven test cases of Class 2.

The two groups of test cases cover scenarios of medium and high numbers of available measurements. As before, for each group (or availability scenario), a base test case is evaluated first. These are Test Cases D0 and E0 for groups D and E, respectively. As with Class 1 test cases, sensitivities to the setup's parameters are examined through examining variations from the base test cases. In order to maximize comparability, all test cases have the same true parameterized ionosphere model, which is identical to the model used with Class 1 test cases.

Measurement errors were generated using a Gaussian, zero mean distribution with a 1sigma value of 1000 meters for group delay measurements and 1 meter for carrier phase measurements (except for the increased measurement noise test cases). 


\subsubsection{Test cases with a high number of available ray-paths (Group E)}

Test cases of Group E consider an array of eleven ground station transmitters at various locations across the Continental United States (CONUS). This is the same setup used with Group C's test cases that is illustrated in Fig. 42. The total number of ray-paths available is $32 \times 4=128$, except this time carrier phase measurements are processed as well, resulting in a total of 256 available observables.

Test Case E0 is regarded as the base test case for the high number of ray-paths scenario. All other test cases in Group E are variants on this test case that are used to assess performance sensitivity to inputs. The truth ionosphere electron density profile is based on the IRI model computed for October 23, 2009, at UTC 14:22. The a priori model is based on the model computed for September 23, 2009, at the same hour, such that the total seasonal discrepancy is one month, and the corresponding Ionosphere Error Index $(\xi)$ is -0.2276 , as with most test cases that have been discussed so far. The HF signals for this test case have frequencies in the range 3.0-6.0 MHz. The number of hops for each ray-path is $1-4$, with a mixture of signals arriving from above the UE and signals arriving at the UE from below.

Figure 45 plots position errors obtained with a 100-run Monte Carlo analysis. The benefits of processing the beat carrier phase measurements are immediately evident when the results shown in this plot are compared with the results obtained for the 128 ray-paths Test Case C0. The mean position error for Test Case E0 is as small as 5 meters in the north direction and less than $30 \mathrm{~cm}$ in both the east and vertical directions. The lengths of the $90 \%$ error ellipse's semi-major and semi-minor axes are 24 and 13 meters, 
respectively. The standard deviation for the vertical error is 0.8 meters. Residual estimation errors for the three Chapman parameters are remarkably small for all 100 runs of the Monte Carlo analysis, as shown in Fig. 46.

Looking at the mean residual errors for the parameter $h_{\mathrm{sf}}$, as obtained using the Nominal Scenario Mode execution of the simulation, (Fig. 47) and the histogram results of Fig. 46, one can conclude that the combination of high measurements availability and small measurement noise for the carrier phase measurements results in substantially enhanced estimates for the three Chapman parameters above much of CONUS.

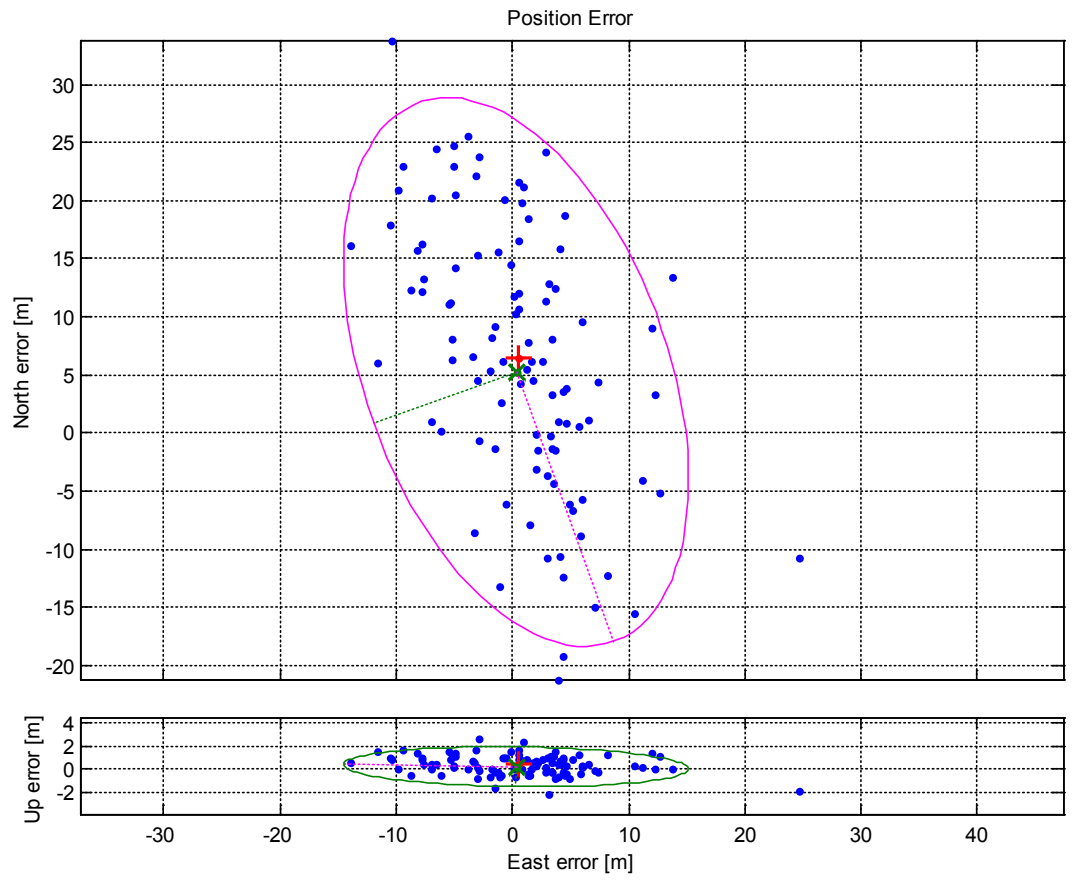

Figure 45: Position error pattern for Test Case E0; Monte Carlo analysis scatter plot (blue); Horizontal 90\% errors ellipse (top plot, magenta); Vertical 90\% errors ellipse (bottom plot, green). 

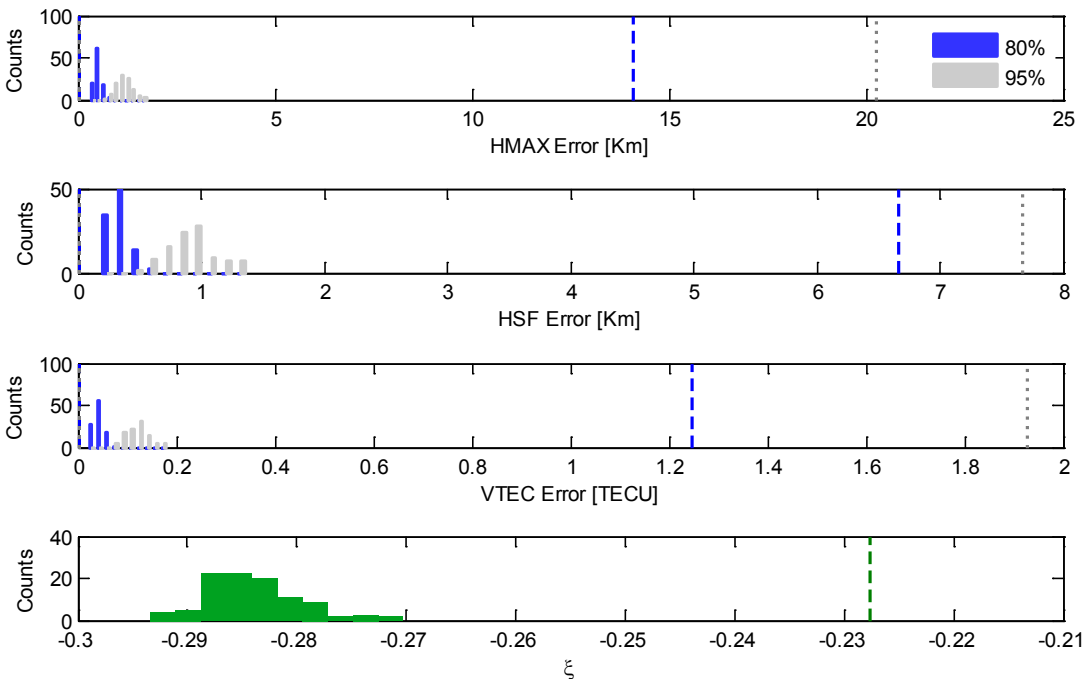

Figure 46: The statistical characteristics of the a posteriori ionosphere model errors for Test Case E0. The top three panels contain histograms of the $80^{\text {th }}$ and $95^{\text {th }}$ CONUS area percentile error values for the three Chapman parameters. The bottom panel is a histogram of $\xi$ values. Dashed lines mark the $80^{\text {th }}$ and $95^{\text {th }}$ percentile values of these quantities for the $a$ priori ionosphere parameter error vector, except in the bottom panel the dashed line marks the a priori error's mean.
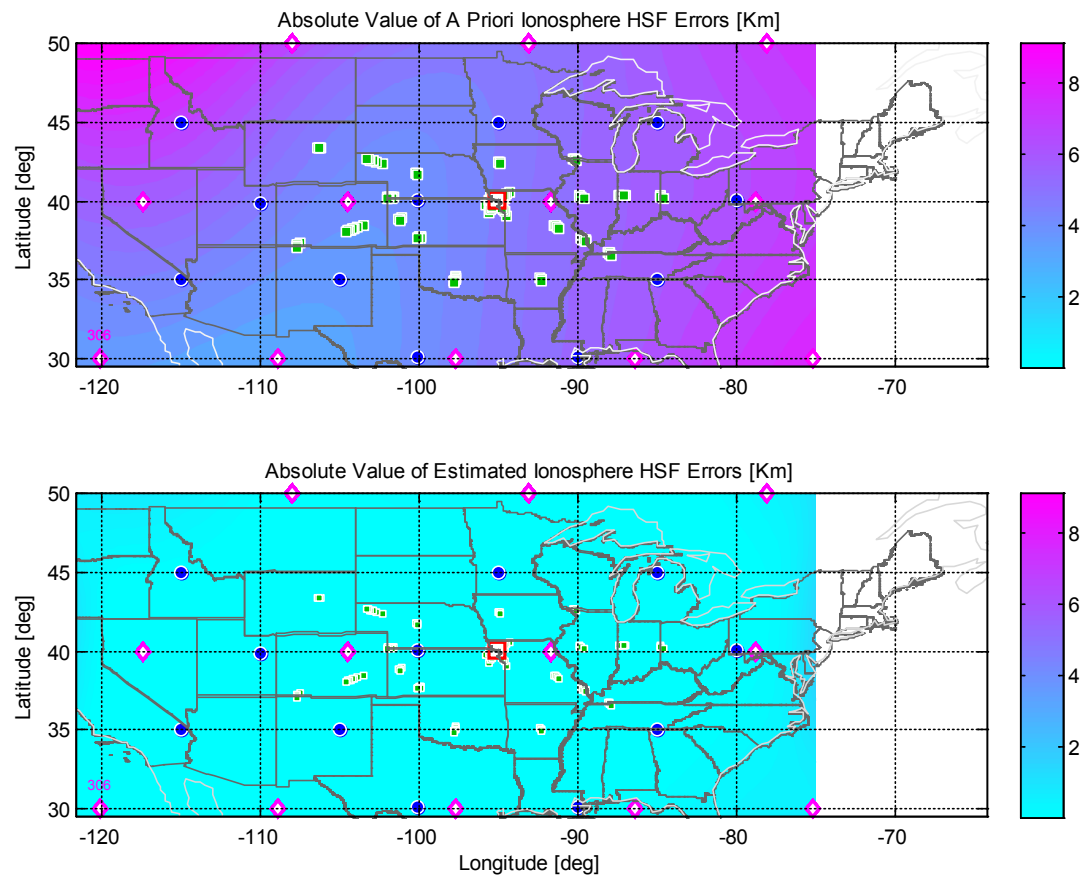

Figure 47: A priori (top) and a posteriori (bottom) errors for the ionospheric peak electron density height $h_{\text {sf }}$ parameter for Test Case E0. 
Test Case E0 has 32 ray-paths, each produced data at four different carrier frequencies. These new measurements added 32 unknown beat carrier phase bias terms. With the Monte Carlo simulation, these terms were set such that their initial errors (which equal their true values given zero a priori guesses) were in the range $[-240,70]$ in units of wavelengths. Given the signals' frequencies, this range of values is equivalent to ranging errors that are up to 1600 kilometers in magnitude. It has been observed that in the first Gauss Newton iteration where carrier phase measurements were processed, these a priori errors are reduced to errors in the range of $[-0.16,0.1]$ wavelengths. Their a posteriori estimates fall in the range $[-0.1,0.1]$. These final error values are equivalent to $5-10$ meter-level ranging errors.

The first variant from the base test case is Test Case E1. It is defined with ray-paths that arrive at the receiver from above. Position error performance in terms of horizontal errors is evidently superior for this test case. It is characterized by a significantly smaller $90 \%$ errors ellipse and a mean error that is closer to zero (Fig. 48). The inferior vertical accuracy, where the standard deviation is 5.2 meters, is likely acceptable for navigation purposes in many applications.

Statistics for the a posteriori Chapman parameters' estimates are similar to those of the base test case, with slightly smaller residual errors for the case of signals arriving from above only. 

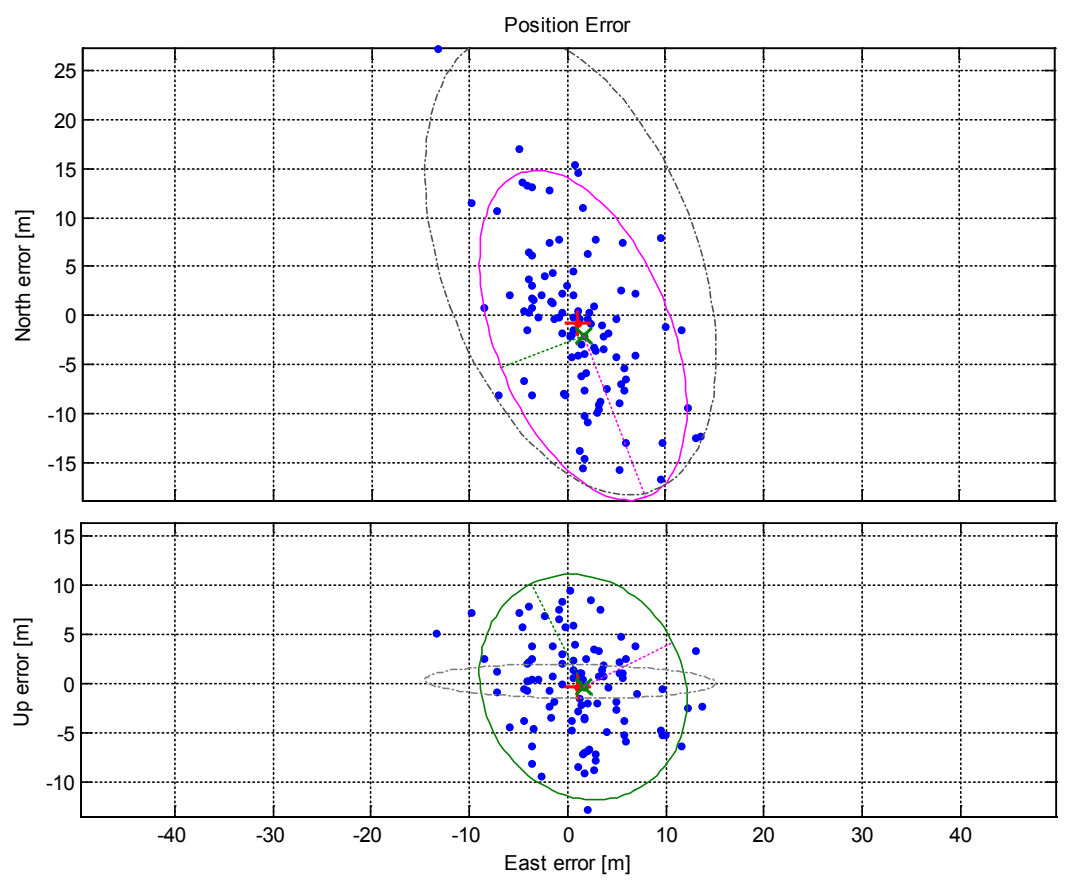

Figure 48: Position error pattern for Test Case E1; Monte Carlo analysis scatter plot (blue); Horizontal 90\% errors ellipse (top plot, magenta); Vertical 90\% errors ellipse (bottom plot, green); The dashed gray ellipses are the $90 \%$ errors ellipses for base Test Case E0, shown here for purposes of comparison.

Test Case E2, a second variant of the base test case, is of special interest due to its very poor a priori ionosphere model that is based on an IRI model computed for August 23,2009 , at UTC 14:22. The resulting a priori IEI value of 0.0848 suggests that this test case is characterized by the largest truth/a priori ionosphere models discrepancy among all test cases studied. Moreover, the IRI $(\xi)$ distribution shown in Fig. 2 suggests that this level of IRI gives this test case's setup an excessive discrepancy between the truth and $a$ priori ionospheres, even though that figure has been generated under slightly different conditions. A priori errors for the VTEC Chapman parameter, shown in the top plot of Fig. 49 , are about $50 \%$ percent larger than those of the base test case. A similar difference of $50 \%$ has been deduced for the parameter $h_{\max }$, while for the parameter $h_{\mathrm{sf}}$ the 
difference is $150 \%$ (data not shown). All of the foregoing a priori parameter errors are measured in terms of the $80^{\text {th }}$ CONUS area percentile.
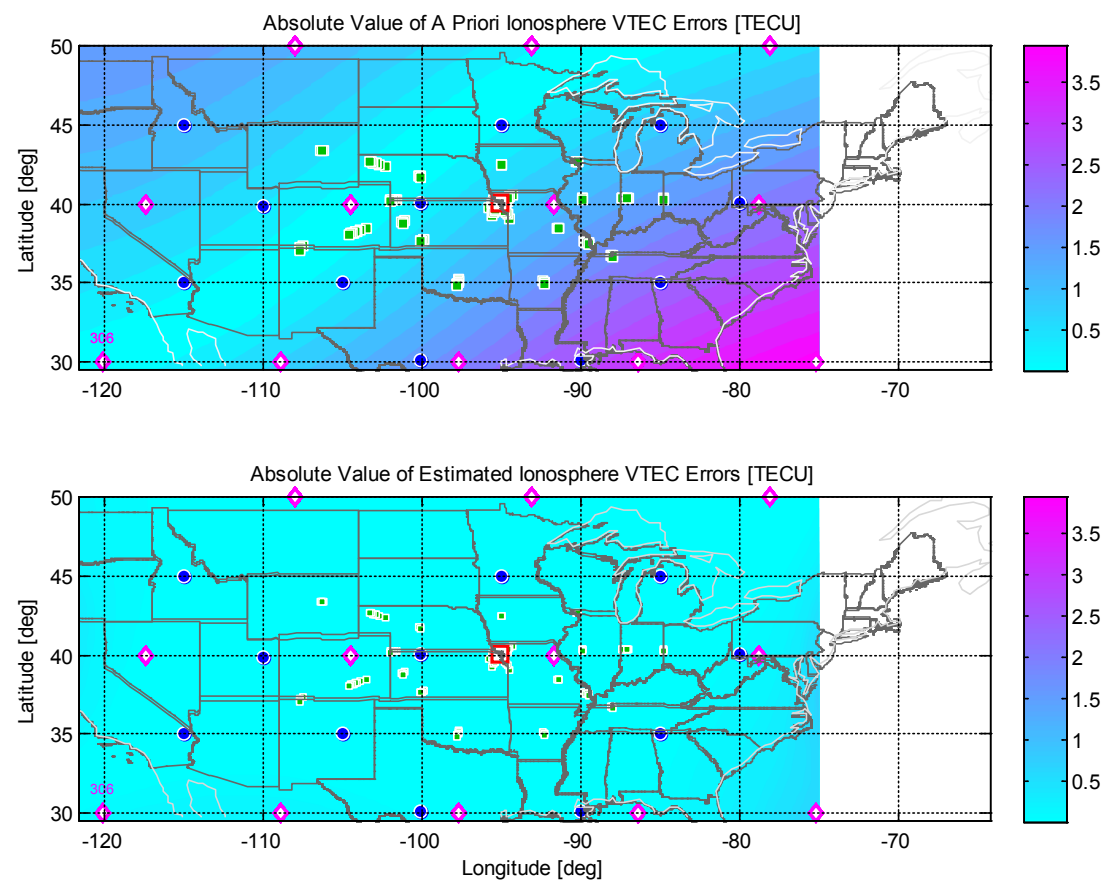

Figure 49: A priori (top) and a posteriori (bottom) errors for the ionospheric vertical total electron content parameter VTEC for a Nominal Scenario Mode run of Test Case E2.

Monte Carlo analysis for this test case yielded the position error scatter plot of Fig. 50, which has two important properties. The area of the $90 \%$ error ellipse is about the same as that of Test Case E0's ellipse, but the mean error, which is the error caused by the ionosphere, is located about three times further from zero. The latter outcome could be predicted based on Eq. (99).

The Monte Carlo analysis yields statistics for the a posteriori errors of the three Chapman parameters. It can be concluded that the errors are about twice as large for Test Case E2 as for the base test case, with a mean error of $0.91 \mathrm{~km}$ for the parameter $h_{\max }$, a 
mean error of $0.46 \mathrm{~km}$ for the parameter $h_{s f}$, and a mean error of 0.12 TECU for the parameter VTEC, all given in terms of their $80^{\text {th }}$ CONUS area percentiles.
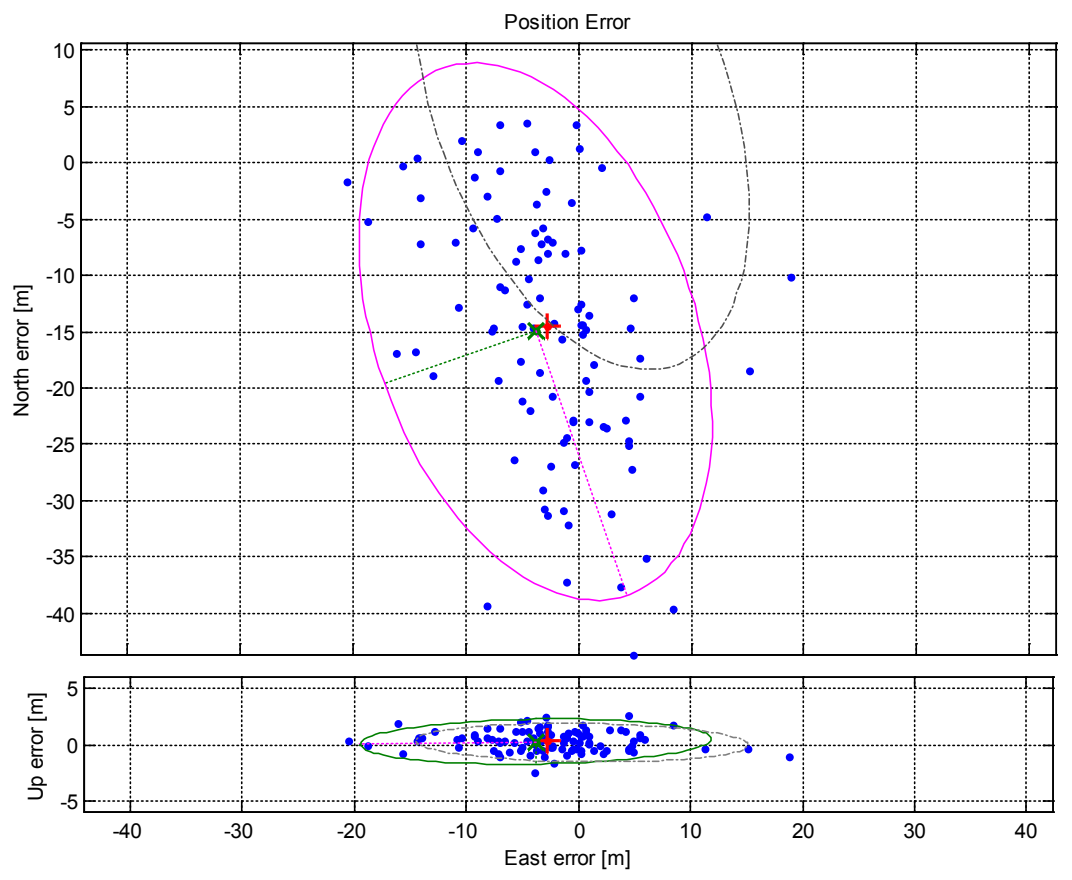

Figure 50: Position error pattern for Test Case E2; Monte Carlo analysis scatter plot (blue); Horizontal 90\% errors ellipse (top plot, magenta); Vertical 90\% errors ellipse (bottom plot, green); The dashed gray ellipses are the $90 \%$ error ellipses for base Test Case E0 that are shown here for comparison purposes.

In an attempt to assess performance sensitivity to ground station placement, Test Case E3 has a setup similar to that of Test Case B3 (Fig. 36), i.e., with an array of ground stations that are located along the US coastal and land borders. All other parameters are identical to those of Test Case E0 except for minor adjustments in signal frequencies that are necessary in order to maintain physical feasibility. The position error plot of Fig. 51 demonstrates the impact of using the alternative transmitters' setup on the position error distribution. Comparison with the Test Case E0 ellipses, which are shown in Fig. 51 as 
well, indicates that the position accuracy for Case E3 is significantly degraded. Errors for the a posteriori Chapman model's parameters are similarly larger for Test Case E3 for which the $h_{\text {sf }}$ scale height parameter estimation maps are presented in Fig. 52. The results are slightly inferior to those of Test Case E0 (Fig. 47). This assertion will be further validated in a later section via data in Table 6.
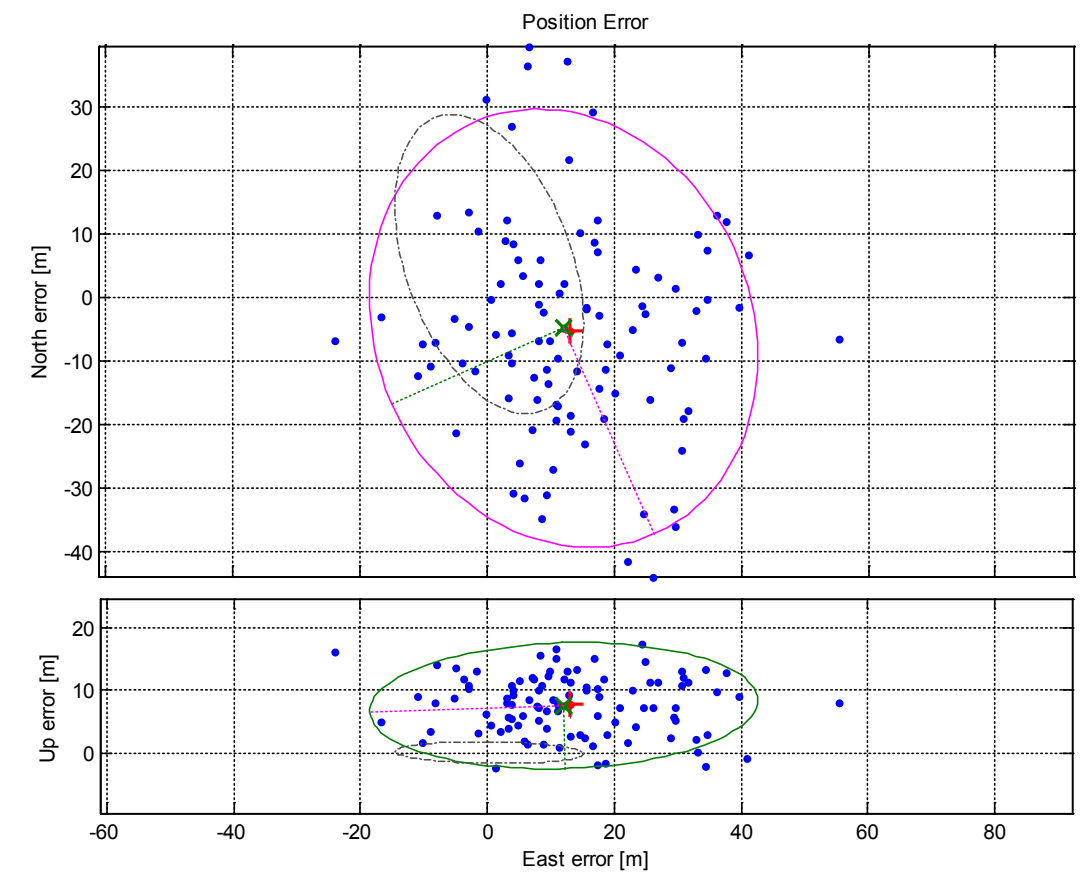

Figure 51: Position error pattern for Test Case E3; Monte Carlo analysis scatter plot (blue); Horizontal 90\% errors ellipse (top plot, magenta); Vertical 90\% errors ellipse (bottom plot, green); The dashed gray ellipses are the $90 \%$ error ellipses for base Test Case E0, shown here for comparison purposes.

The last test case studied in this group is Test Case E4, whose 1-sigma noise error is increased to 10 meters for the carrier phase measurements and kept unchanged at 1000 meters, for the range-equivalent group delay pseudoranges. A significant degradation in position accuracy in comparison to the base Test Case E0 is indicated by Fig. 53, which 

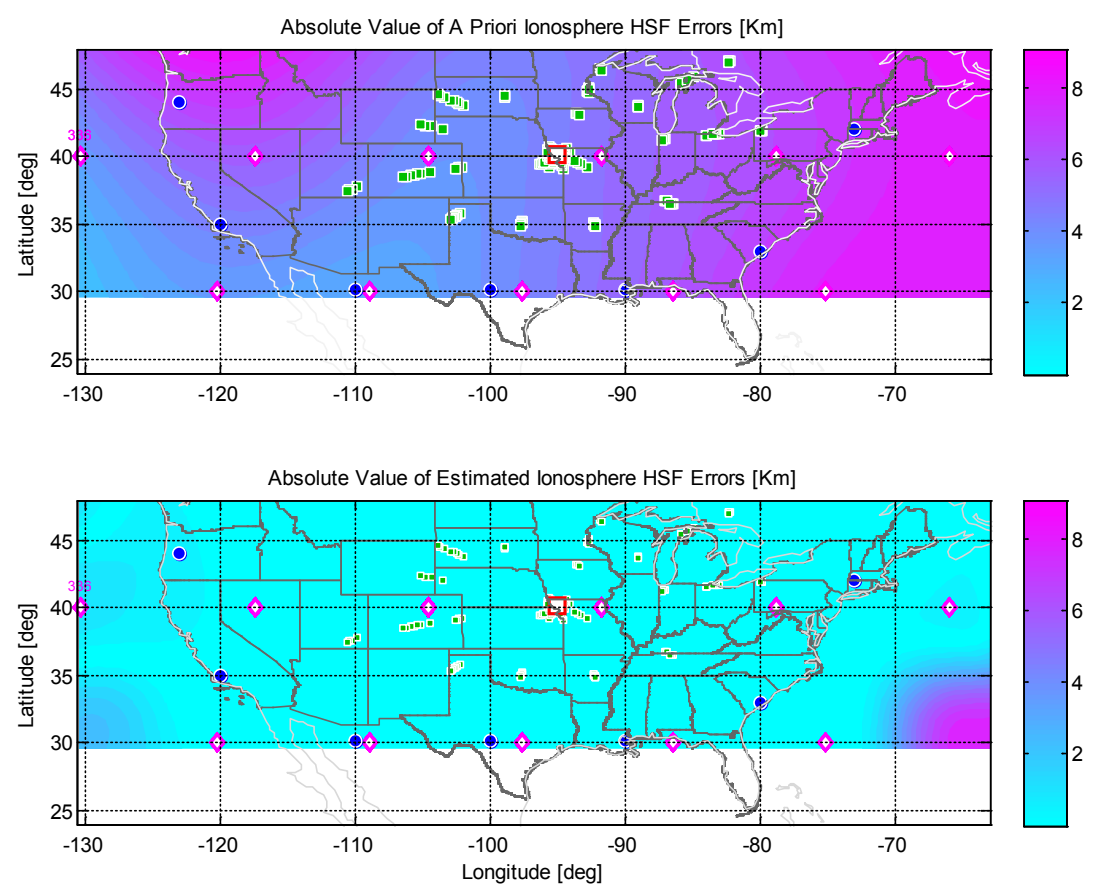

Figure 52: A priori (top) and a posteriori (bottom) errors for the ionospheric scale height $h_{\mathrm{sf}}$ parameter for a Nominal Scenario Mode run of Test Case E3.
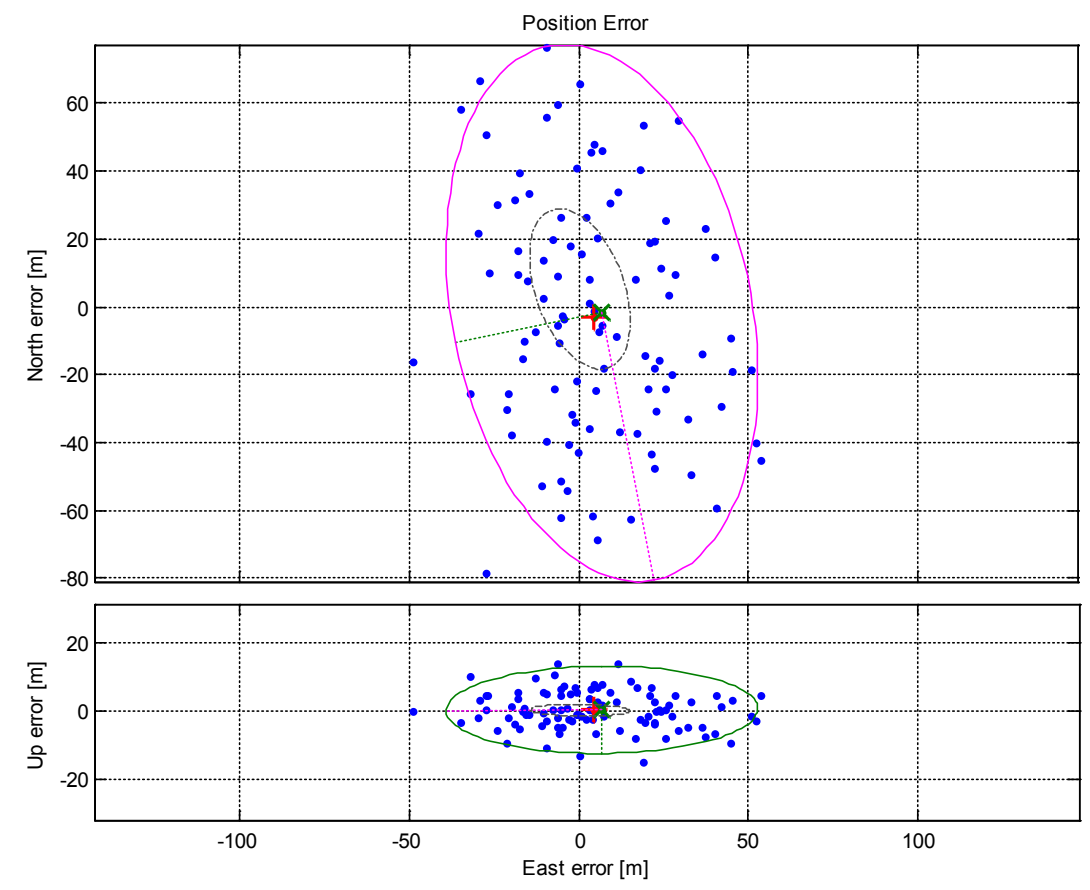

Figure 53: Position error pattern for Test Case E4; Monte Carlo analysis scatter plot (blue); Horizontal 90\% errors ellipse (top plot, magenta); Vertical 90\% errors ellipse (bottom plot, green); The dashed gray ellipses are the $90 \%$ error ellipses for base Test Case E0, shown here for comparison purposes. 
shows the horizontal $90 \%$ error ellipses for both cases. This trend is also evident in the $a$ posteriori errors for the ionospheric parameters.

\subsubsection{Test cases with a medium number of available ray-paths (Group D)}

This subsection presents the simulated performance for a scenario with a reduced number of available measurements. Test cases of Group D consider a set of ground stations that is a subset of the set of ground stations used with the high-availabilityscenario test cases of Group E. Each ground station is assumed to transmit between 1 and 3 signals with varying frequencies, so that the total number of ray-paths received at the receiver is 17 and the total number of measurements processed, including both group delay and beat carrier phase measurements, is 136 .

Test Case D0 is the base test case for the scenario with a medium number of available ray-paths. It uses the same truth and a priori ionosphere models as Test Case E0 and has the same Ionosphere Error Index $(\xi)$ of -0.2276 . The HF signals for this test case have frequencies in the range 3.0-6.0 MHz. The number of hops for each ray-path is 1-4, with a mixture of signals arriving from above and from below at the UE.

Figure 54 plots position errors obtained for a 100-run Monte Carlo analysis, with the usual notation and markings. The additional dashed gray ellipse in the top subplot marks the horizontal $90 \%$ error ellipse that has been obtained for Test Case E0. The impact of processing a smaller number of measurements is evident when the two ellipses are compared. The major and minor axes are about three times larger for the present scenario with a reduced number of available measurements. The mean horizontal position error for 
Test Case D0 is about 15 times larger than that of Test Case E0. Vertical accuracy is degraded as well, yet errors in the vertical direction are still sufficiently small for many navigation applications.

A comparison of Fig. 55, which shows the performance for the a posteriori estimates of the ionospheric parameters, with the equivalent Fig. 46 for Test Case E0 shows that residual VTEC estimation errors for the present case are about twice as large as for the high-number-of-available-signals test case. Even so, the a posteriori errors for all three sets of Chapman parameters have been reduced dramatically in comparison to their $a$ priori values for this case.

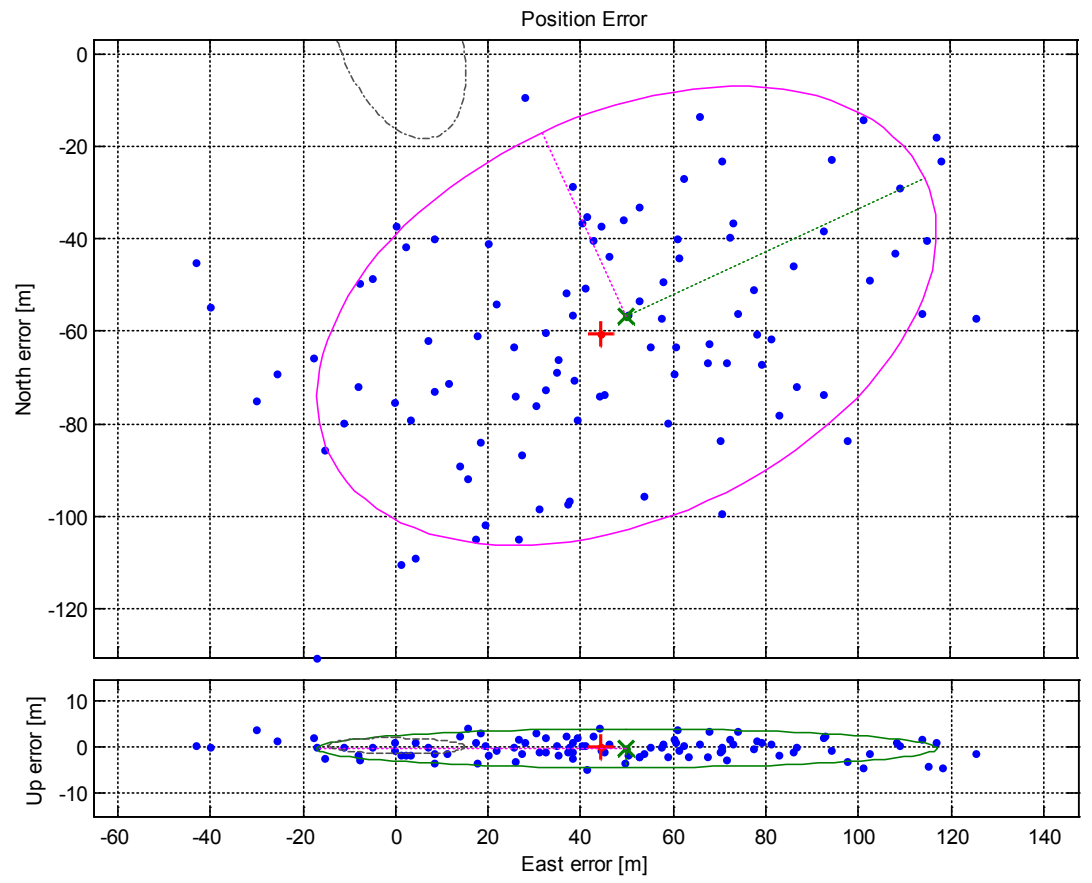

Figure 54: Position error pattern for Test Case D0; Monte Carlo analysis scatter plot (blue); Horizontal 90\% errors ellipse (top plot, magenta); Vertical 90\% errors ellipse (bottom plot, green); The dashed gray ellipses are the $90 \%$ error ellipse for the base test case of Group E (Test Case E0), shown here for comparison purposes. 

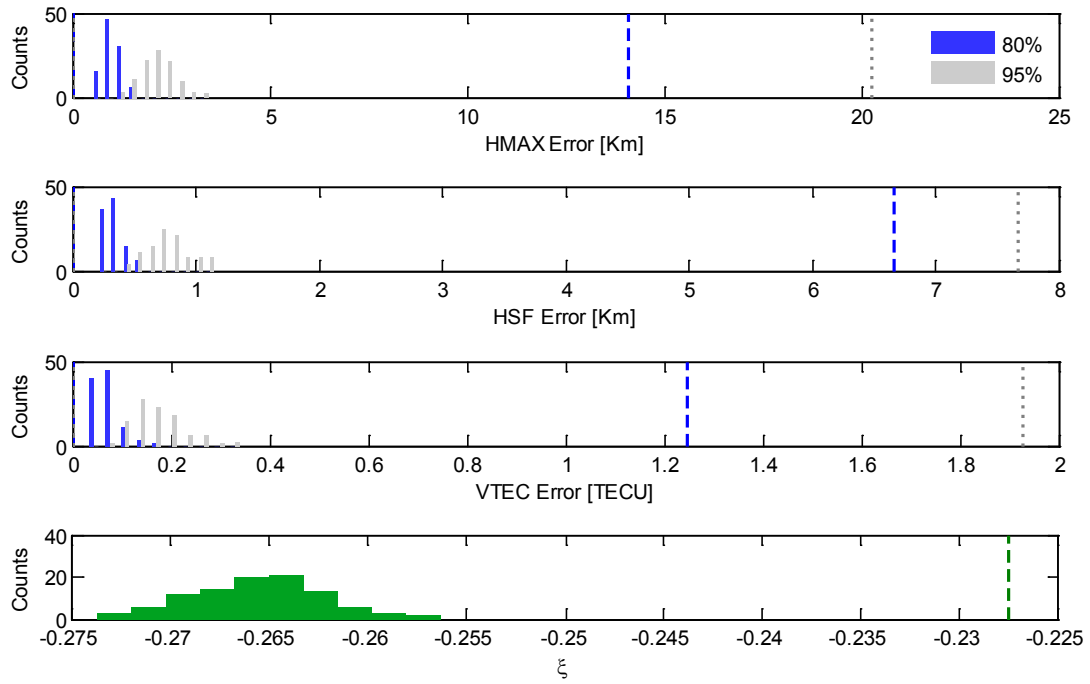

Figure 55: The statistical characteristics of the a posteriori ionosphere model errors for Test Case D0, including histograms of the $80^{\text {th }}$ and $95^{\text {th }}$ CONUS area percentile error values for the three Chapman parameters. The bottom panel is a histogram of $\xi$ values. Dashed lines mark the $80^{\text {th }}$ and $95^{\text {th }}$ percentile values of these quantities for the a priori ionosphere parameter error vector, except in the bottom panel the dashed line marks the a priori error's mean.

As with other test cases of significant IEI, the large-IEI variant, Test Case D2, is characterized by a mean horizontal position error that is significantly larger than that of the group's base test case. Note how the center of the $90 \%$ position error ellipse (Fig. 56) is located much further from zero compared to Test Case D0's that is also shown in this figure in the form of a (cropped) gray dashed ellipse. The centers of the two vertical $90 \%$ error ellipses that are shown in the bottom panel, however, are comparably close to zero in the local vertical direction. 


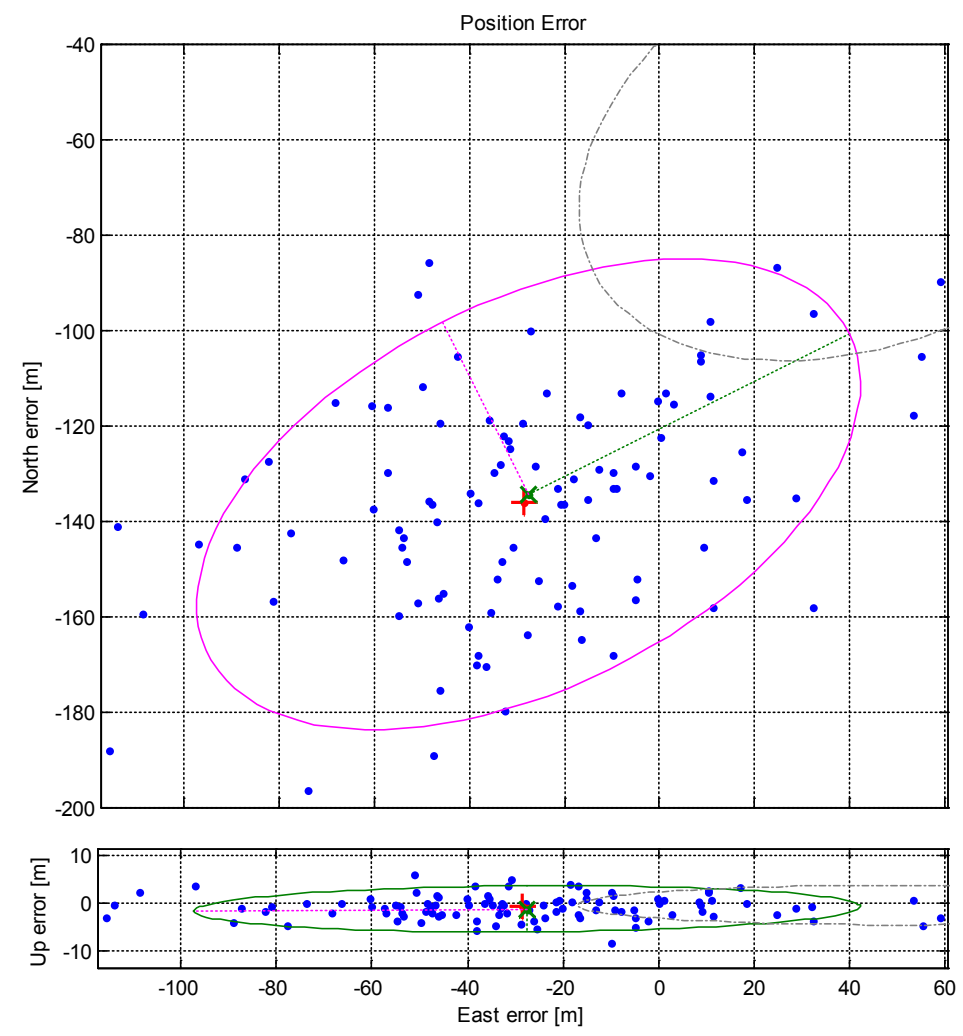

Figure 56: Position error pattern for Test Case D2; Monte Carlo analysis scatter plot (blue); Horizontal 90\% errors ellipse (top plot, magenta); Vertical 90\% errors ellipse (bottom plot, green); The dashed gray ellipses are the $90 \%$ error ellipses for base Test Case D0 and are included here for comparison purposes. 


\subsubsection{Summary of test cases using combined code and carrier ranging}

Table 5 summarizes the numerical results for the seven test cases presented in this section in terms of positioning accuracy. As before, location of the computed mean error is given in a local level north-east-vertical coordinate system whose origin is at the true location of the receiver. Horizontal position errors are expressed in term of a horizontal errors ellipse that bounds $90 \%$ of the computed east-north coordinates. The vertical error is characterized by its standard deviation.

\begin{tabular}{|c|c|c|c|c|c|c|}
\hline & & $\begin{array}{l}\text { base } \\
\text { case }\end{array}$ & $\begin{array}{l}\text { ray-paths } \\
\text { from } \\
\text { above only }\end{array}$ & $\begin{array}{c}\text { large } \\
\text { initial } \xi\end{array}$ & $\begin{array}{c}\text { alternative } \\
\text { stations } \\
\text { placement }\end{array}$ & $\begin{array}{c}\text { increased } \\
\text { meas. } \\
\text { noise }\end{array}$ \\
\hline \multirow{5}{*}{$\begin{array}{c}\text { Medium } \\
\text { number of } \\
\text { available } \\
\text { signals } \\
\text { Scenario } \\
\text { (Group D) }\end{array}$} & test case \# & DO & & D2 & & \\
\hline & $\begin{array}{l}90 \% \text { errors ellipse } \\
\text { semi-major axis }[\mathrm{m}]\end{array}$ & 71 & & 75 & & \\
\hline & $\begin{array}{l}90 \% \text { errors ellipse } \\
\text { semi-minor axis }[\mathrm{m}]\end{array}$ & 44 & & 40 & & \\
\hline & $\begin{array}{l}\text { vertical error STD } \\
{[\mathrm{m}]}\end{array}$ & 2 & & 2 & & \\
\hline & $\begin{array}{l}\text { mean error NEV } \\
{[\mathrm{m}, \mathrm{m}, \mathrm{m}]}\end{array}$ & $-57,50,0$ & & $-134,-28,-1$ & & \\
\hline \multirow{5}{*}{$\begin{array}{c}\text { High } \\
\text { number of } \\
\text { available } \\
\text { signals } \\
\text { Scenario } \\
\text { (Group E) }\end{array}$} & test case \# & EO & E1 & $E 2$ & E3 & E4 \\
\hline & $\begin{array}{l}90 \% \text { errors ellipse } \\
\text { semi-major axis }[\mathrm{m}]\end{array}$ & 25 & 18 & 24 & 35 & 80 \\
\hline & $\begin{array}{l}90 \% \text { errors ellipse } \\
\text { semi-minor axis }[\mathrm{m}]\end{array}$ & 13 & 9 & 14 & 30 & 44 \\
\hline & $\begin{array}{l}\text { vertical error STD } \\
{[\mathrm{m}]}\end{array}$ & 1 & 5 & 1 & 5 & 6 \\
\hline & $\begin{array}{l}\text { mean error NEV } \\
{[\mathrm{m}, \mathrm{m}, \mathrm{m}]}\end{array}$ & $5,0,0$ & $-2,2,-1$ & $-15,-4,0$ & $-5,12,8$ & $-2,7,0$ \\
\hline
\end{tabular}

Table 5: Class 2 test cases' primary position error characteristics.

A summary of performance for ionosphere model correction is presented in Table 6 . The first three rows for each category consist of the a priori (upper the pair in a given cell) and the mean of the a posteriori (lower of the pair in a given cell) estimation errors of the three Chapman parameters. For each pair of values on a line of a cell, the left refers 
to the $80^{\text {th }}$ CONUS area percentile value, and the right to the $95^{\text {th }}$ percentile value. The fourth row shows the a priori and the mean a posteriori IEI values that were computed for each test case.

\begin{tabular}{|c|c|c|c|c|c|c|c|}
\hline & & & $\begin{array}{l}\text { base } \\
\text { case }\end{array}$ & $\begin{array}{l}\text { ray-paths } \\
\text { from }\end{array}$ & $\begin{array}{c}\text { large } \\
\text { initial } \xi\end{array}$ & $\begin{array}{l}\text { alternative } \\
\text { stations }\end{array}$ & $\begin{array}{c}\text { increased } \\
\text { meas. }\end{array}$ \\
\hline & test case \# & & Do & & D2 & & \\
\hline Medium & $\begin{array}{l}h_{\max } 80^{\text {th }} / 95^{\text {th }} \\
\text { percentile }[\mathrm{km}]\end{array}$ & $\begin{array}{c}\text { a priori } \\
\text { mean } \text { a post. }\end{array}$ & $\begin{array}{l}14.1 / 20.2 \\
0.84 / 1.93\end{array}$ & & $\begin{array}{l}20.4 / 34.9 \\
1.53 / 3.62\end{array}$ & & \\
\hline number of & $h_{\text {sf }} 80^{\text {th }} / 95^{\text {th }}$ & a priori & $6.7 / 7.7$ & & $16.5 / 18.3$ & & \\
\hline $\begin{array}{c}\text { available } \\
\text { signals }\end{array}$ & percentile $[\mathrm{km}]$ & mean a post. & $0.29 / 0.62$ & & $0.38 / 0.67$ & & \\
\hline Scenario & $V T E C 80^{\text {th }} / 95^{\text {th }}$ & a priori & $1.25 / 1.93$ & & $1.75 / 3.02$ & & \\
\hline (Group D) & percentile [TECU] & mean a post. & $0.05 / 0.13$ & & $0.10 / 0.31$ & & \\
\hline & $\xi$ & a priori & -0.2276 & & 0.0848 & & \\
\hline & test case \# & & EO & E1 & $E 2$ & E3 & E4 \\
\hline & $\begin{array}{l}h_{\max } 80^{\text {th }} / 95^{\text {th }} \\
\text { percentile }[\mathrm{km}]\end{array}$ & $\begin{array}{c}\text { a priori } \\
\text { mean } \text { a post. }\end{array}$ & $\begin{array}{l}14.1 / 20.2 \\
0.42 / 0.88\end{array}$ & $\begin{array}{l}14.1 / 20.2 \\
0.28 / 0.63\end{array}$ & $\begin{array}{l}20.4 / 35.0 \\
0.88 / 1.72\end{array}$ & $\begin{array}{l}13.1 / 19.5 \\
0.33 / 1.96\end{array}$ & $\begin{array}{l}14.1 / 20.2 \\
0.85 / 1.53\end{array}$ \\
\hline number of & & & $67 / 7$ - & $67 / 77$ & $165 / 183$ & $76 / 8 ?$ & \\
\hline available & percentile $[\mathrm{km}]$ & mean a post. & $0.30 / 0.77$ & $0.17 / 0.68$ & $0.43 / 1.39$ & $0.35 / 1.78$ & $0.39 / 0.85$ \\
\hline Scenario & VTEC $80^{\text {th }} / 95^{\text {th }}$ & a priori & $1.25 / 1.93$ & $1.25 / 1.93$ & $1.7 / 3.0$ & $1.56 / 2.22$ & $1.25 / 1.93$ \\
\hline (Group E) & percentile [TECU] & mean a post. & $0.04 / 0.09$ & $0.02 / 0.09$ & $0.11 / 0.33$ & $0.07 / 0.36$ & $0.06 / 0.13$ \\
\hline & $\xi$ & a priori & -0.2276 & -0.2276 & 0.0848 & -0.2165 & -0.2276 \\
\hline
\end{tabular}

Table 6: Class 2 test cases' primary ionosphere model errors characteristics. 


\subsection{Test Cases Using Group Delay Only, Random Ionosphere Model (Class 3)}

The various test cases that have been presented in the previous sections provide data for well-defined scenarios which are characterized by constant true and a priori ionosphere models that have a constant difference between them. The analysis is expanded here and in the next section. The test cases used here are very similar to the test cases that have been presented so far, except they use a statistical approach to analyze the expected effects of random errors between the a priori and true ionosphere models.

\subsubsection{Test cases with a high number of available ray-paths (Group F)}

Test Case C0 which was presented in Section 7.3.3 exhibited the highest level of positioning accuracy of all test cases for which only group delay measurements are used and measurement noise of $1 \mathrm{~km}$ is assumed (See Table 2). With an initial IEI parameter of -0.2276 that is close to the IEI's distribution mean $(-0.25)$, its a priori ionosphere model errors can be regarded as 'average' in the sense that the likelihood of encountering errors of such magnitude is high. A broader perspective on performance is obtained by expending the scope of this test case such that it's a priori ionospheric model errors are taken as a random vector rather than a constant one. This setup, that constitutes Test Case F0, is characterized with the same combination of parameters as that of Test Case C0 except for its ionospheric model. The considered a priori error model utilizes a covariance matrix of the form $\gamma M_{0}$. The scaling factor $\gamma$ takes five different values: $1,0.5$, $0.1,0.001$ and 1e-9. The first case for which $\gamma=1$ is a worst case scenario where the uncertainty of the ionosphere model is assumed as big as the ionospheric diversity matrix 
$M_{0}$. The last case can be regarded as the case of extremely good knowledge of the ionosphere.

Figure 57 plots the horizontal and vertical 90\% error ellipses for Test Case F0. These have been generated with the procedure described in Subsection 7.2.2. The two sets of five ellipses correspond with the different values of $\gamma$. For the case of $\gamma=10^{-9}$, the resulting position errors are solely due to ranging errors. It is evident that vertical accuracy is only somewhat sensitive to $\gamma$.

Position error results can now be compared to the results that have been obtained for the fixed-ionosphere-model base Test Case $\mathrm{C} 0$. The position error pattern in that case is characterized by a fixed-ionosphere-induced mean error that is relatively small compared to the effect of measurement-noise-induced errors. The resulting position error pattern shown in Fig. 43 is similar to that of Test Case F0, and in particular, to the case of $\gamma=0.1$ in Fig. 57.

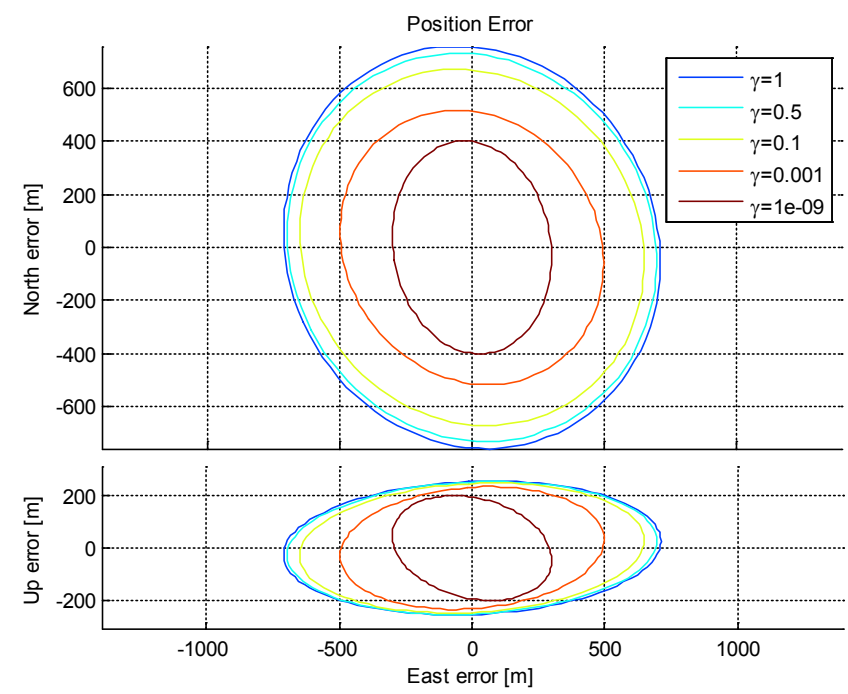

Figure 57: Horizontal and vertical 90\% error ellipses for Test Case F0 with different values of $\gamma$. 
Figure 58 presents the ten $80^{\text {th }}$ percentile value maps above CONUS for the ionospheric peak electron density height parameter $h_{\max }$ for Test Case F0. Note that the information which is presented in these plots (and in those to follow) is very different from the information which has been presented in figures such as Fig. 25. The left column shows $80^{\text {th }}$ percentile maps for the a priori estimates of this parameter while the right column consists of plot for its a posteriori estimates. Each row is associated with a different value of $\gamma$, so that the top row is associated with $\gamma=1$ (i.e., high uncertainty for the ionosphere model) and the bottom row is associated with $\gamma=10^{-9}$ which is the case of a very accurate a priori ionosphere model. Note the different color-code scales for the ten maps of Fig. 58. This approach has been favored over using the same color-code scales for neighboring panels in an effort to keep the map plots as informative as possible. The reader should notice that in all cases, for a given row, the right-hand plot of the $a$ posteriori difference map is characterized by values that are significantly smaller than those of the left-hand plot that presents the a priori difference map.

As expected, smaller $80^{\text {th }}$ percentile values for the $h_{\text {max }}$ parameter have been computed for smaller values of $\gamma$, as shown in Fig. 58 that presents all ten $80^{\text {th }}$ percentile error maps for that parameter. For the first four cases of $\gamma$, a significant reduction in the $80^{\text {th }}$ percentile values for the a posteriori estimates is evident. For $\gamma=0.5$, values in the range $30-35 \mathrm{~km}$ have been reduced to about 2-4 km. For the case of $\gamma=10^{-9}$, however, the initial $80^{\text {th }}$ percentile value are very small and so is the difference between a priori and $a$ posteriori values. 

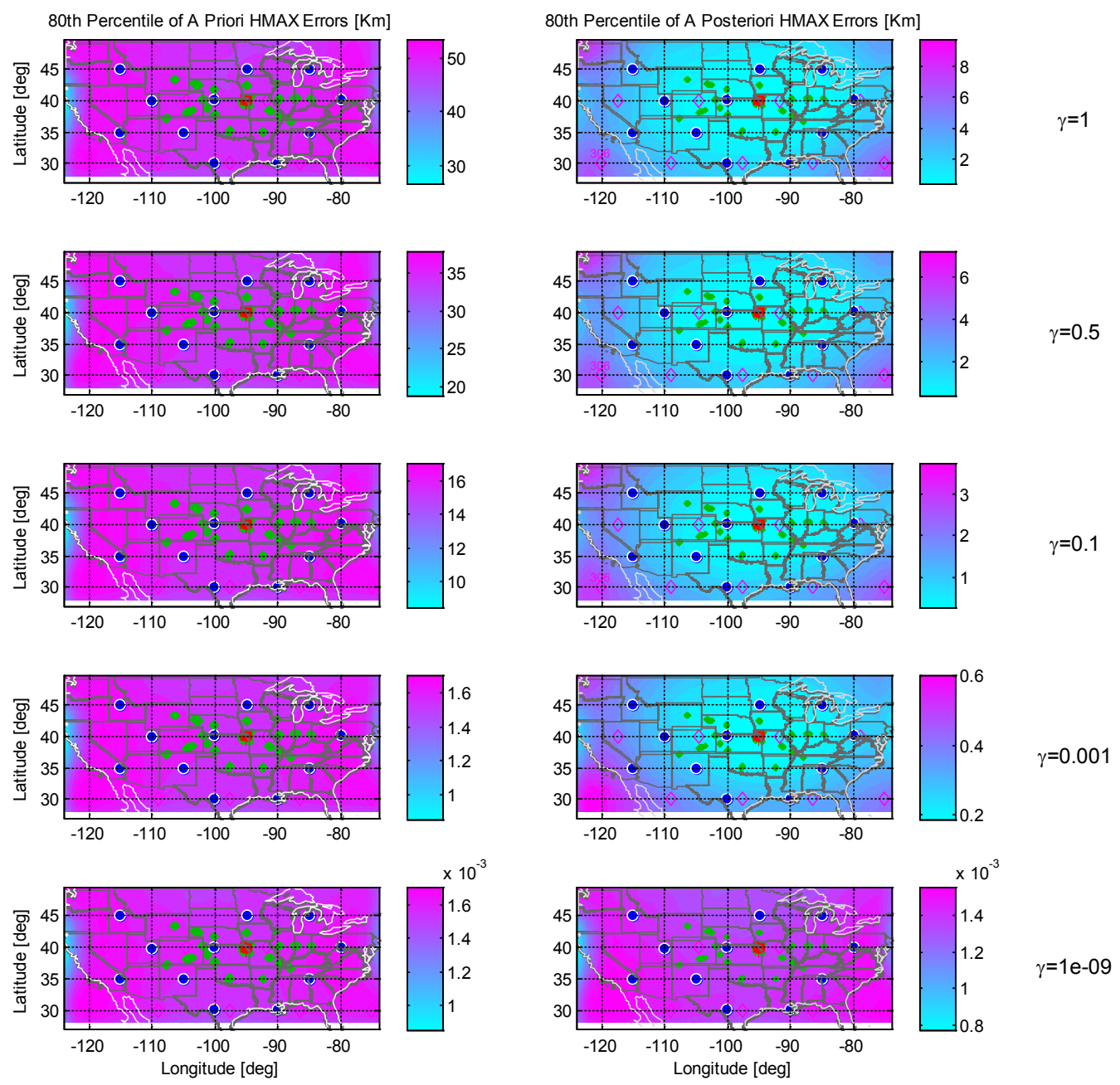

Figure 58: Eightieth percentile value maps for the a priori (left column) and a posteriori (right column) estimates' errors for the ionospheric peak electron density height parameter $h_{\max }$ for Test Case F0. Each row corresponds to a different value of $\gamma$.

Similar trends have been observed for the ionospheric scale height parameter $h_{\mathrm{sf}}$, shown in Fig. 59. It is also notable that the smallest a posteriori $80^{\text {th }}$ percentile values are obtained in the region that is close to the location of the receiver, especially in the cases of large $\gamma$ 's. 

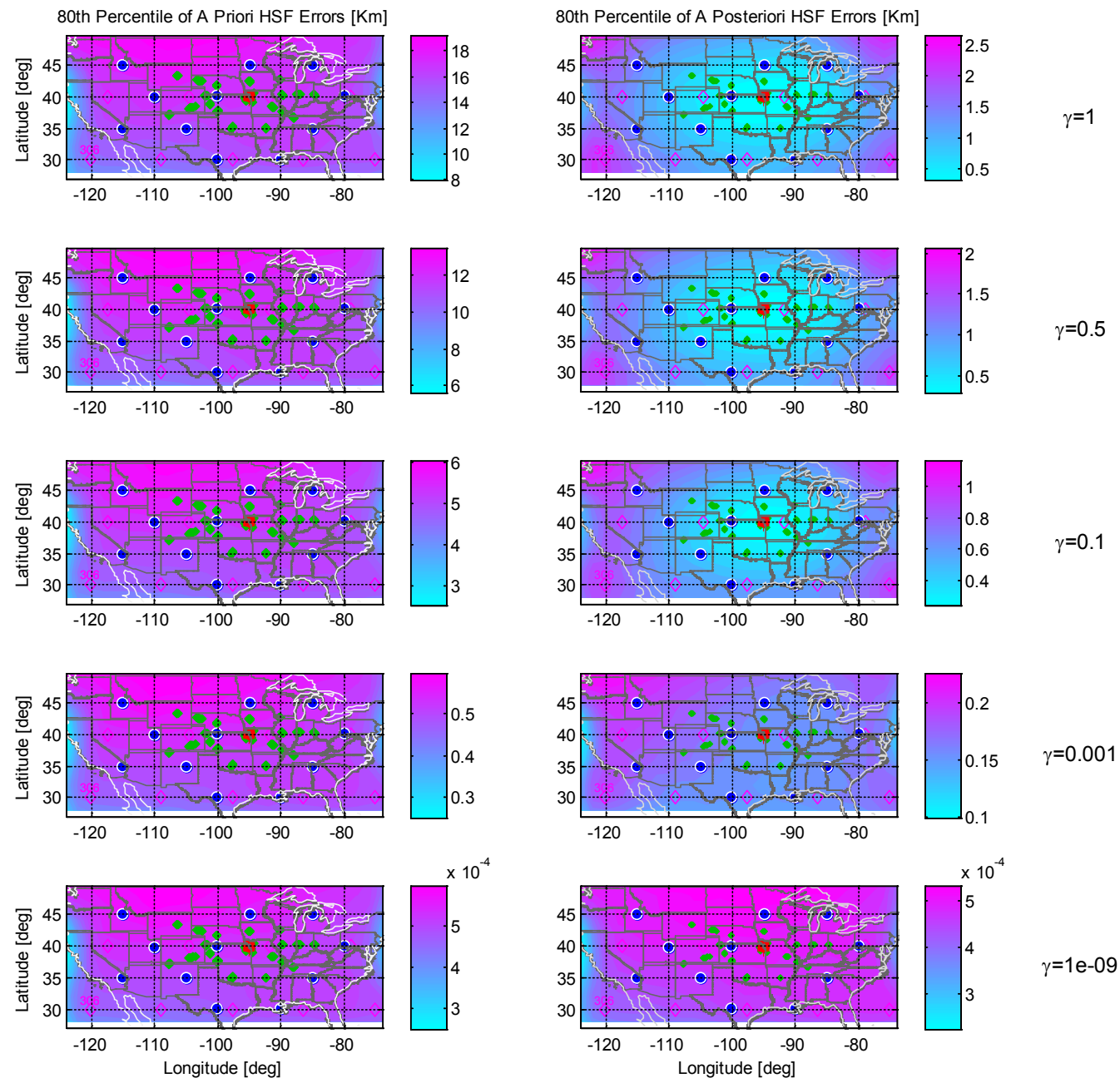

Figure 59: Eightieth percentile value maps for the a priori (left column) and a posteriori (right column) estimates' errors for the ionospheric scale height parameter $h_{\mathrm{sf}}$ for Test Case F0. Each row corresponds to a different value of $\gamma$.

However, the trend to have the lowest error nearest to the receiver is not as prominent with the results for the VTEC parameter, as it can be inferred from the maps shown in Fig. 60. Regardless, a significant reduction in $80^{\text {th }}$ percentile values is evident for all cases of $\gamma$ for this parameter as well. 


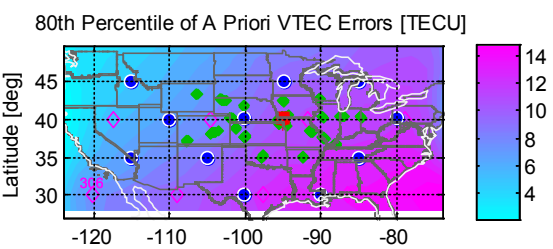

80th Percentile of A Posteriori VTEC Errors [TECU]

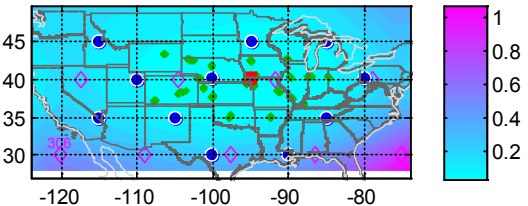

$\gamma=1$
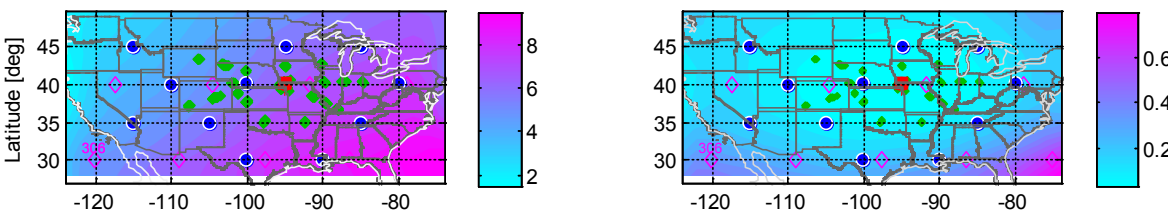

$\gamma=0.5$
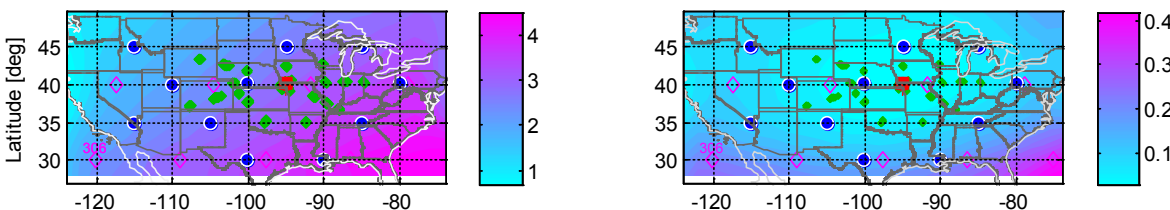

$\gamma=0.1$
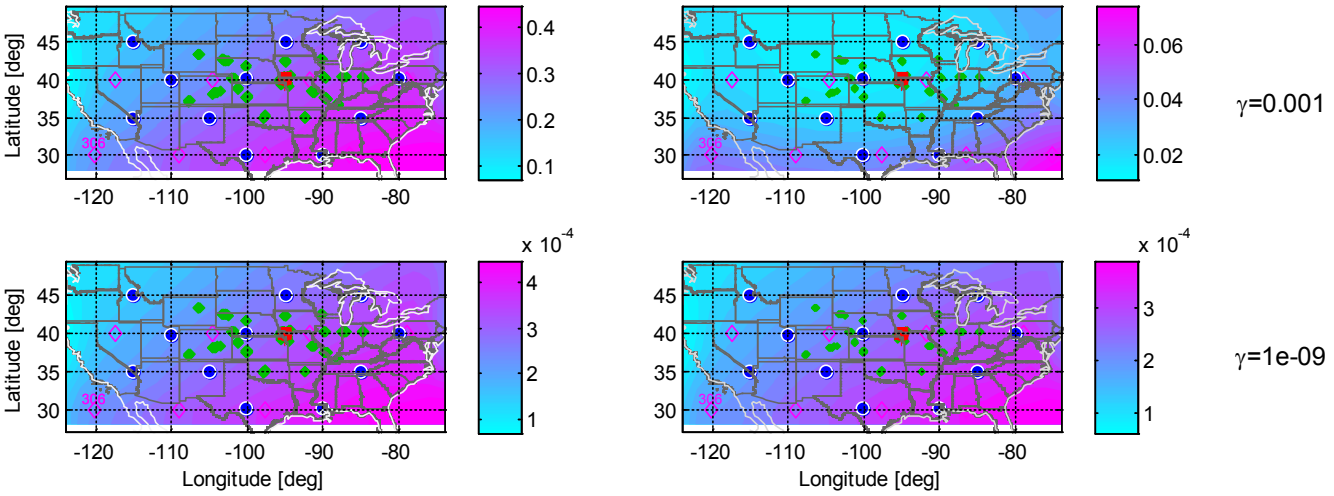

Figure 60: Eightieth percentile value maps for the a priori (left column) and a posteriori (right column) estimates' errors for the ionospheric vertical total electron content parameter $V T E C$ for Test Case F0. Each row corresponds to a different value of $\gamma$. 


\subsection{Test Cases Using Combined Code and Carrier Phase Ranging, Random Ionosphere Model (Class 4)}

This section presents the performance for the batch-filtering algorithm that utilizes a combined group-delay/beat-carrier-phase configuration, in the presence of random ionosphere model errors. The studied test cases in this group are in fact the statisticalanalysis variants of Class 2's test cases that have been presented in Section 7.4.

Table 7 summarizes the primary characteristics for all five Class 4 test cases analyzed in this section: their standard deviation for both group delay and beat carrier phase measurement noise, number of ray-paths, direction of signals as they arrive at the receiver, and the placement pattern for the ground stations from which received signals were broadcast. As in the previous section, each test case is studied with five different values for the covariance matrix scaling factor $\gamma$.

\begin{tabular}{|c|c|c|c|c|c|}
\hline \multirow{6}{*}{$\begin{array}{c}\text { Medium } \\
\text { number of } \\
\text { available } \\
\text { signals } \\
\text { Scenario } \\
\text { (Group G) }\end{array}$} & & $\begin{array}{l}\text { base } \\
\text { case }\end{array}$ & $\begin{array}{c}\text { ray-paths } \\
\text { from } \\
\text { above only }\end{array}$ & $\begin{array}{c}\text { alternative } \\
\text { stations } \\
\text { placement }\end{array}$ & $\begin{array}{l}\text { increased } \\
\text { meas. noise }\end{array}$ \\
\hline & test case \# & G0 & & & \\
\hline & meas. noise $\sigma[\mathrm{m}]$ & $1000 / 1$ & & & \\
\hline & ray-paths & $17 \times 4$ & & & \\
\hline & $\begin{array}{l}\text { wave arrival } \\
\text { direction }\end{array}$ & both & & & \\
\hline & stations placement & grid & & & \\
\hline \multirow{5}{*}{$\begin{array}{c}\text { High } \\
\text { number of } \\
\text { available } \\
\text { signals } \\
\text { Scenario } \\
\text { (Group H) }\end{array}$} & test case \# & $\mathrm{HO}$ & $H 1$ & $H 3$ & $H 4$ \\
\hline & meas. noise $\sigma[\mathrm{m}]$ & $1000 / 1$ & $1000 / 1$ & $1000 / 1$ & $1000 / 10$ \\
\hline & ray-paths & $32 \times 4$ & $32 \times 4$ & $32 \times 4$ & $32 \times 4$ \\
\hline & $\begin{array}{l}\text { wave arrival } \\
\text { direction }\end{array}$ & both & above only & both & both \\
\hline & stations placement & grid & grid & perimeter & grid \\
\hline
\end{tabular}

Table 7: Setup configuration for two groups and five test cases of Class 4. 


\subsubsection{Test cases with a high number of available ray-paths (Group H)}

The base Test Case $\mathrm{H} 0$ is the random-ionosphere equivalent of Test Case E0. It considerers the same array of ground stations, ray-paths and receiver location. Group delay and beat carrier phase measurement noise are similarly identical to those of Test Case E0.

Figure 61 plots the horizontal and vertical $90 \%$ error ellipses for this test case. As before, the two sets of five ellipses correspond with the different values of $\gamma$, where for the case of $\gamma=10^{-9}$, the resulting position errors are solely due to ranging errors. Vertical accuracy appears almost indifferent to $\gamma$. The horizontal $90 \%$ error ellipses are significantly smaller than their Test Case F0 equivalents. In particular, for $\gamma=0.001$ and smaller, the remarkably small errors indicate that if there were some sort of ionosphere monitoring network, then this system might be very accurate.

Position error results can be compared to the results that have been obtained for the fixed-ionosphere-model base Test Case E0. The position error pattern in that case is characterized by a fixed-ionosphere-induced mean error that is relatively small compared to measurement-noise-induced errors. The resulting pattern is very similar to that of the present Test Case $\mathrm{H} 0$ for the case of $\gamma=0.1$ to $\gamma=0.5$ - see Fig. 45. 


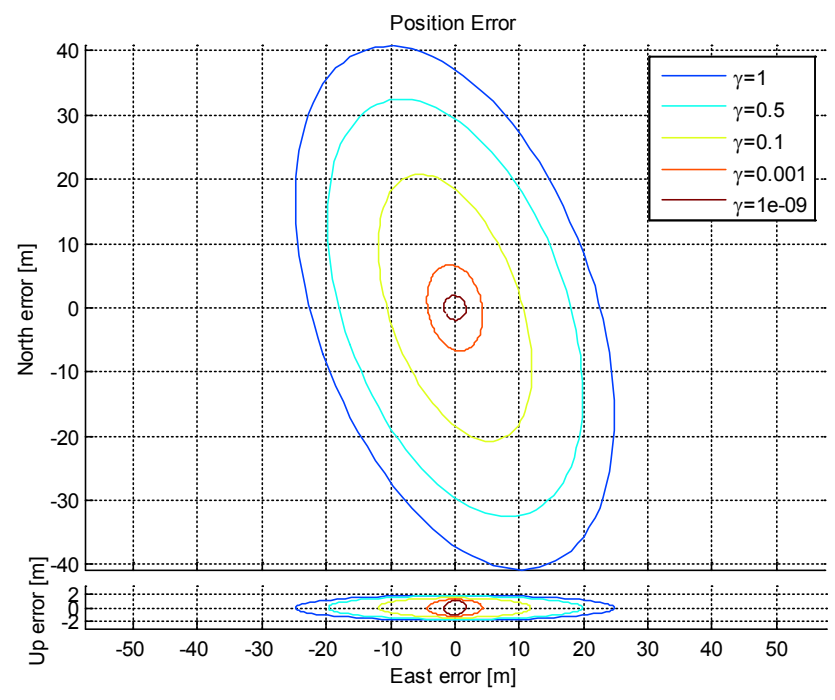

Figure 61: Horizontal and vertical 90\% error ellipses for Test Case H0 with different values of $\gamma$.

Eightieth percentile error maps for the three Chapman parameters are shown in the next three figures. For the peak electron density height $h_{\max }$ parameter map, shown in Fig. 62 , residual errors are about half of those computed for the group-delay-measurementsonly base test case, Test Case F0. Residual errors for the scale height parameter $h_{\mathrm{sf}}$ parameter (Fig. 63) exhibit similar characteristics in terms of the relative reduction in magnitude when compared to their a priori values and in comparison with Test Case F0. 


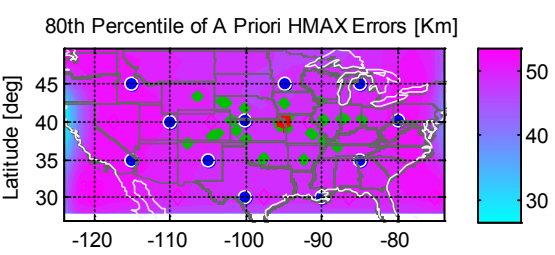

80th Percentile of A Posteriori HMAXErrors [Km]
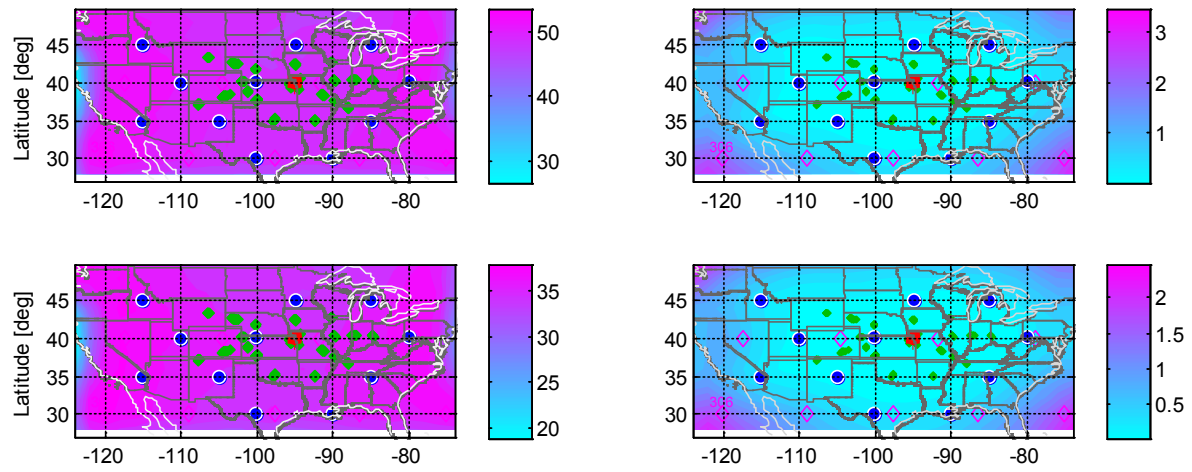

$\gamma=0.5$
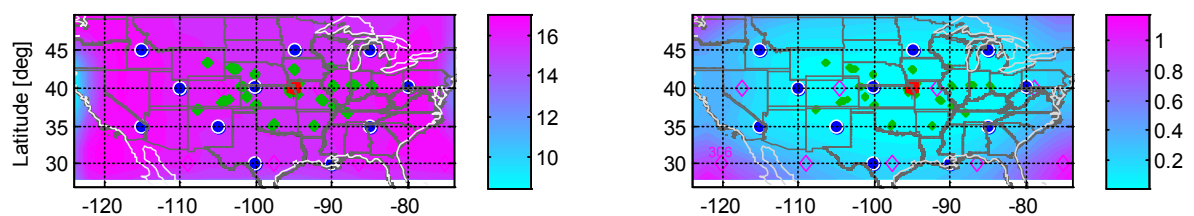

$\gamma=0.1$
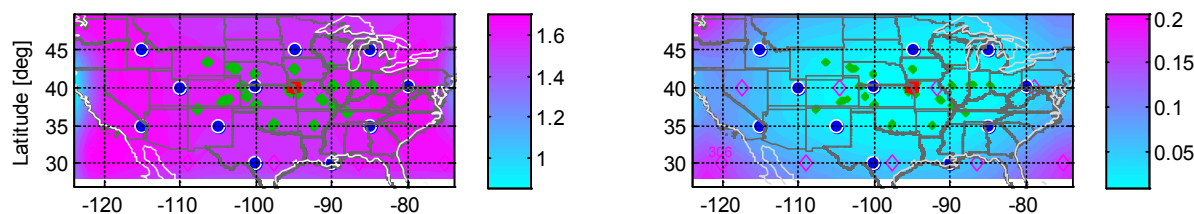

$\gamma=0.001$
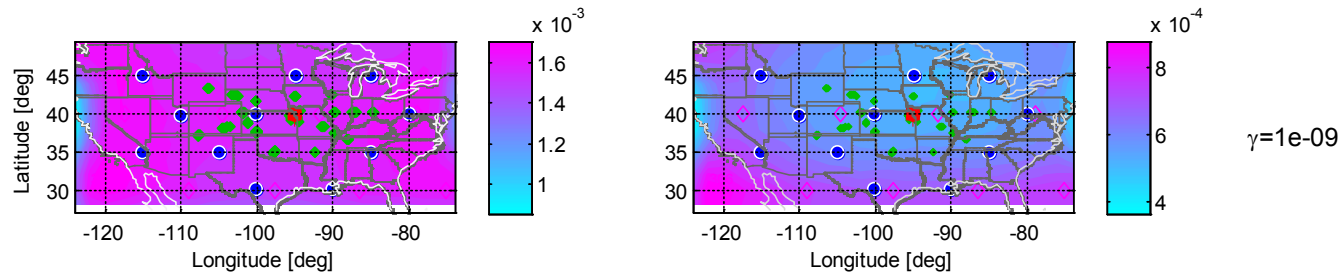

Figure 62: Eightieth percentile value maps for the a priori (left column) and a posteriori (right column) estimates' errors for the ionospheric peak electron density height parameter $h_{\max }$ for Test Case H0. Each row corresponds to a different value of $\gamma$. 

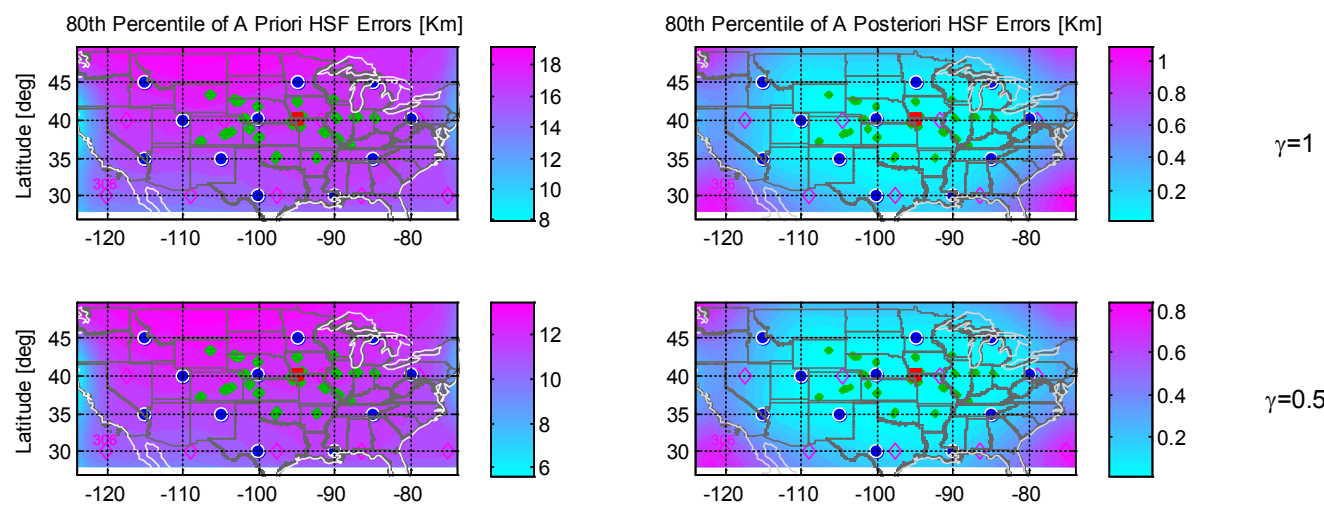

$\gamma=0.5$
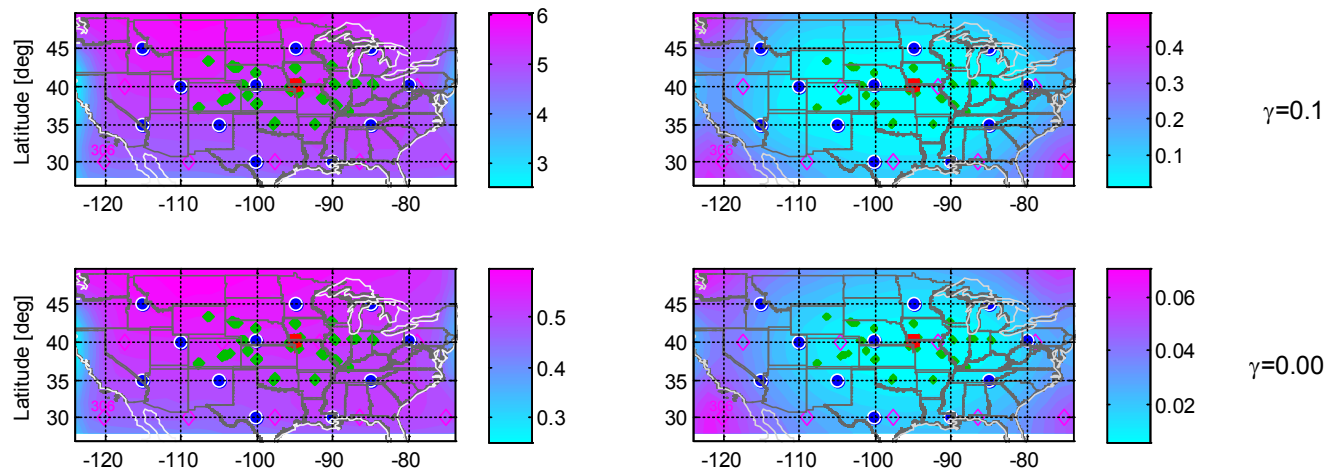

$\gamma=0.001$
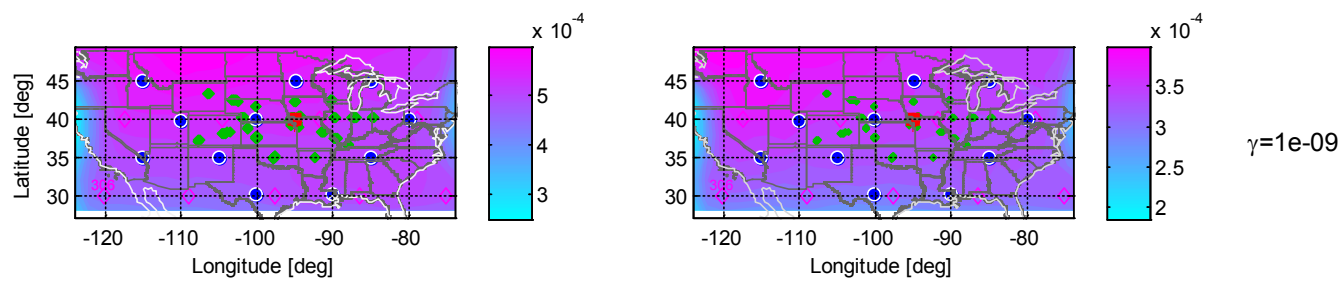

Figure 63: Eightieth percentile value maps for the a priori (left column) and a posteriori (right column) estimates' errors for the ionospheric scale height parameter $h_{\text {sf }}$ for Test Case H0. Each row corresponds to a different value of $\gamma$.

Consistent with these results, a significant reduction in the magnitude of the residual errors has been observed for the ionospheric vertical total electron content parameter VTEC (Fig. 64). In the worst case scenario of $\gamma=1$, residual errors, in terms of the $80^{\text {th }}$ percentile, are bounded by a value of 0.15 TECU. 

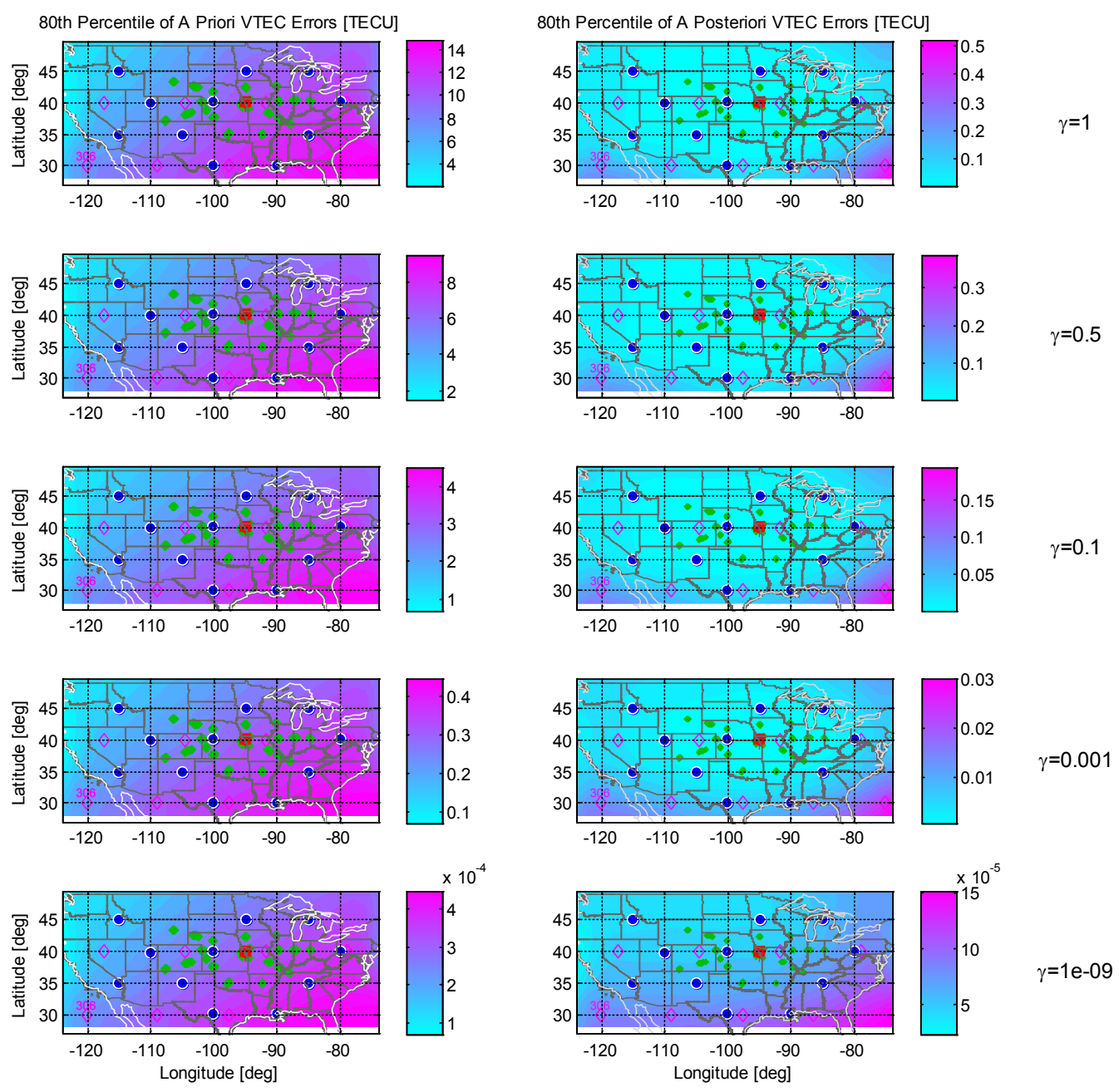

Figure 64: Eightieth percentile value maps for the a priori (left column) and a posteriori (right column) estimates' errors for the ionospheric vertical total electron content parameter $V T E C$ for Test Case H0. Each row corresponds to a different value of $\gamma$.

Test Case $\mathrm{H} 1$ is a variant of Test Case $\mathrm{H} 0$ that differs in its ray-path geometry. Here, ray-paths are configured such that all signals approach the receiver from above, consistent with similar test cases that have been discussed before. Minor modifications to the signals' frequencies were made in order to maintain the test case's physical feasibility. All other parameters were kept identical to those of the base configuration. 
Figure 65 plots the horizontal and vertical 90\% error ellipses for Test Case H1 using Eq. (100) for the five different $M$ values that result from the five different $\gamma$ values, as discussed in Section 7.2.2. The dashed gray ellipses are the $90 \%$ percentile ellipses for Test Case $\mathrm{H} 0$ in the case of $\gamma=1$, shown here for reference. As with the fixed-ionosphere test cases of Class 2, horizontal positioning accuracy has improved, while significant degradation in vertical accuracy is apparent. Comparing the results for Test Case H1 with the equivalent fixed-ionosphere-model test case, Test Case E1, it can be inferred that errors for the latter test case have a position error distribution similar to that of the former with a value of $\gamma$ between 0.1 and 0.5 .

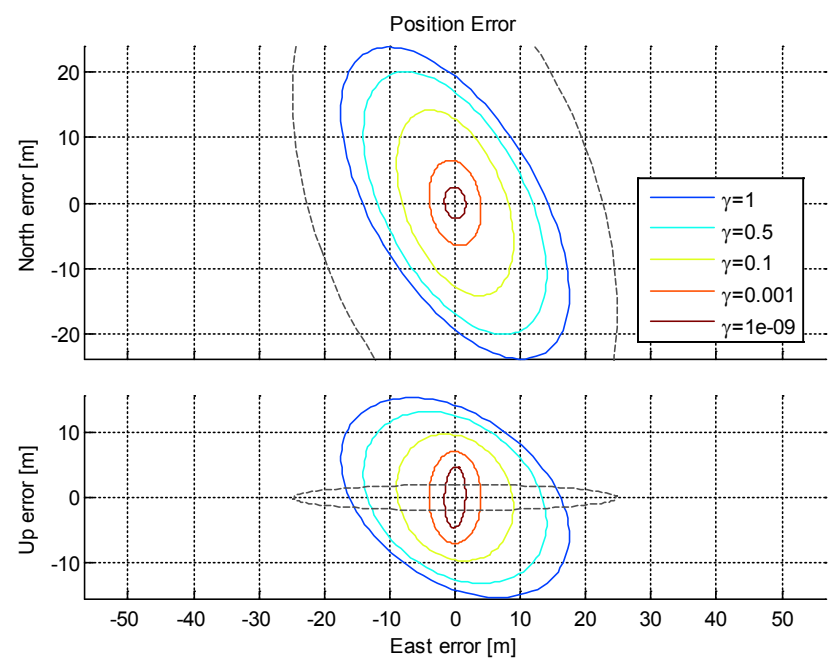

Figure 65: Horizontal and vertical 90\% error ellipses for Test Case H1 with different values of $\gamma$. The dashed ellipses in gray are the $90 \%$ error ellipses for Test Case H0 with $\gamma=1$.

Eightieth percentile error maps for the three Chapman parameters are shown in the next three figures. Consistent with the improved positioning accuracy, for the peak electron density height $h_{\max }$ parameter map, shown in Fig. 66, residual errors are between 


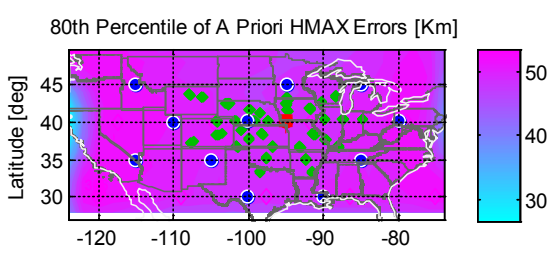

80th Percentile of A Posteriori HMAX Errors [Km]

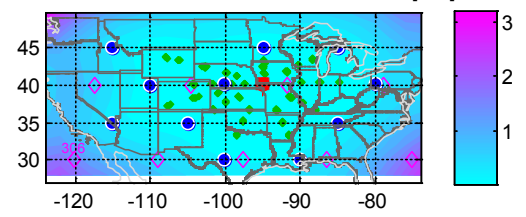

$\gamma=1$
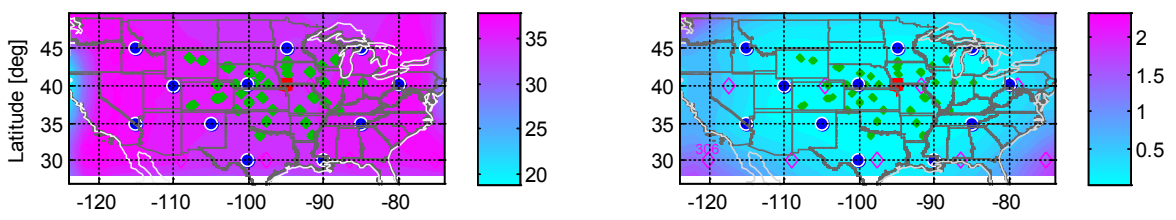

$\gamma=0.5$
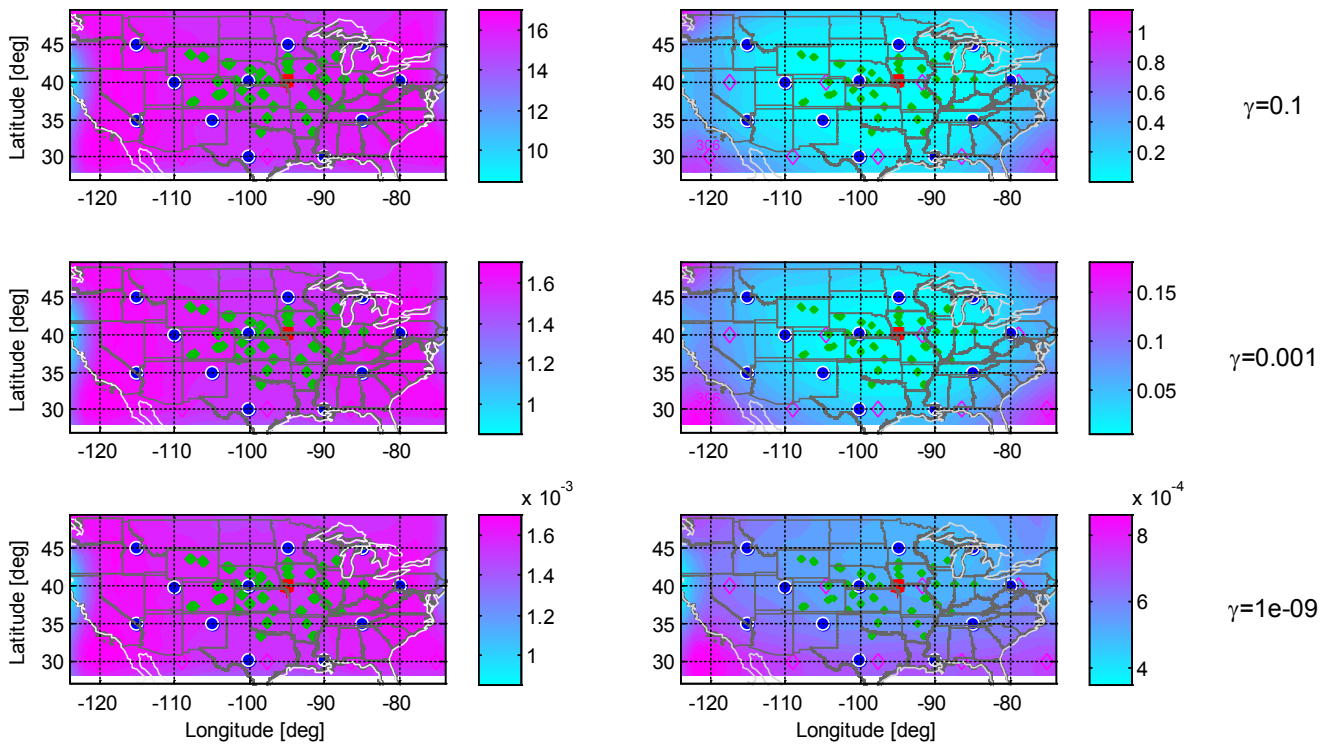

Figure 66: Eightieth percentile value maps for the a priori (left column) and a posteriori (right column) estimates' errors for the ionospheric peak electron density height parameter $h_{\max }$ for Test Case H1. Each row corresponds to a different value of $\gamma$.

$1 / 2$ and $3 / 4$ of those computed for the base Test Case H0. Residual errors for the scale height parameter $h_{\text {sf }}$ parameter (Fig. 67) exhibit similar characteristics in terms of the relative reduction in magnitude when compared to their a priori values and in comparison with the residual errors that have been recorded for Test Case H0. As shown in Fig. 68, residual errors for the ionospheric vertical total electron content parameter VTEC are similarly smaller than with Test Case H0, by roughly $30 \%$. In the worst case 
scenario of $\gamma=1$, residual errors above CONUS, in terms of the $80^{\text {th }}$ percentile, are bounded by a value of 0.1 TECU.
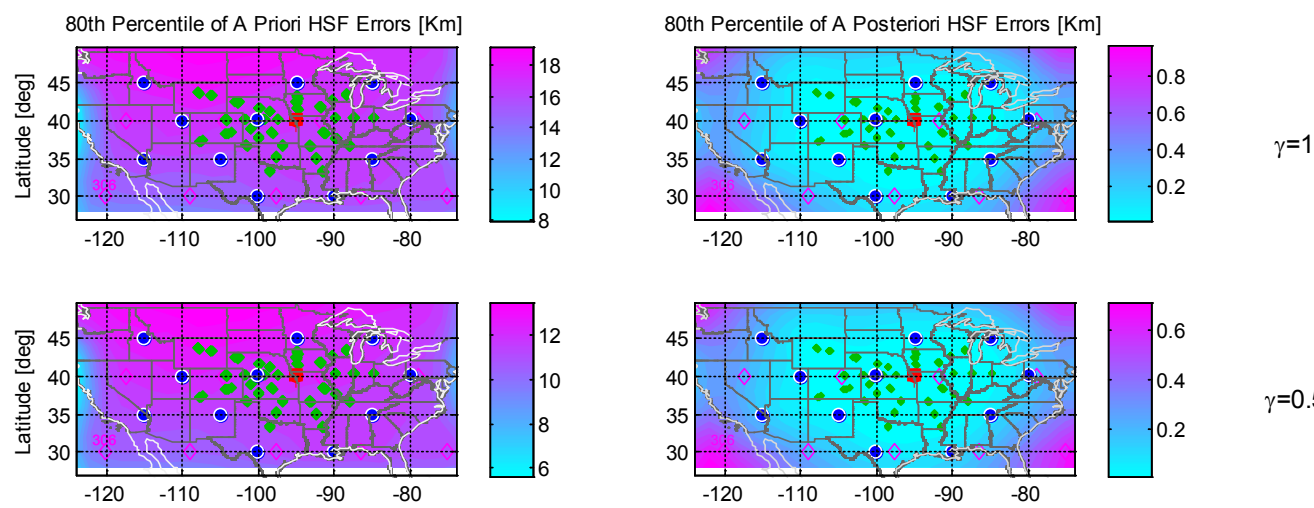

$\gamma=0.5$
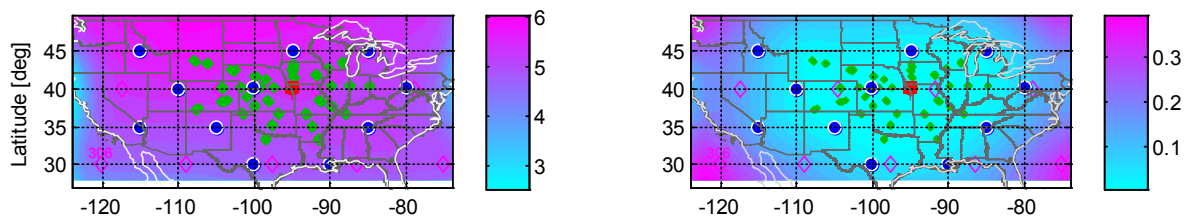

$\gamma=0.1$
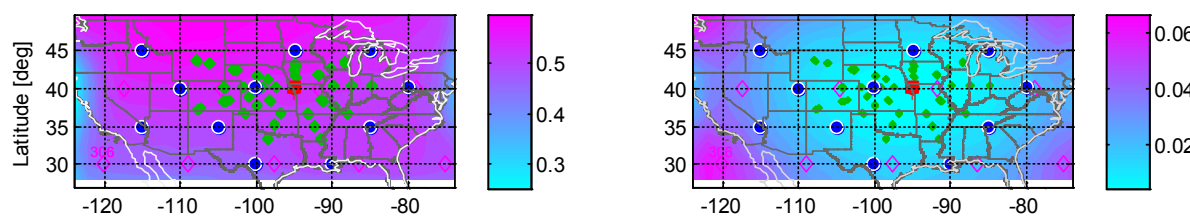

$\gamma=0.001$
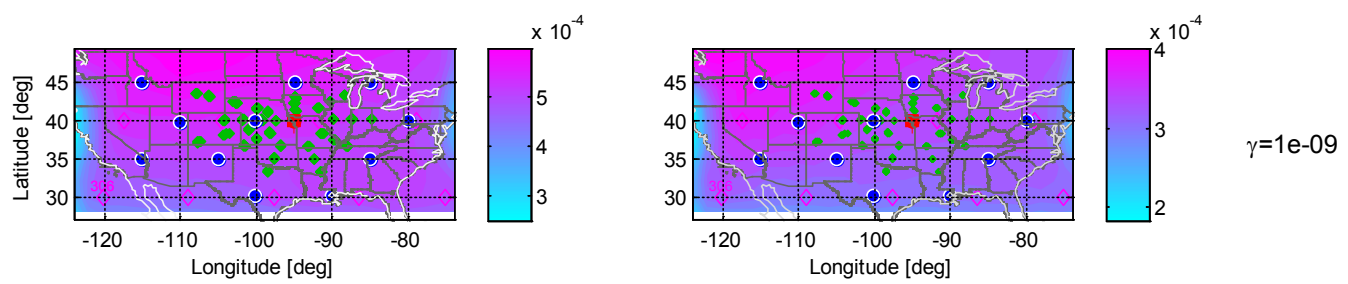

Figure 67: Eightieth percentile value maps for the a priori (left column) and a posteriori (right column) estimates' errors for the ionospheric scale height parameter $h_{\text {sf }}$ for Test Case H1. Each row corresponds to a different value of $\gamma$.

As with fixed-ionosphere-model test cases, a variation of the base test case is used to assess performance sensitivity to ground stations placement. Test Case H3 is characterized with a ground stations setup similar to that of Test Case E3, i.e., with an 
array of ground stations that are located along the US coastal and land borders. All other parameters are identical to those of Test Case $\mathrm{H} 0$ except for minor adjustments in signal frequencies that were necessary in order to maintain physical feasibility.
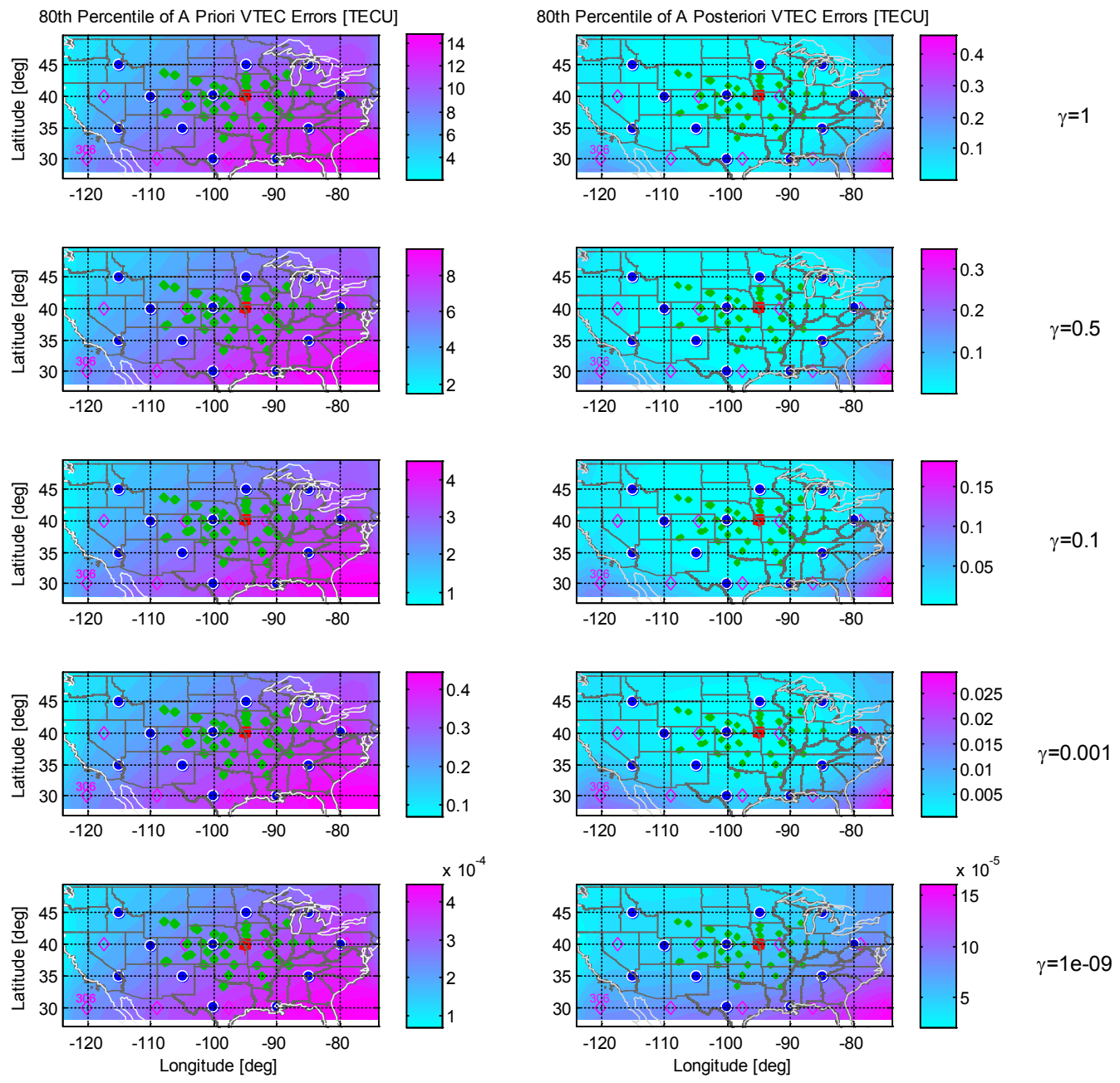

Figure 68: Eightieth percentile value maps for the a priori (left column) and a posteriori (right column) estimates' errors for the ionospheric vertical total electron content parameter $V T E C$ for Test Case H1. Each row corresponds to a different value of $\gamma$.

Figure 69 plots the horizontal and vertical 90\% error ellipses for Test Case H3. For this test case, the horizontal $90 \%$ error ellipse's semi-major axis is slightly longer than 
that of the base Test Case H0, while the ellipse's semi-minor axis is about twice as long as its Test Case H0 equivalent. Degradation in vertical accuracy by a factor of 6 has been observed as well. The horizontal $90 \%$ error ellipse that has been obtained for $\gamma=0.5$ covers the equivalent $90 \%$ error ellipse that has been computed for the fixed-ionospheremodel Test Case E3 (Fig. 51).

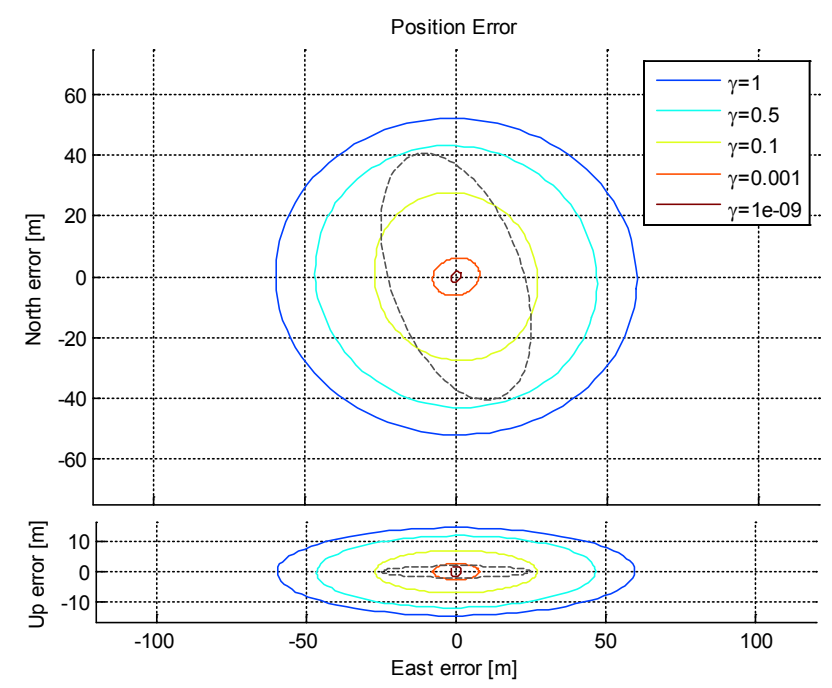

Figure 69: Horizontal and vertical 90\% error ellipses for Test Case H3 with different values of $\gamma$. The dashed ellipses in gray are the $90 \%$ error ellipses for Test Case H0 with $\gamma=1$.

Eightieth percentile error maps for the three Chapman parameters are shown in the next three figures. Residual errors for the peak electron density height $h_{\text {max }}$ parameter, shown in Fig. 70, appear comparable to those of Test Case H0 in the region that is above CONUS, with typical values below $1 \mathrm{~km}$. Some degradation has been observed for the

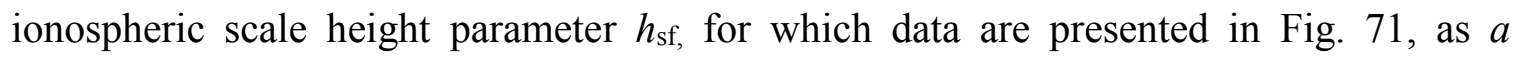
posteriori errors for this test case are roughly $40 \%$ increased in comparison to the base test case. Eightieth percentile error values for the ionospheric vertical total electron 
content parameter $V T E C$, whose maps are shown in Fig. 72 , are about $20 \%$ larger than with Test Case H0.
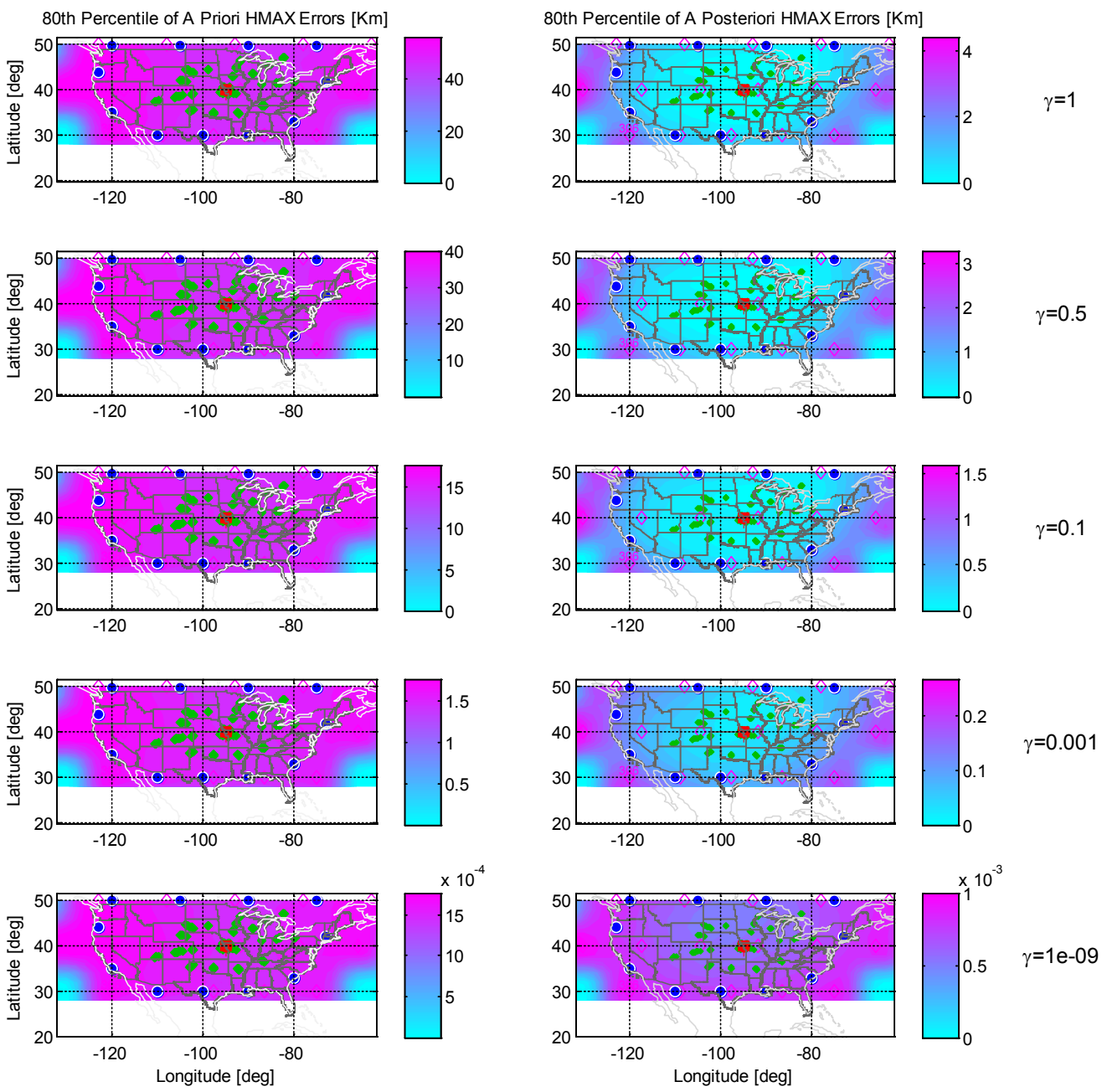

Figure 70: Eightieth percentile value maps for the a priori (left column) and a posteriori (right column) estimates' errors for the ionospheric peak electron density height parameter $h_{\max }$ for Test Case H3. Each row corresponds to a different value of $\gamma$. 

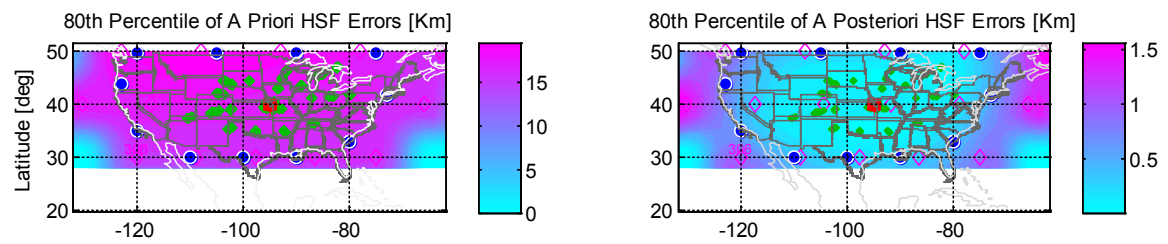

$\gamma=1$
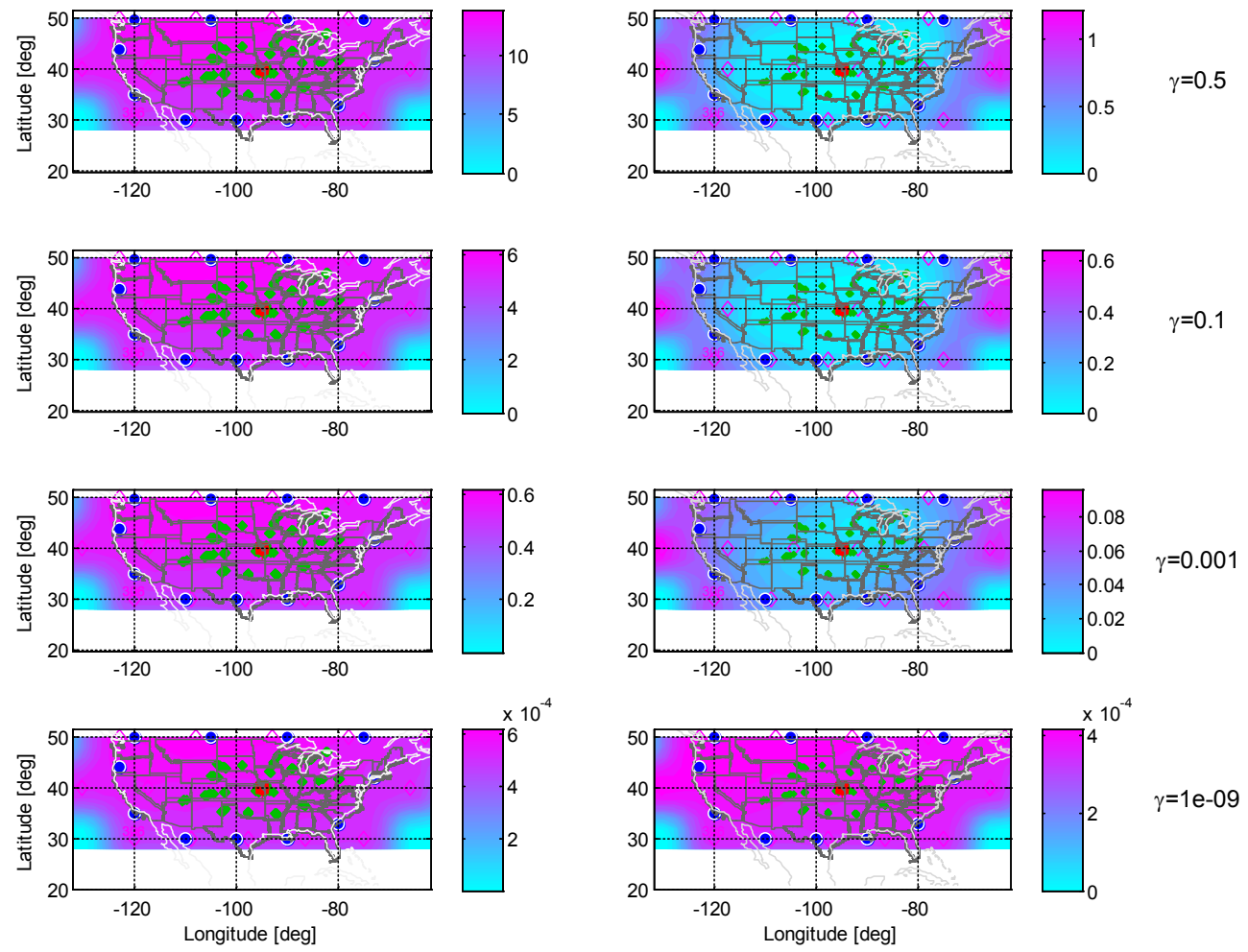

Figure 71: Eightieth percentile value maps for the a priori (left column) and a posteriori (right column) estimates' errors for the ionospheric scale height parameter $h_{\mathrm{sf}}$ for Test Case H3. Each row corresponds to a different value of $\gamma$. 

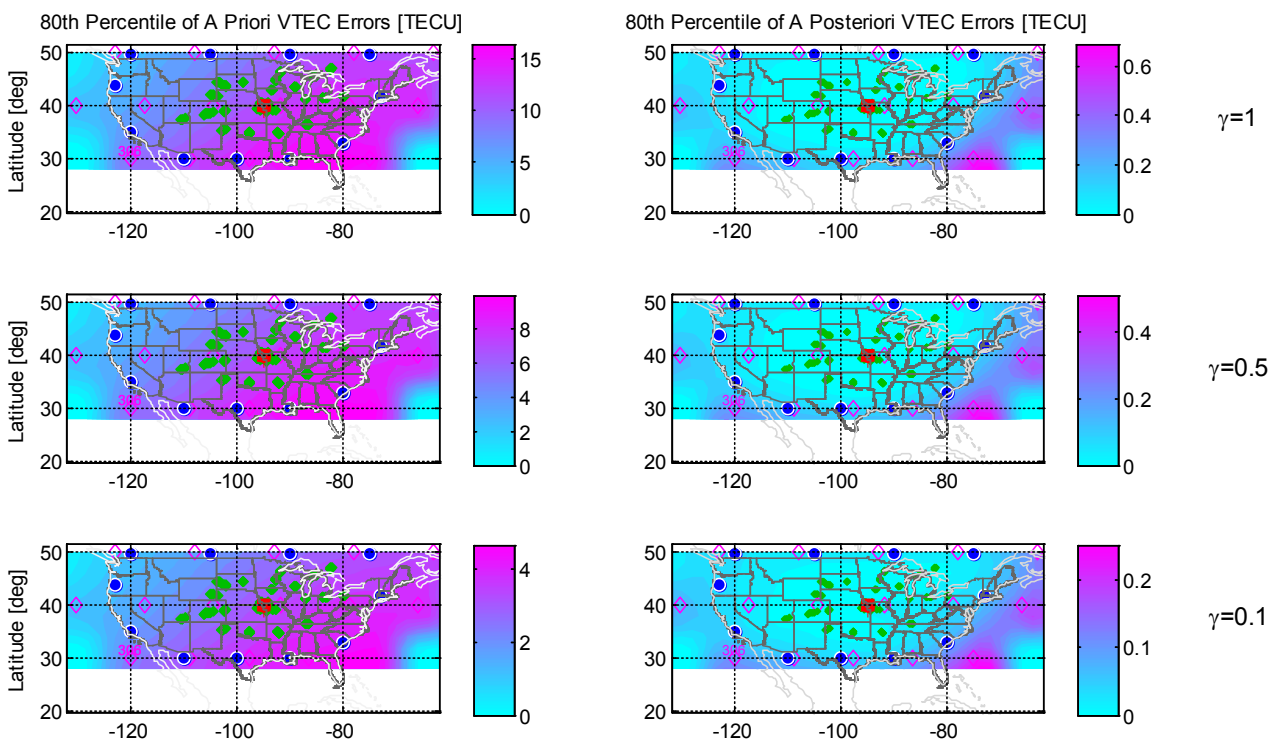

$\gamma=0.1$
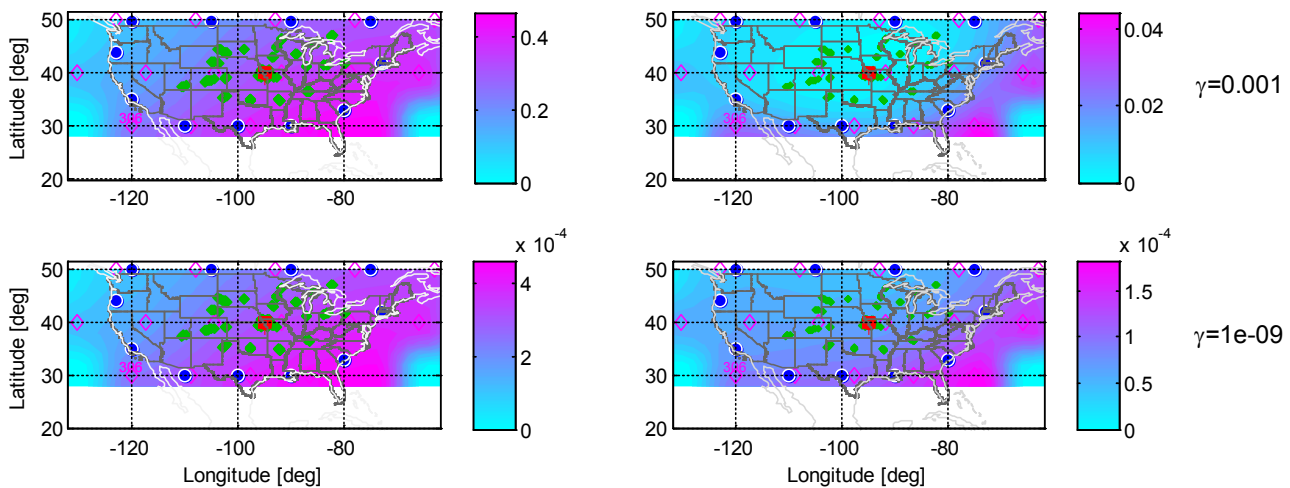

Figure 72: Eightieth percentile value maps for the a priori (left column) and a posteriori (right column) estimates' errors for the ionospheric vertical total electron content parameter VTEC for Test Case H3. Each row corresponds to a different value of $\gamma$.

The last test case studied in this group is Test Case H4, whose 1-sigma noise error was increased to 10 meters for the carrier phase measurements and kept unchanged at 1000 meters for the range-equivalent group delay pseudoranges. The significant degradation in positioning accuracy in comparison to the base Test Case H0 is well conveyed in Fig. 73 which shows the horizontal $90 \%$ error ellipses for both cases. 


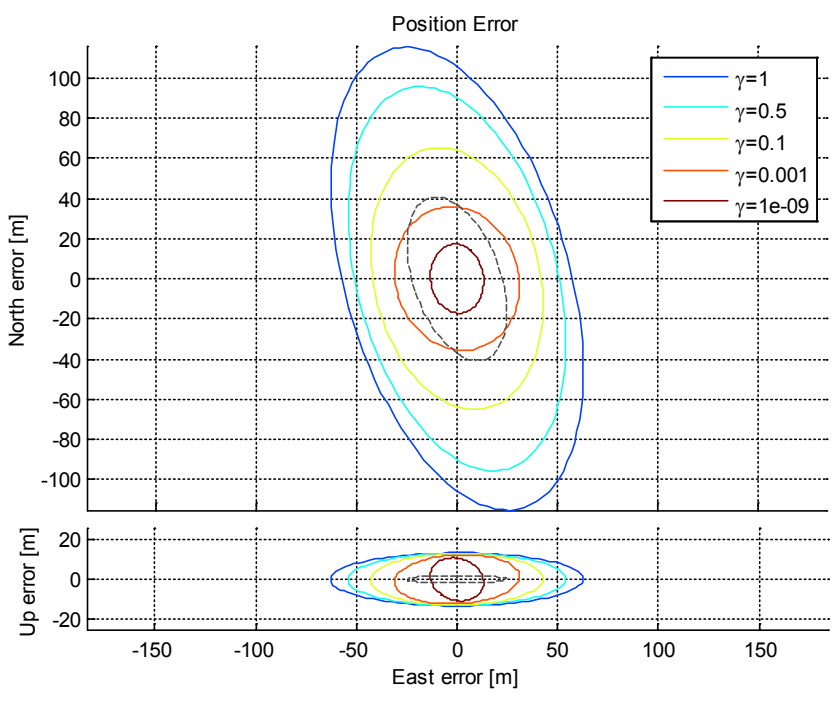

Figure 73: Horizontal and vertical 90\% error ellipses for Test Case H4 with different values of $\gamma$. The dashed ellipses in gray are the $90 \%$ error ellipses for Test Case H0 with $\gamma=1$. 


\subsubsection{Test cases with a medium number of available ray-paths (Group G)}

This subsection presents the performance for a scenario with a reduced number of available measurements. Test cases of Group G consider a set of ground stations that is a subset of the set of ground stations used with the high-availability-scenario test cases of Group H. Each ground station is assumed to transmit between 1 and 3 signals with varying frequencies, so that the total number of ray-paths received at the receiver is 17 and the total number of measurements processed, including both group delay and beat carrier phase measurements, is 136.

Test Case G0 is the base test case for this scenario with a medium number of available ray-paths. The HF signals for this test case have frequencies in the range 3.0-6.0 MHz. The number of hops for each ray-path is 1-4, with a mixture of signals arriving from above and from below at the UE.

The impact of utilizing significantly less measurements than in Test Case H0 is immediately evident (Fig. 74), as the 90\% error ellipse for Test Case G0 is larger by a factor of about 4 in both length and width for the case of $\gamma=1$. Comparing the results plotted in Fig. 74 with the results plotted in Fig. 54, it can be inferred that the horizontal $90 \%$ error ellipse computed for $\gamma=0.5$ will contain the horizontal $90 \%$ error ellipse of Test Case D0. However, note that the former ellipse is significantly larger, and the latter has a mean that is significantly further from zero. 


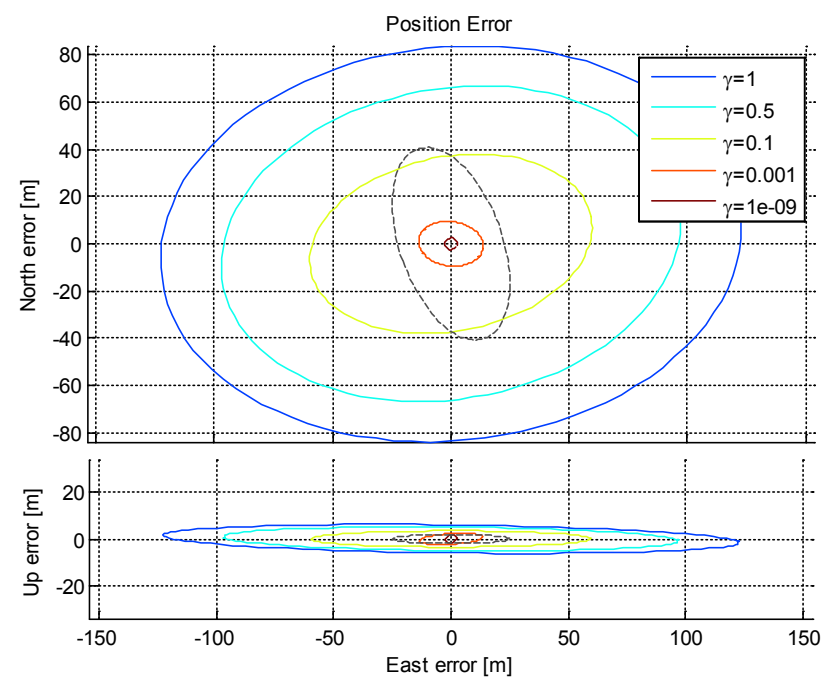

Figure 74: Horizontal and vertical 90\% error ellipses for Test Case G0 with different values of $\gamma$. The dashed ellipses in gray are the $90 \%$ error ellipses for Test Case H0 with $\gamma=1$.

Eightieth percentile error maps for the three Chapman parameters are shown in the next three figures. Consistent with the inferior positioning accuracy observed, the peak electron density height $h_{\max }$ parameter map, shown in Fig. 75, residual errors are about $25 \%$ bigger than those computed for the high measurement availability Test Case H0. Residual errors for the scale height parameter $h_{\text {sf }}$ parameter (Fig. 76) similarly exhibit degraded performance in the form of $20 \%$ larger residual errors. 

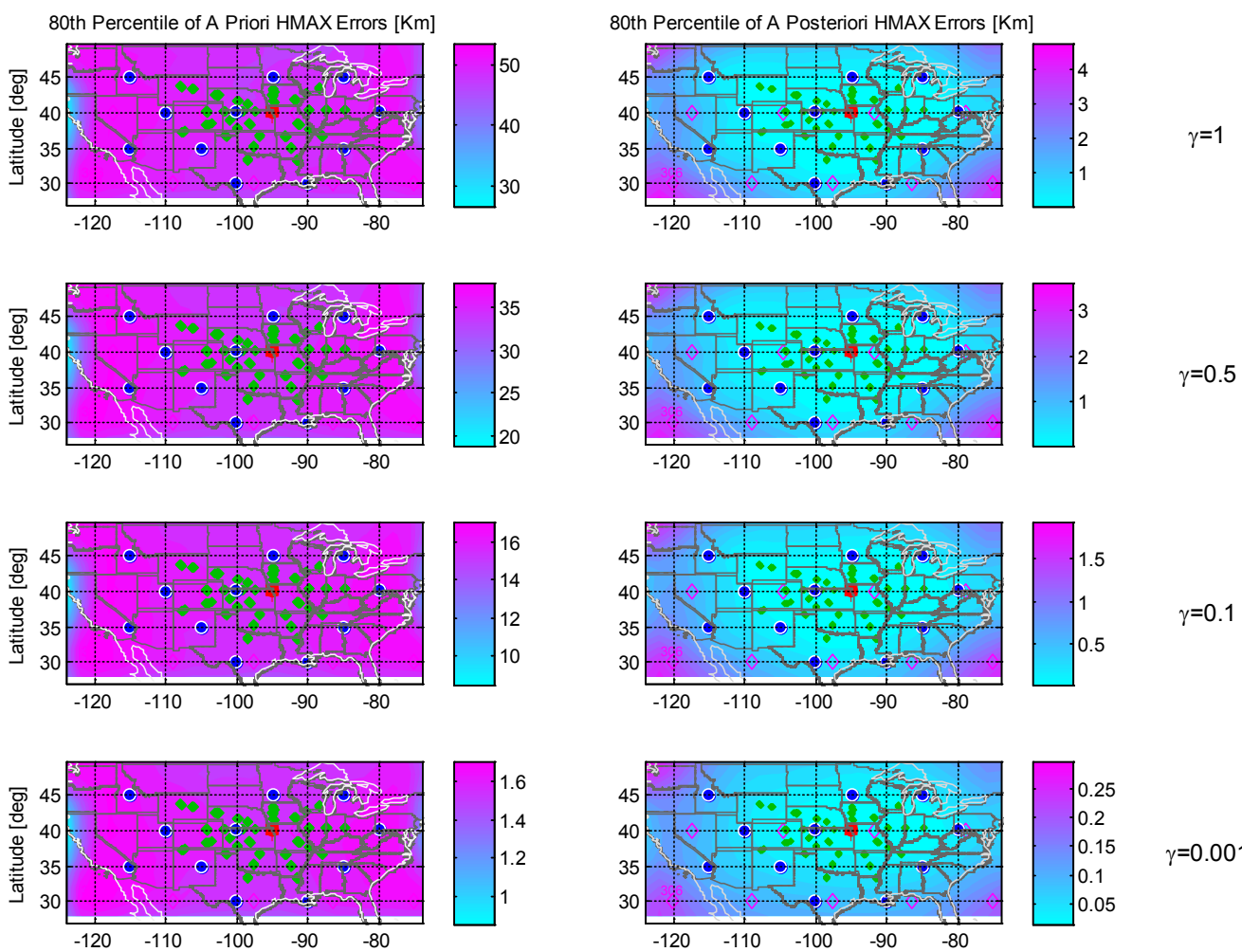

$\gamma=0.001$
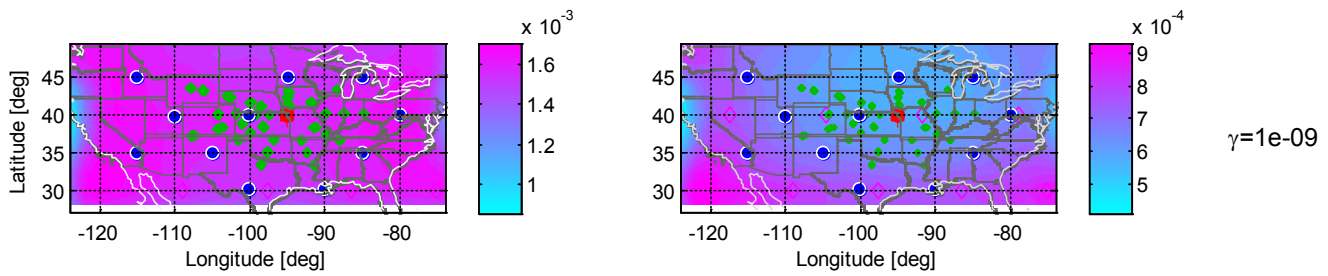

Figure 75: Eightieth percentile value maps for the a priori (left column) and a posteriori (right column) estimates' errors for the ionospheric peak electron density height parameter $h_{\max }$ for Test Case G0. Each row corresponds to a different value of $\gamma$. 

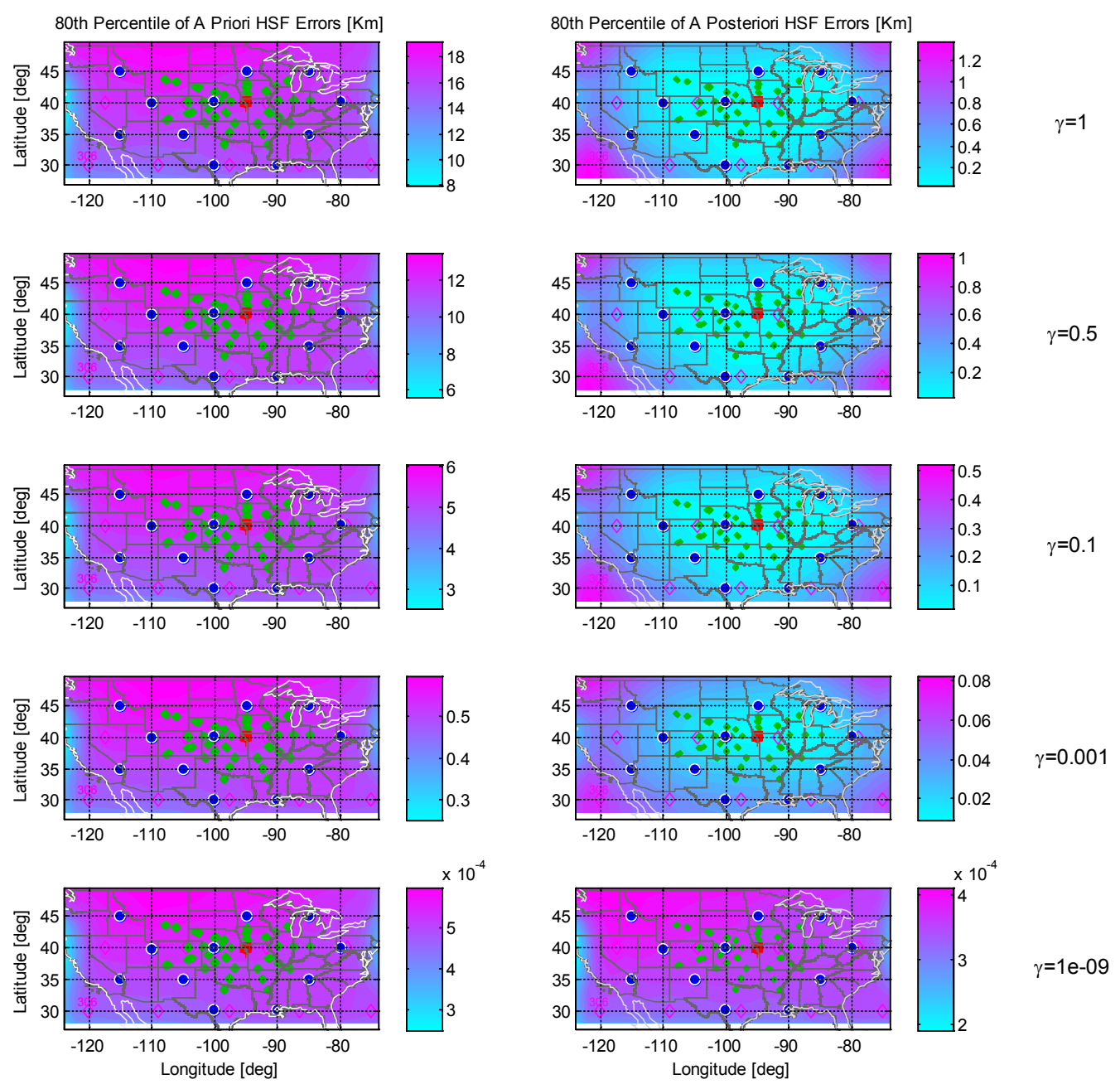

Figure 76: Eightieth percentile value maps for the a priori (left column) and a posteriori (right column) estimates' errors for the ionospheric scale height parameter $h_{\mathrm{sf}}$ for Test Case G0. Each row corresponds to a different value of $\gamma$.

For residual VTEC errors, shown in Fig. 77, some degradation from the results obtained for Test Cade H0 is evident. However, the relative errors reduction from the $a$ priori errors is significant. 

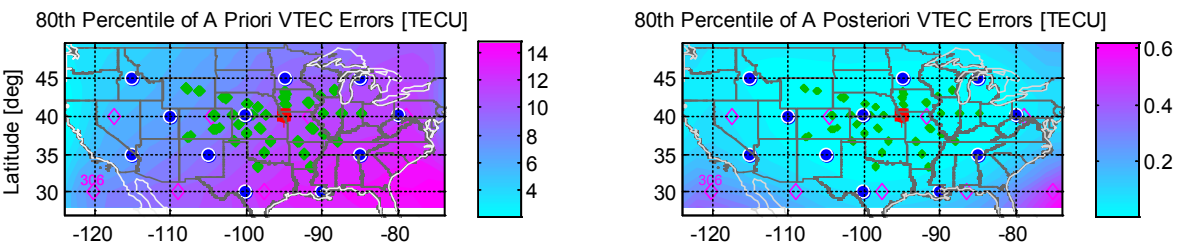

$\gamma=1$
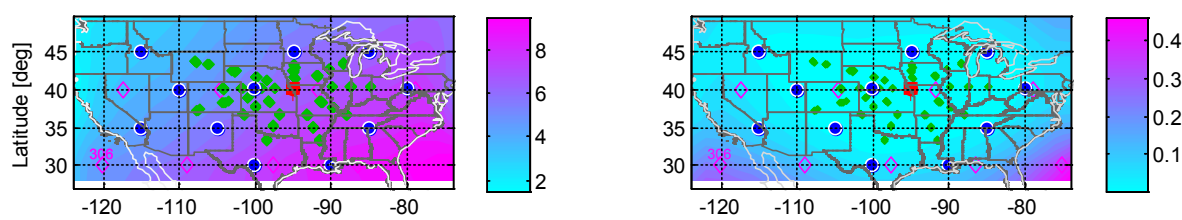

$\gamma=0.5$
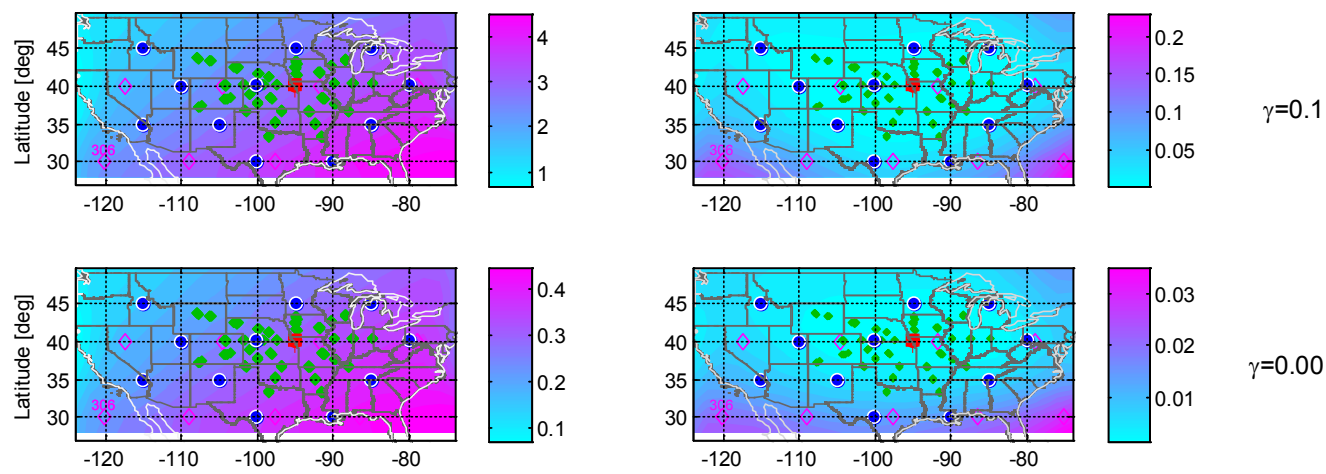

$\gamma=0.001$
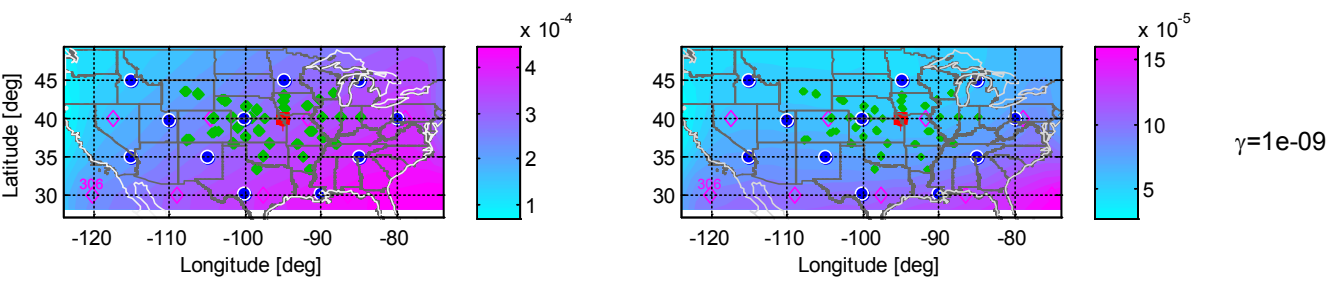

Figure 77: Eightieth percentile value maps for the a priori (left column) and a posteriori (right column) estimates' errors for the ionospheric vertical total electron content parameter VTEC for Test Case G0. Each row corresponds to a different value of $\gamma$. 


\section{CHAPTER 8}

\section{SYSTEM PERFORMANCE ANALYSIS, DISCUSSION,}

AND FUTURE DIRECTIONS

Results for the twenty one test cases presented in this study suggest that the problem is sufficiently observable to make this system a candidate for navigation in GNSS-denied situations. That is, a position solution can be obtained to a reasonable level of accuracy despite uncertainty about the ionosphere. At the same time, the filtered estimates of the ionosphere electron density profile parameters tend to have significantly reduced errors in comparison to their a priori estimates. Therefore, this method may also be useful for remote-sensing-based ionosphere characterization in cases where the receiver location is known a priori.

This chapter discusses the findings for the performance analysis of Chapter 7. The discussion is structured such that conclusions are drawn at the level of individual test cases, groups of test cases, or even classes. A significant part of this discussion will be dedicated to the manner in which the various configuration parameters influence system performance. This is done through highlighting apparent behavioral trends that have been observed with the various test case executions. 


\subsection{Positioning Characteristics}

Positioning accuracy for Group E's base test case, Test Case E0, is navigation grade, meaning that with a sufficient number of signals received and dual group-delay/beatcarrier-phase measurement processing, the achieved accuracy is adequate for the purpose of navigation and guidance with most applications.

As shown earlier, this test case has a fixed-ionosphere error model with an associated IEI value of -0.2276 . This value of the Ionosphere Error Index places it at the $65^{\text {th }}$ percentile, and therefore makes it reasonable to regard Test Case E0 as a 'typical' scenario under the assumption that the true uncertainty for the ionosphere can be reliably modeled by $M_{0}$. The fixed ionosphere model error induces a mean error of 5 meters. With a 25 meters horizontal 90\% errors ellipse semi-major axis, and a vertical error standard deviation of less than one meter, the obtained position solution can be used to determine the location of a vehicle as an independent source or as input to an integrated Inertial Navigation System (INS). The same conclusion holds for Test Case E1. Performance for other test cases of Group E may be adequate for navigation, depending on the application.

When a random error model for the ionosphere was considered in Test Case H0, with the same ground transmitters/ray-paths setup of Test Case E0, a similar level of accuracy has been observed for $\gamma$ values of near 0.1. Based on the plots of Fig. 61 of Test Case H0, it can be concluded that navigation grade positioning can be achieved with $\gamma$ values that are smaller than 0.2 . In other words, based on this analysis, navigation grade accuracy can be achieved if the true uncertainty for the ionosphere error model can be reliably modeled as $\gamma M_{0}$ where $\gamma<0.2$. 
With significantly fewer ray-paths used, Test Case G0's positioning accuracy has been proven inferior to that of Test Case H0. In this case, navigation grade accuracy with a $90 \%$ horizontal error ellipse semi-major axis of less than 25 meters can only be achieved when the ionospheric uncertainty can be reliably modeled with $\gamma$ values that are less than 0.01 . Vertical positioning, on the other hand, is very accurate for all values of $\gamma$. This may be significant for specific applications that are required to maintain position within a corridor that is limited in its altitude range.

An important result is the level of accuracy that can be obtained with the setup of Test Case H1, for which all signals approach the receiver from above. It has been observed that navigation grade accuracy can be achieved for very large values of $\gamma$. In fact, in spite of the relative degradation in vertical accuracy, positioning accuracy for this test case is navigation grade even with an extreme value of $\gamma=1$.

Class 3's Test Case F0 for which only group delay measurements are processed, exhibits characteristics that are unacceptable for navigation. Consistently, none of the fixed-ionosphere test cases of Classes 1 demonstrated navigation grade accuracy when realistic measurement noise standard deviation was considered. The test case in this class which exhibits greatest accuracy is Test Case $\mathrm{C} 0$ that is characterized by an ionosphereinduces mean horizontal error of 127 meters and a 733 meters semi-major axis. It should be concluded therefore that sufficient accuracy for navigation purpose cannot be achieved when group delay measurements are used exclusively. 


\subsubsection{Sensitivity to the available number of measurements}

As noted before, positioning performance for the setup of Test Case G0, that has fewer ray-paths than Test Case H0, is inferior to the performance of Test Case H0. Both semimajor and semi-minor axes of the horizontal $90 \%$ error ellipse for this test case are about three times as big as those of Test Case H0. This was not the case for extremely small values of $\gamma$, however. Comparing Fig. 61 for Test Case H0 and Fig. 74 for Test Case G0, it can be concluded that positioning accuracy for the two test cases is comparable for a small value for $\gamma$ of about 0.001 . Put differently, as the ionosphere model errors get smaller, performance becomes less dependent on the number of received ray-paths.

Consistent with the above findings, the impact of having different numbers of available measurements on positioning accuracy for fixed-ionosphere test cases is clear, as it can be concluded from Tables 2 and 5. Scenarios with low and medium numbers of available signals, as for the test cases of Groups A, B and D, exhibit notably inferior accuracy, evident in the dimensions of their horizontal $90 \%$ error ellipses, and, to some extent, in their vertical accuracy. With group delay processing only (Class 1 test cases), the base test case for the medium number of available signals, Test Case B0, has a $90 \%$ error ellipse that is 2.5 times bigger in length and twice as big in width as that of the base test case for the scenario with a high number of available signals, Test Case C0. Similarly for Class 2's test cases, the high number of measurements Test Case E0 yielded a 90\% horizontal error ellipse that is $1 / 3$ the length and $1 / 3$ the width of the ellipse that was computed for the case with a medium number of available measurements, base Test Case D0. 
Having a limited number of available measurements, therefore, sets a clear bound on positioning accuracy. It is clear that navigation grade accuracy cannot be achieved with only 17 ray-paths available that are sampled at four different frequencies, as with Group D's and G's test cases, unless the system is given a very good model for the ionosphere.

\subsubsection{Sensitivity to signals' vertical direction of arrival}

The signals' directional geometry as they arrive at the receiver has also been proven to impact accuracy. With fixed-ionosphere model test cases, when signals arrive at the receiver from above the horizon only, as in Test Cases B1 and E1, a significant increase in the vertical direction error by a factor of more than 5 was observed. However, with the dual group delay/carrier phase measurements processing of Class 2 test cases, even the somewhat degraded accuracy in the vertical direction is still adequate for navigation.

This observation is supported by the statistical analyses that have been performed for the random-ionosphere model Test Case H0 and Test Case H1. From the plot in Fig. 65 it is clear that while horizontal accuracy is improved by a factor of 2 for both axes of the horizontal 90\% error ellipse when all signals arrived from above, vertical accuracy degrades by a factor of about 8 . Even with the relatively degraded accuracy in the vertical direction, errors are expected to provide navigation grade accuracy.

This result may be relevant to antennas with limited Field Of View (FOV). It is also relevant to applications where the vehicle's fuselage is expected to block a significant portion of the antenna's FOV. 


\subsubsection{Sensitivity to a priori ionosphere model error}

While the statistical analysis of the test cases of Groups 3 and 4 cannot provide information for specific a priori ionospheric setups, the impact of the ionosphere model characteristics of specific setups can be studied through examination of fixed-ionosphere model test cases.

Equation (99) predicts that large initial errors in the a priori Chapman model parameters will result in a significant mean error for position and clock offset. Results for test cases B2 and E2 confirm that with a poor a priori ionosphere model, the positioning mean error (or positioning ionosphere-induced bias) is indeed further from zero than with the base test cases that have smaller initial $\xi$ values.

With the combined group-delay and beat-carrier-phase measurement processing of Class 2's test cases, where position errors are typically close to their means (i.e., where error ellipses are relatively small), the extent of degradation in the accuracy of the $a$ priori-ionosphere-error-induced mean position error will mostly determine whether the system is capable of providing the required level of accuracy for navigation. For Test Case E2 that is characterized by a very significant ionosphere models discrepancy, the mean horizontal position error of 15 meters is still acceptable for navigation with some applications. This is not true, however, with Test Case D2 for which the enlarged mean error is most likely too large for navigation.

For the random-ionosphere-model test cases of Classes 3 and 4, it is assumed that the actual ionosphere parameters' error covariance equals the above $M$ matrix that is considered with the batch filtering algorithm. That is to say, actual errors in the 
ionosphere model are distributed in a way that is consistent with their assumed distribution. With this in mind, the results shown in Fig. 57 for Test Case F0 demonstrate how position accuracy is closely related to the magnitude of the a priori errors in the ionosphere model. With a very good a priori model of the ionosphere, i.e., the case of $\gamma=10^{-9}$, position errors are primarily due to measurement noise. It is evident that as $\gamma$ increases, the horizontal position error increases, until it reaches a maximum where $\gamma=1$, for which the dimensions of the horizontal error ellipse are roughly doubled. Interestingly, the impact of increasing $\gamma$ on errors in the local vertical direction is very limited for this test case.

Similar observations have been made for other test cases of Classes 3 and 4. For Class 4's high-ray-paths-availability/combined-code-and-carrier-phase-ranging base test case, Test Case H0, however, the impact of increasing $\gamma$ has proven limited. Even in the worst case scenario of $\gamma=1$, the horizontal $90 \%$ error ellipse has a fairly small semi-major axis of 40 meters (Fig. 61). This is an important observation that suggests that the anticipated negative impact of very poor a priori ionosphere models may be alleviated when using the combined measurement types processing and given a sufficiently large number of available ray-paths.

With Test Case H1, where signals arrive at the receiver from above, increasing $\gamma$ results in a relatively significant increase in vertical position error (Fig. 65). For the case of $\gamma=1$ vertical errors are bounded by 15 meters. Although larger than those of Test Case $\mathrm{H} 0$, these vertical errors are still adequate for navigation. Similar results have been 
observed for Test Case $\mathrm{H} 3$, which is characterized by an alternative ground stations placement.

Figure 73, which shows position errors for Test Case H4, demonstrates how increasing $\gamma$ might not only increase the size of the $90 \%$ error ellipses, but also change their shape. For this test case, positioning becomes relatively more prone to errors in the south-north direction as $\gamma$ increases.

An important result concerns the combined group delay/beat carrier phase processing Test Case G0 that is characterized by limited ray-paths availability. As shown in Fig. 74, vertical accuracy remains very good even for the case of $\gamma=1$, for which vertical position errors are less than 7 meters. This observed property of the position error distribution suggests that the proposed system may be suitable for certain types of application.

\subsubsection{Sensitivity to ground stations placement}

Test Case B3 for Class 1, Test Case E3 for Class 2, and Test Case H3 for group 4 are considered in this subsection. These are the alterative-ground-station-placement variants from the base Test Cases of Groups B, E and H, in which ground stations are located along the land and sea borders of the United States.

For the fixed-ionosphere model test cases, i.e., Test Cases B3 and E3, it has been observed that ground station placement impacts positioning accuracy. However, results are somewhat inconclusive. The horizontal error ellipse for Test Case B3 is smaller than that of the base Test Case B0, yet Test Case E3's ellipse is about five times bigger than that of Group E's base test case, case E0. 
Comparing the results obtained for the random-ionosphere Test Case $\mathrm{H} 3$ with those of the base Test Case H0, it is evident that with the former, accuracy has degraded significantly both in the horizontal plane and in the vertical direction.

\subsubsection{Sensitivity to measurement noise covariance matrix and actual noise magnitude}

The measurement noise covariance matrix, $R$, that is used with the batch filter, affects both the a-priori-ionosphere-error-induced mean error and the way errors are distributed about their mean value. This result could be anticipated by inspecting both terms of the right hand side of Eq. (99). While $R$ enters the two right-hand-side terms through the $S_{l}^{-1}$ term (and additionally explicitly for the left term), the actual noise term, $\underline{v_{z}}$, affects only the left term of the equation, meaning that it only affects how errors are distributed about the mean error.

Looking at Figs. 28 and 43 that describe position errors for the fixed-ionosphere base Test Cases B0 and C0, and comparing them with Figs. 40 and 44 that plot errors for these test cases' reduced-measurement-noise variants, one can conclude that significantly different mean errors have been observed when scaled-down $R$ matrices were considered by the batch-filtering algorithm. This result is consistent with the observations of the previous paragraph.

The manner in which measurement error standard deviation affects positioning accuracy can be studied through a comparative investigation of the $90 \%$ error ellipses that have been obtained for test cases B0 and B4, the $90 \%$ error ellipses that have been obtained for test cases $\mathrm{C} 0$ and $\mathrm{C} 4$, and the $90 \%$ error ellipses that have been obtained for 
test cases $\mathrm{H} 0$ and $\mathrm{H} 4$. Each of these pairs of test cases consists of a base test case and an altered-measurement-noise variant. Test Case B4's horizontal 90\% error ellipse has semimajor and semi-minor axes that are about 16 and 21 times smaller than those of Test Case B0's semi-major and semi-minor axes, respectively. Similarly, Test Case C4's horizontal $90 \%$ error ellipse has semi-major and semi-minor axes that are roughly 25 and 48 times smaller than those of Test Case C0's semi-major and semi-minor axis, respectively. Accuracy degradation for Test Case $\mathrm{H} 4$ is somewhat moderate in terms of changes in the dimensions of the $90 \%$ error ellipses in comparison to Test Case H0. For an increase by a factor of 10 for the presumed beat carrier phase measurement noise, an increase by a factor of 3 has been observed for the dimensions of the $90 \%$ error ellipse's semi-major and semi-minor axis, which is primarily oriented in the north-south direction -- compare Figs. 61 and 73. The minor axis of the north-south/east-west ellipse and the vertical error extent, however, show larger increase with the increased beat carrier phase measurement error of Test Case H4.

These comparisons lead to the somewhat straightforward conclusion that both the magnitude of the mean error (to some extent) and the error distribution about that mean (in terms of the $90 \%$ error ellipses) are affected by the magnitude of the considered measurement noise. 


\subsubsection{Sensitivity to additional parameters}

The beneficial impact of having a wide range of signal frequencies on positioning accuracy was also evident when variations of the test cases shown here were tested with a limited range of signal frequencies. These characteristics of the method have been observed in many sub-cases that have not been presented in this dissertation for the sake of brevity. 


\subsection{A Posteriori Ionosphere Model Accuracy}

The algorithm has proven successful in reducing errors for the a priori ionosphere model parameters in all studied test cases. As one might expect, smaller errors in terms of the latitude/longitude dependent $80^{\text {th }}$ percentile have been observed near the areas where ray-paths travel through the ionosphere. These regions where electron density is probed can be identified in the different a priori / a posteriori Chapman-parameters-estimates plots by the green points that designate ground bounce off points, as in Fig. 25 and all similar figures that plot ionosphere parameter errors using a color contour map.

\subsubsection{Scenarios with a High Number of Available Signals}

For the Class 1 scenario's base test case with a high number of signals, Test Case C0, the $80^{\text {th }}$ percentile CONUS area mean a posteriori error of the parameter $h_{\max }$ was $1.3 \mathrm{~km}$ and the mean a posteriori error for the parameter $h_{\mathrm{sf}}$ was $0.87 \mathrm{~km}$. At the same time, the mean a posteriori error for the parameter VTEC was $0.11 \mathrm{TECU}$. Smaller residual errors were obtained for the Class 2 scenario's base test case with a high number of signals, Test Case E0. Its CONUS area $80^{\text {th }}$ percentile mean a posteriori error for the parameter $h_{\max }$ was $0.42 \mathrm{~km}$ and the mean a posteriori error for the parameter $h_{\mathrm{sf}}$ was $0.30 \mathrm{~km}$. The mean a posteriori error for the parameter VTEC was as small as 0.04 TECU. Clearly, these values, roughly three times smaller than their Class 1 equivalents, benefit from the accuracy of the carrier phase measurements.

Additionally for Class 2's test cases, worse results in terms of Chapman parameters residual errors were obtained for the large a priori $\xi$ test case - Test Case E2. These errors, typically on the order of $0.1 \mathrm{~km}$ for $h_{\max }$ and $h_{\mathrm{sf}}$ and $0.1 \mathrm{TECU}$ for $V T E C$, are still 
at a level that makes the output of the dual measurements process a useful tool in ionosphere characterization.

In contrast, the smallest estimation errors were obtained for Test Case E1, where signals arrive at the receiver from above only. In fact, errors for this case are about $50 \%$ smaller than their Test Case E0's equivalents. The mean $80^{\text {th }}$ CONUS area percentile $a$ posteriori error for the VTEC parameter was 0.02 TECU.

For all fixed-ionosphere model test cases in this category, the final value for the $\xi$ parameter that was computed at the end of the optimization process was smaller than its initial value. It should be noted that since the $\xi$ parameter is defined in terms of the base 10 logarithm of normalized errors in $\underline{p}$, and since the elements of $\underline{p}$ are defined as natural logarithms of the Chapman parameters and their partial derivatives, changes in $\xi$ may not appear as dramatic as the observed reduction in Chapman parameters errors.

With random-ionosphere model test cases, performance is evaluated through determination of an upper bound for the a posteriori error's $80^{\text {th }}$ percentile in the region that is above the CONUS. Test case $\mathrm{H} 0$ a priori error's $80^{\text {th }}$ percentile for the ionosphere peak electron density height parameter $h_{\max }$ take values between 45 and 50 TECU when $\gamma=1$. A dramatic reduction in errors yields an upper bound of about 1 TECU for the corresponding a posteriori errors map. Consistent results are apparent for the cases of $\gamma=0.5, \gamma=0.1, \gamma=0.001$ and $\gamma=10^{-9}$. A significant error reduction has been observed for both the ionospheric scale height parameter $h_{\text {sf }}$ for which an a priori upper bound of about $18 \mathrm{~km}$ has been reduced to $0.5 \mathrm{~km}$. Similarly for the ionospheric vertical total 
electron density content parameter VTEC, an upper bound of 13 TECU for the a priori estimates has been reduced to less than 0.2 TECU for the a posteriori estimates.

The three Chapman parameters errors $80^{\text {th }}$ percentile maps for Test Case H1 exhibit results that are similar to those of the base test case. For the $h_{\text {sf }}$ parameter and for the VTEC parameter, residual $80^{\text {th }}$ percentile values which are roughly $15 \%$ lower than those of Test Case H0 were recorded. This result is qualitatively consistent with the result shown above for the equivalent fixed-ionosphere model test cases with signals arrive from above the horizon only.

The alternative ground stations placement along the land and sea borders of the CONUS has been shown to provide slightly inferior performance for Class 4's Test Case $\mathrm{H} 3$ in terms of applying corrections to the ionospheric model. With the $h_{\max }$ parameter $80^{\text {th }}$ percentile maps, the a posteriori estimation errors' upper bounds for the different values of $\gamma$ are roughly $30 \%-50 \%$ larger than their Test Case $\mathrm{H} 0$ equivalents. This is also the case for the $h_{\text {sf }}$ parameter, and to a lesser extent, for the VTEC parameter. It should be noted, however, that even with the relative degradation in performance that has been observed, the magnitude of reduction in Chapman parameter errors from their corresponding a priori values is still very significant. For the VTEC parameter, for instance, an upper bound for the a priori $80^{\text {th }}$ percentile error map of 16 TECU has been reduced to about 0.2 TECU. It can be concluded, therefore, that the wider-spread array of ground stations of Test Case $\mathrm{H} 3$ seemed to have performed only slightly worse than that of the base Test Case H0. 


\subsubsection{Scenarios with Medium and Low Numbers of Available Signals}

Analyzing Class 2 test cases, it can be concluded that reducing the number of available measurements affected mostly the observability of the Chapman $h_{\max }$ parameter, for which residual a posteriori errors are about twice as big for test cases D0 and D2 of Group D in comparison to their Group E equivalents - Test Cases E0 and E2. Still, $a$ posteriori residual errors for this parameter are less than one tenth of their a priori values. A posteriori errors for the $h_{s f}$ and VTEC parameters remained fairly small (Table 6), with a mean $80^{\text {th }}$ CONUS area percentile value of 0.05 TECU for the base Test Case D0 and a mean $80^{\text {th }}$ percentile value of 0.10 TECU for the high-IEI Test Case D2. This suggests that for the purpose of ionosphere characterization, a relatively small number of received signals may be sufficient.

Similarly for group-delay-measurements-only test cases, comparing the medium number of available measurements Test Cases B0 and B4 with the equivalent high number of available measurements Test Cases $\mathrm{C} 0$ and $\mathrm{C} 4$, some degradation in performance has been noted for the test cases of Group B. In particular, residual errors for the $h_{\text {max }}$ parameter increased by a whole order of magnitude for Test Case B4. With even fewer ray-paths considered as with Test case A0, however, no further degradation in performance has been recorded.

Remarkably for Class 1 test cases, medium and low numbers of available measurements base test cases B0 and A0 exhibit only slightly worse results than the equivalent Class 2 base test cases. For instance, for Test Case A0 with 21 ray-paths, the

mean $80^{\text {th }}$ CONUS area percentile residual a posteriori error was 0.14 TECU. However, 
performance appears considerably more sensitive to initial IEI. Therefore, the $a$ posteriori errors for Test Case B2 are roughly five times bigger than for Test Case B0.

Two random-ionosphere model test cases were considered that differ in their number of available measurements. Inferior accuracy for all three Chapman parameters is evident for the medium number of available measurements test case, G0, in comparison with the high number of available measurements test case, H0. With Test Case H0, for the $h_{\max }$ parameter, the $80^{\text {th }}$ percentile errors are bounded by $1.5 \mathrm{~km}$ and $0.8 \mathrm{~km}$ for $\gamma=1$ and $\gamma=0.5$, respectively, whereas with Test Case G0, the bounds are $2 \mathrm{~km} 1.5 \mathrm{~km}$ for the same values of $\gamma$. For the $h_{\text {sf }}$ parameter and the same values of $\gamma$, bound values increased from $0.5 \mathrm{~km}$ and $0.4 \mathrm{~km}$ to $0.8 \mathrm{~km}$ and $0.9 \mathrm{~km}$, respectively. For the $V T E C$ parameter and the same values of $\gamma$, bound values increased from 0.25 TECU and 0.15 TECU to 0.35 TECU and 0.12 TECU, respectively. 


\subsection{Batch-Filtering Algorithm Functionality}

For the vast majority of cases, solutions for the minimization problem converged to what appears to be their global minimum. Thus, the presence of nonlinearities in the model does not pose a significant challenge to solving the underlying batch estimation problem. It appears that the occurrence of convergence to a solution that is different from the optimal solution was never encountered for any of the many thousands of simulation cases carried out throughout this study. This is evident in the fact that the batch-filtering algorithm has never driven the position solution to a solution that is different from the solutions that have been computed using the linear approximation of Eq. (97).

Some test cases failed to converge, as in Subsection 6.3.1. A further investigation of such cases showed that, in almost all of them, the cause was one of the two potential weaknesses that originate from bounce-point equation and ray-hop solution ambiguities, discussed in Subsection 6.3.2. For only a handful of simulation executions, the author was unable to determine the cause for what appears to be a failure in driving the solution to its optimal set of values.

The benefit of allowing selective use of available measurement is also evident. Many batch filter executions started by considering only a small subset of the available measurements in the early Gauss Newton iterations, but ended up using all measurements for later iterations as the algorithm drove the solution closer to its optimal set of values. This sort of behavior, typical for test cases with significant errors in their a priori ionospheric model, usually resulted in longer batch filter execution run times. 
A practical result concerns the importance of processing both group delay and beat carrier phase measurements. While the contribution of the latter to positioning accuracy has been widely discussed in this chapter, it has been observed that the use of pseudorange measurements is crucial for the robustness of the iterative process when estimated position and ionosphere parameters are still far from their optimal values. In fact, in most cases the solution is doomed to diverge unless pseudorange measurements are incorporated and processed in the early steps of the Gauss-Newton algorithm.

\subsection{Future Research}

An extension is planned for this study that supplements truth-model simulation tests with tests involving actual data from a network of HF beacons and receivers. Such a network is being deployed in South America [38]. It is reasonable to assume that the problem that will be addressed with the true data experiment will somewhat differ from the fundamental problem that was studied in this work due to limited availability of received signals. This may include the case where receiver location and receiver clock error are known a priori, so that the problem is essentially defined as an ionosphere estimation problem.

A second extension should consider enhanced ionosphere modeling. As already mentioned, the current ionosphere parameterization does not allow modeling of distinct $\mathrm{D}$ and $\mathrm{E}$ layers. A greater physical fidelity will be achievable by incorporating increased complexity in the ionospheric model parameterization. Therefore, one useful extension for this work would be to employ a more realistic ionosphere model that would enable 
representation of the $\mathrm{D}$ and $\mathrm{E}$ layers. On the disadvantage side, an increased complexity of the 3-dimensional $N_{e}(\underline{r})$ distribution will increase the number of estimated ionosphere parameters that will be needed in order to characterize $N_{e}(\underline{r})$. This increased number of parameters will complicate the filter task of simultaneously estimating receiver position, receiver clock offset, and ionosphere parameter corrections.

A useful variant of this work would be to consider the case where the estimator lacks $a$ priori knowledge of the number of bounces for any given signal. This is the case where $m$ is unknown and must be estimated for each received signal while estimating the receiver position and clock error along with the ionosphere corrections. Such an estimator would solve a mixed real/integer batch filtering problem. The navigation community already has experience with such problems because they are used for precise Carrier-Phase Differential GPS (CDGPS) [39].

Another possible extension would be to augment the estimation problem with additional types of fused data. For example, ionogram data or GPS slant TEC data from a network of receivers might help to improve the estimates of the ionosphere model corrections. A different approach might be to use data obtained from a space weather data assimilation system such as GAIM, as described in Ref. [40]. Any improvements to the ability to estimate the ionosphere or to characterize it a priori should also improve the receiver position and clock offset estimates. Of course, such a system would need a method of communicating the independent ionosphere data to the user receiver, which would complicate its infrastructure. 
Finally, a study that considers the case of a known receiver location is expected to be beneficial to the field of ionosphere remote sensing. It has been observed that elimination of the uncertainty that is associated with the receiver location results in enhanced accuracy for the unknown ionosphere parameters. 


\section{CHAPTER 9}

\section{SUMMARY AND CONCLUSION}

A batch filter algorithm has been developed that utilizes group-delay/pseudorange and beat carrier phase measurements from HF signals propagating in the ionosphere to solve a combined positioning/ionosphere-corrections problem. These HF signals are transmitted from stationary ground-based beacons at known locations. They propagate to an over-the-horizon user receiver at an unknown location via multiple bounces off of the ionosphere and the Earth.

The navigation filter estimates user position, user clock error, beat carrier phase measurement bias and corrections to parameters that characterize the ionosphere's threedimensional electron density profile. The latter parameters consist of information that is required to generate latitude/longitude dependent maps for three Chapman model parameters: height of the peak electron density, altitude scale factor, and vertical total electron content. As previously mentioned, a more realistic model would likely be needed for working with real data. The filter starts with a priori estimates of its parameter maps that are based on fits to the IRI model. It estimates corrections to these parameter maps as part of its navigation solution.

The nonlinear batch least-squares estimation problem is solved using a modified Gauss-Newton method. This method has a high rate of achieving successful convergence 
to the optimal value of the underlying cost function, despite the challenge of physical infeasibility of some signal ray paths at intermediate guesses of the problem solution.

System performance has been investigated using a truth-model simulation. The simulation and the corresponding batch-filter use an advanced model of HF signals that propagate in the ionosphere. Twenty one simulated test cases that consider various combinations of parameters' characteristics have been considered. Limited Monte-Carlo simulations have been performed to investigate the performance of the navigation filter. Theoretical covariance analysis has also been performed to investigate its performance.

The Monte-Carlo simulation results and the covariance analysis results indicate feasibility for the combined HF navigation/ionosphere-correction concept. It has been shown that with sufficient availability of received signals, navigation grade accuracy for positioning, where the $90 \%$ horizontal error ellipse's semi-major and semi-minor axes are both less than 20 meters and the vertical $90 \%$ error bound is less than 5 meters, may be achievable. A posteriori ionosphere models are consistently improved for these cases in comparison to their a priori counterparts. Computed a posteriori error $80^{\text {th }}$ percentiles upper bounds for a representative random-ionosphere model test case were less than 0.75 $\mathrm{km}$ for the $h_{\max }$ parameter, $0.35 \mathrm{~km}$ for the $h_{\mathrm{sf}}$ parameter, and 0.1 TECU for the VTEC parameter, whereas the corresponding a priori upper bounds were $30 \mathrm{~km}$ for $h_{\max }, 10 \mathrm{~km}$ for $h_{\mathrm{sf}}$, and 5 TECU for $V T E C$.

The important contribution of beat carrier phase measurements to position solution accuracy is clearly evident. Beat carrier phase is useable by the navigation filter if a given signal path from a transmitter to the receiver carries signals with a continuously varying 
succession of different frequencies so that the common-mode carrier phase bias can be estimated and effectively removed from the problem. For a representative test case that relies on group delay measurements only, the computed $90 \%$ horizontal error ellipse's semi-major and semi-minor axes were 100 meters and 70 meters, respectively. When beat carrier phase measurements were added to the batch-filter, the $90 \%$ horizontal error ellipse's semi-major and semi-minor axes decreased to 35 meters and 20 meters, respectively.

It has been demonstrated how performance is affected by various characteristics of the estimation problem. Positioning accuracy is influenced by the level of uncertainty of the a priori ionosphere model, by the number of available measurements, and receiver ranging error standard deviation, both group-delay errors and beat carrier phase errors. A decrease in the a priori ionosphere modeling error tends to decrease the horizontal and vertical position error. A decreased number of available measurements, however, will have the opposite effect on positioning accuracy, although this trend becomes less distinct as the a priori ionosphere modeling errors become smaller. The magnitude of the ranging error standard deviation has been shown to directly impact the size of the horizontal and vertical position $90 \%$ error ellipses, with a relatively moderate impact for the random-ionosphere-model test cases compared to the fixed-ionosphere-model test case. With test cases where signals approach the receiver from above, an enhanced horizontal accuracy has been observed while vertical position accuracy has degraded. Inconclusive results have been obtained for an attempt to assess the impact of ground stations placement on positioning performance. 


\section{REFERENCES}

[1] X. Huang and B. W. Reinisch, "Real-Time HF Ray Tracing through a tilted Ionosphere," Radio Science, vol. 41, 2006.

[2] N. Y. Zaalov, E. V. Moskaleva and T. S. Burmakina, "Application of the IRI model to the HF propagation modelwith optimization of the ionosphere parameters to day-to-day variation," Advances in Space Reasearch, vol. 60, no. 10, 2017.

[3] A. S. Juras, Handbook of geophysics and the space environment, Hanscom AFB, MA: Final Report Air Force Geophysics Lab, 1985.

[4] P. Misra and P. Enge, Global Positioning System, Ganga-Jamuna Press, 2011.

[5] Y. Baumgarten and M. L. Psiaki, "Navigation Using High-Frequency Ground Beacons and Ionosphere Model Corrections," Navigation, vol. 64, pp. 255-271, 2017.

[6] A. Ishimaru, Electromagnetic Wave Propagation, Radiation, And Scattering, Seatle, WA: IEEE Press, 2017.

[7] S. A. Cummer, "Modeling electromagnetic propagation in the Earth-ionosphere waveguide," IEEE Transactions on Antennas and Propagation, vol. 48, no. 9, pp. 1420-1429, 2000.

[8] D. L. Hysell, Antennas and Radar for Environmental Scientists and Engineers, Cambridge University Press, 2018.

[9] E. V. Appleton and A. F. Barnett, "Local Reflection of Wireless Waves from the Upper Atmosphere," Nature, pp. 333-340, 1925.

[10] I. B. Bernstein, "Geometric optics in space- and time-varying plasmas," The Physics of Fluids, vol. 18, no. 320, 1975. 
[11] J. S. Seybold, Introduction to RF Propagation, Hoboken NJ: Wiley, 2005.

[12] M. R. Jones and J. J. Stephenson, "A Versatile Three-Dimensional Ray Tracing Computer Program for Radio Waves in the Ionosphere," U.S. Dept. of Commerce, 1975.

[13] M. J. Lighthill, "Group Velocity," in Hyperbolic Equations and Waves., Berlin, Springer, 1965, pp. 96-123.

[14] J. Haselgrove, "Report of conference on the physics of the ionosphere," London Physical Society, London, 1954.

[15] K. G. Budden, Radio waves in the ionosphere; the mathematical theory of the reflection of radio waves from stratified ionized layers, Cambridge: University Press, 1961.

[16] M. L. Psiaki, "Ionosphere Ray-Tracing of RF Signals and Solution Sensitivities to Model Parameters," in The 29th International Technical Meeting of The Satellite Division of the Institute of Navigation, Portland OR, 2016.

[17] K. C. Yeh and C. H. Liu, Theory of Ionospheric Waves, New York, NY: Academic Press, 1972.

[18] N. G.-I. Agency, "World Geodetic System 1984 (WGS 84)," [Online]. Available:

https://web.archive.org/web/20120402143802/https://www1.nga.mil/ProductsSe rvices/GeodesyandGeophysics/WorldGeodeticSystem/Pages/default.aspx. [Accessed 15 May 2018].

[19] B. Hofmann-Wellenhof, H. Lichtenegger and J. Collins, Global Positioning system: Theory and Practice, Springer Wien New York, 2001, pp. 279-282.

[20] "U.S. Geological Survey, Department of the Interior, Data Catalog," USGS, [Online].

Available: https://catalog.data.gov/organization/usgs-gov. [Accessed April 2018].

[21] "International Geomagnetic Reference Field: 11th Generation," NGDC, [Online]. Available: https://www.ngdc.noaa.gov/IAGA/vmod/IGRF11_announce.pdf. [Accessed March 2017]. 
[22] M. L. Psiaki, K. C. Ward and K. J. DeMars, "A Bi-Quintic Latitude/Longitude Spline and Lunar Surface Modeling for Spacecraft Navigation," in Proc. Junkins Dynamical Systems Symposium, College Station, TX, 2018.

[23] D. Bilitza, "International reference ionosphere 2000," Radio Science, vol. 36, no. 2, pp. 261-275, 2001.

[24] B. W. Reinisch and D. Bilitza, "Karl Rawer's life and the history of IRI," Advances in Space Research, vol. 34, no. 9, pp. 1845-1850, 2004.

[25] D. Bilitza, "The international reference ionosphere today and in the future," Journal of Geodesy, vol. 85, no. 12, pp. 909-920, 2011.

[26] M. L. Psiaki, G. S. Bust and C. N. Mitchell, "Nonlinear Estimation to Assimilate GPS TEC Data into a Regional Ionosphere Model," in The 28th International Technical Meeting of The Satellite Division of the Institute of Navigation, Tampa FL, 2015.

[27] R. L. Freeman, Radio System Design for Telecommunications, Hoboken NJ: WILEY-INTERSCIENCE, 2007, pp. 23-25,599-602.

[28] L. A. Berry, "Wave Hop Theory of Long Distance Propagation of LowFrequency Radio Waves," RADIO SCIENCE Journal of Research NBS/USNCURSI, vol. 68D, no. 12, pp. 1275-1284, 1964.

[29] "Introduction to HF Radio Propagation," Australian Government - Bureau of Meterology, 2016. [Online].

Available: http://www.sws.bom.gov.au/Educational/5/2/2. [Accessed May 2018].

[30] J. A. Bennett, "The Ray Theory of Doppler Frequency Shift," Australian Journal of Physics, no. 21, 1967.

[31] P. E. Gill, W. Murray and M. H. Wright, PRACTICAL OPTIMIZATION, San Diego CA: Academic Press Limited, 1995, pp. 134-137.

[32] J. Nocedal and S. J. Wright, Numerical Optimization, Springer, 2006, pp. 254259.

[33] D. P. Bertsekas, Nonlinear Programming, Belmont MA: Athena Scientific, 1995. 
[34] S. Boyd and V. Lieven, Convex Optimization, Cambridge, UK: University Press, 2004.

[35] P. Gregory, Bayesian Logical Data Analysis for the Physical Sciences, Cambridge UK: Cambridge University Press, 2005.

[36] NASA Administration, "International Reference Ionosphere," [Online]. Available: http://iri.gsfc.nasa.gov/. [Accessed 4 August 2016].

[37] D. Kenneth, "Ionospheric Radio Propagation," National Bureau of Standards , Washington D.C., 1965.

[38] D. L. Hysell, M. Milla and J. Vierinen, "A Multistatic HF Beacon Network for Ionospheric Specification in the Peruvian Sector," Radio Science, vol. 51, no. 5, pp. 392-401, 2016.

[39] M. L. Psiaki and S. Mohiuddin, "Global Positioning System Integer Ambiguity Resolution Using Factorized Least-Squares Techniques," Journal of Guidance, Control, and Dynamics, vol. 30, no. 2, pp. 346-356, 2007.

[40] L. Scherliess, R. W. Schunk, J. J. Sojka, D. C. Thompson and L. Zhu, "Utah State University Global Assimilation of Ionospheric Measurements GaussMarkov Kalman Filter Model of The Ionosphere: Model Description and Validation," vol. 111, 2006. 
APPENDIX A

\section{AUXILIARY CALCULATIONS FOR PHYSICAL AND MATHEMATHICAL MODELS}

\section{A.1 The $\underline{o}$ Function}

An auxiliary three-term-output vector function $\underline{o}$ performs a one-dimensional interpolation of the bivariate function $f(x, w)$ at an arbitrary point $\left(x_{0}, w_{0}\right)$ given boundary conditions at two points $-\left(x_{1}, w_{0}\right)$ and $\left(x_{2}, w_{0}\right)$. The required input arguments for this function includes a set of partial derivatives of $f(x, w)$ with respect $x$ evaluated at $\left(x_{1}, w_{0}\right)$ and at $\left(x_{2}, w_{0}\right)$. In the context of this study, $y$ is used with bi-quintic splines computations. It takes the general form:

$$
\begin{aligned}
& {\left[f\left(x_{0}, w_{0}\right)\right.}\left.\frac{\partial f}{\partial x}\left(x_{0}, w_{0}\right) \quad \frac{\partial^{2} f}{\partial x^{2}}\left(x_{0}, w_{0}\right)\right]= \\
& \underline{O}\left(x_{1}, f\left(x_{1}, w_{0}\right), \frac{\partial f}{\partial x}\left(x_{1}, w_{0}\right), \frac{\partial^{2} f}{\partial x^{2}}\left(x_{1}, w_{0}\right), x_{2}, f\left(x_{2}, w_{0}\right)\right. \\
&\left., \frac{\partial f}{\partial x}\left(x_{2}, w_{0}\right), \frac{\partial^{2} f}{\partial x^{2}}\left(x_{2}, w_{0}\right), x_{0}\right)
\end{aligned}
$$

The three-term output of $y$ is computed as follows. Define:

$$
\Delta x=x_{2}-x_{1}
$$

and compute 


$$
\begin{aligned}
b_{0}= & f\left(x_{1}, w_{0}\right) \\
b_{1}= & \frac{\partial f}{\partial x}\left(x_{1}, w_{0}\right) \\
b_{2}= & 0.5 \frac{\partial^{2} f}{\partial x^{2}}\left(x_{1}, w_{0}\right) \\
b_{3}= & \left(-10 f\left(x_{1}, w_{0}\right)+10 f\left(x_{2}, w_{0}\right)-6 \frac{\partial f}{\partial x}\left(x_{1}, w_{0}\right) \Delta x-4 \frac{\partial f}{\partial x}\left(x_{2}, w_{0}\right) \Delta x\right. \\
& \left.-1.5 \frac{\partial^{2} f}{\partial x^{2}}\left(x_{1}, w_{0}\right) \Delta x^{2}+0.5 \frac{\partial^{2} f}{\partial x^{2}}\left(x_{2}, w_{0}\right) \Delta x^{2}\right) \frac{1}{\Delta x^{3}} \\
b_{4}= & \left(15 f\left(x_{1}, w_{0}\right)-15 f\left(x_{2}, w_{0}\right)+8 \frac{\partial f}{\partial x}\left(x_{1}, w_{0}\right) \Delta x+7 \frac{\partial f}{\partial x}\left(x_{2}, w_{0}\right) \Delta x\right. \\
& \left.+1.5 \frac{\partial^{2} f}{\partial x^{2}}\left(x_{1}, w_{0}\right) \Delta x^{2}-\frac{\partial^{2} f}{\partial x^{2}}\left(x_{2}, w_{0}\right) \Delta x^{2}\right) \frac{1}{\Delta x^{4}} \\
b_{5}= & \left(-6 f\left(x_{1}, w_{0}\right)+6 f\left(x_{2}, w_{0}\right)-3 \frac{\partial f}{\partial x}\left(x_{1}, w_{0}\right) \Delta x-3 \frac{\partial f}{\partial x}\left(x_{2}, w_{0}\right) \Delta x\right. \\
& \left.-0.5 \frac{\partial^{2} f}{\partial x^{2}}\left(x_{1}, w_{0}\right) \Delta x^{2}+0.5 \frac{\partial^{2} f}{\partial x^{2}}\left(x_{2}, w_{0}\right) \Delta x^{2}\right) \frac{1}{\Delta x^{5}}
\end{aligned}
$$

Then:

$$
\begin{aligned}
f\left(x_{0}, w_{0}\right)= & b_{0}+b_{1}\left(x_{0}-x_{1}\right)+b_{2}\left(x_{0}-x_{1}\right)^{2}+b_{3}\left(x_{0}-x_{1}\right)^{3} \\
& +b_{4}\left(x_{0}-x_{1}\right)^{4}+b_{5}\left(x_{0}-x_{1}\right)^{5} \\
\frac{\partial f}{\partial x}\left(x_{0}, w_{0}\right)= & b_{1}+2 b_{2}\left(x_{0}-x_{1}\right)+3 b_{3}\left(x_{0}-x_{1}\right)^{2}+4 b_{4}\left(x_{0}-x_{1}\right)^{3} \\
& +5 b_{5}\left(x_{0}-x_{1}\right)^{4} \\
\frac{\partial^{2} f}{\partial x^{2}}\left(x_{0}, w_{0}\right)= & 2 b_{2}+6 b_{3}\left(x_{0}-x_{1}\right)+12 b_{4}\left(x_{0}-x_{1}\right)^{2}+20 b_{5}\left(x_{0}-x_{1}\right)^{3}
\end{aligned}
$$




\section{APPENDIX B \\ TECHNICAL NOTES FOR THE SIMPLIFIED RAY- PATH MODEL FILTER}

\section{B.1 Computing Sensitivity of Bounce-Points Equations to Bounce-Points Location}

With the simplified ray-path model, special care must be taken with the Jacobian

$\partial g / \partial \underline{\eta}$ of the relationship between the bounce-point equation outputs that are set equal to zero and bounce-points' locations.

Since some terms of $\tilde{g}_{\text {simp }}$ contain terms defined in Cartesian ECEF coordinates, geographic LLA coordinates, and in terms of the partial derivatives of the geographic LLA coordinates with respect to the Cartesian ECEF coordinates, it is necessary to deal carefully with some entries of the Jacobian matrix. Additional complexity arises from the manner in which the $\tilde{g}_{\text {simp }}$ functions depend on the unknown $\underline{p}_{j}$ parameters, which are used to compute the three Chapman profile parameters via a bi-quintic latitude/longitude spline.

Let $\underline{e}=\left\{x_{\mathrm{k}}, y_{\mathrm{k}}, z_{\mathrm{k}}\right\}$ be the set of ECEF coordinates representing the $k^{\text {th }}$ bounce-point, and let $\underline{l}=\left\{\phi_{\mathrm{k}}, \lambda_{\mathrm{k}}, a l_{\mathrm{k}}\right\}$ be the equivalent set of this point's LLA coordinates. Then, for a TypeA equation that applies at an ionospheric bounce point $k$, the desired sensitivity $1 \times 3$ vector is given by 


$$
\frac{\partial g_{A, k}}{\partial \underline{e}}=\frac{\partial g_{A, k}}{\partial \underline{l}} \Lambda
$$

where the right most Jacobian, $\Lambda=\partial \underline{\underline{l}} / \partial \underline{\underline{e}}$, is that of the standard Cartesian-to-lat/long/alt transformation for WGS-84 coordinates.

For ionosphere bounce-points' Type-B equations, recall that these equations were derived using the normal vector that applies at an ionosphere reflection point. The vector is defined as the negative gradient of the electron density field with respect to Cartesian ECEF coordinates. The electron density model is modeled using latitude/longitude dependence. Consequently, these equations are defined in terms of both geographic LLA coordinates and their partial derivatives with respect to Cartesian ECEF coordinates. Therefore, when one takes odd numbered Type-B equations' derivatives with respect to a set of Cartesian coordinates, three groups of terms, corresponding with three types of dependencies, should be evaluated. The total derivative of the $k^{\text {th }}$ Type-B equation with respect to the $j^{\text {th }}$ term of $\underline{e}$ takes the form

$$
\begin{aligned}
& \frac{D g_{B, k}}{D e_{j}}= \frac{\partial g_{B, k}}{\partial e_{j}}+\sum_{i=1}^{3} \frac{\partial g_{B, k}}{\partial l_{i}} \Lambda_{i, j}+\sum_{i=1}^{3} \sum_{i=m}^{3} \frac{\partial g_{B, k}}{\partial D_{i, \mathrm{~m}}} \frac{\partial \Lambda_{i, \mathrm{~m}}}{\partial e_{j}} \\
&(\text { i }) \quad \\
&= \frac{\partial g_{B, k}}{\partial e_{j}}+\sum_{i=1}^{3} \frac{\partial g_{B, k}}{\partial l_{i}} \frac{\partial l_{i}}{\partial e_{j}}+\sum_{i=1}^{3} \sum_{m=1}^{3} \frac{\partial g_{B, k}}{\partial\left(\frac{\partial l_{i}}{\partial e_{m}}\right)} \frac{\partial^{2} l_{i}}{\partial e_{m} \partial e_{j}} \\
&\left(i i i_{b}\right)
\end{aligned}
$$

Computation of term (i) is immediate. The single-index summation (ii) includes the partial derivatives of the equation with respect to geographic LLA coordinates and terms 
of $\Lambda$. The double-indices summation (iii) considers the partial derivatives of the equations with respect $\Lambda$ - term $\left(i i i_{a}\right)$. These terms are multiplied by terms of the ECIF to LLA coordinates-conversion Hessian (iiib). This unconventional formulation is needed since the entries of $\Lambda$ cannot be represented analytically in an explicit form and therefore cannot be differentiated. Instead they are known numerically, together with their partial derivatives with respect to the desired quantities of $\underline{\underline{e}}$. Finally, it should be recognized that Eq. (130) applies for Type-C equations as well.

\section{B.2 Setting up the $\partial g / \partial \eta$ Sensitivity Matrix}

The procedure of populating the entries of the Jacobian $\partial g / \partial \eta$ that was discussed in the previous subsection is technical, although somewhat complicated, with a variety of different types of terms. Computations for the various terms depend on equation type, bounce-point index, and the total number of bounce-points in the considered ray-path. This subsection briefly lists these subcases, yet it does not provide the explicit formulas that require additional derivation which goes beyond the scope of this text.

As an example, consider a ray-path with four bounce points. The structure of its $12 \times 12$ Jacobian sensitivity matrix is illustrated in Fig. 78, where non-zero terms are highlighted in gray. Matrix rows correspond with Type- $\mathrm{A} / \mathrm{B} / \mathrm{C}$ equations and matrix columns correspond with ECEF $\mathrm{x} / \mathrm{y} / \mathrm{z}$ coordinates, as labelled in the figure. The 1-4 numbering corresponds to the four bounce points. 


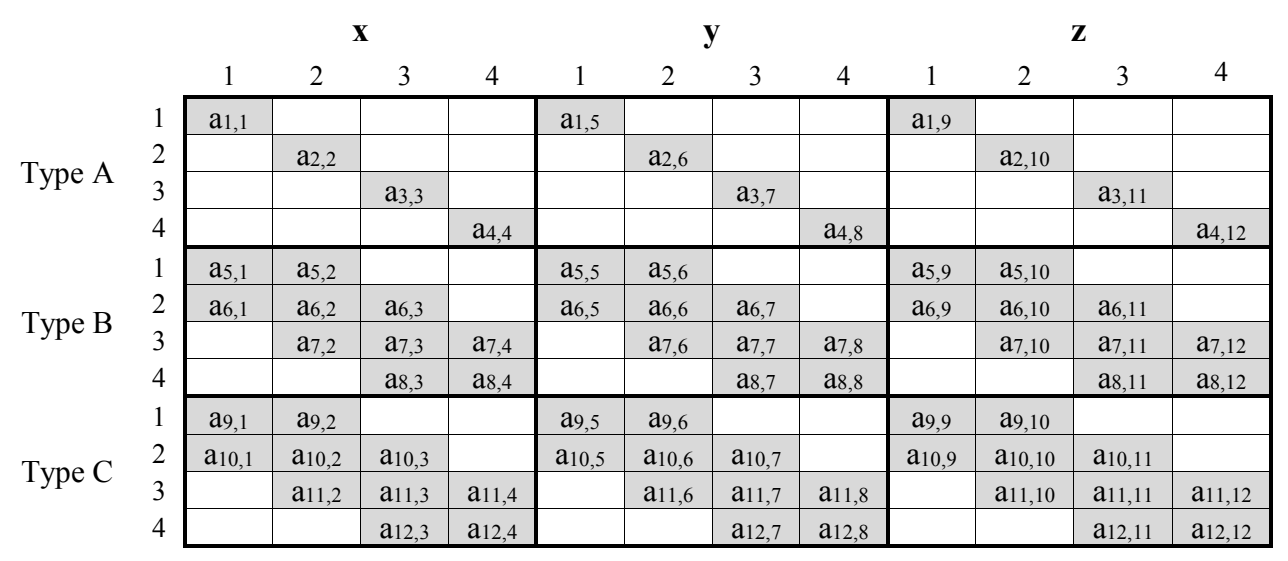

Figure 78: The form of a 4 bounce-points ray-path's Jacobian sensitivity matrix.

For Type-A equations, $a_{n, n}$ terms that relate $g$ equations with the $x / y / z$ coordinates of the four bounce-points have a certain form for odd $n$ and a different form for even $n$. this is a consequence of the former defined with Cartesian coordinates and the latter defined with geographic coordinates. The same holds for $a_{n, n+m}$ and $a_{n, n+2 m}$ terms where $m$ is the number of bounce points.

For Type B equations, row 5 (or $m+1$ ) has a certain form, where only six entries are non-zero and the top left entries of each sub-block (i.e. $a_{5,1}, a_{5,5}$ and $a_{5,9}$ ) include coordinate terms of the transmitter's position. Other rows related to Type B equations, except for the last row (row 8 (or $2 m$ ) of the whole matrix), have nine non-zero entries and their forms depend on whether their index is odd or even. Additionally, these rows do not consider the location of either the transmitter or the receiver. The last row $(2 m)$ has six non-zero terms, where the bottom right entry of each sub-block (i.e. $a_{8,4}, a_{8,8}$ and $a_{8,12}$ ) include terms of the position of the receiver. In this example with an even number of bounce points, this row will be utilizing Cartesian coordinates since the index of the last row is four (row eight of the whole matrix). Had there been a fifth bounce-point, these 
terms would have had a different form that originates from the geographic coordinates used with ionosphere bounce-points.

Type-C equations are similar to Type-B equations in terms of their structure, and thus the same observations which were made for Type-B related sub-blocks apply.

In summary, when setting up this Jacobian matrix, twelve subcases of the matrix's entries forms should be considered:

1. Type-A equations (two subcases)

- Rows with odd index

- Rows with even index

2. Type-B equations (five subcases)

- First row

- Rows whose odd index is greater than one and less than the number of bounce points

- Row whose even index is greater than one and less than the number of bounce points

- Last row with odd index

- Last row with even index

3. Type-C equations (five subcases) - same as with Type-B equations 


\section{APPENDIX C}

\section{IMPLEMENTATION AND PRACTICALITIES}

MATLAB $^{\circledR}$ CODE is used with this study to construct the different components of the truth-model simulation and the batch nonlinear least-squares filter.

\section{C.1 Software Concepts}

- The code is characterized with a clear distinction between different software component types, as described in the next subsection.

- Intensive use of MATLAB ${ }^{\circledR}$ symbolic toolbox for generating objects that are used to obtain needed terms and formulas using embedded symbolic mathematical operators. This design in meant to provide flexibility when changes to physical models are to be performed.

- Flexibility in combining and connecting components from different sources: code generated for different phases of this study, code provided by Prof. Mark Psiaki, and code that was obtained from various websites (free use license only).

- Use of code that is generated automatically by auxiliary MATLAB ${ }^{\circledR}$ functions in order to minimize potential coding errors.

- The use of an auto-testing code array to validate functionality of functions and algorithms. 


\section{C.2 Code components}

The code used is fundamentally split into five groups of components.

- Functions that contain batch-estimation code that would potentially run on a receiver are considered Real Time Code (though, not 'real time' in its formal meaning as these modules' run time is usually hard to predict). Real time code can be generated either manually or automatically, i.e., generated by other functions.

- Simulation Code, including scripts, functions and GUIDE ${ }^{\circledR}$ objects that are used to generate the simulation environment, compute truth-model data, run the batchfiltering algorithm by calling Real Time Code, monitor its progress and to present simulation output results.

- Derivation Code. This set of scripts and functions is used to generate additional code, including: (a) Construction of symbolic MATLAB ${ }^{\circledR}$ objects (b) derivation of needed objects such as equations, formulas and Jacobians, and (c) Automatic generation of code using the derived symbolic objects.

- Core Code. Generic functions that are used by different types of functions. Raytracing code, for instance, falls under this category as it is used by both truthmodel real time code and the simulation code.

- Test Code. These scripts are used in testing functions and code segments, including finite differencing tests. 


\section{C.3 Hardware and Process Runtime}

The extensive computations that are involved in the solution process were mostly carried out on an Intel ${ }^{\circledR}$ Xeon ${ }^{\circledR}$ model E7-8867-V4 based workstation with four central processing units and a total of 72 cores.

Typical runtime for a single ray-path solving procedure is 30 seconds to 4 minutes. Typical runtime for a single test case of the batch nonlinear least-square filter, excluding truth-model calculations, is in the order of hours. The author conjectures that, with the combination of (a) a dedicated hardware, (b) more efficient process parallelization, and (c) use of low-level coding language, batch filter execution times could be reduced to an order of a few minutes. ${ }^{3}$

${ }^{3}$ I thank Cornell University Prof. Dmitry Savransky for exceptional technical advice on both software and hardware. 